\title{
A Variable Stability In-Flight Simulation System using Incremental Non-Linear Dynamic Inversion
}

MSc. Thesis

P.A. Scholten

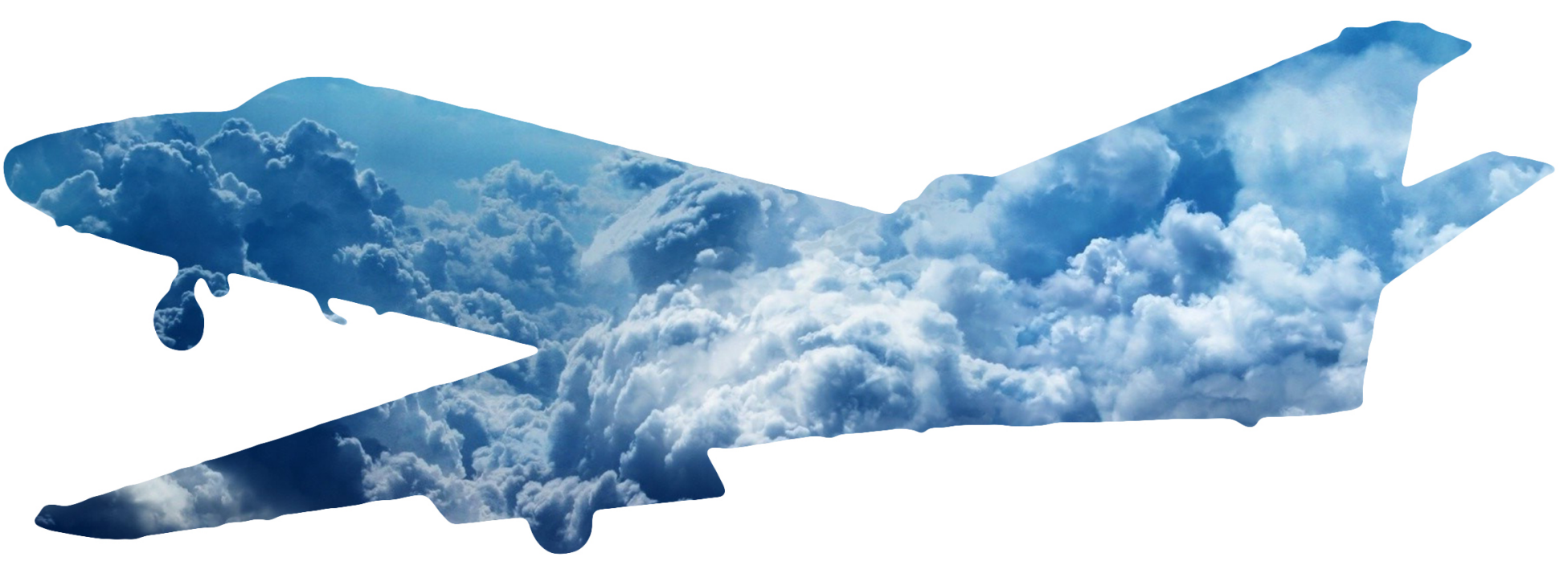





\title{
A Variable Stability In-Flight Simulation System using Incremental Non-Linear Dynamic Inversion
}

\author{
MSc. Thesis
}

by

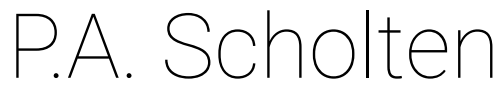

to obtain the degree of Master of Science

at the Delft University of Technology,

Student number:

Project duration:

Thesis committee:
4214099

April 4, 2018 - January 24, 2019

Dr. Ir. M.M. (René) van Paassen, TU Delft, First supervisor

Prof. Dr. Ir. M. (Max) Mulder, TU Delft, supervisor

Dr. Ir. J.C.F. (Joost) de Winter, $\quad$ TU Delft, external examiner

An electronic version of this thesis will be made available to the TU Delft repository at http://repository.tudelft.nl/. 



\section{List of Figures}

1.1 Research framework for the thesis project . . . . . . . . . . . . . . . . . . . . 24

1.2 The structure of the proposed thesis research methodology . . . . . . . . . . . . . . . 25

2.1 CAL variable stability system development aircraft $[2] \ldots \ldots \ldots \ldots \ldots$. . . . . . . . . . 28

2.2 Lockheed NT-33A variable stability system development [2] . . . . . . . . . . . . . . . . . . 28

2.3 Convair NC-131H TIFS variable stability system development [2] . . . . . . . . . . . . . . . . 29

2.4 CAL modern variable stability systems $[2] \ldots \ldots \ldots \ldots \ldots \ldots \ldots \ldots$

2.5 Grumman C-11A STA variable stability aircraft $[2] \ldots \ldots \ldots \ldots \ldots \ldots \ldots$

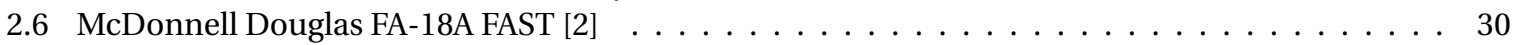

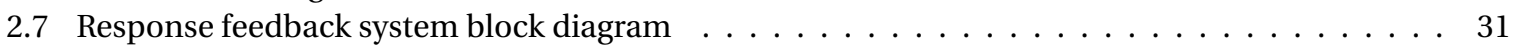

2.8 Explicit model-following command generator tracker for $d=3 \ldots \ldots \ldots \ldots \ldots$

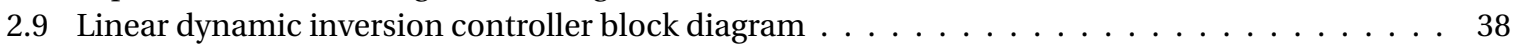

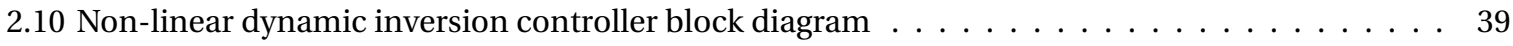

2.11 Simplified non-linear dynamic inversion controller block diagram . . . . . . . . . . . . . . 40

2.12 CAP short period requirements for Class II, Category B aircraft . . . . . . . . . . . . . . . . . 43

2.13 Cooper-Harper rating scale decision tree $[4] \ldots \ldots \ldots \ldots \ldots \ldots \ldots$

2.14 General manoeuvre characteristics $[32] \ldots \ldots \ldots \ldots \ldots \ldots \ldots \ldots$

2.15 Design parameters related to manoeuvres $[32] \ldots \ldots \ldots \ldots \ldots \ldots \ldots$

3.1 Schematic overview of the non-linear actuator model $\ldots \ldots \ldots \ldots \ldots$

3.2 Schematic overview of the non-linear elevator model $[35] \ldots \ldots \ldots \ldots$. . . . . . . . . . 52

3.3 Comparison elevator model and actual response from flight test . . . . . . . . . . . . . 53

3.4 Comparison elevator model and actual response from flight test with updated hinge moment . 53

3.5 Comparison elevator model and actual response from flight test with updated hinge moment and feedforward gain . . . . . . . . . . . . . . . . . . . . . 54

3.6 Comparison elevator model and actual response from flight test with updated hinge moment, feedforward gain and corrected limit detection . . . . . . . . . . . . . . . . 55

3.7 Comparison corrected elevator model and actual response from flight test for different flight

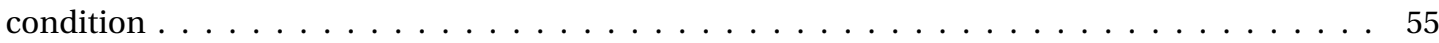

3.8 Comparison aileron model and actual response from flight test . . . . . . . . . . . . . . 56

3.9 Comparison aileron model and actual response from flight test with updated feedforward gain . 56

3.10 Comparison corrected aileron model and actual response from flight test for servo current sat-

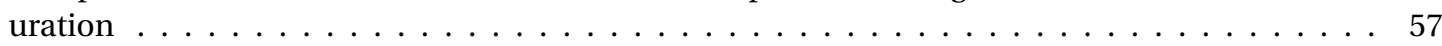

3.11 Comparison corrected aileron model and actual response from flight test for different flight

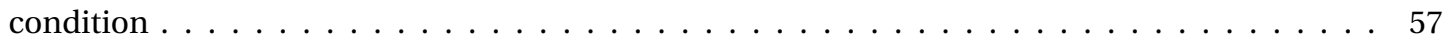

4.1 Schematic overview of the CitAST model for the Cessna Citation $500 \ldots \ldots \ldots$

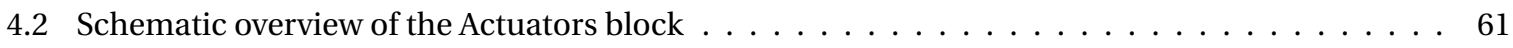

4.3 Modified schematic overview of the CitAST model for the Cessna Citation 500 . . . . . . . . . 61

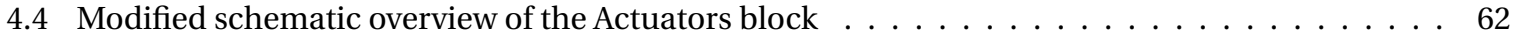

4.5 Schematic overview of the iterative trim procedure . . . . . . . . . . . . . . 63

4.6 Multiplier parameter from CitAST model as a function of angle of attack and Mach number . . . 64

4.7 Multiplier parameter from CitAST model as a function of angle of attack and flap deflection . . 64

4.8 Second multiplier parameter from CitAST model as a function of angle of attack and flap de-

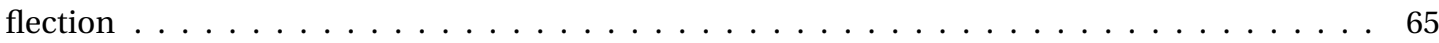

4.9 Multiplier parameter from CitAST model as a function of indicated airspeed . . . . . . . . . 65

4.10 Longitudinal moment effectiveness compared for different initial conditions . . . . . . . . 66

4.11 Maximum elevator deflection compared for different initial conditions . . . . . . . . . . . . 66

4.12 Variable stability system limitations in flight envelope . . . . . . . . . . . . . . . . 67 
5.1 Controlled rate of climb $(+/-6 \mathrm{~m} / \mathrm{s})$ with combined actuator and aircraft model $\ldots \ldots$. . . . . 69

5.2 Controlled rate of climb $(+12$ and $-10 \mathrm{~m} / \mathrm{s})$ with combined actuator and aircraft model . . . . 70

5.3 Equation Error and Output Error estimation . . . . . . . . . . . . . . . . . 71

5.4 CAP estimation of the Cessna Citation 500 at different initial conditions $\ldots \ldots \ldots \ldots$. . . . 71

B.1 Schematic block diagram of the controller structure $\ldots \ldots \ldots \ldots \ldots \ldots$

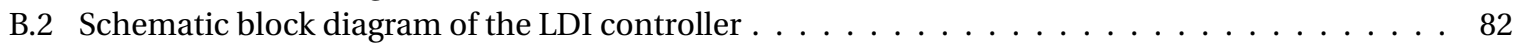

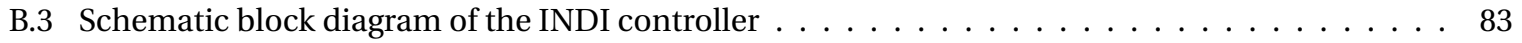

B.4 Modified schematic overview of the CitAST model for the Cessna Citation 500 . . . . . . . . . . . 84

B.5 Schematic overview of the modified basic actuator block . . . . . . . . . . . . . . . 84

B.6 Modified schematic overview of the Actuators block . . . . . . . . . . . . . . . . . . 84

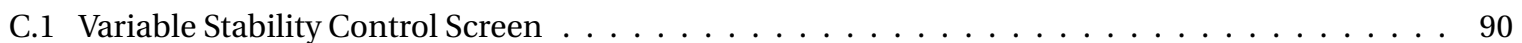

C.2 Fly-by-wire system control screen $\ldots \ldots \ldots \ldots \ldots \ldots \ldots \ldots \ldots$

D.1 Aircraft pitch performance to step command on pitch rate . . . . . . . . . . . . . . 92

D.2 Aircraft roll performance to step command on roll rate . . . . . . . . . . . . . . . . . . . . 92

D.3 Aircraft pitch response to 45 degrees 16 kts wind disturbance . . . . . . . . . . . . . . . . . . . 94

D.4 Aircraft roll response to 45 degrees $16 \mathrm{kts}$ wind disturbance . . . . . . . . . . . . . . . . . . . . 94

D.5 Aircraft pitch response for different CAP and damping reference models, INDI controller with

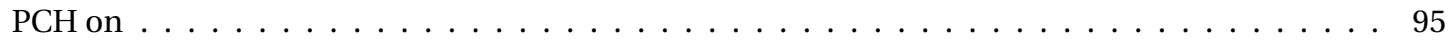

D.6 Aircraft pitch response for different CAP 1.1 and damping 0.3 with the approximate actuator

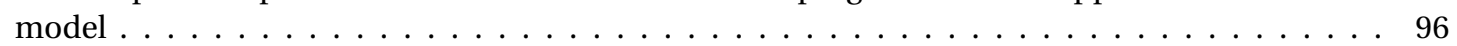

D.7 Aircraft pitch response for different CAP 1.1 and damping 0.3 with the actuator model from Lubbers $[35] \ldots \ldots \ldots \ldots \ldots \ldots \ldots$

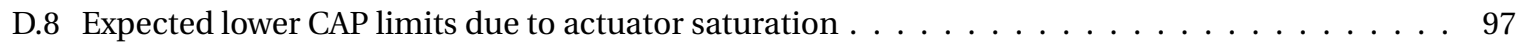

E.1 Vertical Speed Indicator flight display . . . . . . . . . . . . . . . . . . . . . . . . 100

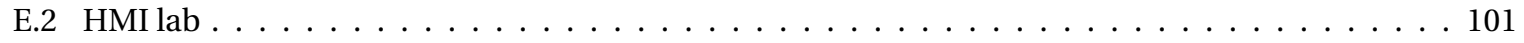

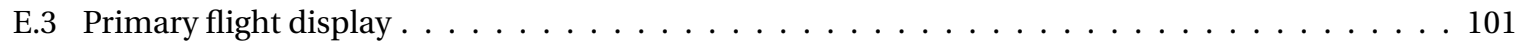

E.4 Engine display . . . . . . . . . . . . . . . . . . . . . . . . . . . . . . . . 102

E.5 Flow diagram concerning the independent variable with two conditions . . . . . . . . . . . . 102

E.6 Initial CAP and Damping conditions set in the CAP reference model . . . . . . . . . . . . . . 103

E.7 Experiment matrix showing different test conditions per run per subject . . . . . . . . . . . . . 104

E.8 Means and the 95\% confidence limits of the combined flag sorted by CAP and Damping ratio . . 116

E.9 Means and the 95\% confidence limits of the control activity sorted by model and Cooper-Harper difference $(\mathrm{C}=\mathrm{CAP}$ reference model $; \mathrm{F}=$ Full model $) \ldots \ldots \ldots \ldots$

E.10 Means and the $95 \%$ confidence limits of the task performance sorted by model and CooperHarper difference $(\mathrm{C}=\mathrm{CAP}$ reference model $\mathrm{F}=$ Full model $) \ldots \ldots \ldots$. . . . . . . . . . 117

E.11 Means and the $95 \%$ confidence limits of the combined flag with counted flag duration larger than 0.2 seconds $\ldots \ldots \ldots \ldots \ldots \ldots \ldots \ldots \ldots \ldots \ldots$

F.1 Cessna Citation II laboratory aircraft with boom configuration . . . . . . . . . . . . . . . . . 120

F.3 Comparison of IMAR IMU accelerations and angular accelerometer accelerations . . . . . . . 122

F.2 Oscillatory response of aircraft without additional pilot input . . . . . . . . . . . . . . . 122 


\section{List of Tables}

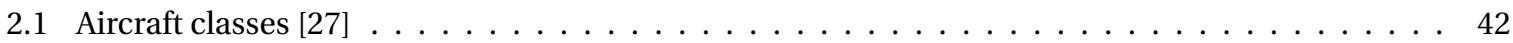

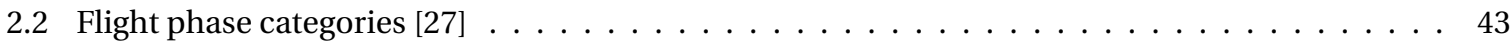

2.3 Levels of acceptability $[27] \ldots \ldots \ldots \ldots \ldots \ldots \ldots \ldots \ldots \ldots \ldots \ldots \ldots$

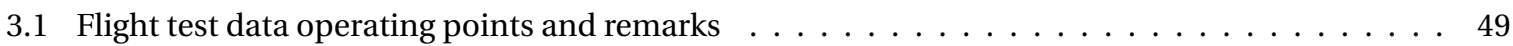

3.2 Actuator model inputs Lubbers model . . . . . . . . . . . . . . . . . . . . . . 50

4.1 Description of pilot inputs to the CitAST model $\ldots \ldots \ldots \ldots \ldots \ldots$

5.1 Maximum rate of climb due to elevator deflection limitations . . . . . . . . . . . . . . . 70

A.1 Variable stability limitations for different initial flight conditions . . . . . . . . . . . . . . 79

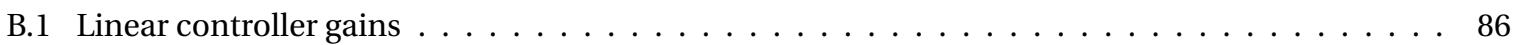

E.1 Model independent experimental condition . . . . . . . . . . . . . . . . . . . . 102

E.2 Characteristics of the pilot subjects $\ldots \ldots \ldots \ldots \ldots \ldots$

F.1 Sensor characteristics of the Cessna Citation II Laboratory Aircraft [40] [41] [36] . . . . . . . . 121

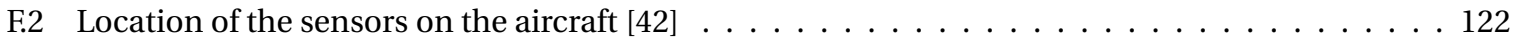





\section{List of Abbreviations}

CAL Calspan Corporation. 27, 28, 29, 73

CAP Control Anticipation Parameter. 23, 24, 32, 42, 43, 44, 45, 69, 71, 74, 81, 85, 87, 90, 91, 95, 96, 116

DASMAT Delft University Aircraft Simulation Model and Analysis Tool. 48, 61, 82, 83, 87, 88, 119

DLR Deutsches Zentrum für Luft- und Raumfahrt. 25

DUECA Delft University Environment for Communication and Activation. 48, 50, 81, 87, 88, 90, 120

FAST Full-scale Advanced System Testbed. 30, 39

GUI Graphical User Interface. 88

HEFTIG Highly Effective Flight Test Instrumentation GUI. 49, 50

HMI Human Machine Interaction. 88

HOS High Order System. 42, 44, 71, 74

IMU Inertial Measurement Unit. 82, 120, 122

INDI Incremental Non-linear Dynamic Inversion. 37, 39, 40, 41, 81, 82, 83, 85, 88, 91, 93, 119

LDI Linear Dynamic Inversion. 81, 82, 91, 93

LOES Low Order Equivalent System. 42, 44, 71, 74

LQ Linear Quadratic. 32

MAV Micro Aerial Vehicle. 41

NACA National Advisory Committee for Aeronautics. 27

NASA National Aeronautics and Space Administration. 27, 29, 30, 39

PCH Pseudo-Control Hedging. 81, 84, 85, 87, 90, 91, 93, 95, 96

PI Performance Index. 32, 33, 34, 35

PID Proportional Integral Derivative. 41

PINDI Predictive Incremental Non-linear Dynamic Inversion. 40, 41

STA Shuttle Training Aircraft. 30

TIFS Total In-Flight Simulator. 28

UAV Unmanned Aerial Vehicle. 29

US United States. 27, 28, 29

VISTA Variable Stability In-Flight Simulator Test Aircraft. 29 



\section{List of Symbols}

$\dot{x}$ State vector derivative. 31

$\xi$ Modified state. 35

$\mu$ Modified control input. 35

$\theta$ Pitch angle $[\mathrm{rad}] .42$

$\tau$ Time delay $[s] .44$

$\rho$ Air density $\left[\mathrm{kg} / \mathrm{m}^{3}\right] .63,82$

$\alpha$ Angle of attack [ $\mathrm{rad}] .63$

$\delta_{I F S}$ Baseline flight control actuator command. 38

$\delta_{V S S}$ Variable stability system actuator command. 38

$\alpha_{f}$ Fading factor. 38

$\delta_{\text {flaps }}$ Deflection of the flaps [ $\left.\mathrm{rad}\right] .63$

A State matrix. 31

$B$ Input matrix. 31

$C_{m_{\delta_{e}}}$ Moment coefficient due to elevator deflection [-].63

$I_{y}$ Moment of Inertia around the y-axis $\left[\mathrm{kg} \mathrm{m}^{2}\right] .59$

$J$ Cost function value. 44

$J$ Inertia tensor. 83

$K$ Gain matrix. 31

$M$ Moment $[N m] .59$

$M$ Mach number [-]. 63

$M_{\delta_{e}}$ Moment due to elevator deflection [Nm]. 59

$M_{A}$ Aerodynamic moment $[\mathrm{Nm}] .82$

$M_{T}$ Moment induced by thrust $[\mathrm{Nm}] .82$

$M_{a}$ Airframe moment [Nm]. 82

$M_{c}$ Control effectors moment $[\mathrm{Nm}] .82$

$Q$ Weighting matrix. 32

$R$ Weighting matrix. 32

$S$ Ricatti matrix. 32

$S$ Wing surface area $\left[\mathrm{m}^{2}\right] .63,82$

$V$ Velocity $[\mathrm{m} / \mathrm{s}] .42$ 
$V_{\text {ias }}$ Indicated airspeed $[\mathrm{m} / \mathrm{s}] .63$

$V_{\text {tas }}$ True airspeed $\left[\mathrm{m} / \mathrm{s}^{2}\right] .63,82$

$a_{i}$ Degree. 35, 36

$b$ Span $[m] .82$

$c$ Chord length $[m] .63,82$

$d$ Degree. 35

g Gravitational acceleration $\left[\mathrm{m} / \mathrm{s}^{2}\right] .42$

$n_{z}$ Normal acceleration $\left[\mathrm{m} / \mathrm{s}^{2}\right] .42$

$q$ Pitch rate $[\mathrm{rad} / \mathrm{s}] .42$

$r$ Reference command. 35, 36, 37

$t$ Time $[s] .31$

$u$ Control input. 37, 39, 59

$u_{e}$ Input error. 31

$u_{f}$ Input feedback. 31

$u_{p}$ Input pilot. 31

$v$ Auxiliary input. 37, 38, 39

$x$ State vector. $31,33,37,39$

y Output. 37, 38, 39

$z$ Performance output. 34 


\section{Contents}

List of Figures $\quad$ iii

List of Tables $\quad v$

List of Abbreviations $\quad$ vii

List of Symbols $\quad x$

I Scientific Paper 1

II Preliminary Thesis

Previously graded under AE4020 21

1 Introduction 23

1.1 Research objective . . . . . . . . . . . . . . . . . . . . . . . . . . . . . . . 24

1.2 Research question . . . . . . . . . . . . . . . . . . . . . . . . . . 24

1.3 Methodology and report structure . . . . . . . . . . . . . . . . . . . . . . 25

2 Literature study $\quad 27$

2.1 Historical overview . . . . . . . . . . . . . . . . . . . . . . . . . 27

2.1.1 Calspan development . . . . . . . . . . . . . . . . . . . . 27

2.1.2 NASA development . . . . . . . . . . . . . . . . . . . . . . . 29

2.2 Variable stability methods . . . . . . . . . . . . . . . . . . . . . 31

2.2 .1 Response feedback. . . . . . . . . . . . . . . . . . . . . . . 31

2.2 .2 Model-following . . . . . . . . . . . . . . . . . . . . . . . . 33

2.2 .3 Dynamic inversion. . . . . . . . . . . . . . . . . . . . . 37

2.3 Handling Qualities . . . . . . . . . . . . . . . . . . . . . . . . . . 442

2.3.1 Control anticipation parameter . . . . . . . . . . . . . . . . . . . 4 42

2.3.2 High order to low order equivalent system . . . . . . . . . . . . . . . . . 43

2.3.3 Cooper-Harper rating scale . . . . . . . . . . . . . . . . . . . . . . . . . 45

2.3.4 Pilot manoeuvres . . . . . . . . . . . . . . . . . . . . . . . . . . 46

2.4 Simulation models . . . . . . . . . . . . . . . . . . . . . . . . . . . . . . 48

2.4.1 Cessna Citation II aircraft simulation model . . . . . . . . . . . . . . . . . . . . . . . 48

2.4 .2 Actuator model . . . . . . . . . . . . . . . . . . . . . . 48

3 Actuator model validation $\quad 49$

3.1 Flight test data . . . . . . . . . . . . . . . . . . . . . . . . 49

3.2 Model overview . . . . . . . . . . . . . . . . . . . . . . . . . . . . 51

3.3 Elevator. . . . . . . . . . . . . . . . . . . . . . . 53

3.4 Aileron ................................. 56

4 Variable stability limits $\quad 59$

4.1 Methodology ... . . . . . . . . . . . . . . . . . . 59

4.2 Model integration and trimming . . . . . . . . . . . . . . . . . . . . 6 60

4.3 Dynamic inversion limits . . . . . . . . . . . . . . . . . . . . . 6 63

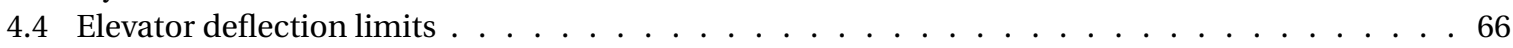

4.5 Operating points in flight envelope . . . . . . . . . . . . . . . . . . . . 67

5 Initial application $\quad 69$

5.1 Rate of climb check . . . . . . . . . . . . . . . . . . . . . . . . . . . . 69

5.2 CAP determination . . . . . . . . . . . . . . . . . . . . 71

6 Conclusion and discussion $\quad 73$ 
$\begin{array}{ll}\text { III Appendices } & 77\end{array}$

A Table of variable stability limitations $\quad 79$

B Controller design $\quad \mathbf{8 1}$

B.1 Inner loop design . . . . . . . . . . . . . . . . . . . . . . . . . . . . 82

B.1.1 LDI logic . . . . . . . . . . . . . . . . . . . . . . 82

B.1.2 INDI logic . . . . . . . . . . . . . . . . . . . . . . . . . . . . . . . . . . . . . . .

B.1.3 System dynamics . . . . . . . . . . . . . . . . . . . . . . . . . . . . . . . . . . . . . . . . . . . . . . . . . . . . .

B.2 Outer loop design . . . . . . . . . . . . . . . . . . . . . . . . . . 85

B.2.1 Sideslip Controller . . . . . . . . . . . . . . . . . . . . . . 85

B.2.2 Pseudo-Control Hedging. . . . . . . . . . . . . . . . . . . . . . 85

B.2.3 CAP Reference Logic . . . . . . . . . . . . . . . . . . . . . . . . . . 85

B.2.4 Sidestick. . . . . . . . . . . . . . . . . . . . . . . 86

B.2.5 Linear Controller. . . . . . . . . . . . . . . . . . . . . . . . . . 86

$\begin{array}{lr}\text { C Software } & \mathbf{8 7}\end{array}$

C.1 MATLAB/Simulink . . . . . . . . . . . . . . . . . . . . . . . . 87

C.2 DUECA software . . . . . . . . . . . . . . . . . . . . . . . 88

C.2.1 Platforms . . . . . . . . . . . . . . . . . . . . . 88

C.2.2 Schematic overview of the DUECA project. . . . . . . . . . . . . . . . . 88

C.2.3 How to control the software? . . . . . . . . . . . . . . . . . . . . . . . 90

D Offline simulations $\quad 91$

D.1 Performance . . . . . . . . . . . . . . . . . . . . . . 91

D.1.1 Pitch . . . . . . . . . . . . . . . . . . . . 91

D.1.2 Roll ................................ 91

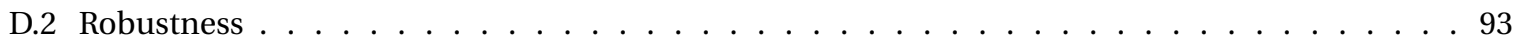

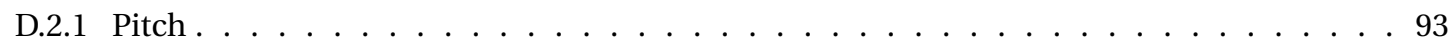

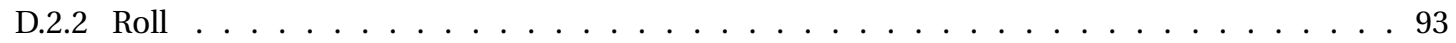

D.3 CAP and Damping simulation. . . . . . . . . . . . . . . . . . . . . 95

D.4 Pseudo Control Hedging . . . . . . . . . . . . . . . . . . . . . . 96

D.5 CAP limits estimation . . . . . . . . . . . . . . . . . . . . . . . . . 97

E Experiment I: Fixed-base simulator $\quad 99$

E.1 Research Problem and Research Question . . . . . . . . . . . . . . . . . . . . . . 99

E.2 Experiment Plan . . . . . . . . . . . . . . . . . . . . . . . 100

E.2.1 Control variables. . . . . . . . . . . . . . . . . . . . . . . . . . 100

E.2.2 Independent variables . . . . . . . . . . . . . . . . . . . . . . . . 102

E.2.3 Dependent variables . . . . . . . . . . . . . . . . . . . . . 103

E.2.4 Initial conditions. . . . . . . . . . . . . . . . . . . . . . . . 103

E.2.5 Experiment matrix and Participants . . . . . . . . . . . . . . . . . . . . . . . 104

E.2.6 Experimental procedures . . . . . . . . . . . . . . . . . . . . . . . . . . . . 104

E.2.7 Hypotheses . . . . . . . . . . . . . . . . . . . . . . . 105

E.3 Experiment instruction manual. . . . . . . . . . . . . . . . . . . . . . . 105

E.4 Experiment script. . . . . . . . . . . . . . . . . . . . . . . . . . . 113

E.5 Additional results experiment. . . . . . . . . . . . . . . . . . . . . . . . . 114

E.5.1 Post-experiment questionnaire . . . . . . . . . . . . . . . . . . . . 114

E.5.2 Additional plots . . . . . . . . . . . . . . . . . . . . . . 116

F Experiment II: Flight test $\quad 119$

F.1 Changes to the controller . . . . . . . . . . . . . . . . . . . . . . . . 119

F.2 Validation plan . . . . . . . . . . . . . . . . . . . . . . 120

F.2.1 Apparatus . . . . . . . . . . . . . . . . . . . . . . 120

F.2.2 Procedure . . . . . . . . . . . . . . . . . . . . . . . 120

F.2.3 Subjects and Instruction to Subjects . . . . . . . . . . . . . . . . . . . . . . 120

F.2.4 Testing report . . . . . . . . . . . . . . . . . . . . . . . . . . . . . 121

F.3 PH-LAB sensor systems . . . . . . . . . . . . . . . . . . . . . . . . 121

F.4 Additional results . . . . . . . . . . . . . . . . . . . . . . . . . . . . . . . . . 122 
Bibliography 



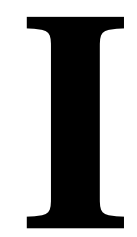

Scientific Paper 



\title{
A Variable Stability In-Flight Simulation System using Incremental Non-Linear Dynamic Inversion
}

\author{
Author: P.A.Scholten*; Supervisors: M.M. van Paassen ${ }^{\dagger}$ and M. Mulder ${ }^{\dagger}$ \\ Delft University of Technology, 2629 Delft, The Netherlands
}

\begin{abstract}
A variable stability in-flight simulator is a system which has the capabilities to change the response of an aircraft in-flight, often without changing the physical properties of the aircraft. The response characteristics and handling qualities can therefore be adjusted, which can be used for many different purposes like pilot training, control logic development and handling quality research. A variable stability control system is designed for a medium range business jet using Incremental Non-linear Dynamic Inversion. The performance of the in-flight simulator is verified by two experiments, one conducted in a fixed-base flight simulator and one in a Cessna Citation II business jet. The fly-by-wire actuation system in the Cessna Citation II is based on its existing autopilot, inheriting the limited performance and safety considerations. The simulator experiment shows differences between the experienced handling qualities for a reference model and the designed controller combined with aircraft dynamics. These differences mainly arise due to actuator saturation for specific handling quality settings. The experiment in-flight supports the performance of the controller in the simulator but also reveals that the available envelope around the initial condition is quite limited due to the constraints of the fly-by-wire system.
\end{abstract}

\section{Nomenclature}

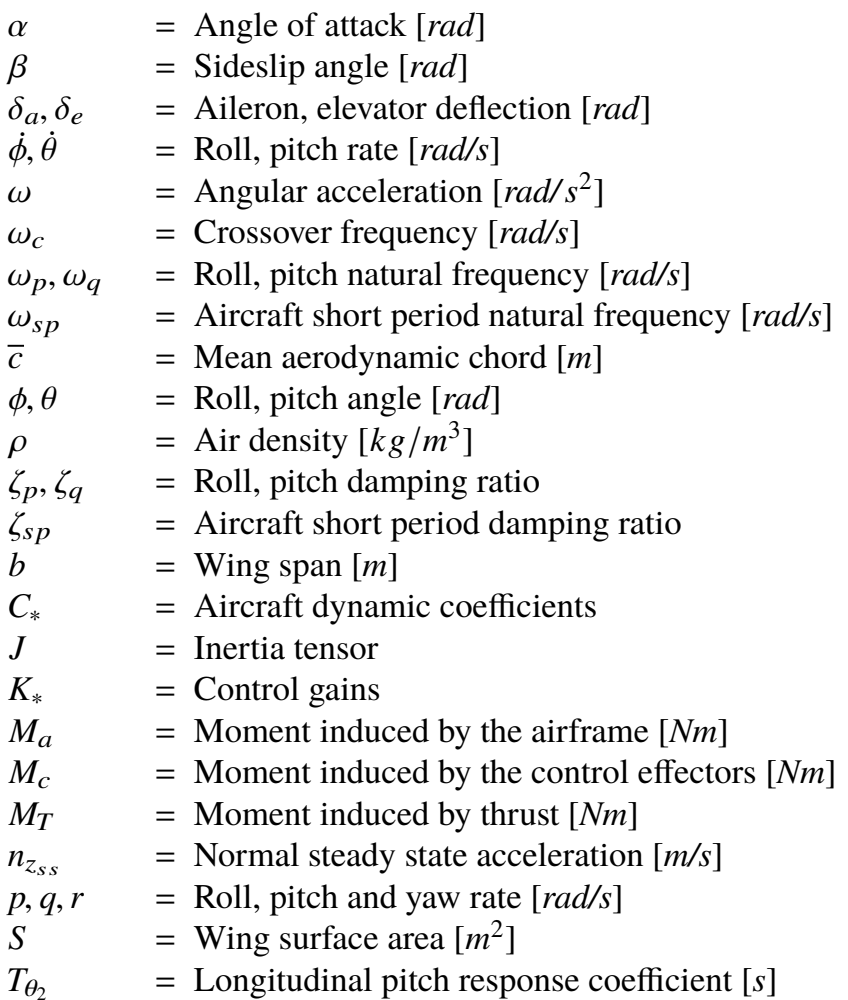

${ }^{*}$ MSc Student, Control and Simulation, Faculty of Aerospace Engineering, Kluyverweg 1; p.a.scholten@live.nl

${ }^{\dagger}$ Associate Professor, Control and Simulation, Faculty of Aerospace Engineering, Kluyverweg 1; m.m.vanpaassen@tudeft.nl. Member AIAA

$¥$ Professor, Control and Simulation, Faculty of Aerospace Engineering, Kluyverweg 1; m.mulder@tudelft.nl. Associate Fellow AIAA

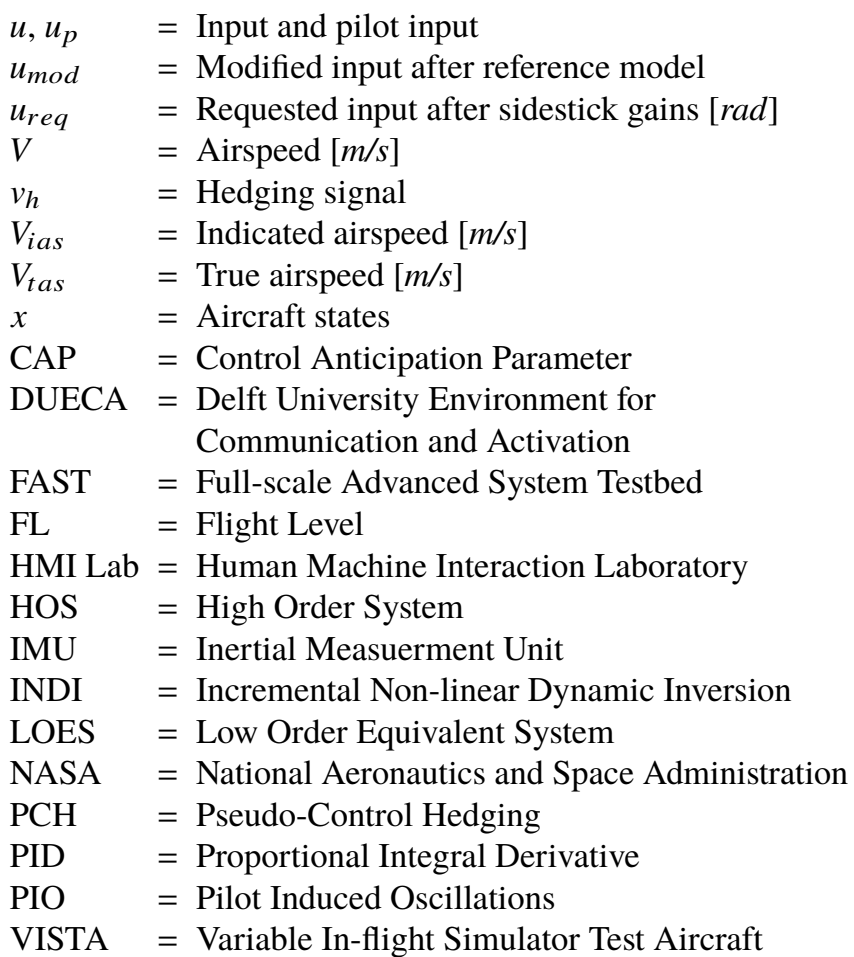

\section{Introduction}

Training of test pilots requires them to experience different handling qualities. It would require different aircraft to provide these different handling qualities, which increases the cost of pilot training. A variable stability in-flight simulator is a system which has the capabilities to change the response of an aircraft in-flight, often without changing the physical properties of the aircraft. It allows adjustments of response 
characteristics and handling qualities. An aircraft equipped with such a system can mimic the response of other aircraft, lowering the cost of pilot training. Such a system can also be used for control law development and handling quality research [1].

In the early years, variable stability simulators were used to design control logic for different flight situations, like landing on an aircraft carrier [2]. In the 1970s and 1980s, focus moved from specific flight situations or aircraft configurations to testing conceptual control logic of novel aircraft. More recent developments show the possibility for the in-flight simulator to follow the dynamics of different aircraft [2]. Moreover, a trend within industry is the replacement of conventional hydraulic actuation with electrical actuation. Electrical actuators generally have a lower performance compared to hydraulic actuators [3]. This requires new control methods to achieve the desired handling qualities.

Different methods have been investigated to modify the handling qualities using variable stability. The response feedback method, which feeds back the aircraft response to modify the control inputs, was researched by Mirza et al. [4]. They were successful in modifying the handling qualities, but were not able to select them. A method that enables selection of handling qualities is model-following [5]. It forces an aircraft to perform like a predetermined model [6] [7]. A drawback of both methods is that multiple linear controllers are required, since an aircraft is a non-linear system. The controllers have to be gain-scheduled in the operating regime of the aircraft, which is not beneficial.

The non-linearities can be accounted for by using dynamic inversion, which uses the differentiation of the aircraft output until the control input appears in the derivative [8]. Ko et al. [9] developed a variable stability system based on this principle, where the pitch, roll, yaw and normal load could be matched [10]. However, their research was limited to offline simulations and linearised aircraft models. Miller [11] introduced nonlinear aircraft models and flew the variable stability system in a modified F-18. His implementation required full non-linear aircraft models and is thus constrained by the availability and accuracy of these models.

Incremental non-linear dynamic inversion (INDI) is a novel development in the field of dynamic inversion. It uses the aircraft accelerations and converts them to equivalent pitch, roll or yaw inputs using a moment effectiveness parameter [12]. A full non-linear aircraft model is not required anymore. Germann [13] designed a variable stability system using INDI for the T-6A Texan II aircraft. He linearised the moment effectiveness parameter which limited the performance of his system and he only tested the system in offline simulation. Grondman et al. [14] developed an INDI controller for the Cessna Citation II business jet. Their controller was flown successfully but was not designed for variable stability.

These developments indicate the potential of INDI to achieve a variable stability system. It is beneficial over other techniques since it requires less gain scheduling, it does not require a full non-linear model of the aircraft dynamics and it enables the selection of handling qualities. The design and implementation of a variable stability system using INDI has not been attempted in previous research. The purpose of this paper is twofold. First it is investigated if INDI can be used to create a variable stability in-flight simulator. This will be tested in offline simulation, a simulator experiment and in the Cessna Citation II business jet. Secondly, the limits of such a system will be investigated. The proposed system is expected to be limited by accuracy of the moment effectiveness and performance of the actuation system. The fly-by-wire actuation system in the Cessna Citation II is based on its existing autopilot, inheriting the limited performance and safety considerations [15]. The control authority of the autopilot is restricted due to limitations in torque which are imposed on the actuator servo motors. Determining the limitations of the system is one of the challenges of this research.

The paper is structured as follows. First some additional background information is presented in Section II. Second, the controller design is explained in Section III. The controller is tested in offline simulations in Section IV, in a flight simulator experiment in Section V and finally validated in real flight tests in Section VI.

\section{Background}

\section{A. Variable Stability}

\section{Response feedback}

Response feedback is the most basic method to achieve a variable stability system. It uses the response of an aircraft and feeds it back into the aircraft to modify the control inputs. A mathematical description of this system is given by Nelson [16] and the solution technique for achieving a target response is presented by Bryson and Ho [17] using the Ricatti equations. Mirza et al. [4] found that response feedback is effective in changing the stability and handling qualities. Handling qualities that are changed by response feedback could be identified by the pilots in their experiment, even in a fixed-base simulation.

\section{Model-following}

A second method to achieve a variable stability system is called the model-following technique. The response of an aircraft is changed so it performs like a predetermined model. This is beneficial when designing aircraft to meet the specifications determined by regulations. Two different strategies, implicit or explicit model-following, are used to design model-following control systems, as originally described by Armstrong [5], O'Brien and Broussard [6] and Kreindler and Rothschild [7]. The implicit model-following technique is a basic method where gains are selected to reach the desired behaviour. In explicit model-following, the model to be followed is placed inside the control logic as a feedforward compensator. 


\section{Dynamic inversion}

The previously presented control methods share the same drawback. Since an aircraft is a non-linear system, the described design tools require multiple linear controllers, which have to be gain-scheduled in the operating regime of the aircraft. Alternatively, these non-linearities can be accounted for with a technique called dynamic inversion. Slotine and Li [8] developed the initial concept, which uses differentiation of the output until the control input appears in the derivative.

Such a linear dynamic inversion was implemented by Ko et al. [9] in a variable stability system. They used a model of the Korean next-generation fighter and used linear dynamic inversion to follow the dynamics of the F-16 fighter aircraft in offline simulations. Ko and Park [18] introduced an updated version of their system, which included a switching mechanism between the normal flight computer and the dynamic inversion model. Finally Ko and Park [10] introduced the normal load to their initial variable stability control system, that only could follow pitch, roll and yaw. However, they do not discuss the main limitation of linear dynamic inversion. The performance of the linear dynamic inversion is constrained by the performance of the linear model of the aircraft. This linear model is, in turn, dependent on both the accuracy of the model and the operating point it is linearised at.

Non-linear dynamic inversion solves some of these issues. It uses a model of the aircraft non-linear dynamics, in contrast to the previously used linear approximation. In practice, this means that full look-up tables have to be implemented in the controller. Miller [11] shows such an implementation for the FAST system from NASA, with hardware in the loop simulation experiments. However, recent developments within dynamic inversion show that a full model of an aircraft is no longer required in the loop.

\section{Incremental non-linear dynamic inversion}

Incremental non-linear dynamic inversion (INDI) uses the advantages from the dynamic inversion, but does not require the full non-linear aircraft model. The development of INDI can be traced back to Smith [12]. He acknowledges the issues with the non-linear dynamic inversion and proposes a new method using acceleration sensors. The accelerations are converted to an equivalent pitch, roll or yaw input using a moment effectiveness parameter (based on the moment due to control surface deflection and the aircraft inertia). He demonstrated that the INDI method provides considerable robustness regarding authority limits, sensor noise and significant variations within the control law (mainly inertia and control powers).

The combination of the actuator position sensor and the acceleration sensors ensure that angular accelerations are matched robustly. Germann [13] developed a variable stability system for the T-6A Texan II for the American Airforce based on INDI. He showed in offline simulations that the Texan II matches the dynamics of the F-16 aircraft. Bacon and Ostroff [19] used the INDI method to design a re-configurable flight control system in case of system failure. They acquired the angular accelerations by combining linear accelerations and differentiating the angular rates. This created noise and was considered to be sufficient but not optimal since the differentiation also introduced some time delays. Cox and Cotting [20] continued the development of the ideas from Smith, Bacon and Ostroff. They implemented the INDI logic in the ground-based simulators at NASA Dryden. The main takeaway from their work is that INDI is viable for real-time aircraft control and simulation, not just in an offline simulation.

Smeur et al. [21] [22] showed that the INDI controller performs better regarding gust resistance, compared to a normal Proportional Integral Derivative (PID) controller. Grondman et al. [14] developed an INDI controller for the Cessna Citation II, which was based on angular rate control. Their controller was flown successfully in the Cessna Citation II. However, their controller was not designed for variable stability.

\section{B. Handling Qualities}

Handling qualities are defined as "those qualities or characteristics of an aircraft that govern the ease and precision with which a pilot is able to perform the tasks required in support of an aircraft role" [23]. This definition shows a clear combination of performance of the pilot and the aircraft acting together as a system for specific manoeuvres of the aircraft.

\section{Control Anticipation Parameter}

The Control Anticipation Parameter (CAP) is a longitudinal handling quality criterion which focuses on the short period pitch aircraft response. It was originally introduced by Bihrle [24], after which it became one of the criteria to measure longitudinal handling qualities, defined in the MILSTD-1797A, Flying Qualities of Piloted Aircraft [25]. It is expressed as the ratio of initial pitch acceleration (second derivative of $\theta$ ) to the normal acceleration in steady state $n_{z_{s s}}$ :

$$
C A P=\frac{\ddot{\theta}(0)}{n_{z_{s s}}}
$$

Bischoff [26] argued that the CAP can be calculated from the short period characteristics of the aircraft. This method only holds for aircraft with a general second order longitudinal response as given in Equation 2. Based on this assumption, Bischoff rewrites Equation 1 to Equation 3:

$$
\begin{gathered}
\frac{q}{\delta_{e}}=\frac{K_{\theta}\left(s+\frac{1}{T_{\theta_{2}}}\right)}{s^{2}+2 \zeta_{s p} \omega_{s p} s+\omega_{s p}^{2}} \\
C A P \approx \frac{\omega_{n_{s p}}^{2}}{n / \alpha} \approx \frac{\omega_{n_{s p}}^{2}}{\frac{V}{g} \frac{1}{T_{\theta_{2}}}}
\end{gathered}
$$

When the CAP is used as a handling quality criterion, it is always indicated in combination with the short period damping ratio. This criterion is categorised into different flight phases (A, B, C) and aircraft classes (I, II, III, IV). Within these categories, another segmentation exists into three different levels of acceptability (Level 1, 2 and 3).

- Level 1: Flying qualities are adequate for mission flight,

- Level 2: Flying qualities are adequate to accomplish the mission with increased pilot workload, and 
- Level 3: Flying qualities cause inadequate mission effectiveness due to excessive pilot workload.

\section{Low Order Equivalent System}

The combined actuator and aircraft dynamics are of higher order compared to the second order longitudinal response as assumed by Bischoff. Di Franco [27] was one of the first to research the effects of Higher Order System (HOS) dynamics on the longitudinal handling qualities. He determined that the aircraft short period response could be represented by a second-order equivalent term and a time delay. This concept was further developed into the Low Order Equivalent System (LOES) by Hodgkinson et al. [28]. They created an equivalent low order system by matching the complex frequency response of the higher order systems. In this way, a HOS can be represented as a low order system with a time delay term.

There are several techniques available to determine the LOES and their advantages and disadvantages are discussed by Mirza et al. [4]. The most robust method is the Output Error and Equation Error approach developed by Morelli [29]. The LOES method uses the equation error and output error parameter estimation in the frequency domain. They are two separate parameter estimation methods. The equation error method can estimate the parameters by fixing the time delay to a specific initial condition, whereas the output error method requires initial estimates for all parameters. However, the equation error method does not guarantee a global minimum. That is why Morelli suggested using the parameters from an initial equation error estimation as a guess for the output error estimation. This results in the estimation with the lowest error.

\section{Cooper-Harper Rating}

The Cooper-Harper rating scale is one of the accepted standards for subjective handling quality measurement. Cooper and Harper [23] developed a decision tree rating scale as a tool to assess aircraft handling quality. The scale takes qualitative pilot comments and translates them into a quantitative scale from 1 to 10 . It can be used in experiments to find the handling qualities as experienced by the pilots.

\section{Controller Design}

\section{A. Requirements}

Before the initial design of the controller can be created, some requirements for this controller are determined. First, the implementation of the controller should be flexible, since the variable stability platform should be used for multiple in-flight simulation purposes. This implies that it can be used for multiple goals without having to change the basic logic. To enable this, an inner loop and outer loop have been defined, where the functionality of the inner loop is independent of the outer loop. The outer loop can be used to define the different control goals.

Secondly, the controller should take into account the limitations of the system dynamics, being the actuators and aircraft dynamics, respectively. This is most important for the experiment in the simulator since this experiment should give an indication of the theoretical performance of the in-flight simulator. The fly-by-wire system will impose limitations on the in-flight simulation capabilities, combined with the physical limitations of the aircraft dynamics.

Both requirements lead to the schematic block diagram of the controller structure, which is shown in Figure 1.

\section{B. Inner Loop}

\section{INDI}

The main benefit of the INDI method is that the INDI controller uses information from the system dynamics to adapt the moment effectiveness dependent on the flight condition. This way, parts of the aerodynamic moment $M_{A}$ can be neglected in the model. The aerodynamic moment can be split into three main components, being the moment induced by thrust $M_{T}$, the moment induced by the control effectors $M_{c}$ and the airframe dependent moment $M_{a}$.

It is assumed that the moment induced by the thrust can be neglected. The airframe dependent moment and the moment induced by the control effectors are described by Equation 4 and Equation 5, respectively. In these equations $V_{\text {tas }}$ is the true airspeed, $\rho$ is the air density, $b$ is the wing span, $\bar{c}$ is the mean aerodynamic chord, $S$ is the wing surface area and $C_{*}$ are the aircraft's dynamic coefficients. These dynamic coefficients are defined by the system dynamics and retrieved from the aircraft model.

$$
\begin{gathered}
\boldsymbol{M}_{\boldsymbol{a}}=\frac{1}{2} \rho V_{\text {tas }}^{2} S\left[\begin{array}{c}
b C_{l_{a}} \\
\bar{c} C_{m_{a}} \\
b C_{n_{a}}
\end{array}\right] \\
\boldsymbol{M}_{\boldsymbol{c}}=\frac{1}{2} \rho V_{\text {tas }}^{2} S\left[\begin{array}{ccc}
b C_{l_{\delta_{a}}} & 0 & b C_{l_{\delta_{r}}} \\
0 & \bar{c} C_{m_{\delta_{e}}} & 0 \\
b C_{n_{\delta_{a}}} & 0 & b C_{n_{\delta r}}
\end{array}\right]
\end{gathered}
$$

Equations 4 and 5 can be incorporated in the rotational dynamics of rigid-body aircraft over a flat non-rotating earth as given in the Newton-Euler equation (Equation 6), where $J$ is the inertia tensor as given in Equation 7. This leads to Equation 8.

$$
\begin{gathered}
\dot{\boldsymbol{\omega}}=\boldsymbol{J}\left(\boldsymbol{M}_{\boldsymbol{A}}+\boldsymbol{M}_{\boldsymbol{T}}-\boldsymbol{\omega} \times \boldsymbol{J} \boldsymbol{\omega}\right) \\
\boldsymbol{J}=\left[\begin{array}{ccc}
I_{x x} & 0 & -I_{x z} \\
0 & I_{y y} & 0 \\
-I_{x z} & 0 & I_{z z}
\end{array}\right] \\
\dot{\boldsymbol{\omega}}=\boldsymbol{J}\left(\boldsymbol{M}_{\boldsymbol{a}}+\boldsymbol{M}_{\boldsymbol{c}} \boldsymbol{u}-\boldsymbol{\omega} \times \boldsymbol{J} \boldsymbol{\omega}\right)
\end{gathered}
$$

The control law for INDI is obtained by taking a first-order Taylor expansion around the current point in time, which is denoted by the subscript 0 . This Taylor expansion can be found in Equation 9. 


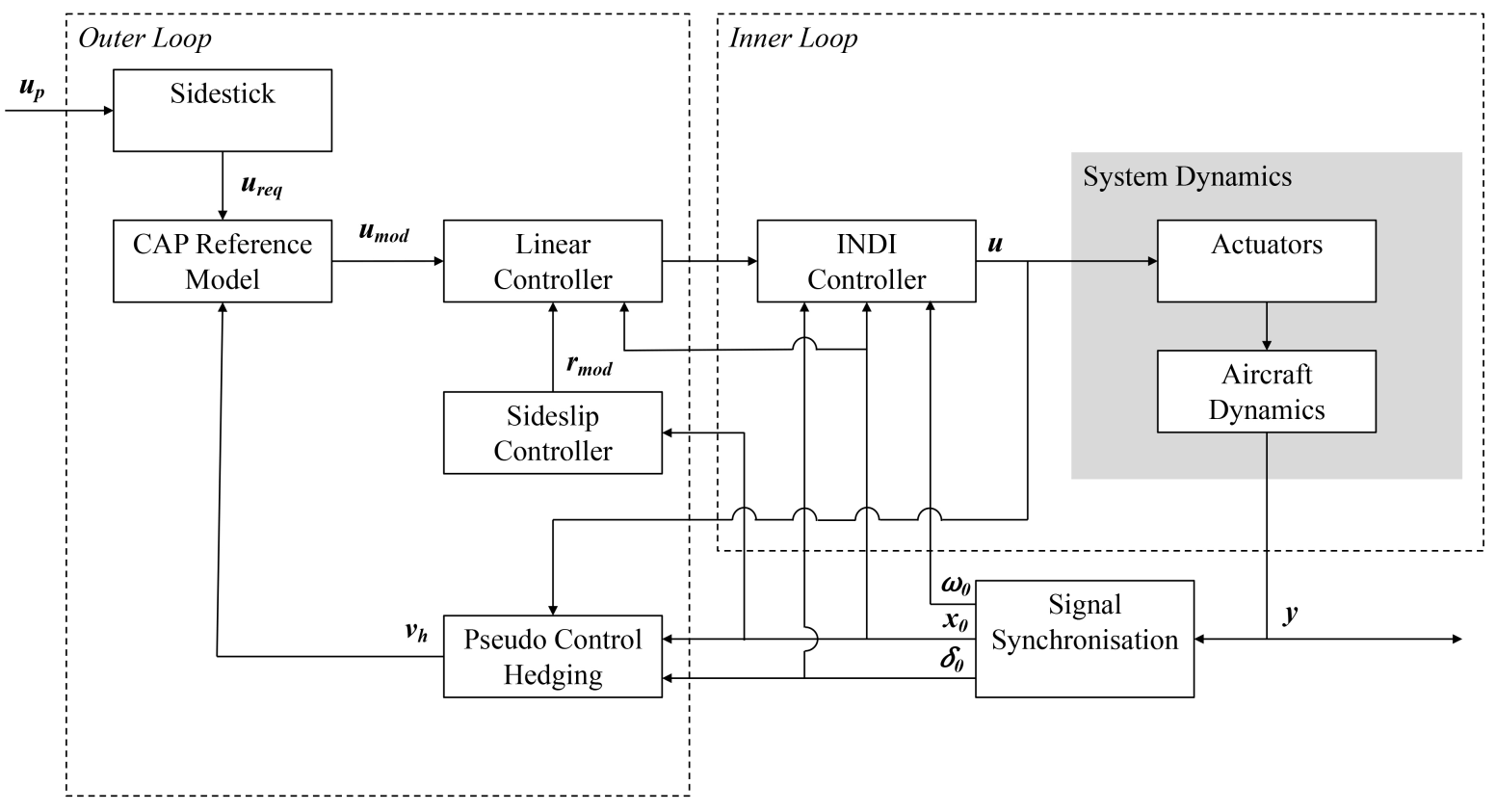

Fig. 1 Schematic block diagram of the controller structure

$$
\begin{aligned}
\dot{\omega} \approx \dot{\omega}_{0} & +\frac{\partial}{\partial \omega}\left(J^{-1}\left(M_{a}+M_{c} u-\omega \times J \omega\right)\right)\left(\omega-\omega_{0}\right) \\
+ & \frac{\partial}{\partial \boldsymbol{u}}\left(J^{-1}\left(M_{a}+M_{c} u-\omega \times J \omega\right)\right)\left(\boldsymbol{u}-\boldsymbol{u}_{\mathbf{0}}\right)
\end{aligned}
$$

The controller runs at $1000 \mathrm{~Hz}$, thus the time increments are relatively small. This fact, combined with assuming instantaneous control effectors $\left(\boldsymbol{\omega}-\boldsymbol{\omega}_{\mathbf{0}} \ll \boldsymbol{u}-\boldsymbol{u}_{\mathbf{0}}\right)$ and assuming that $\omega-\omega_{0}=0$, leads to a simplification of Equation 9 to Equation 10, where $\Delta \boldsymbol{u}=\boldsymbol{u}-\boldsymbol{u}_{\mathbf{0}}$ :

$$
\dot{\omega} \simeq \dot{\omega}_{0}+J^{-1} M_{c} \Delta u
$$

Inversion of Equation 10 leads to the INDI control law as implemented in the controller. This results in Equation 11, where the input of the linear controller $v_{\boldsymbol{e}}$ replaces $\dot{\omega}$ and the current control surface deflections $\boldsymbol{\delta}_{\mathbf{0}}$ are added. A schematic overview is given in Figure 2.

$$
u=M_{c}^{-1} J\left(v_{e}-\dot{\omega_{0}}\right)+\delta_{0}
$$

\section{System Dynamics}

The system dynamics are based on the DASMAT model of the Cessna Citation 500 described by Borst [30]. The DASMAT model is preferred over the newer Cessna Citation II model developed by Van den Hoek et al. [31], since it also includes the moments of the trim tabs. These moments are relevant when the model has to be trimmed towards steady, straight flight with the lowest possible hinge moment in the elevator. A low hinge moment in the elevator is required to maximise the performance of the limited fly-by-wire system.

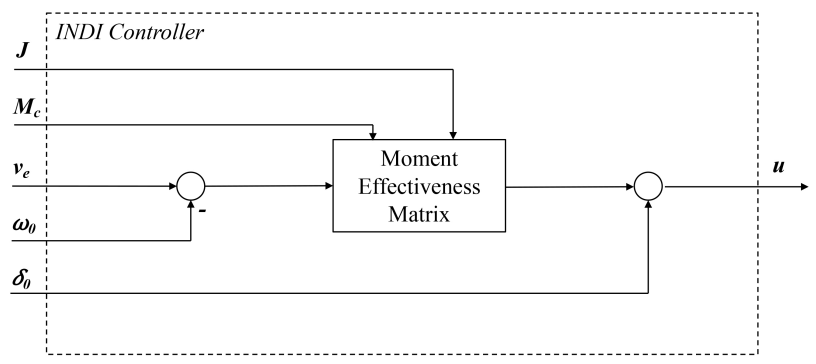

Fig. 2 Schematic block diagram of the INDI controller

The non-linear actuator model from Lubbers [15] is implemented in this DASMAT model. An alternative to this non-linear actuator model is based on a first-order approximation performed by Grondman et al. [14]. To be able to use both the non-linear actuator model and a more basic approximation of the actuators, a switch is built inside the model. It has been updated to make use of the saturation flags from the full actuator model by Lubbers [15], to ensure the appropriate saturation deflections.

During the flight test experiment, the DASMAT model is still used to determine the moment induced by the control effectors. However, the mass model has been updated to include the appropriate inertias and masses from the Citation II. The outputs from the aircraft sensors are synchronised and filtered using basic first-order low-pass filters, except for the angular acceleration which is filtered using a second-order low-pass filter $(\omega=20[\mathrm{rad} / \mathrm{s}]$ and $\zeta=1)$. These outputs are used in the remainder of the controller. 


\section{Outer Loop}

\section{Sideslip Controller}

The Cessna Citation II fly-by-wire hardware does not include a yaw input device, which means that coordinated flight can only be achieved when the sideslip is controlled. This has been recognised by Grondman et al. [14], who developed a PI controller to control the sideslip to zero. This is described in Equation 12, where $K_{\beta}=1.93, K_{\beta_{I}}=0.977$ and $w=V_{\text {tas }} \sin \alpha \approx V_{\text {tas }} \alpha$.

$$
r_{\text {mod }}=\frac{1}{V_{\text {tas }}}\left(w p-f_{y}\right)\left(\frac{1}{s} K_{\beta_{I}}\left(\beta_{c m d}-\beta\right)-K_{\beta} \beta\right)
$$

\section{Pseudo-Control Hedging}

Johnson and Calise [32] developed a technique to compensate for actuator limitations by modifying the reference model dynamics. This technique is called Pseudo-Control Hedging (PCH). $\mathrm{PCH}$ is implemented in the controller to account for the actuator saturation, which is expected due to the limited fly-by-wire system performance. PCH uses the inverse of the moment effectiveness matrix to correct for the differences between the commanded deflection by the INDI controller and the actual deflection of the actuators. This provides the hedging signal as indicated in Equation 13:

$$
v_{h}=M_{c} J^{-1}\left(u-\delta_{0}\right)
$$

Looye et al. [33] activated the $\mathrm{PCH}$ only when saturation occurred, whilst Grondman et al. [14] always kept the PCH active. In the latter case, the reference logic is also corrected for limited actuator speed instead of only the actuator position and this option is therefore preferred.

\section{CAP Reference Logic}

The CAP reference logic in pitching motion is designed to ensure a requested CAP and damping using the second order approximation as determined by Bischoff [26]. The linear controller uses $\boldsymbol{u}_{\boldsymbol{m o d}}$ as an input, which is essentially a reference pitch and roll rate. Yaw is not controlled to zero by the controller, thus it is not present in the CAP reference logic. To convert the requested input $\boldsymbol{u}_{\boldsymbol{r} \boldsymbol{e} \boldsymbol{q}}$ to these reference rates, an estimation of both the pitch gain $K_{\theta}$ and the longitudinal coefficient $T_{\theta_{2}}$ are required as given in Equation 2. To achieve this estimation, the method from Morelli [29] is used. Since the CAP and damping are design parameters, Equation 2 can be rewritten to provide the final CAP reference model Equation 14. The corresponding coefficients are given in Equation 15.

$$
\begin{gathered}
\frac{u_{\text {mod }_{q}}}{u_{r e q_{q}}}=\frac{a_{1} s+a_{2}}{s^{2}+b_{1} s+b_{2}} \\
a_{1}=K_{\theta} \quad a_{2}=\frac{K_{\theta}}{T_{\theta_{2}}} \\
b_{1}=2 \zeta_{q} \sqrt{\mathrm{CAP} \cdot \frac{V}{g} \frac{1}{T_{\theta_{2}}}} \quad b_{2}=\frac{\mathrm{CAP}}{T_{\theta_{2}}} \frac{V}{g}
\end{gathered}
$$

In rolling motion different reference dynamics are used as given in Equation 16, with the coefficients defined as in Equation 17. Grondman et al. [14] performed a design optimisation for these coefficients, resulting in $\zeta_{p}=1$ and $\omega_{p}=1.35[\mathrm{rad} / \mathrm{s}]$.

$$
\begin{gathered}
\frac{u_{\text {mod }_{p}}}{u_{r e q_{p}}}=\frac{c_{1}}{s^{2}+d_{1} s+d_{2}} \\
c_{1}=\omega_{p}^{2} \quad d_{1}=2 \zeta_{p} \omega_{p} \quad d_{2}=\omega_{p}^{2}
\end{gathered}
$$

\section{Sidestick}

The inputs from the pilot have to be scaled before they can enter the controller. Since both roll and pitch have different reference logic, both axes need different gains. The initial values were determined using a pilot model, where the gain was changed until the open loop crossover frequency $\omega_{c}$ was equal to $2[\mathrm{rad} / \mathrm{s}]$. This led to the following standard stick gains: Pitch $K_{q}=0.0125$ and roll $K_{p}=0.65$.

\section{Linear Controller}

The linear controller was designed to follow the reference model as closely as possible. For the pitch and roll channel, the error for the state and the first derivative are controlled. In addition, a feedforward gain was added to the second derivative. The result can be seen in Equations 18-19:

$v_{\dot{\phi}}=\left(K_{\phi}+\frac{K_{\phi_{I}}}{s}\right)\left(u_{\bmod _{p}}-\phi\right)+K_{\dot{\phi}}\left(\dot{u}_{\bmod _{p}}-\dot{\phi}\right)+K_{\ddot{\phi}} \ddot{u}_{\bmod _{p}}$

$v_{\dot{\theta}}=\left(K_{\theta}+\frac{K_{\theta_{I}}}{s}\right)\left(u_{\bmod _{q}}-\theta\right)+K_{\dot{\theta}}\left(\dot{u}_{\text {mod }_{q}}-\dot{\theta}\right)+K_{\ddot{\theta}} \ddot{u}_{\text {mod }_{q}}$

Sideslip is controlled in the yaw channel, without any pilot input:

$$
v_{r}=K_{r}\left(r_{\text {mod }}-r\right)
$$

Grondman et al. [14] performed a robust parameter synthesis to optimise these gains. However, their design requirements were different, since they did not require exact model-following. A linear parameter grid search was performed to find the combination of gains that enabled better model-following performance. The results of this analysis are summarised in Table 1.

\section{Offline Simulation}

Multiple offline simulations were performed to investigate the performance of the controller. In addition, the offline simulations were used to determine the optimal flight condition considering the moment effectiveness and actuator saturation. This led to an initial estimation of the system limitations. 
Table 1 Linear controller gains

\begin{tabular}{llll}
\hline \hline Roll & & Pitch \\
$K_{\phi}$ & 5.51 & $K_{\theta}$ & 7.76 \\
$K_{\phi_{I}}$ & 1.34 & $K_{\theta_{I}}$ & 0.50 \\
$K_{\dot{\phi}}$ & 4.80 & $K_{\dot{\theta}}$ & 4.80 \\
$K_{\ddot{\phi}}$ & 1.05 & $K_{\ddot{\theta}}$ & 0.70 \\
\hline Yaw & & & \\
$K_{r}$ & 1.62 & & \\
\hline \hline
\end{tabular}

\section{A. CAP and damping performance}

To investigate the performance in placing the CAP and damping parameters, step inputs were given on the pitch axis in Figure 3. The same step input is given in both situations, but the settings for the CAP reference model are different. Figure 3a shows the response of the aircraft for a CAP of 0.6 and a damping of 1.0. Figure $3 \mathrm{~b}$ depicts the response for a $\mathrm{CAP}$ of 1.1 and a damping of 0.3 . It can be seen that the controller modifies the response of the elevator to match the requested dynamics as closely as possible. In both cases the $\mathrm{PCH}$ is enabled, which results in the difference between the commanded signal and the reference signal.

The matching performance of the $\mathrm{PCH}$ depends on a proper synchronisation of the signals. A transport lag exists between the commanded deflection and the actual deflection of the actuators, which resulted in an over-correction by the hedging signal. During the offline simulations and the fixed-base experiment this transport lag is relatively constant, but in real flight this lag may vary due to non-linearities. In addition, the hedging signal directly adapts the CAP reference logic. This implies that the handling qualities change when $\mathrm{PCH}$ is enabled, which is undesired behaviour. This should be taken into account when using PCH in the flight tests.

\section{B. Flight condition}

The offline model was used to investigate the initial flight condition that would result in the highest pitch and roll rates. This condition was limited by two main factors, being the moment effectiveness and the deflection of the actuators. The moment effectiveness is higher for high speeds and low altitudes, whereas the maximum deflection of the actuators is higher at low speeds and high altitudes. A controller in the fly-by-wire system allows command over the deflection angle of the control surfaces, by controlling the servo motor position. The transmission ratio between deflection of the control surface and position of the servo motor is not a fixed ratio. It is a function of the force on the actuator cables due to cable stretch. The control effectiveness therefore changes dependent on the flight condition. Combining these factors led to an initial condition of $4000[\mathrm{~m}]$ altitude and a true airspeed of $110[\mathrm{~m} / \mathrm{s}]$. At altitudes higher than approximately $4250[\mathrm{~m}]$, torque limiters are enabled for the fly-by-wire system. This was one of the main limiting factors when determining the optimal initial condition with respect to system performance.

\section{System limit prediction}

To determine the CAP and damping limits of the system, it is required to make an assumption regarding the time it takes before pilots experience differences in handling qualities due to actuator saturation. In addition, it is dependent on the behaviour of the pilot during the experiment. Some pilots might fly the aircraft with a higher gain which results in different crossover frequencies. The effect of both parameters on the system limits is investigated. It was found that the crossover frequency has a higher impact on the system limits. This is due to the actuator saturation that occurs more frequently and for a prolonged time when the crossover frequency is higher. For the initial system limit prediction it is assumed that pilots feel a difference in handling qualities when actuator saturation of 0.2 seconds or longer occurs. This assumption will be updated after the simulator experiment, since this gives an opportunity to investigate a relation between experienced handling qualities and the actuator saturation.

Using a pilot model with different crossover frequencies it was investigated for which combinations of CAP and damping actuator saturation of 0.2 seconds would occur for the control task of the fixed-base simulator experiment. Figure 4 illustrates the effect of two different crossover frequencies as a function of CAP and damping ratio for the defined control task. The pink dots represent the initial conditions chosen for the simulator experiment, which will be explained in more detail in the next section.

\section{Experiment 1: Fixed-base Simulator}

A fixed-base simulator experiment is performed to investigate what the system limits are due to the actuator saturation for a specific control task. In addition, it is investigated whether pilots can feel the difference between simulating just the reference model, and simulating the complete non-linear dynamics of the reference model, controller and aircraft.

\section{A. Method}

\section{Apparatus}

The experiment was performed in a fixed-base, part-task flight simulator at Delft University of Technology's Human Machine Interaction Laboratory (HMI lab). Figure 5 depicts the layout of the aircraft configuration. It consists of an adjustable aircraft seat, two 18 inch LCD panels that show the instrumentation and three DLP projectors which are used to display the outside visuals. Participants used an electrohydraulic sidestick to control the motion in pitch and roll. Clouds were added to the simulation to enhance the experience of forward motion for the pilots.

\section{Software}

Since multiple experiments were performed on different platforms, DUECA by van Paassen et al. [34] is used as a basic software platform. DUECA has been developed to act as a middleware layer between hardware (simulators/research aircraft) and the actual control software itself. It is able 

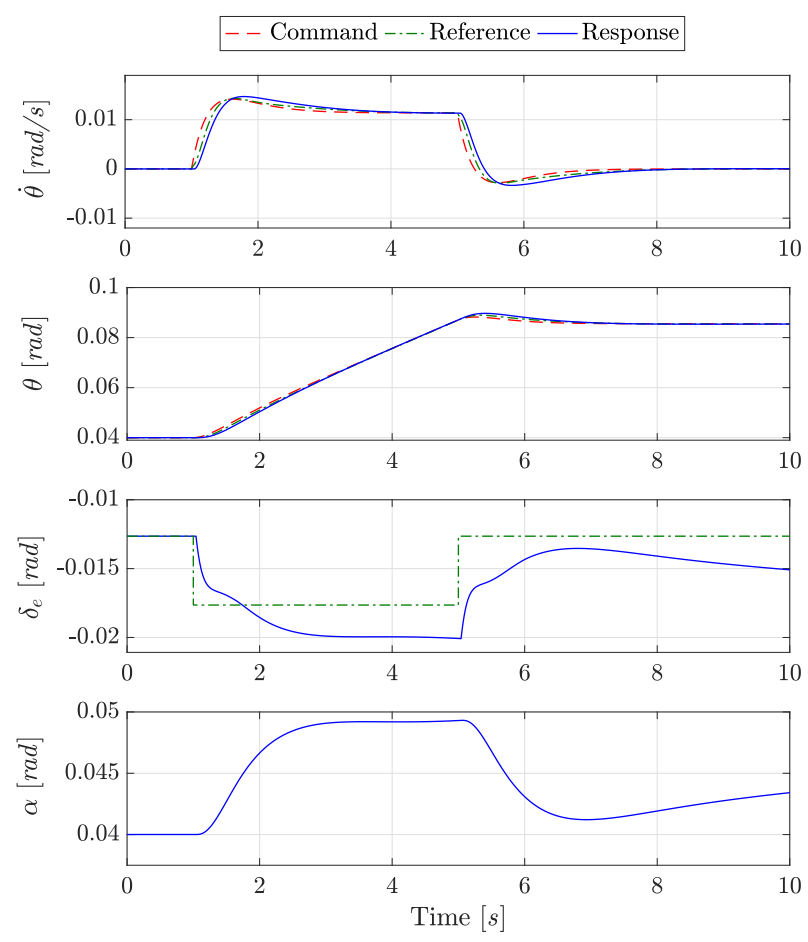

(a) $\mathrm{CAP}=0.6$; Damping $=1.0$
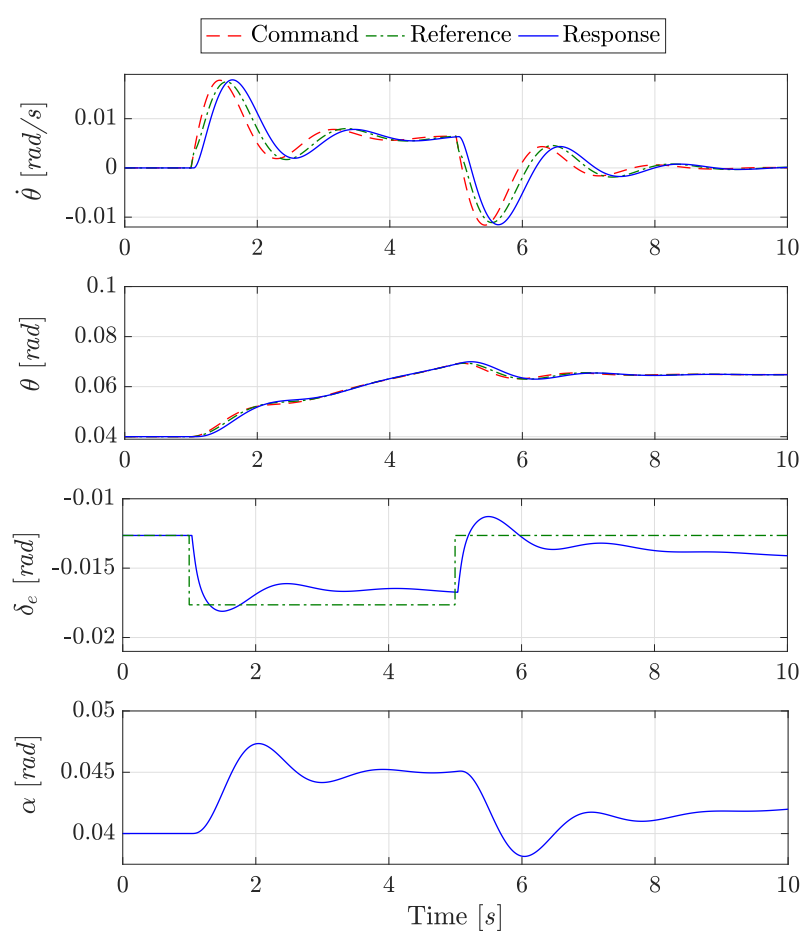

(b) CAP $=1.1$; Damping $=0.3$

Fig. 3 Aircraft pitch response for different CAP and damping values, with PCH enabled

to synchronise data from different sources and it combines processes that are running at different frequencies. Different modules can be selected within the software, which makes the implementation on different platforms easier. In addition, controllers developed in the MATLAB/Simulink environment are easily converted and implemented to control either the simulators or the Cessna Citation II laboratory aircraft.

\section{Subjects and Instruction to Subjects}

Eight pilots participated in the experiment (Table 2). They were instructed to control the aircraft's rate of climb to 1000 [feet/min] and $-1000[$ feet/min], with a performance band around the commanded rate of climb of $10 \%$. Every ten seconds they were commanded to switch between ascent and descent.

\section{Independent Variables}

The experiment consisted of one independent variable, being the model that the pilot is using to fly the simulation. This independent variable has two conditions, the reference model and the full model. The reference model (CAPR) uses the response from the CAP reference model and sends these dynamics directly to the simulator. The full model (FULL) also includes the controller and the aircraft dynamics. This is represented in the block diagram in Figure 6. The CAP reference model is not limited by actuator saturation, whereas the full model includes these limitations.
Table 2 Characteristics of the pilot subjects

\begin{tabular}{|c|c|c|c|c|}
\hline Pilot & Age & Sex & Hours & Types of aircraft \\
\hline A & 52 & $\mathrm{M}$ & 12,000 & $\begin{array}{l}\text { Single engine; B777; B787; } \\
\text { Cessna Citation II }(1,000 \mathrm{~h})\end{array}$ \\
\hline B & 63 & M & 20,000 & $\begin{array}{l}\text { Single engine; B777; B787; } \\
\text { Cessna Citation I (500h) }\end{array}$ \\
\hline $\mathrm{C}$ & 59 & M & 3,700 & Single engine; F5; F16 \\
\hline $\mathrm{D}$ & 65 & M & 22,000 & $\begin{array}{l}\text { Single engine; B737; B747; } \\
\text { Cessna Citation I (50h) }\end{array}$ \\
\hline $\mathrm{E}$ & 42 & M & 3,700 & $\begin{array}{l}\text { Single engine; Cessna Cita- } \\
\text { tion II }(1,650 \mathrm{~h})\end{array}$ \\
\hline $\mathrm{F}$ & 46 & M & 2,200 & $\begin{array}{l}\text { Single engine; SA266/277; } \\
\text { Cessna Citation II }(1,300 \mathrm{~h})\end{array}$ \\
\hline G & 58 & M & 14,300 & $\begin{array}{l}\text { Single engine; Jet engine; } \\
\text { Cessna Citation II }(3,000 \mathrm{~h})\end{array}$ \\
\hline $\mathrm{H}$ & 52 & M & 1,300 & $\begin{array}{l}\text { Single engine; Jet engine; } \\
\text { Cessna Citation II }(6 \mathrm{~h})\end{array}$ \\
\hline
\end{tabular}

\section{Experimental Design and Procedure}

The experiment was designed to be full-factorial withinsubjects. The CAP conditions were selected to ensure that different system limitations, due to actuator saturation, could be experienced. This resulted in a CAP of 0.90, 0.45 and 0.25 .

The different damping conditions were selected to have all three levels of acceptability in the experiment. The original 


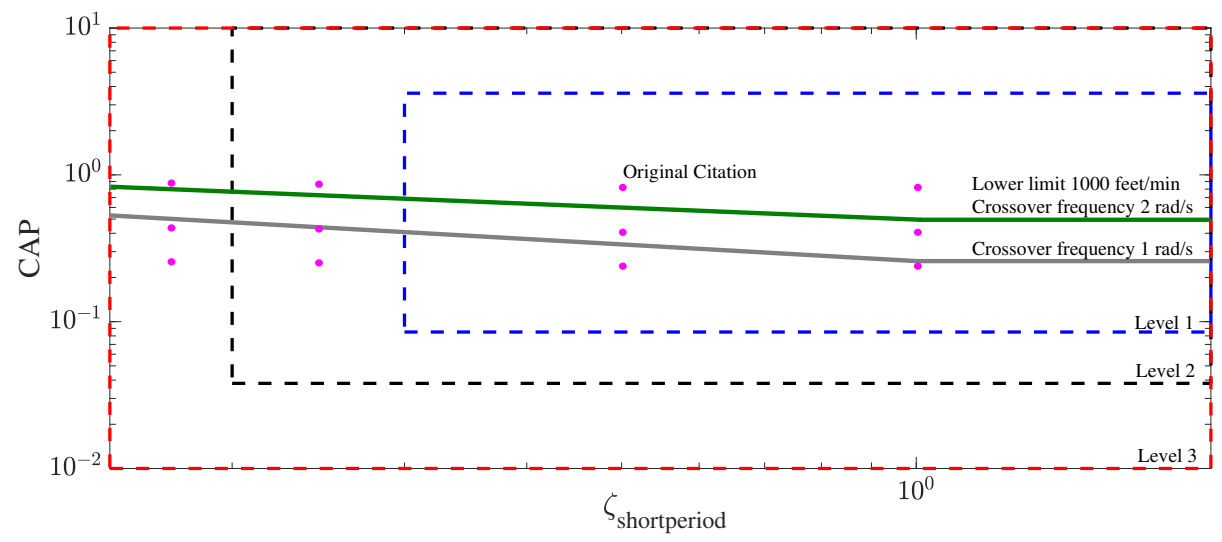

Fig. 4 Initial conditions displayed with predicted limitations from offline simulation

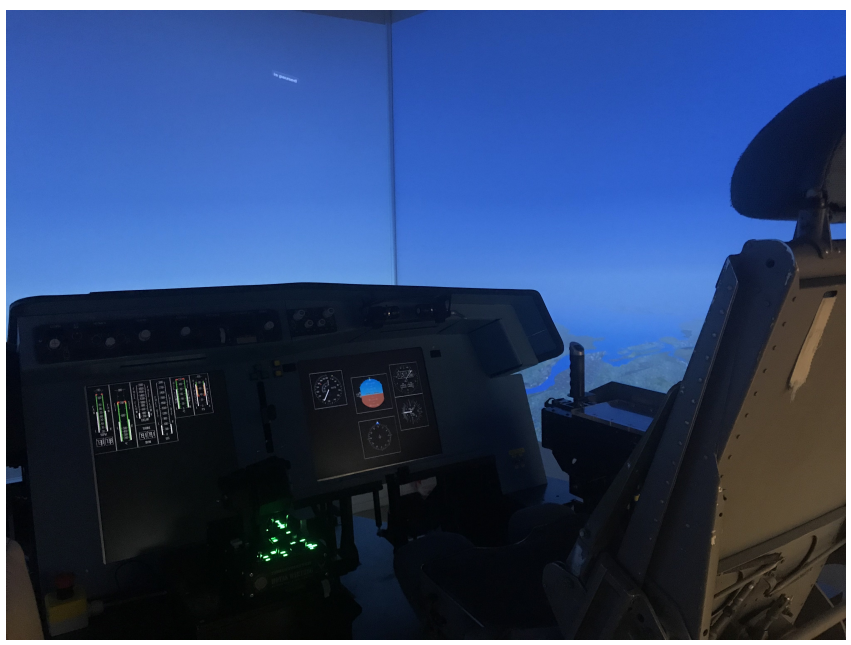

Fig. 5 Fixed-base simulator in aircraft configuration

damping of the Cessna Citation II was also included and this resulted in a damping of $0.15,0.25,0.5$ and 1.0. Combined with the initial CAP conditions, this yielded 12 conditions which are represented by the pink dots in Figure 4.

The initial conditions were flown for both the CAP reference model and the full model, resulting in a total of 24 runs. The 24 runs were divided into 4 sets of 6 runs, where for every set the damping coefficient remained constant, whilst the CAP and simulation model varied. Before every set, training runs were performed to remove the factor of learning from the data. These training runs were performed on the settings of the first run of the upcoming set. The experiment schedule for one of the pilots is shown in Table 3. Sets were varied over all pilots, including the start with the full or CAP reference model per set. The resulting experiment matrix required eight pilots. The initial flight conditions of all runs were defined according to the offline simulation $(4000[\mathrm{~m}]$ altitude and $110[\mathrm{~m} / \mathrm{s}]$ true airspeed).

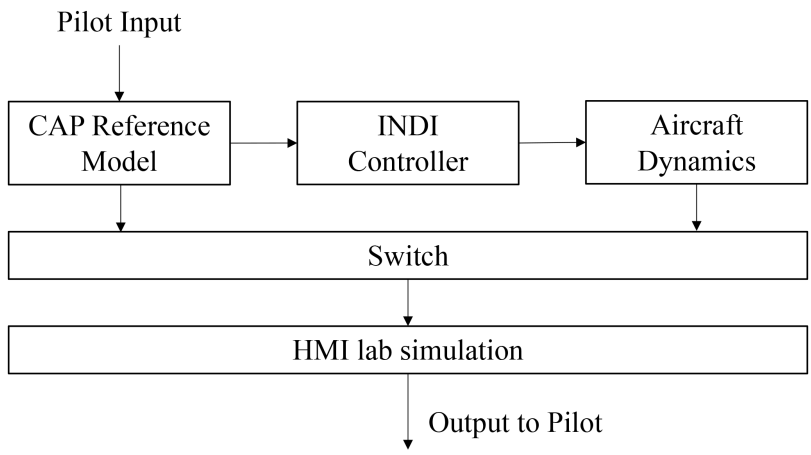

Fig. 6 Flow diagram concerning the independent variable with two conditions

\section{Dependent Measures}

The dependent measures are used to determine the differences between the conditions of the independent variable. In addition, they are also used to find the limits of the full model based on actuator saturation. The dependent measures are defined as follows:

- Cooper-Harper Rating Scale: The subjects had to give a Cooper-Harper rating after every run. The difference between the CAP reference model and the full model for the same initial condition is used to compare the results.

- Performance: The total time spent within the specified $10 \%$ bandwidth around the required rate of climb is used as performance indicator. The percentage of the total run time is referred to as the performance ratio.

- Control activity: An increased workload indicates that the pilot has a harder time flying the aircraft. The control activity during the simulation can therefore be used as workload measure. The control activity is measured by the variance of the input of the pilot. Since the main goal is to determine whether the pilots notice the change in longitudinal handling qualities, only the control activity in pitch is of interest.

- Actuator saturation: Actuator saturation is measured 
Table 3 Example of an experiment schedule for one pilot

\begin{tabular}{|c|c|c|c|}
\hline SET 1 & SET 2 & SET 3 & SET 4 \\
\hline Damp 0.15 & Damp 0.25 & Damp 0.50 & Damp 1.0 \\
\hline CAPR & FULL & CAPR & FULL \\
\hline CAP 0.9 & CAP 0.9 & CAP 0.9 & CAP 0.9 \\
\hline FULL & CAPR & FULL & CAPR \\
\hline CAP 0.9 & CAP 0.9 & CAP 0.9 & CAP 0.9 \\
\hline FULL & CAPR & FULL & CAPR \\
\hline CAP 0.45 & CAP 0.45 & CAP 0.45 & CAP 0.45 \\
\hline CAPR & FULL & CAPR & FULL \\
\hline CAP 0.45 & CAP 0.45 & CAP 0.45 & CAP 0.45 \\
\hline CAPR & FULL & CAPR & FULL \\
\hline CAP 0.25 & CAP 0.45 & CAP 0.25 & CAP 0.25 \\
\hline FULL & CAPR & FULL & CAPR \\
\hline CAP 0.25 & CAP 0.45 & CAP 0.25 & CAP 0.25 \\
\hline
\end{tabular}

since a correlation is expected between the actuator saturation and the differences in Cooper-Harper rating between the different models. Two different flags are used. The first one is logged when actuator saturation occurs and will be referenced as saturation flag in the remainder of this paper. The second one is logged when actuator saturation occurs and the requested input of the pilot is in the opposite direction of the current aircraft movement. In other words, a pilot might not feel saturation when the aircraft is moving in the direction as requested by the pilot. This flag is referenced to as the combined flag.

\section{Experiment hypotheses}

It is hypothesised that:

- H.1: The performance of the pilots is similar for both models if no actuator saturation is occurring. Performance is expected to be lower if pilots reach the saturation limits of the actuators. Since actuator saturation is expected during the experiment, the full model is likely to have lower performance compared to the CAP reference model.

- H.2: The control activity is higher for the full model, since the full model includes actuator saturation. This means that pilots will sometimes not experience the expected response from the aircraft.

- H.3: Pilots will give more similar Cooper-Harper ratings for a higher CAP, since the offline simulation showed that actuator saturation time is lower for these conditions. However, this depends on the crossover frequency of the pilot and the assumption that pilots only experience differences when a saturation time of more than 0.2 seconds occurs.

\section{B. Results}

\section{Cooper-Harper difference}

The difference in Cooper-Harper rating between both models is displayed in Figure 7. It can be seen that most runs were assessed with two points difference, which means that pilots experience the full model to have a worse rating compared to the CAP reference model. For only one run the full model was considered to be the better model. There was a statistically significant difference in Cooper-Harper difference depending on the initial CAP level, $\chi^{2}(2)=44.816, p<0.00$. Post hoc analysis was performed using the Wilcoxon signedrank tests with a Bonferroni correction (significance level of $p<0.02)$. Median perceived Cooper-Harper differences for the CAP of 0.90, 0.45 and 0.25 were 1 ( 0 to 2 ), 2 ( 2 to 4 ) and 2 (2 to 3.75), respectively. There were significant differences between the CAP 0.90 and 0.45 condition $(Z=-4.247, p<$ $0.00)$ and between the CAP of 0.90 and $0.25(Z=-4.430, p<$ $0.00)$. However, there was no statistically significant reduction in the perceived Cooper-Harper difference between CAP 0.45 and $0.25(Z=-0.540, p<0.59)$.

The difference in rating can be visualised using the aircraft response of two experiment runs. Figure 8 shows a run of the full model, where the pilot did not notice any differences compared to the CAP reference model (0 point Cooper-Harper difference). The first subplot shows the rate of climb and the performance bands (marked as two grey boxes). Furthermore, the pitch angle and elevator deflection are given. Finally, the pilot input is displayed. It can be seen that the longest sustained saturation time occurs in the black dotted box between 80 and 90 seconds (the previously mentioned saturation flag). Even though the pilot is experiencing saturation, the pilot still graded the full model similar to the CAP reference model.

Figure 9 shows another run of the full model, but here the pilot is noticing a six-point Cooper-Harper rating difference with respect to the CAP reference model. Saturation occurs more often and is sustained for a longer period of time. Similarly as before, the black dotted box between 40 and 50 seconds shows the saturation flag. However, the magenta box shows the combined flag, during which the pilot tries to steer the aircraft in a different direction, but the aircraft does not respond as requested.

The maximum saturation time for both the saturation flag and the combined flag can be plotted against the Cooper-Harper differences. This can be used to investigate the correlation between the maximum flag time and the difference in experienced handling qualities. The saturation flag is plotted in Figure 10, whereas the combined flag is displayed in Figure 11. The number behind the summation symbol represents the amount of data points in the respective column. It can be seen that the flag time means and standard deviations increase when the perceived Cooper-Harper rating difference increases. The saturation flag appears to have higher means compared to the combined flag. However, due to the limited number of data-points no statistically significant linear correlation could be found between the Cooper-Harper differences and their corresponding maximum flag time. 


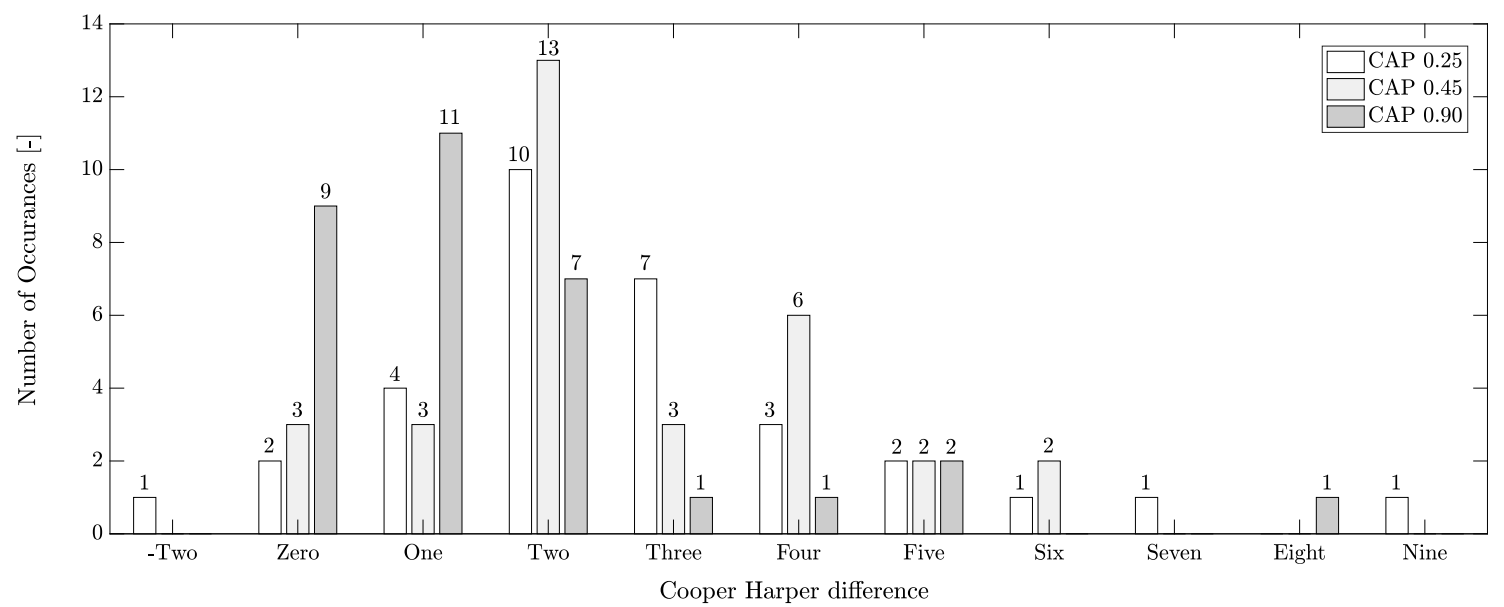

Fig. 7 Cooper-Harper difference between CAP reference model and the Full model as indicated by the pilots
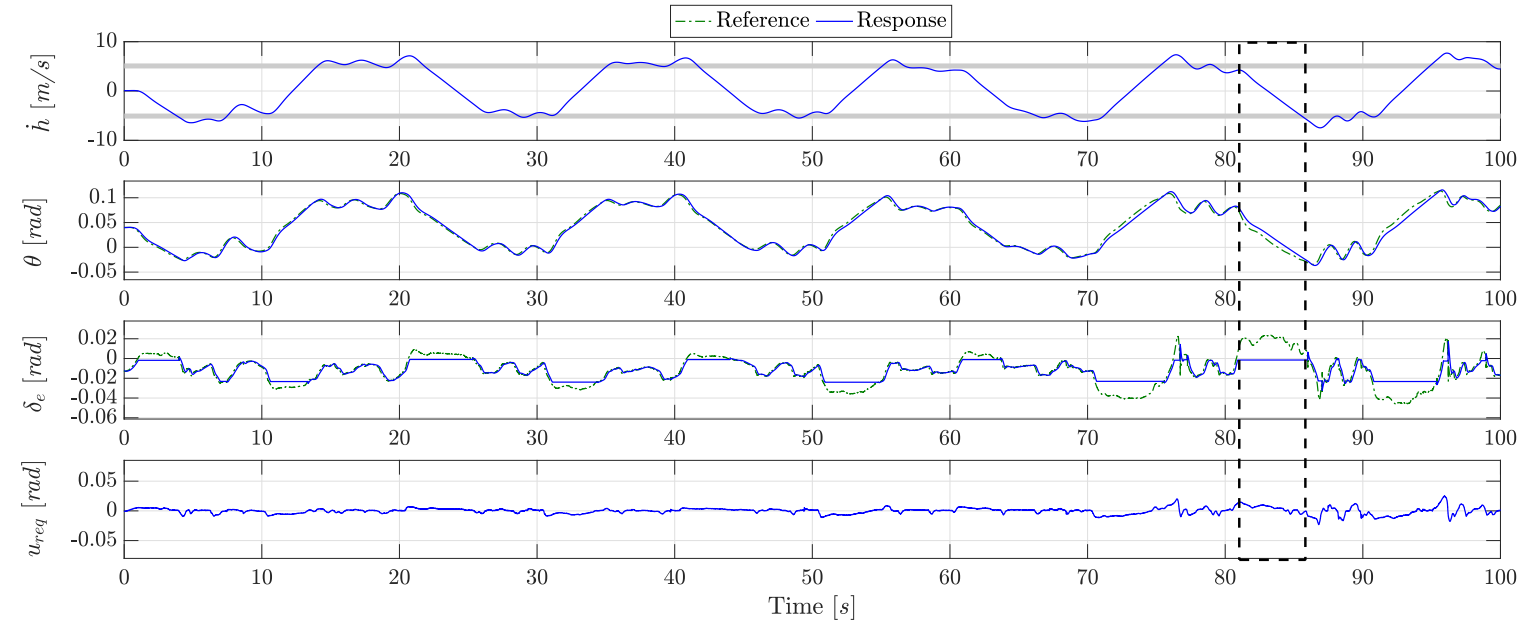

Fig. 8 Aircraft response with Cooper-Harper difference of 0pt (CAP 0.9; Damping ratio 0.25)

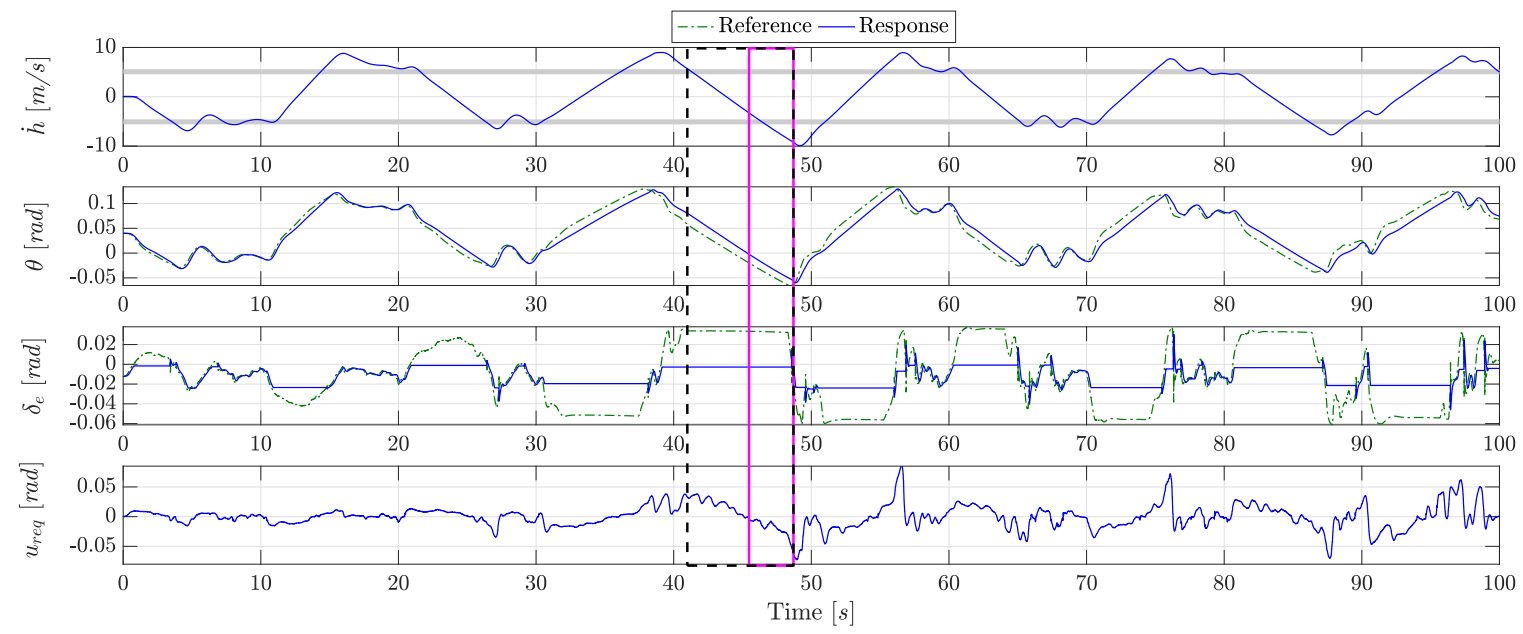

Fig. 9 Aircraft response with Cooper-Harper difference of 6pt (CAP 0.25; Damping ratio 1.0) 


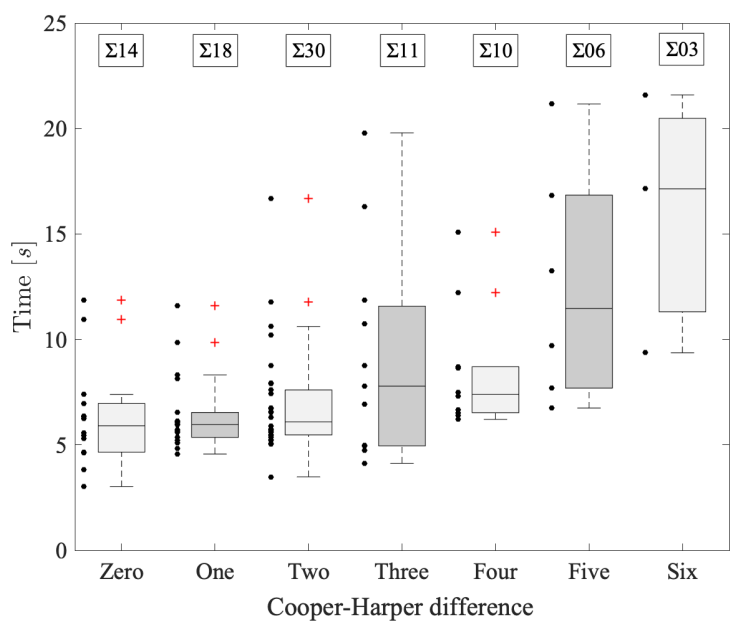

Fig. 10 Means and the $95 \%$ confidence limits of the saturation flag sorted by Cooper-Harper difference

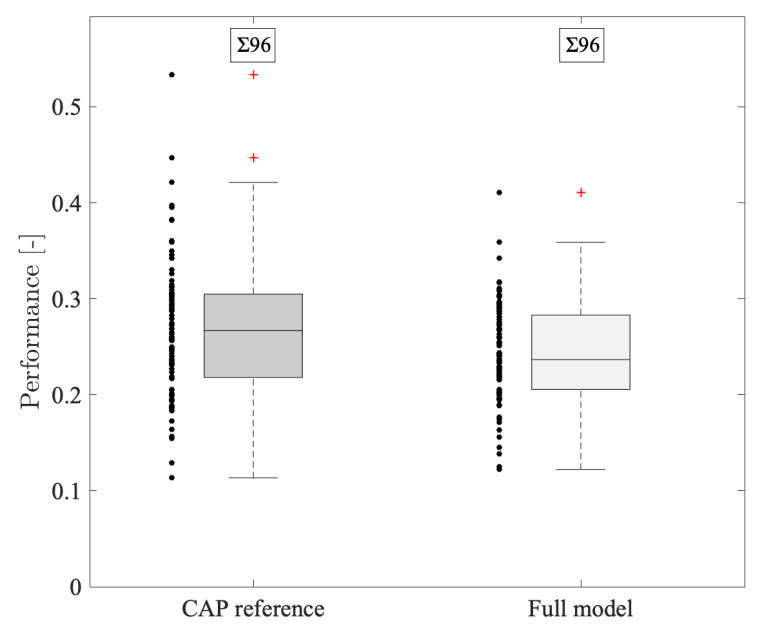

Fig. 12 Means and the $95 \%$ confidence limits of the performance

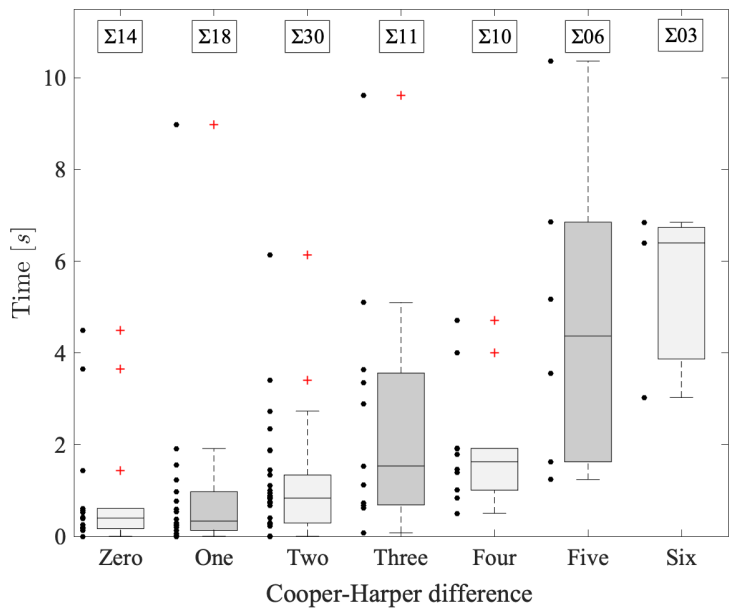

Fig. 11 Means and the $95 \%$ confidence limits of the combined flag sorted by Cooper-Harper difference

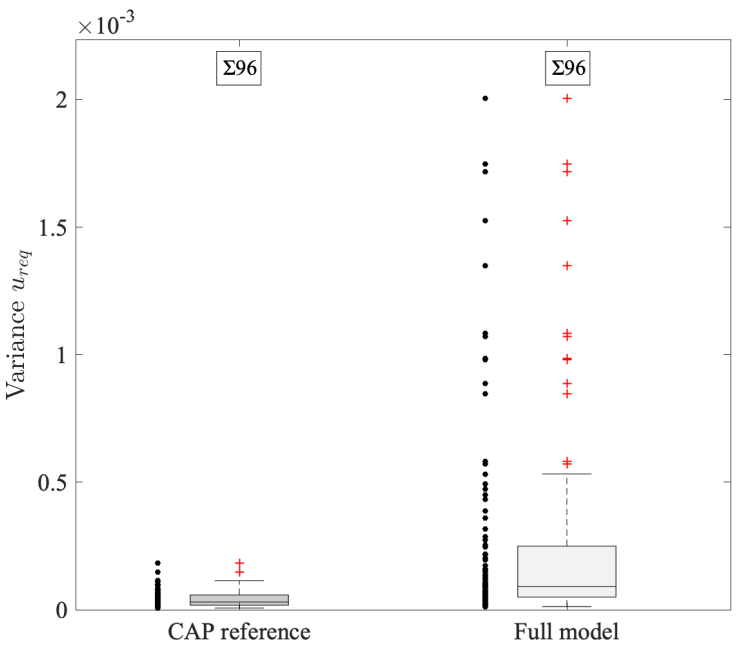

Fig. 13 Means and the $95 \%$ confidence limits of the control activity

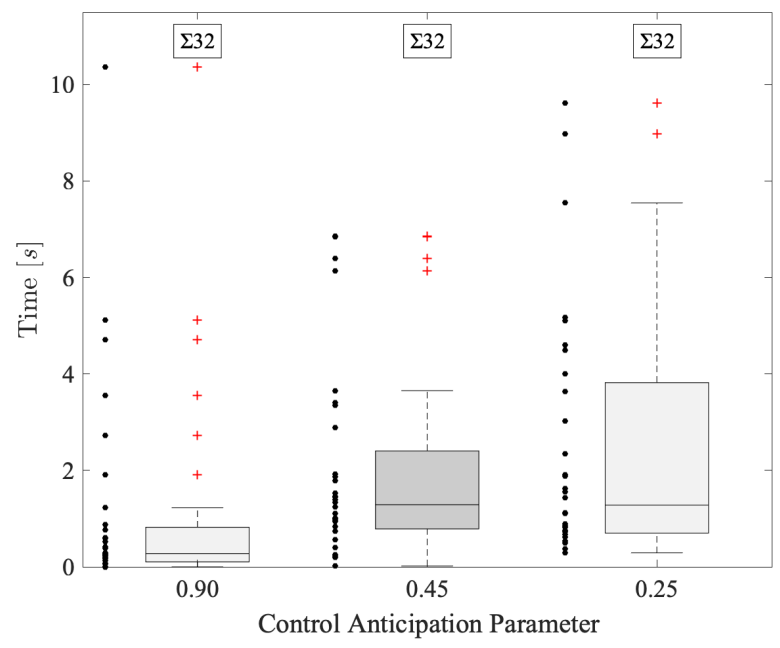

Fig. 14 Means and the $95 \%$ confidence limits of the combined flag sorted by CAP 


\section{Statistical Analysis of other dependent measures}

Performance of the test subjects using both models is shown in Figure 12. Since the data passed the normality and homogeneity of variance tests, a dependent t-test is performed to evaluate differences. Performance in the full model $(0.242 \pm$ 0.052) was significantly lower compared to the CAP reference model $(0.268 \pm 0.070)$ with $t(95)=3.387$ and $p<0.05$.

Control activity of test subjects using both models is shown in Figure 13. In contrast to the performance ratio, the control activity data did not pass the normality and homogeneity of variance tests. A Wilcoxon signed-rank test is therefore required to compare the results. This test showed a statistically significant lower control activity with the CAP reference model $(Z=-8.018$ and $p<0.00)$.

The combined flag duration is illustrated in Figure 14, as function of CAP levels. Mauchly's test $\chi^{2}(2)=6.225, p<$ 0.04 indicates that the assumption of sphericity is violated. Using a full-factor ANOVA and applying a Greenhouse-Geisser correction determined that the mean CAP differed significantly $\left(F_{1.684}=6.831, p<0.00\right)$. Post hoc tests using the Bonferroni correction (significance level of $p<0.02$ ) show that the CAP of $0.90(1.129 \pm 2.15)$ differs significantly from the CAP of $0.25(2.456 \pm 2.54) p<0.01$, but there are no significant differences between a CAP of 0.90 and $0.45(1.964 \pm 1.993)$ $p<0.03$ and a CAP of 0.45 and 0.25 with $p<0.49$. However, the difference between a CAP of 0.90 and 0.45 is close to the significance level of $p<0.02$.

\section{Discussion and conclusions}

The purpose of the simulator experiment was to identify whether pilots could notice the difference in handling qualities between the CAP reference logic and a full aircraft dynamic model including the controller.

The first hypothesis (H.1) is confirmed, since the performance of the pilots was lower for the full model compared to the CAP reference model. When actuator saturation is occurring, it is more difficult for pilots to maintain within the performance bandwidth.

H.2 is also confirmed. The control activity of the pilots was indeed higher for the full model compared to the CAP reference model. This can be explained by the higher control activity of pilots that occurs when the aircraft does not respond according to their expectations due to actuator saturation.

The full model is generally rated at a lower Cooper-Harper rating compared to the CAP reference model. This relates to the difference in response between the models in case of actuator saturation. In addition, some lag is present within the full model due to the actuator dynamics (requested deflection by the pilot is not instantly the actual deflection), whereas this lag is not present in the CAP reference model. At CAP values of 0.90 the mean difference between the experienced handling qualities was on average only 1 Cooper-Harper rating point, whereas this difference increased for lower CAP values with increased actuator saturation. This confirmed H.3, since the model mismatch was higher for CAP below the lower limits determined in offline simulation.

It was investigated how long the saturation time would be before the pilots experienced different handling qualities. However, the differences in Cooper-Harper rating are not directly related to the flag time. Additional runs would be required to investigate this in more detail. The lower limits obtained with the offline simulations (Figure 4) are updated with the acquired saturation time information as shown in Figure 15. The updated estimation includes the expected Cooper-Harper differences for specific CAP and damping settings for this control task. The updated simulation model can be used for different control tasks to investigate the limits of the full system.

\section{Experiment 2: Flight Test}

To validate the controller design and implementation, four flight tests were performed over the course of eight days. The flights all departed and terminated at Amsterdam Schiphol Airport (AMS) and were flown in the TMA Delta and above the North Sea, within the Dutch Airspace. The system is validated using the three initial flight tests, whereas the final flight was a demonstration flight.

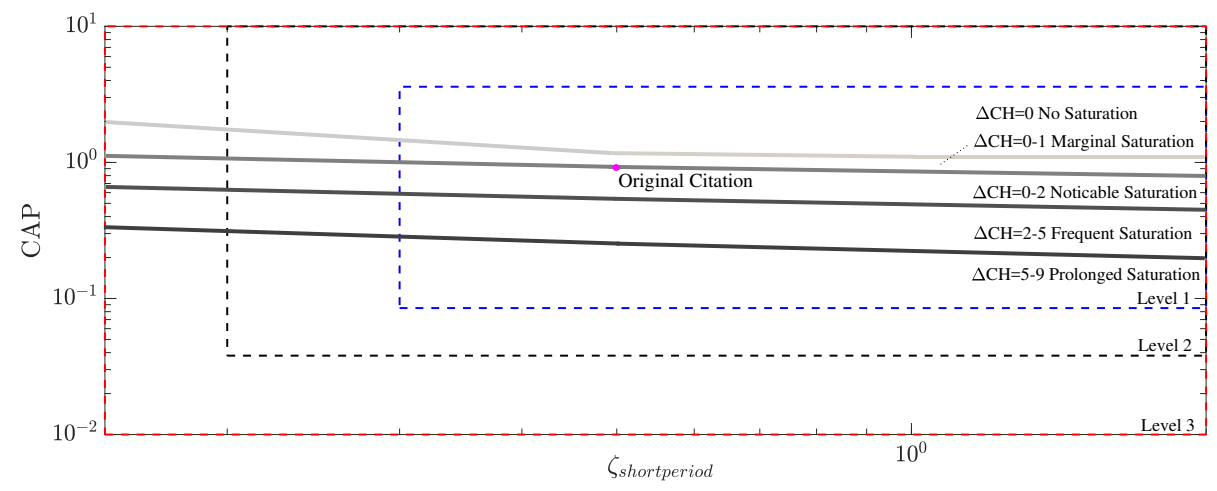

Fig. 15 Updated handling quality limitations using the combined flag saturation information 


\section{A. Method}

\section{Apparatus}

The Cessna Citation II business jet, co-owned by the Delft Aerospace Engineering faculty and the Netherlands Aerospace Centre, was used in the flight tests. The flight tests are performed in a clean configuration with spoilers, gear and flaps retracted. The angle of attack and sideslip are measured in clean air using a boom mounted in front of the aircraft. Activation of the automatic trim would disrupt the experimental control system, therefore the trim was deactivated during the experiment. The aircraft was controlled by the co-pilot using force input. The other pilot was the safety pilot during the experiment. The experimenter controlled the software and its variable stability settings via a wireless connection using a laptop in the cabin.

\section{Changes to controller}

Multiple changes were made to the controller with respect to the simulator version. The complete system dynamics were removed and changed to ensure that they only output the moment effectiveness matrix. All other outputs were received from sensors based on the aircraft itself. The requested deflection that comes from the INDI controller was linked to the autopilot servos from the existing aircraft fly-by-wire system [35].

New angular accelerometers were installed on the Cessna Citation II laboratory aircraft. These were an addition to the previously available angular accelerations which were established by differentiating the angular rates. Second order filters were introduced to these angular accelerations to remove noise whilst still maintaining the original signal without too much time delay. Especially the differentiated angular rate signals contained relatively high noise levels.

The controller required a conversion of the stick gains, since the stick on the aircraft was not identical to the electrohydraulic stick used in the fixed-base simulator. The stick on the aircraft converted the input from the pilots to a different scale, thus the gains were adapted to maintain the same scaling as in the simulator.

In comparison to the fixed initial conditions in the fixedbased simulator, the initial conditions depend on the actual flying conditions upon experiment initiation. The updated initial conditions were also used in the determination of the moment effectiveness matrix.

The fixed-base simulator experiment was based on the DASMAT model, which consisted of an inertia tensor based on the Cessna Citation I. Since the experiment is conducted in the Cessna Citation II, it was required to update this inertia tensor based on the newly developed model by Van den Hoek et al. [31]. The model mismatch between the DASMAT model and the new model was highest for the inertia around the y-axis $I_{y y}$, with a $35 \%$ mismatch. The $I_{x x}, I_{z z}$ and $I_{x z}$ showed a mismatch of $8 \%, 24 \%$ and $27 \%$ respectively.

\section{Procedure}

The experiments in pitch focused mainly on the longitudinal handling qualities, similar to the experiment in the simulator. In addition, a number of roll manoeuvres were performed to test the differences in roll time constants. To demonstrate the system potential to be used in pilot training, the effect of roll time constants, stick time delays and stick gains were investigated.

Two initial conditions were used during the different flight tests. Both conditions were at an altitude of FL130, but with a varying airspeed of 175 or 190 knots. To ensure safe execution of the experiment, a strict procedure was implemented during the experiments. First, the safety pilot flew to the requested altitude. Then he trimmed the aircraft for the desired airspeed in horizontal flight and disabled the auto trim. At the same time, the experimenter selected the desired parameter configuration in the DUECA software. Activation of the autopilot based on the controller software required activation of both the roll and pitch axes. This was achieved by a trigger of both the safety pilot and the experimenter, for each axis. Finally, the experimental control mode was initiated by the co-pilot, with "hands-off-stick" to prevent undesirable initial control inputs.

\section{Subjects and Instruction to Subjects}

To validate the controller by noticing the differences in handling qualities, pilots require experience on the Cessna Citation II laboratory aircraft. Three pilots have flown the aircraft in the experiments, being pilot $\mathrm{E}, \mathrm{F}$ and $\mathrm{G}$ (Table 2). In contrast to the fixed-based simulator experiments, the pilots did not assign Cooper-Harper ratings, due to the limited number of experimental runs.

Pilots received instructions for the different phases in flight. In accordance with the simulator task, the pilots were instructed to fly at $\pm 1000[\mathrm{feet} / \mathrm{min}]$ rate of climb during the test. However, the pilot was free to decide between ascent and descent given he remains below the FL135. This restriction was required, as a significant reduction of torque in the flyby-wire system is activated above FL135. During the lateral handling tests, the pilots were asked to assess differences between roll time constants. They decided on capturing a specific roll angle to investigate the performance of the system.

\section{B. Results}

\section{Longitudinal response}

The longitudinal tracking response of the controller can be seen in Figure 16, where the time on the horizontal axis represents the time passed after starting the controller in the aircraft. The pilot was requested to fly similar manoeuvres as in the simulator experiment. The controller was set with a CAP of 0.90 and a damping of 0.50 before 1160 seconds. After 1160 seconds the damping was changed to 0.15 , whereas the CAP remained the same (indicated by the vertical black dotted line). The reference signal could not be followed properly at some points in time, since the elevator servo was saturated. This is indicated by the current in the actuator servo which reaches 
the edges of the bandwidth (indicated by the horizontal black dotted lines). This is most visible at the 1260 seconds time point. In addition, some PIO was present for lower damping conditions. This is especially evident when comparing the pilot input to the reference signal between 1270 and 1300 seconds. The pilot also noticed this PIO himself when flying the aircraft. The lower damping was also identified by the pilot. The maximum $\Delta \delta_{e}$ during this run equalled $1.76 \cdot 10^{-3}[\mathrm{rad}]$ or 1.01 [deg], whereas the maximum requested deflection by the controller equalled $3.91 \cdot 10^{-2}[\mathrm{rad}]$ or $2.24[\mathrm{deg}]$.

The aircraft was properly trimmed before the run shown in Figure 16. The impact of improper trim is indicated in Figure 17. Some manoeuvres were performed before the start of this run which resulted in a mismatch between the trimmed and actual indicated airspeed of $\approx 10[\mathrm{~m} / \mathrm{s}]$. The effect is seen in the actuator servo current, where the initial value is not close to zero as indicated by the black line (in contrast to Figure 16). Additional actuator deflection was required to remain straight and steady flight at this higher speed, resulting in a nonzero current. The available bandwidth above the black line is smaller compared to the bandwidth below. This resulted in more actuator saturation and a lower maximum elevator deflection. Pilots noted this effect during flight, saying it was easier to control the aircraft in one direction compared to the other when the aircraft was not properly trimmed. A change of indicated airspeed of more than $5[\mathrm{~m} / \mathrm{s}]$ already resulted in this effect, according to the pilots. Next to this the pilots noted that the handling qualities of the aircraft were better when a lower pilot gain was used. This is confirmed in Figure 17, since higher stick deflections by the pilot result in more actuator saturation. The maximum $\Delta \delta_{e}$ during this
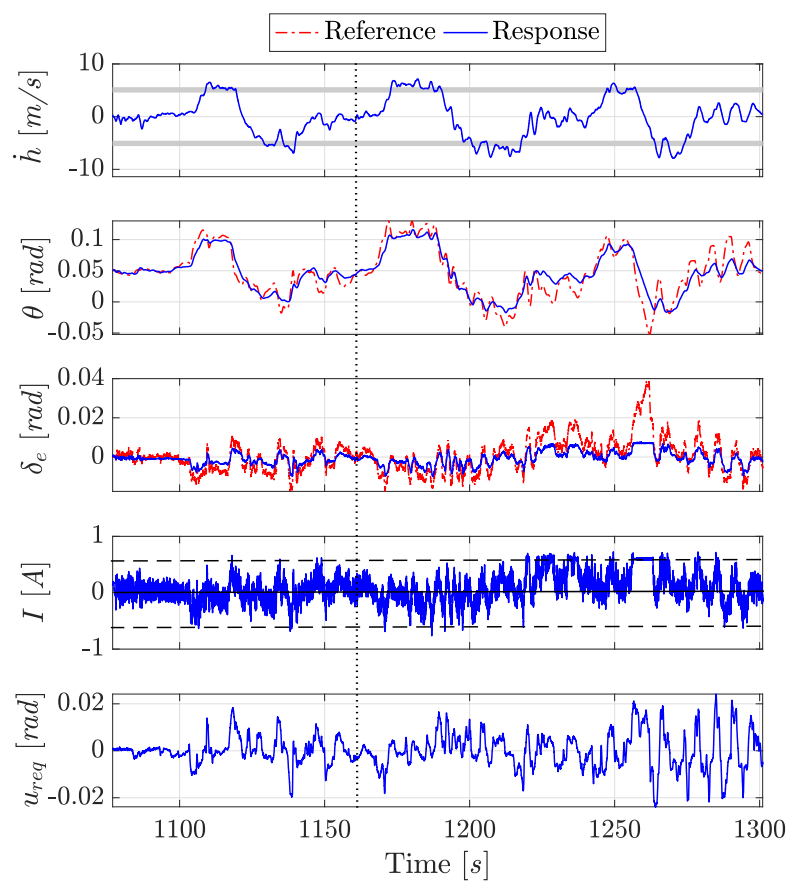

Fig. 16 Longitudinal tracking response $\left(V_{\text {ias }}=110[\mathrm{~m} / \mathrm{s}]\right.$ FL130) run equalled $1.61 \cdot 10^{-3}[\mathrm{rad}]$ or $0.917[\mathrm{deg}]$, whereas the maximum requested deflection by the controller equalled $1.43 \cdot 10^{-1}[\mathrm{rad}]$ or $8.08[\mathrm{deg}]$.

\section{Lateral response}

The lateral tracking response of the aircraft during one of the runs is given in Figure 18. The fly-by-wire system shows similar limitations compared to the longitudinal response. The controller has proper tracking performance during the turn between 1800 and 1915 seconds. However, saturation also occurs in the aileron actuator servo if the pilot gives a larger input to the stick. This initially occurs at 1940 seconds, but is more visible from 1970 to 2010 seconds. The servo current reaches the edges of the bandwidth which results in poor tracking performance. This poor tracking performance results in PIO in the roll axis. The maximum $\Delta \delta_{a}$ during this run equalled $9.81 \cdot 10^{-2}[\mathrm{rad}]$ or $5.62[\mathrm{deg}]$, whereas the maximum requested deflection by the controller equalled $2.59 \cdot 10^{-1}[\mathrm{rad}]$ or $14.9[\mathrm{deg}]$.

\section{Discussion and conclusions}

Four flight tests were conducted to validate the variable stability controller. During the third and fourth flight test, the pilots noted that response of the controller and the aircraft was similar to the experiment in the fixed-base simulator. This is confirmed by the comparison between the reference dynamics and the actual response of the aircraft. Due to time and cost limitations, the simulator experiment could not be exactly reproduced in the real flight test. However, pilots commented that they could notice differences between different damping
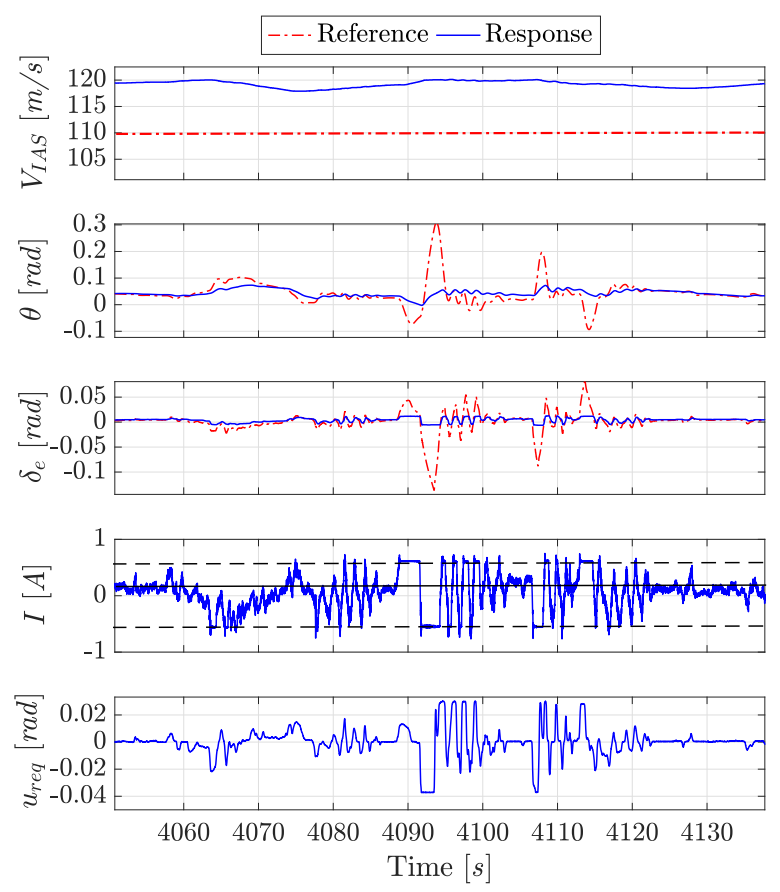

Fig. 17 Longitudinal tracking response $\left(V_{\text {ias }}=120[\mathrm{~m} / \mathrm{s}]\right.$ FL130) 

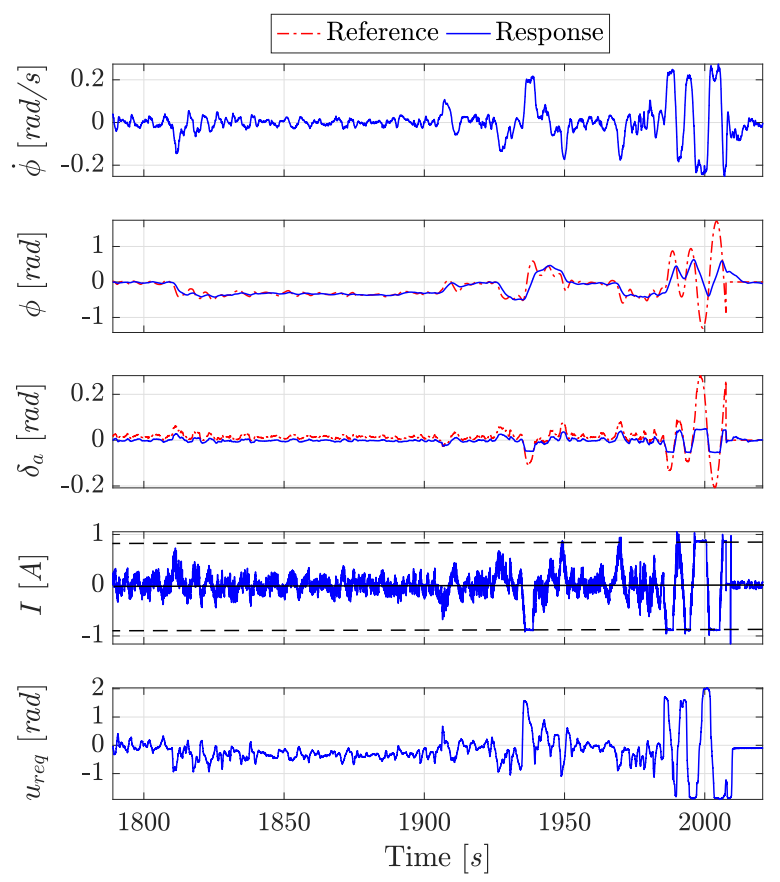

Fig. 18 Lateral tracking response $\left(V_{\text {ias }}=110[\mathrm{~m} / \mathrm{s}]\right.$ FL130)

settings, similar to the experiment in the fixed-base simulator.

The system limitations were identified by pushing the system in roll and pitch whilst deviating from the initial trim condition. When the aircraft was within the performance bandwidth of the actuator servos, the INDI controller was able to track the reference signal quite well. It is expected this can be further improved by implementing the aerodynamic model from the Cessna Citation II by Van den Hoek et al. [31], since the controller currently uses the aerodynamic model from the Citation I. However, actuator saturation is considered to be the most limiting factor, since tracking performance significantly decreased when saturation occurred. The requested deflection by the controller was in extreme cases both for the pitch as roll axis higher than the actual saturated deflection, by a factor of 8-10 in pitch and 3-4 in roll respectively. This is considered to be the most limiting factor of the variable stability system performance.

The most obvious solution is to replace the current fly-bywire system with a more powerful system. This is however an expensive hardware change which requires recertification. A possible improvement of the current system can be achieved by adding control of the elevator trim in the control laws. Future research should investigate whether a lower hinge moment can be achieved using the auto trim without interfering with the actual response of the elevator. This could increase the performance band of the system in longitudinal motion but would require hardware changes to the aircraft. Another solution would be to keep the auto trim activated and use the software model to estimate when the auto trim is going to occur. This could be used to minimise the interference of the auto trim on the actuator deflection. However, the expected non-linearities during flight reduce the accuracy of auto trim function predictions. This method is therefore considered a complex alternative.

\section{Discussion and Recommendations}

The purpose of this paper was twofold. First it was investigated if an INDI controller can be used to create a variable stability in-flight simulator. Second, the limitations of the system were researched. The controller was tested in offline simulations, during a fixed-base simulator experiment and in actual flight tests.

\section{A. Handling qualities}

The INDI technique is able to modify the handling qualities of an aircraft in a variable stability system. Offline simulations showed that the controller manipulates the response of the actuators to ensure tracking performance of the CAP reference model. The flight condition that provides the best performance for the Cessna Citation II business jet was determined to be at $4000[\mathrm{~m}]$ altitude with a true airspeed of $110[\mathrm{~m} / \mathrm{s}] . \mathrm{PCH}$ improved the controller performance by slightly modifying the CAP reference signal when there was no actuator saturation present. However, the hedging signal adapted the CAP reference logic more significantly when the actuators were saturating. This implies that the handling qualities change when PCH is enabled, which is undesired behaviour.

An experiment was performed in a fixed-base simulator to determine if pilots could notice the difference between the CAP reference model and a full model including the aircraft and actuator dynamics. The control activity of the pilots was higher for the full model compared to the CAP reference model, and the performance of the pilots was lower for the full model. This can both be explained by pilots who are not experiencing the expected response of the aircraft when actuators are saturating.

Pilots rated the full model on average two Cooper-Harper points lower compared to the reference dynamics model. It was hypothesised that this difference would occur due to actuator saturation. This was confirmed by the differences of rating for the different CAP conditions. The CAP of 0.90 was rated at a mean Cooper-Harper difference of one, which differed statistically from the CAP of 0.45 and 0.25 with a mean CooperHarper difference of two. The CAP of 0.90 also showed a shorter maximum saturation time compared to the CAP of 0.25 , whereas the difference between CAP 0.90 and 0.45 was close to the significance level. This confirmed the initial estimation of the system limitations. The actuator saturation data was compared with the Cooper-Harper differences between the CAP reference and the full model. The saturation time was used to update the model that estimates the handling quality limitations.

Flight tests were performed to validate the performance of the controller. Pilots noted that they experienced the changes in CAP and damping similar to the fixed-base simulator experiment. However, they also experienced some limitations to the system. 


\section{B. System limitations}

Two factors were found to be limiting for the variable stability in-flight simulator using the INDI technique. The first factor was the accuracy of the moment effectiveness parameter. This parameter depends on the accuracy of both the aerodynamic coefficients and the aircraft inertia. During the offline simulations and the fixed-base experiment the controller showed proper tracking performance. Even in the flight tests, when additional non-linearities and disturbances are present, the INDI controller could track the reference model properly. This can be further improved, however, by updating the aerodynamic coefficients to the newer Cessna Citation II model.

The second and most limiting factor was found to be the actuator saturation. The tracking performance of the controller decreased significantly when actuator saturation occurred. Saturation occurred either when the input of the pilot was too high, or when the aircraft deviated too much from its initial trim condition. The maximum deflection of the elevator was approximately $1[\mathrm{deg}]$, whereas the maximum deflection of the ailerons was around $6[\mathrm{deg}]$. These limited deflections arose from the constraints of the fly-by-wire system. Future research should investigate whether a lower hinge moment can be achieved using the auto trim, without interfering with the actual position of the elevator. This could increase the performance band of the system in longitudinal motion but would also require hardware changes to the aircraft.

\section{Conclusions}

The purpose of this research was to investigate whether the Cessna Citation II could be converted in a variable stability system, using incremental non-linear dynamic inversion. Offline simulations show that the INDI control method can be implemented to achieve a variable stability platform. PseudoControl Hedging can be used to increase the performance of the controller, but it also directly influences the handling qualities.

A fixed-base experiment showed that pilots could feel differences between the reference dynamics and a full model including actuators and the INDI controller. Pilots rate the full model on average two Cooper-Harper points lower compared to the reference dynamics model. The experienced model mismatch was higher for CAPs below the lower system limits as determined in the offline simulation, and actuator saturation increased for lower CAPs.

Four flight tests were conducted to validate the variable stability controller. Pilots commented that they could notice differences between different damping settings, similar to the experiment in the fixed-base simulator.

The system limitations were identified by pushing the system in roll and pitch whilst deviating from the initial trim condition. Especially the longitudinal motion was considered to be limited due to actuator saturation.

Future work will focus on increasing the performance envelope around the initial condition.

\section{Acknowledgements}

The authors would like to thank the involved staff from the Delft University of Technology: Hans Mulder, Alexander in 't Veld, Menno Klaassen, Ferdinand Postema and Gijsbert den Toom. Their support, guidance and additional time during the project and especially the flight tests was highly appreciated. Finally, the authors would like to thank all the pilots that participated in the experiments.

\section{References}

[1] Weingarten, N. C., "History of In-Flight Simulation at General Dynamics," Journal of Aircraft, Vol. 42, No. 2, 2005, pp. 290-298. doi:10.2514/1.4663.

[2] Hamel, P., In-Flight Simulators and Fly-by-Wire / Light Demonstrators, Springer Nature, 2017.

[3] Charrier, J., and Kulshreshtha, A., "Electric Actuation for Flight and Engine Control; Evolution and Current Trend," 45th AIAA Aerospace Sciences Meeting and Exhibit, Aerospace Sciences Meetings, American Institute of Aeronautics and Astronautics, 2007. doi:10.2514/6.2007-1391, URL https: //doi.org/10.2514/6.2007-1391.

[4] Mirza, A., Van Paassen, M. M., and Mulder, M., "Simulator Evaluation of a Medium-Cost Variable Stability System for a Business Jet," Proceedings of the AIAA Guidance, Navigation, and Control Conference, 2019.

[5] Armstrong, E. S., A Design System for Linear Multivariable Control, New York: Marcel Dekker, 1980.

[6] O’Brien, M. J., and Broussard, J. R., “"Feedforward Control to Track the Output of a Forced Model." Decision and Control including the 17th Symposium on Adaptive Processes," IEEE Conference on. IEEE, 1979, pp. 835-842.

[7] Kreindler, E., and Rothschild, D., "Model-Following in LinearQuadratic Optimization," American Institute of Aeronautics and Astronautics Journal, Vol. 14, no. 7, 1976, pp. 835-842.

[8] Slotine, J. J. E., and Li, W., Applied Nonlinear Control, Englewood Cliffs, N.J.: Prentice Hall, 1991.

[9] Ko, J., Lee, H., and Lee, J., "In Flight Simulation for Flight Control Law Evaluation of Fly-by-Wire Aircraft," Agency for Defense Development, Daejeon, Republic of Korea, 2004.

[10] Ko, J., and Park, S., "Variable stability system control law development for in-flight simulation of pitch/roll/yaw rate and normal load," International Journal of Aeronautical and Space Sciences, Vol. 15, No. 4, 2014, pp. 412-418. doi:10.5139/ IJASS.2014.15.4.412.

[11] Miller, C., "Nonlinear Dynamic Inversion Baseline Control Law: Architecture and Performance Predictions," AIAA Guidance, Navigation, and Control Conference, Guidance, Navigation, and Control and Co-located Conferences, American Institute of Aeronautics and Astronautics, 2011. doi:10.2514/6.2011-6467, URL https: //doi .org/10.2514/6.2011-6467.

[12] Smith, P., "A Simplified Approach to Non-linear Dynamic Inversion Based Flight Control," 23rd Atmospheric Flight Mechanics Conference, 1998. doi:10.2514/6.1998-4461, URL http://arc .aiaa.org/doi/10.2514/6.1998-4461. 
[13] Germann, K. P., T-6A Texan II In-Flight Simulation and Variable Stability System Design, Mississippi State University, 2006.

[14] Grondman, F., Looye, G., Kuchar, R. O., Chu, Q. P., and Van Kampen, E., "Design and Flight Testing of Incremental Nonlinear Dynamic Inversion-based Control Laws for a Passenger Aircraft," 2018 AIAA Guidance, Navigation, and Control Conference, AIAA SciTech Forum, American Institute of Aeronautics and Astronautics, 2018. doi:10.2514/6.2018-0385, URL https://doi.org/10.2514/6.2018-0385.

[15] Lubbers, B., A Model of the Experimental Fly-By-Wire Flight Control System for the PH-LAB, Delft University of Technology (unpublished master thesis), 2009.

[16] Nelson, R. C., Flight Stability and Automatic Control, McGrawHill Education, 1998.

[17] Bryson, A. E., and Ho, Y. C., Applied Optimal Control, Hemisphere, Washington D.C., 1975.

[18] Ko, J., and Park, S., "Design of a Variable Stability Flight Control System," KSAS International Journal, Vol. 9, No. 1, 2008.

[19] Bacon, B., and Ostroff, A., "Reconfigurable Flight Control using Non-linear Dynamic Inversion with a special Accelerometer Implementation," AIAA Guidance, Navigation, and Control Conference and Exhibit, 2000. doi:10.2514/6.2000-4565, URL http://arc.aiaa.org/doi/10.2514/6.2000-4565.

[20] Cox, T., and Cotting, M., "A Generic Inner-Loop Control Law Structure for Six-Degree-of-Freedom Conceptual Aircraft Design," 43rd AIAA Aerospace Sciences Meeting and Exhibit, Aerospace Sciences Meetings, American Institute of Aeronautics and Astronautics, 2005. doi:10.2514/6.2005-31, URL https://doi .org/10.2514/6.2005-31.

[21] Smeur, E. J. J., Chu, Q. P., and de Croon, G. C. H. E., “Adaptive Incremental Nonlinear Dynamic Inversion for Attitude Control of Micro Air Vehicles," Journal of Guidance, Control, and Dynamics, Vol. 39, No. 3, 2016, pp. 450-461. doi:10.2514/1. G001490.

[22] Smeur, E. J. J., de Croon, G. C. H. E., and Chu, Q. P., "Cascaded Incremental Nonlinear Dynamic Inversion Control for MAV Disturbance Rejection," Control Engineering Practice, 2017, pp. 1-12.

[23] Cooper, G., and Harper, R., "Handling Qualities and Pilot Evaluation," Journal of Guidance, Control, and Dynamics, Vol. 9, No. 5, 1986, pp. 515-529. doi:10.2514/3.20142.

[24] Bihrle, W., "A Handling Qualities Theory for Precise Flight Path Control," Tech. rep., Air Force Flight Dynamics Laboratory, New York, 1966.

[25] Standard, MIL-STD-1797A Requirements for Flying Qualities of Piloted Aircraft, Department of Defense, 1990.

[26] Bischoff, D. E., "The Control Anticipation Parameter for Augmented Aircraft," Tech. rep., Naval Air Development Center, Warminster, 1981.

[27] Di Franco, D. A., "In-flight Investigation of the Effects of HigherOrder Control System Dynamics on Longitudinal Handling Qualities," Tech. rep., Cornel Aeronautic Lab, Buffalo NY, 1968. doi:10.1002/nav.3800080206.
[28] Hodgkinson, J., LaManna, W. J., and Heyde, J. L., "Handling Qualities of Aircraft with Stability and Control Augmentation Systems - A Fundamental Approach,” Vol. 80, 1976, pp. 75-81.

[29] Morelli, E. A., Identification of Low Order System Models From Flight Equivalent Test Data, NASA Langley Research Center, 2000.

[30] Borst, C., CITAST: Citation Analysis and Simulation Toolkit, Delft University of Technology, Delft, 2004.

[31] Van den Hoek, M. A., de Visser, C. C., and Pool, D. M., "Identification of a Cessna Citation II Model Based on Flight Test Data," Advances in Aerospace Guidance, Navigation and Control, 2018, pp. 259-277.

[32] Johnson, E. N., and Calise, A. J., "Pseudo-Control Hedging : a New Method for Adaptive Control,", 2000.

[33] Looye, G., Thümmel, M., Kurze, M., Otter, M., and Bals, J., "Non-linear Inverse Models for Control," Proceedings of the 4th Modelica Conference, 2005, pp. 267-279.

[34] van Paassen, M. M., Stroosma, O., and Delatour, J., "DUECA - Data-driven Activation in Distributed Real-Time Computation," Modeling and Simulation Technologies Conference, Guidance, Navigation, and Control and Co-located Conferences, American Institute of Aeronautics and Astronautics, 2000. doi: 10.2514/6.2000-4503, URL https://doi .org/10.2514/6. $2000-4503$.

[35] Zaal, P., Pool, D. M., in 't Veld, A. C., Postema, F., Mulder, M., van Paassen, M. M., and Mulder, J. A., "Design and Certification of a Fly-by-Wire System with Minimal Impact on the Original Flight Controls," AIAA Guidance, Navigation, and Control Conference, 2009. doi:10.2514/6.2009-5985, URL http://arc . aiaa.org/doi/10.2514/6.2009-5985. 


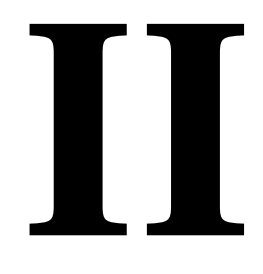

\section{Preliminary Thesis \\ Previously graded under AE4020}





\section{1}

\section{Introduction}

A variable stability in-flight simulator is an aircraft which has the capabilities to change the stability coefficients in flight, often without changing the physical properties of the aircraft. It allows variations of response characteristics and handling qualities of an aircraft, which can be used for many different purposes like pilot training, control logic development and handling quality research. [1]

Variable stability systems have been designed from the 1940's and onward. In the early years, their purpose was to design control logic for different flight situations like landing on an aircraft carrier. In the 70's and 80's, focus moved from specific flight situations or aircraft configurations to testing conceptual control logic of new aircraft before they were actually built. More recent developments show the possibility for the in-flight simulator to follow the dynamics of different aircraft. An historical overview of variable stability systems is given in section 2.1. [2]

The Control and Simulation department of Delft University of Technology would like to investigate the possibility of transforming its Cessna Citation II aircraft into a variable stability in-flight simulator. Some of many applications are listed below:

- Pilot training: Pilots can be trained using a variable stability in-flight simulator. For example, the impact of changes in aerodynamic coefficients or centre of gravity position can be modelled. In this way a pilot can experience these kind of situations without actually having to modify the aircraft physically.

- Lower cost: If other aircraft or dynamics can be modelled, this can be used in the training program of pilots. Often they have to fly with the specific aircraft itself, which might be much more expensive. One can imagine that the operational cost of an Airbus A320 might be a lot higher compared to the Cessna Citation II.

- Handling qualities testing: The handling qualities of new aircraft can be tested before the aircraft is even flown. The in-flight simulation would follow the theoretical response from the new aircraft, so pilots can experience its handling and performance.

- Basis for other research: Using a flexible method to achieve variable stability, it can easily be used or implemented by other students or researchers, without requiring much in-depth knowledge of the system.

Previous research at the Control and Simulation department regarding variable stability was performed by Mirza et al. [3]. He focused mainly on how feedback on specific aircraft states would modify the Control Anticipation Parameter (CAP). Next to this, he tested if pilots could detect changes in CAP. His research is described in more detail in the chapter 2.

Any variable stability method would use the fly-by-wire system of the Cessna Citation II to achieve its performance. That is why its limitations have to be investigated. Next to this, it is expected that some limits exists regarding the variable stability that can be achieved using the Cessna Citation II. Both topics are investigated in more detail in this preliminary thesis. 


\subsection{Research objective}

The research objective of this thesis is formulated as follows: "The research objective is to design a variable stability control system for the Cessna Citation II by determining the variable stability control limits, developing variable stability control logic and testing this logic."

This research objective can be broken down in multiple sub-goals, which are easier to handle. The sub-goals of this thesis are defined as follows:

1. Perform a literature study to acquire knowledge about the history of variable stability systems and the different methods that can be applied to achieve variable stability.

2. Gather flight data that can be used to determine the variable stability control limits.

3. Determining the variable stability control limits by investigating the fly-by-wire limitations and the different variable stability method limitations.

4. Developing a tool to asses the handling qualities (CAP) of the variable stability system.

5. Develop the variable stability control logic by selecting the most suitable methods found from literature and the preliminary research. Compare the performance and limitations of different controllers. Use the controllers to evaluate the CAP limitations of the system.

6. Verify and validate the model by testing the variable stability control logic in offline simulations, in SIMONA and during flight tests.

Next to these sub-goals, it is important to clearly document all development steps and the final product properly, so it can be used by the Control and Simulation department in other (research) projects.

\subsection{Research question}

The main research question for this thesis is formulated as follows: Is it possible to design a model-following variable stability system for the Cessna Citation II?

This research question can be split in several sub-questions. They are defined as follows:

1. What are the different variable stability control methods and their limitations?

2. What are the fly-by-wire control limits of the Cessna Citation II?

3. Which variable stability control method is best applicable to the Cessna Citation II and the requirements from the department?

The research question and its sub-questions will be answered by completing the sub-goals as described earlier. The research objective and research questions lead to the research framework as presented in Figure 1.1.

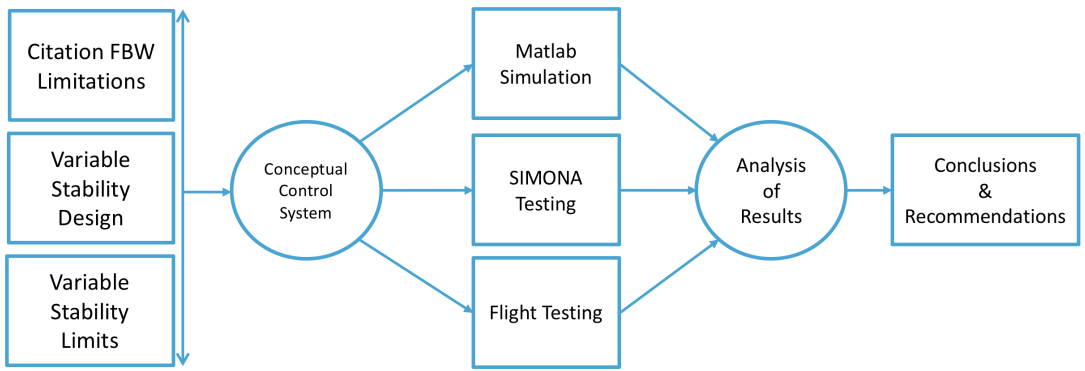

Figure 1.1: Research framework for the thesis project 


\subsection{Methodology and report structure}

As the main research objective is to design a variable stability control system, verify the functionality and validate its results, the steps that must be taken correspond to those of a control design framework. The methodology to achieve the main research objective can be structured according to the sub-goals presented before. The result of this can be found in Figure 1.2. The different steps are explained in more detail.

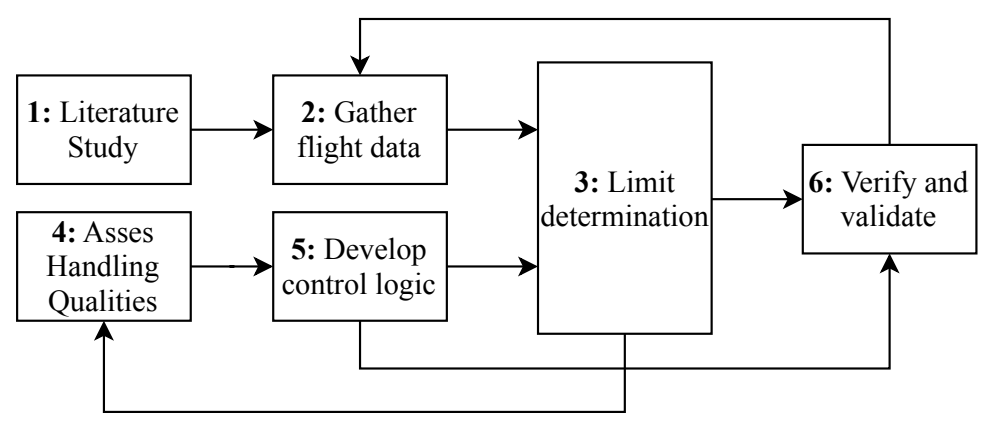

Figure 1.2: The structure of the proposed thesis research methodology

Step 1 - Literature study: This step is required to acquire knowledge about variable stability in-flight simulators and their different methods. It also gives an overview of applications of different methods that already exist. Some history, variable stability methods, handling quality determination methods and simulation models are presented in Chapter 2.

Step 2 - Gather flight data: After acquiring sufficient knowledge concerning the variable stability systems and methodology, some flight data has to be gathered to be able to proceed with the limit determination. Sufficient flight test data is already available due to some fly-by-wire flight tests in collaboration with Deutsches Zentrum für Luft- und Raumfahrt (DLR), but it has to be converted to usable data since the control surface logging is not incorporated in the flight data logging system. Next to this, the actuator model can be validated and adapted where necessary using the flight data. This process is described in Chapter 3 .

Step 3 - Limit determination: Using the processed flight data, the limits from the fly-by-wire system can be researched. Next to this, the limitations arising form the different variable stability methods can be identified. This combined information would lead to a beneficial operating point in the flight envelope of the Cessna Citation II. These system limitations are discussed in Chapter 4.

Step 4 - Asses handling qualities: A tool has to be developed to asses the handling qualities. The background information for this assessment can be found in Section 2.3. An initial implementation and test of this tool is found in Chapter 5. When the control logic is developed, it can be used for determining the limits of the variable stability system. Next to this, a sanity check regarding the rate of climb with the fly-by-wire system is also given in Chapter 5.

Step 5 - Develop control logic: Using the information from the literature study and the experience at the Control and Simulation department, control logic of a variable stability method can be designed. It is preferred to test at least two methods and compare their performance and robustness. This is not part of this preliminary thesis.

Step 6 - Verify and validate: The designed control logic has to be implemented and tested in offline simulations, in SIMONA and finally in the Cessna Citation II itself. During this part of the thesis new data will be gathered from those tests and simulations, which will again be used to evaluate the limits of the system and even improve the design in an iterative process. This is also not yet part of this preliminary thesis. 



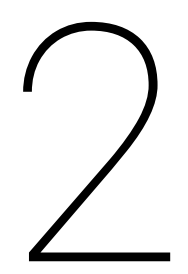

\section{Literature study}

This chapter contains the literature study of this research, which will be required for the further development of a variable stability in-flight simulator. First, a historical overview regarding the development of variable stability in-flight simulators is given. Secondly, different methods are discussed to achieve a variable stability. Thirdly, it is briefly discussed how to determine the handling qualities of an aircraft. Finally, before initial research can start, some simulation models are discussed that can be used for this research.

\subsection{Historical overview}

The purpose of this section is to give an overview of the extensive history within the variable stability system design. Many developments have taken place regarding variable stability systems, but just a brief portion is presented here. Most information in this chapter is a summary from work by P. Hamel in 2017, who wrote a comprehensive overview of this topic.[2] The main developments have taken place in two organisations, being Calspan Corporation (CAL) and National Aeronautics and Space Administration (NASA) (whilst still named National Advisory Committee for Aeronautics (NACA)). These developments will be discussed below.

\subsubsection{Calspan development}

The first CAL project concerning variable stability was the design of split rudder for the Vought F4U-5 VS in the 1940's as can be seen in Figure 2.1a. The purpose of this project was to improve the lateral stability by feeding back the sideslip angle and the yaw rate. Next to this, the flaps were modified to be controlled independently, which could artificially influence the roll and yaw rates and the angles of sideslip. This project lead to the new lateral-directional flying quality guidelines for the United States (US) Navy.

During the 1950's some similar projects followed, where different aircraft were modified to artificially stabilise the yaw, roll and pitch axes. Often this meant introducing an extra control surface or modifying existing parts of the aircraft. At this time it was also realised that more safety measures were required, so the safety pilot was introduced. The safety pilot is a second pilot that can take over control of the original aircraft logic when the to be tested system fails or proves to be unsafe or unstable.

The next main milestone concerned the development of optimal flying qualities criteria for the longitudinal motion. This development started by modifying the Lockheed F-94A (Figure 2.1b), with an extra rudder control surface, into the EF-94A. It was the first aircraft that was capable of evaluating control laws for aircraft that were still in the development phase. One example is the tests of the longitudinal logic for the Convair XB-58 supersonic jet. Even though the EF-94A could be used for many applications, it was limited in its space for analog computer systems and flight data recordings. Finally it was replaced by the Lockheed NT-33A in 1957 (Figure 2.2a). 


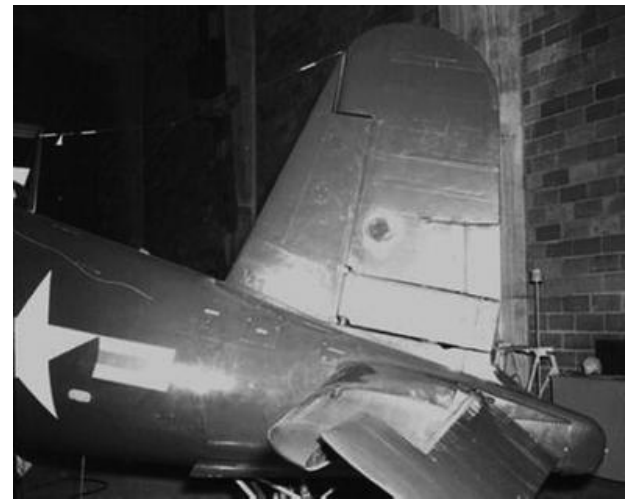

(a) Split rudder for the Vought F4U-5 VS

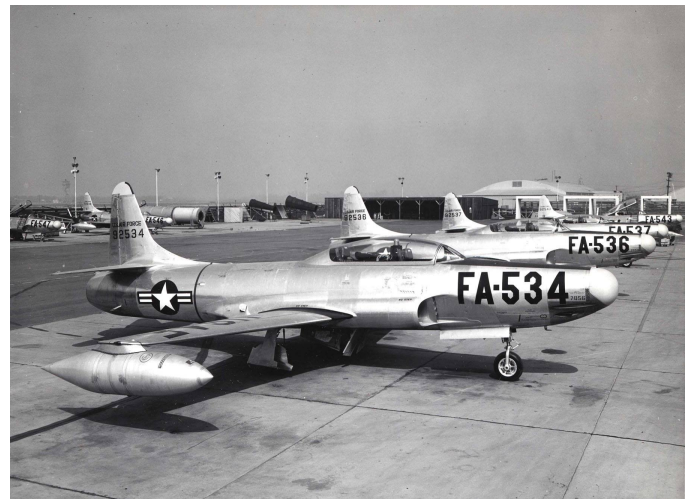

(b) Lockheed F-94A

Figure 2.1: CAL variable stability system development aircraft [2]

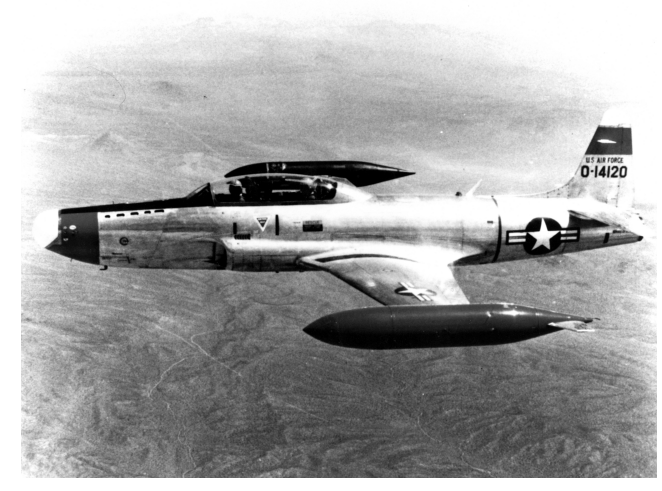

(a) Lockheed NT-33A

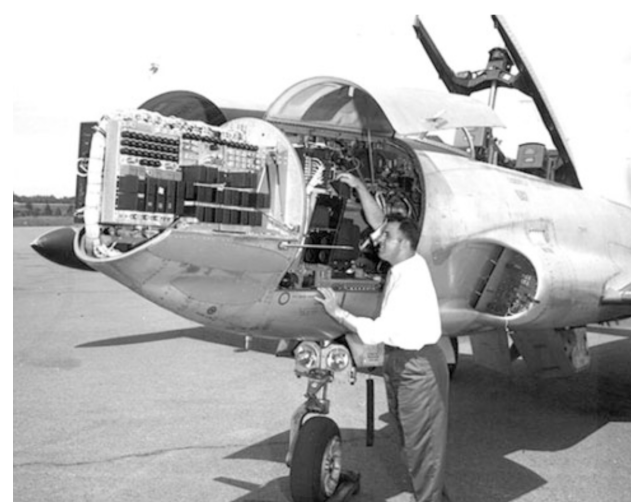

(b) Lockheed NT-33A with EF-94A nose

Figure 2.2: Lockheed NT-33A variable stability system development [2]

The Lockheed T-33A fuselage was adapted with the nose of the EF-94A (Figure 2.2b) to create enough space for the required analog simulation equipment, turning the aircraft into the NT-33A. Initially it was adapted to simulate the steep decent of the X-15 hypersonic aircraft, so it could be used by the pilots for training. The NT-33A was one of the most successful in-flight simulators worldwide. It supported the development of many different projects, like the A-9, A-10, F-15, F-16, F-17, F-18 and the F-22. It also provided support on international projects like the TSR2 and the JAS Gripen. A total of 8000 flight hours were flown with the NT-33A divided over 5200 different flights until 1997. Many of these hours were spent on training of test pilots. Robert Harper, famously known from the Cooper-Harper scale [4], developed many of these training programs for the US Airforce.

The next major development from CAL was the Convair NC-131H Total In-Flight Simulator (TIFS) in the 1970's. This TIFS has played a major role in the transport aircraft sector. CAL heavily modified this aircraft, by changing the engines, adding side-force generators and even adding an extra cockpit for the pilots who were flying the simulation (Figure 2.3a). The original cockpit was maintained as can be seen in Figure 2.3b. From there, the safety pilots could always intervene during a simulation.

Due to these changes to the original configuration, this aircraft was the first to manipulate the aircraft dynamics in all six degrees of freedom (pitch, roll, yaw, horizontal, vertical, lateral). The NC-131H was also the first aircraft that used the explicit model-following technique, in contrary to the previously used implicit modelfollowing style. These models will be explained in more detail in Section 2.2. The TIFS made its maiden flight in 1970 and has been used until 2008, with almost 40 years in service and 2500 test flights. Many projects were researched before their first actual flight, like the X-29, X-40, B-2, YF 23, C-5 and C-17 as well as civil projects like the Boeing 777 and the MD-12X. 


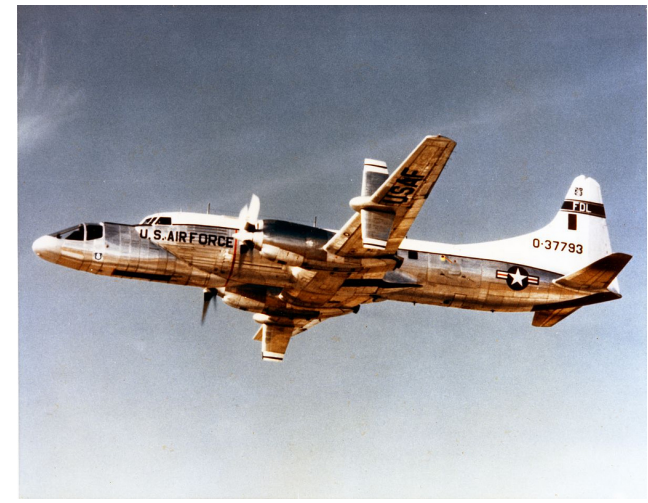

(a) Original TIFS configuration

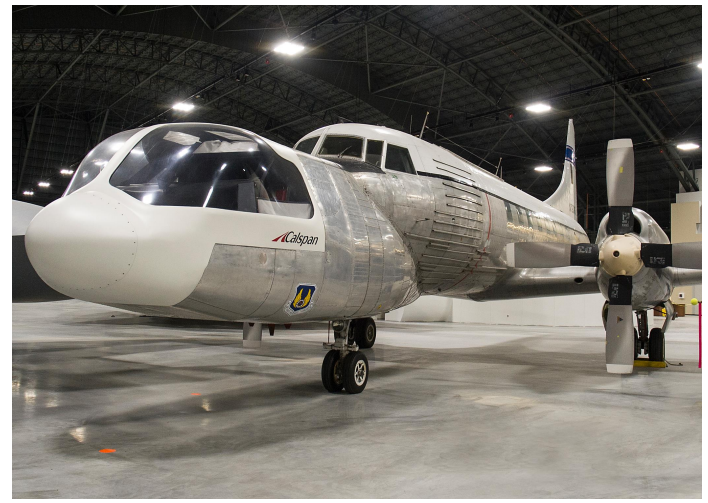

(b) Final configuration in Airforce museum

Figure 2.3: Convair NC-131H TIFS variable stability system development [2]

Increasing demand for pilot training surged the development of the Learjet 25D in the 1980's and the Learjet $25 \mathrm{~B}$ in the 1990's (Figure 2.4a). The early versions included pre-programmed variable stability conditions to simulate 128 aircraft conditions using response feedback. In the 90's both aircraft were updated to benefit from explicit modelling control logic, which allowed in-flight simulation of pitch, roll and yaw. In 2007 and 2014, newer Learjets were added to CAL's fleet. This was mainly to meet the increased demand in flight test programs for both civil and military aircraft projects and their components.

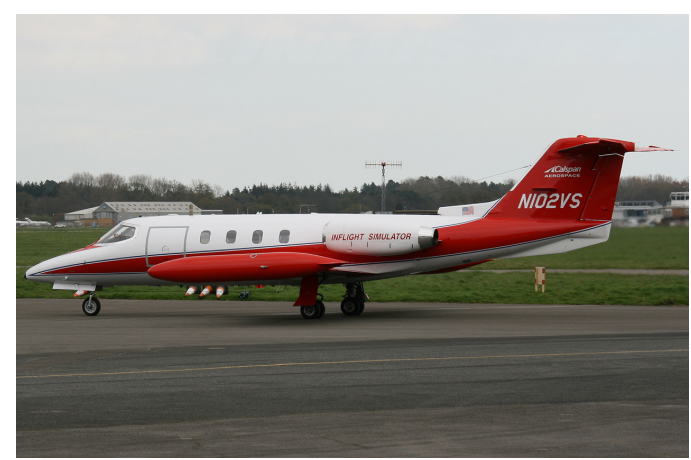

(a) CAL Learjet 25B

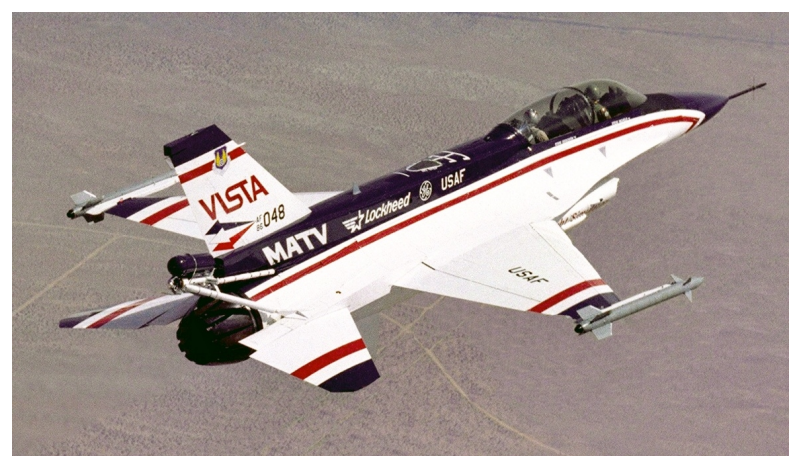

(b) General Dynamics NF-16D VISTA

Figure 2.4: CAL modern variable stability systems [2]

The General Dynamics NF-16D Variable Stability In-Flight Simulator Test Aircraft (VISTA) (Figure 2.4b) is an aircraft from the US Airforce, where CAL was subcontracted to develop the variable stability systems. Its variable stability characteristics where designed so pilots could experience different kind of training situations. A thrust vectoring system was installed to increase the variable stability capabilities. More recently it was used for testing the response of pilots to simulated damage or out-of-control situations. It has also been modified for testing of Unmanned Aerial Vehicle (UAV) flight control software and is still used for research at the time of this writing.

\subsubsection{NASA development}

NASA Ames Research Center also played a pioneering role in the development of in-flight simulators. The first basic idea of variable stability already existed back in 1948, when William Kaufmann saw the need for the development of such a system. [2] For the determination of wing dihedral, normally three different prototypes would be built to test which configuration would be favourable. One can imagine the high cost for this kind of development, which is why William Kaufmann designed a concept for artificially influencing the rolling moment due to side slip in flight using tools based on control technology.

Work in the 1940's and 1950's at NASA was mostly focused on variable stability in the yaw direction, mainly to investigate the damping motion of the Dutch Roll eigenmode (examples being the Lockheed T- 02 and the 
North American F-86A VS). In the following years, focus at NASA turned mostly towards the development of longitudinal handling qualities using variable stability systems. One interesting example is the North American YF-86D, which was designed with adaptable direct feedback in the longitudinal mode. With this vehicle, unstable flight configurations were flown for the first time. These investigations were later used in the development for fly-by-wire systems for unstable aircraft.

One of the main developments at NASA focused on the training of the pilots that were going to fly the space shuttle vehicle. The space shuttle classified as an extremely sluggish vehicle, which meant that a lot of training was required to control it properly. NASA acknowledged that the variable stability technology could be very useful in the training of these pilots. This lead to the development of the Grumman C-11A Shuttle Training Aircraft (STA) as can be seen in Figure 2.5a. During the space shuttle program, four of these aircraft were manufactured over a time period of more than 30 years. One can see the cockpit in space shuttle configuration in Figure 2.5b on the left side, where the safety pilot could always intervene if necessary. Astronauts in training had to fly over 500 descents in the STA before they were allowed to fly the space shuttle for the first time. With the end of the space shuttle project, the aircraft were also retired in 2011.

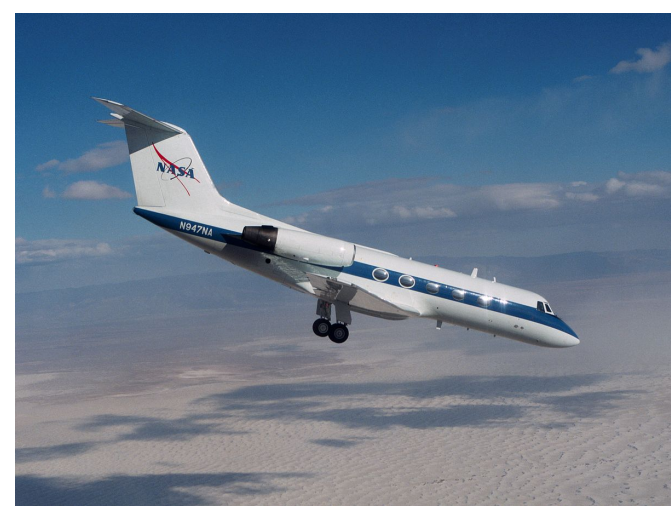

(a) STA in space shuttle decent simulation

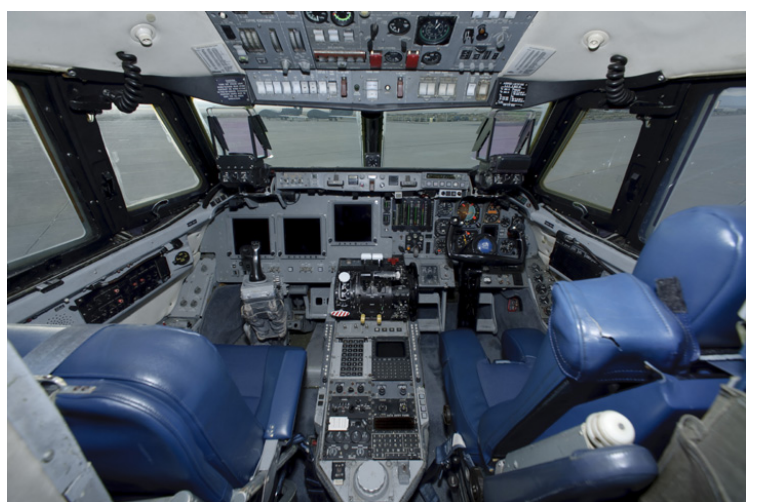

(b) Cockpit of STA with space shuttle configuration

Figure 2.5: Grumman C-11A STA variable stability aircraft [2]

The final notable development from NASA is the McDonnell Douglas FA-18A Full-scale Advanced System Testbed (FAST) as depicted in Figure 2.6 in 2012. It is an highly instrumental aircraft with a digital fly-by-wire flight control system. Since it is also an explicit model-following system, it has many different uses. It was recently used to investigate whether an aircraft can also validate the stability of rocket control logic. One also investigated new flight control laws to use the control surfaces in such a way that fuel consumption during cruise can be reduced by reducing aerodynamic drag. This might be of great interest for future implementation in the civil market.

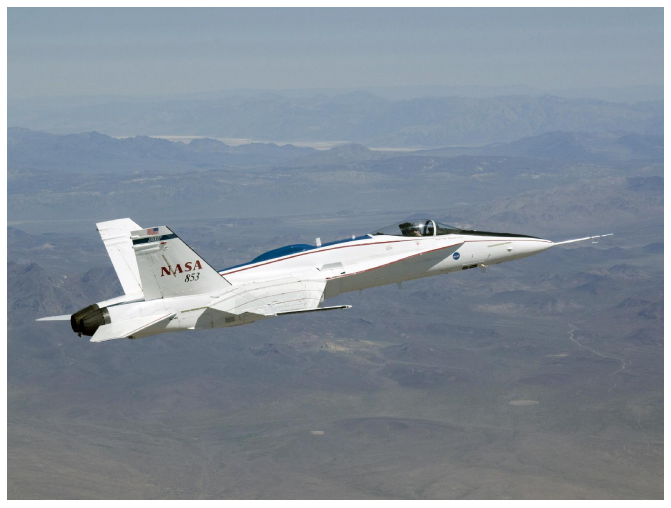

Figure 2.6: McDonnell Douglas FA-18A FAST [2] 


\subsection{Variable stability methods}

A brief overview on different variable stability projects is presented in Section 2.1. This section is dedicated to investigating the different methods used to achieve a variable stability aircraft. Firstly, the response feedback method is investigated. Secondly, both implicit and explicit model-following are researched. Finally dynamic inversion is explained, investigating linear, non-linear and incremental non-linear dynamic inversion. By comparing the different methods, a recommendation is made for future development.

\subsubsection{Response feedback}

The response feedback method is the most basic form of achieving a variable stability system. It uses the response of an aircraft and feeds it back into the aircraft in order to modify its control inputs. A graphical representation of the response feedback system is given in Figure 2.7. The goal of this system is to change the effective dynamics between the pilot's input $u_{p}$ and the state vector $x$.

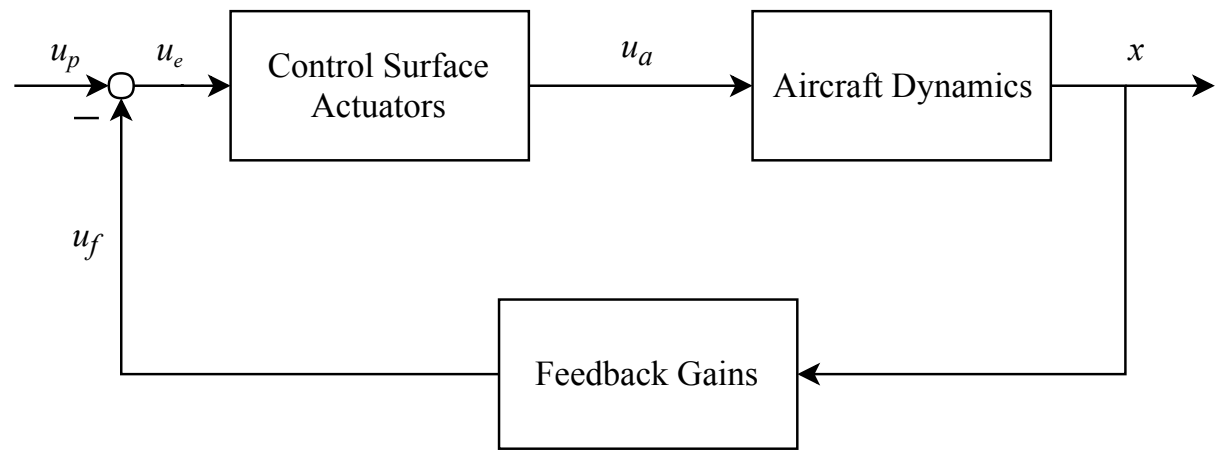

Figure 2.7: Response feedback system block diagram

The mathematics concerning this method are given in Equations 2.1 to 2.3. Equation 2.1 shows the general state space form of aircraft motion, where $A$ is the aircraft's state matrix, $B$ is the input matrix, $u_{e}$ is the input error, $x$ is the state vector and $\dot{x}$ its derivative. Note that this is a model of the aircraft, since the actual aircraft is non-linear. The assumption here is that the response of the linearised system is close to the response of the actual non-linear system. [5]

$$
\dot{x}=A x+B u_{e}
$$

The $A$ matrix holds the coefficients that are representing the aircraft's stability derivatives. Changing these coefficients would mean that we change the actual aircraft. The input error $u_{e}$ consists of two parts as presented in Equation 2.2, where $u_{f}$ is the feedback of the aircraft response, $u_{p}$ is the pilot input and $K^{T}$ is the transpose of the gain matrix $K$.

$$
u_{e}=u_{p}-u_{f}=u_{p}-K^{T} x
$$

If Equation 2.1 and Equation 2.2 are combined, Equation 2.3 is found. One can see that the response of the total system to pilot inputs can be changed by manipulating the gain matrix.

$$
\dot{x}=A x+B\left(u_{p}-K^{T} x\right)=\left(A-B K^{T}\right) x+B u_{p}
$$

The most basic method for controlling this system is to change the eigenvalues through a feedback gain structure. Control theory makes it possible to chose an idealised set of pole locations and to calculate the corresponding feedback gains. One big disadvantage of this method, however, is that the calculated feedback gains only hold for one specific point in the flight envelope. The feedback gains can be calculated using the Bass-Gura method if the aircraft dynamics are in the companion form, but often this is not the case. The Ricatti equation is suggested by literature to find the appropriate gains. This is a set of non-linear differential equations that have to be solved to find the Ricatti gains as explained by Nelson [5]. A full derivation of the Ricatti equation is given by Bryson and Ho [6].

Given an initial state $x\left(t_{0}\right)$, an input vector has to be found in such a way that Equation 2.4 is minimised, where function $g$ is dependent on state vector $x$, time $t$ and the input $u$. 


$$
J=\int_{t_{0}}^{t_{f}} g(x, u, t)
$$

The functional form of the performance index can be expressed in many different forms, but the most useful one is the quadratic index as given in Equation 2.5, where $Q$ is the weighting matrix. This gives a Linear Quadratic (LQ) optimisation approach.

$$
J=\int_{t_{0}}^{t_{f}} x^{T} Q x d t
$$

It is desirable to add a penalty for physical constraints such as the usage of control energy, since this is required by many practical control problems. The Performance Index (PI) can be rewritten into Equation 2.6, where $R$ is another weighting matrix.

$$
J=\int_{t_{0}}^{t_{f}}\left(x^{T} Q x+u^{T} R u\right) d t
$$

Now the non-linear differential equation for the Ricatti gains can be found in Equation 2.7, where $S$ is the Ricatti matrix, a symmetric, positive and definite matrix.

$$
\frac{d S(t)}{d t}=S(t) B R^{-1} B^{T} S(t)-S(t) A-A^{T} S(t)-Q
$$

The time-varying gains are related to the Ricatti matrix as given in Equation 2.8.

$$
k(t)=R^{-1} B^{T} S(t)
$$

For the case where the final time $t_{f}$ approaches infinity, the Ricatti gain matrix becomes a constant matrix which reduces Equation 2.7 to Equation 2.9. This equation is a set of non-linear algebraic equations in terms of the Ricatti gains.

$$
S B R^{-1} B^{T} S-S A-A^{T} S-Q=0
$$

One disadvantage of the response feedback method, is that it is not able to directly follow the input dynamics from another system. As an example, the yaw-motion could not be followed correctly in research from Germann [7]. This is because of the presence of the host dynamics, or in other words, the dynamics of the aircraft that is flown. To cancel these host dynamics, some other method is required. This is investigated in subsection 2.2.3.

However, response feedback has shown to be effective in changing the stability and handling quality. Pilots are able to identify changes in handling qualities caused by the response feedback, even in a fixed-base simulation. For small changes in the configuration (close test points), pilots are not able to correctly identify how the CAP and damping changes. For bigger changes in configuration (far test points), pilots are able to identify the general direction of the change in CAP and damping. However, they are not able to pinpoint the exact changed values. [3] 


\subsubsection{Model-following}

The second method to achieve a variable stability aircraft is called the model-following technique. This technique is a powerful one, since it allows for an aircraft to respond like a predetermined model. This can be beneficial when designing aircraft to meet the specifications determined by regulations. Two main different strategies exist whilst designing a model-following control system, being implicit model-following and explicit model-following. They are initially described by Armstrong [8], Kreindler and Rothschild [9] and O’Brien and Broussard [10]. Both methods will be discussed in this section.

\section{Implicit model-following}

The implicit model-following technique, also known as the model in the performance index technique, is a method where the desired model does not appear in the control structure. However, the desired response is acquired by selecting the weighting matrices in the PI in a meaningful way. Suppose an aircraft can be described by Equations 2.10-2.12.

$$
\begin{gathered}
\dot{x}=A x+B u \\
y=C x \\
z=H x
\end{gathered}
$$

Now it is desired to follow the performance output as given by Equation 2.13. In this case the state matrix $A_{m}$ has its poles positioned in such a way that the desired handling qualities are acquired.

$$
\dot{z}_{m}=A_{m} z_{m}
$$

The performance output will satisfy the differential equation as given in Equation 2.13, thus it is possible to define the control error as in Equation 2.14, where $\dot{z}=H \dot{x}=H A x+H B u$.

$$
e=\dot{z}-A_{m} z
$$

The error has to be minimised without using too much control energy, so the input $u$ is used to minimise the PI. We obtain Equation 2.15. Note that the PI from Equation 2.6 is changed from a finite time-span to time at infinity.

$$
J=\frac{1}{2} \int_{0}^{\infty}\left[\left(H A x+H B u-A_{m} H x\right)^{T} Q\left(H A x+H B u-A_{m} H x\right)+u^{T} R u\right] d t
$$

Equation 2.15 can be rewritten into Equation 2.16, where $Q^{\prime}, W$ and $R^{\prime}$ are redefined as in Equation 2.17.

$$
\begin{gathered}
J=\frac{1}{2} \int_{0}^{\infty}\left(x^{T} Q^{\prime} x+2 x^{T} W u+u^{T} R^{\prime} u\right) d t \\
Q^{\prime}=\left(H A-A_{m} H\right)^{T} Q\left(H A-A_{m} H\right) \\
W=\left(H A-A_{m} H\right)^{T} Q H B \\
R^{\prime}=\left(B^{T} H^{T} Q H B+R\right)
\end{gathered}
$$

The additional term that can be found in $W$ is a cross-weighting between the input $u$ and the state vector $x$. There are multiple solution techniques available to solve Equation 2.16 using the Layapunov and Hamiltonian equations. A complete derivation of this gradient-based solution technique is given in the book by Stevens et al. [11]. The modified design equations are given in Equations 2.18-2.20, where $A_{c}=A-B K C$.

$$
\begin{gathered}
0=A_{c}^{T} P+P A_{c}+Q+C^{T} K^{T} R K C-W K C-C^{T} K^{T} W^{T} \\
0=A_{c} S+S A_{c}^{T}+X \\
0=R K C S C^{T}-(P B+W)^{T} S C^{T}
\end{gathered}
$$


In this solution the optimal cost is given by $J=\frac{1}{2} \operatorname{tr}(P X)$ (using the trace identity of $P X$ ) and the optimal output feedback gains for implicit model-following are described by Equation 2.21. It is necessary to solve these design equations using $Q^{\prime}, W$ and $R^{\prime}$ as defined above.

$$
u=-K y
$$

The implicit model-following technique is nothing but a basic method where gains are chosen to reach the desired behaviour. However, for two reasons this system has an undesirable structure from the perspective of aircraft control. The first reason is that it is required to have derivatives of the performance output $z$ directly available. The second reason is that this technique is only valid for linear or linearised systems, where in an ideal situation model-following would be achieved for a non-linear system.

\section{Explicit model-following}

In explicit model-following, the model to be followed is placed inside the control logic as a feedforward compensator. Explicit model-following is also known as model in the system control. There are two design techniques using explicit model-following, being regulation and tracking. The tracking model-following technique shows more promise for the variable stability system as will be shown later on, but for completeness they will both be described here.

\section{Regulation}

In regulation, the model in the system control is used to minimise the model mismatch error. Let the aircraft be described as Equations 2.10-2.12. The model that has to be followed can be described as in Equations 2.22-2.24. Note that the model has no reference input, since the regulation problem is discussed here. This essentially means that the system should have the same unforced response as the model, which translates to suitable locations of the poles.

$$
\begin{aligned}
& \dot{x}_{m}=A_{m} x_{m} \\
& z_{m}=H_{m} x_{m} \\
& y_{m}=C_{m} x_{m}
\end{aligned}
$$

It is desired to select the system control $u$ in such a way that the performance output $z$ matches the model output $z_{m}$. This comes down to minimising the model mismatch error as in Equation 2.25.

$$
e=z_{m}-z=H_{m} x_{m}-H x
$$

The PI as in Equation 2.26 is selected to achieve the control objective, with $Q>0$ to ensure that all components of the error vanish and $R>0$.

$$
J=\frac{1}{2} \int_{0}^{\infty}\left(e^{T} Q e+u^{T} R u\right) d t
$$

This model-matching problem can be converted to fit the solution technique described before in Equations 2.18-2.20. First the augmented state $x^{\prime}=\left[x^{T} x_{m}^{T}\right]^{T}$ is defined. Now the augmented system can be defined in Equations 2.27-2.28.

$$
\begin{gathered}
\dot{x}^{\prime}=\left[\begin{array}{cc}
A & 0 \\
0 & A_{m}
\end{array}\right] x^{\prime}+\left[\begin{array}{c}
B \\
0
\end{array}\right] u \equiv A^{\prime} x^{\prime}+B^{\prime} u \\
y^{\prime}=\left[\begin{array}{c}
y \\
y_{m}
\end{array}\right]=\left[\begin{array}{cc}
C & 0 \\
0 & C_{m}
\end{array}\right] x^{\prime} \equiv C^{\prime} x^{\prime}
\end{gathered}
$$

Using these definitions, the error can be defined as in Equation 2.29.

$$
e=\left[\begin{array}{ll}
-H & H_{m}
\end{array}\right] x^{\prime} \equiv H^{\prime} x^{\prime}
$$

Now the PI from Equation 2.26 can be rewritten to Equation 2.30 using 2.31 . 


$$
\begin{gathered}
J=\frac{1}{2} \int_{0}^{\infty}\left(\left(x^{\prime}\right)^{T} Q^{\prime} x^{\prime}+u^{T} R u\right) d t \\
Q^{\prime}=\left[\begin{array}{cc}
H^{T} Q H & -H^{T} Q H_{m} \\
-H_{m}^{T} Q H & H_{m}^{T} Q H_{m}
\end{array}\right]
\end{gathered}
$$

The form of the output control law uses not only the system output but also the model output, as can be seen in Equation 2.32. This essentially means that the model acts as a compensator to drive the system states to zero in such a way that the performance output $z$ follows the model output $z_{m}$. [11]

$$
u=-K^{\prime} y^{\prime} \equiv-\left[\begin{array}{ll}
K_{p} & K_{m}
\end{array}\right] y^{\prime}=-K_{p} y-K_{m} y_{m}
$$

\section{Tracking}

In tracking, the model in the system control is used to follow a model which is driven by a reference input. This added reference input complicates the solution compared to the regulation problem, but makes it much more useful as a control system in aircraft. Again, the system can be determined as Equation 2.22-2.24. The model that has to be followed is defined as Equation 2.33-2.34.

$$
\begin{gathered}
\dot{x_{m}}=A_{m} x_{m}+B_{m} r \\
z_{m}=H_{m} x_{m}
\end{gathered}
$$

Using the same approach as for the regulation problem, Equation 2.35 is found.

$$
\dot{x}^{\prime}=\left[\begin{array}{cc}
A & 0 \\
0 & A_{m}
\end{array}\right] x^{\prime}+\left[\begin{array}{l}
B \\
0
\end{array}\right] u+\left[\begin{array}{l}
0 \\
B
\end{array}\right] r \equiv A^{\prime} x^{\prime}+B^{\prime} u+G^{\prime} r
$$

If the derivation from the regulation problem would be used, the PI would contain a term in $r$, for which it is not easy to solve according to Stevens et al. [11]. However, Franklin et al. [12] designed a convenient technique to solve this problem, being the command generator tracker technique. In their approach, the reference input is replaced by a generator system. This approach is elaborated on below to show the its advantages.

Suppose that the reference command $r$ satisfies the differential equation as in Equation 2.36 for a given degree $d$ and set of coefficients $a_{i}$. Most command signals of interest will satisfy such an equation (think of a unit step or ramp function). Equation 2.36 is also known as the command generator system.

$$
r^{(d)}+a_{1} r^{(d-1)}+\ldots+a_{d} r=0
$$

Now the command generator characteristic polynomial can be defined as in Equation 2.37.

$$
\Delta(s)=s^{d}+a_{1} s^{d-1}+\ldots+a_{d}
$$

For $r$ in the time domain, Equation 2.38 can be used.

$$
\Delta\left(\frac{d}{d t}\right) r=0
$$

Multiplying Equation 2.38 with 2.35 results in a modified system as given in Equation 2.39, where the modified state $\xi$ and control input $\mu$ are as given in Equations 2.40-2.41.

The main reason for these manipulations is that Equation 2.39 does not contain the reference command anymore. In contrast to the original system, this newly acquired system can be used in Equation 2.18-2.20 to find the solution for the optimal feedback gains. A complete derivation of this process is given by Stevens et al. [11], but not considered of direct interest for this research.

$$
\begin{gathered}
\dot{\xi}=A^{\prime} \xi+B^{\prime} \mu \\
\xi=\Delta\left(\frac{d}{d t}\right) x^{\prime}=\left(x^{\prime}\right)^{(d)}+a_{1}\left(x^{\prime}\right)^{(d-1)}+\ldots+a_{d} x^{\prime}
\end{gathered}
$$




$$
\mu=\Delta\left(\frac{d}{d t}\right) u=u^{(d)}+a_{1} u^{(d-1)}+\ldots+a_{d} u
$$

The model-following command generator tracker block diagram is of interest to this research. The main advantage of this system is that it automatically adds the compensator of an appropriate structure to guarantee that the system has the correct type for tracking. The resulting model for a $d$ of three can be seen in Figure 2.8.

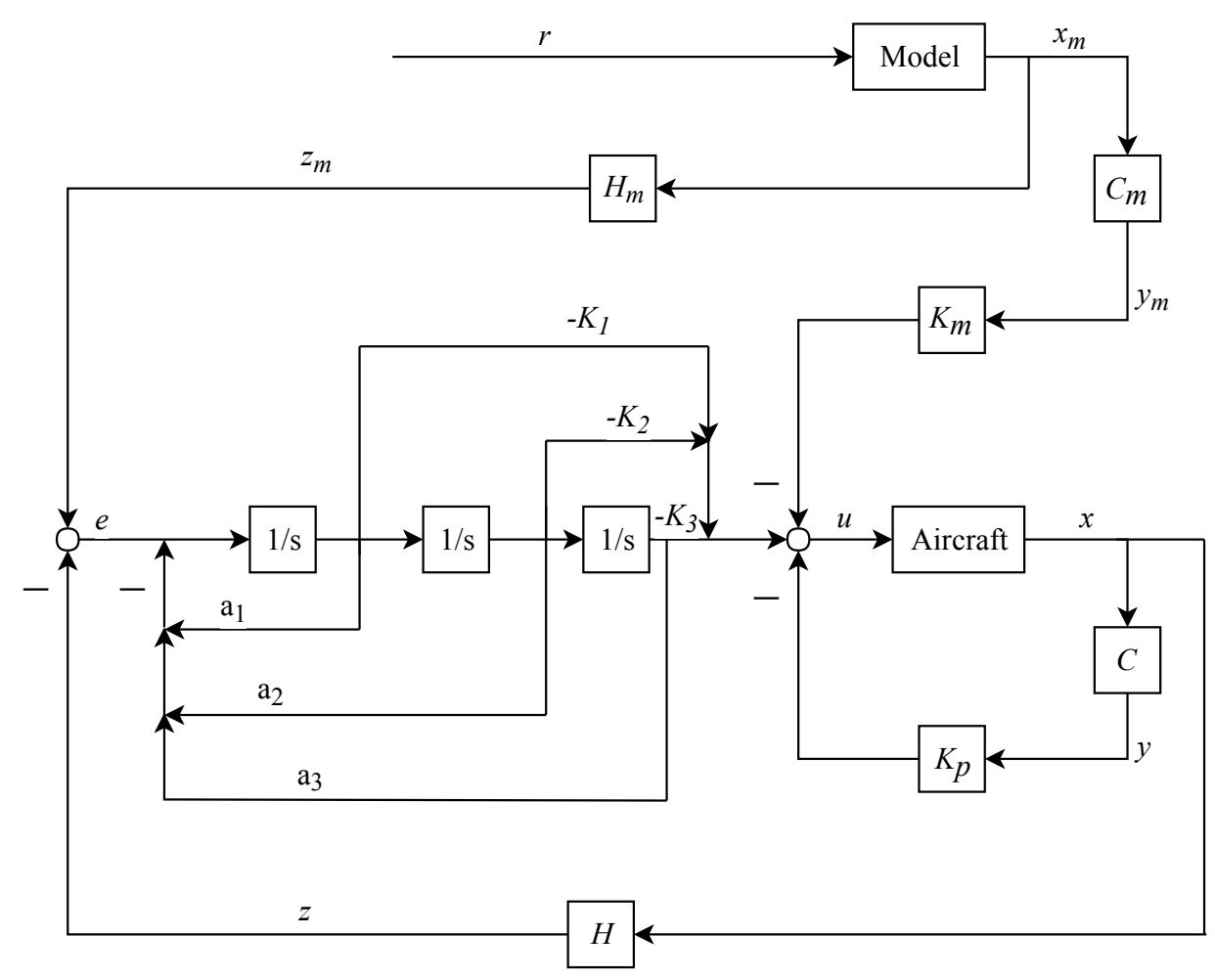

Figure 2.8: Explicit model-following command generator tracker for $d=3$

For aircraft applications $r$ is usually the pilot's command input. This means that for control system design, it is not necessary to determine the actual coefficients $a_{i}$, as done by Kreindler and Rothschild [9]. Instead, the performance objectives should be used as a design metric to select $\Delta(s)$. If the aircraft should follow a position command, the command generator $\dot{r}=0$ should be selected. However, if the aircraft should follow a rate (velocity) command, $\ddot{r}=0$ can be used. When the actual command is applied (which does not have to be a unit step or a unit ramp), the aircraft will exhibit the correct closed-loop behaviour. 


\subsubsection{Dynamic inversion}

The previously presented control designs all have the same drawback. Since an aircraft is generally a nonlinear system, applying the design tools presented before requires designing several linear controllers and gain scheduling these over the operating regime of the aircraft. A technique is required which deals with the known non-linearities of the aircraft dynamics by using them in the controller to improve the performance of the total system. This technique is called Dynamic Inversion and has grown in popularity over the past years. It is a form of model-following, but it reaches this goal in a different way compared to the previously shown methods. To show the general concept, firstly the linear dynamic inversion principle is explained as described by Stevens et al. [11]. Secondly, the non-linear dynamic inversion concept is presented. Finally, a new iteration of the non-linear dynamic inversion is elaborated upon, being the Incremental Non-linear Dynamic Inversion (INDI) method.

\section{Linear dynamic inversion}

The aircraft can be described by the state-variable form as in Equation 2.42-2.43, with state $x$, control input $u$ and output $y$.

$$
\begin{gathered}
\dot{x}=A x+B u \\
y=C x
\end{gathered}
$$

It is assumed that the system is square, which means that the vectors $u$ and $y$ should have the same dimension. For aircraft, this is often the case, since normal aircraft configurations have one control actuator per degree of freedom. Next to this, the state $x$ is assumed to be available for feedback purposes. For a square system, the output $y$ should follow the desired reference command $r$. The tracking error can be defined as in Equation 2.44.

$$
e(t)=r(t)-y(t)
$$

The concept of dynamic inversion uses differentiation of the output $y$ until the control $u$ has appeared in the expression for the derivative. This is firstly introduced by Slotine and Li [13] and is called input-output feedback linearisation. Equations 2.45-2.46 show the first and second derivative of the output $y$.

$$
\begin{gathered}
\dot{y}=C \dot{x}=C A x+C B u \\
\ddot{y}=C \ddot{x}=C A \dot{x}+C B \dot{u}=C A^{2} x+C A B u
\end{gathered}
$$

In the first derivative, the input $u$ appears if the matrix $C B$ is not equal to zero. Since the system is square, one can also state that if $C B$ is nonsingular, the second derivative is not needed. However, if $C B=0$, the second derivative is used and it has to be checked if $C A B$ is not equal to zero. As already described before, for regular aircraft configurations, an aircraft has one actuator for each degree of freedom. This implies that generally, $C B$ is nonsingular. Higher order derivatives after Equation 2.45 are not required.

Now it is required to define an auxiliary input $v$ in such a way that the terms $C A x$ and $C B$ do not appear in the error equation. Equation 2.47 is the starting point and can be rewritten to the control input $u$ as given in Equation 2.48 .

$$
\begin{gathered}
v=\dot{y}-\dot{r}=C B u+C A x-\dot{r} \\
u=(C B)^{-1}(\dot{r}-C A x+v)
\end{gathered}
$$

Substituting Equation 2.48 in Equation 2.45 gives Equation 2.49.

$$
\dot{y}=C A x+C B\left[(C B)^{-1}(\dot{r}-C A x+v)\right]=C A x+\dot{r}-C A x+v=\dot{r}+v
$$

Finally, combining Equation 2.49 and Equation 2.42 gives the error dynamics for the system as given in Equation 2.50. To finalise the dynamic inversion design, the auxiliary input $v$ has to be selected in such a way that the system is stable. To achieve a stable system that cancels the host dynamics, the selection of $v$ does 
not have to be complicated and a simple gain $K$ will suffice as given in Equation 2.51, which results in the closed-loop error dynamics as given in Equation 2.52. The selection of $K$ should be performed according to the requirements from Standard [14] to ensure the flying quality requirements are meeting the specifications. The overall dynamic inversion control input can be described by Equation 2.53.

$$
\begin{gathered}
\dot{e}=-v \\
v=K e \\
\dot{e}=-K e \\
u=(C B)^{-1}(\dot{r}+K e-C A x)
\end{gathered}
$$

The control scheme for the linear dynamic inversion is depicted in Figure 2.9. Equation 2.51 is represented in the control scheme by the outer tracking loop, which uses proportional feedback. The inner control loop, or the feedback linearisation loop represents Equation 2.48 and requires full state-variable feedback. It has the function to make the system from the auxiliary input $v$ to the output $y$ appear like a linear system with the poles at the origin.

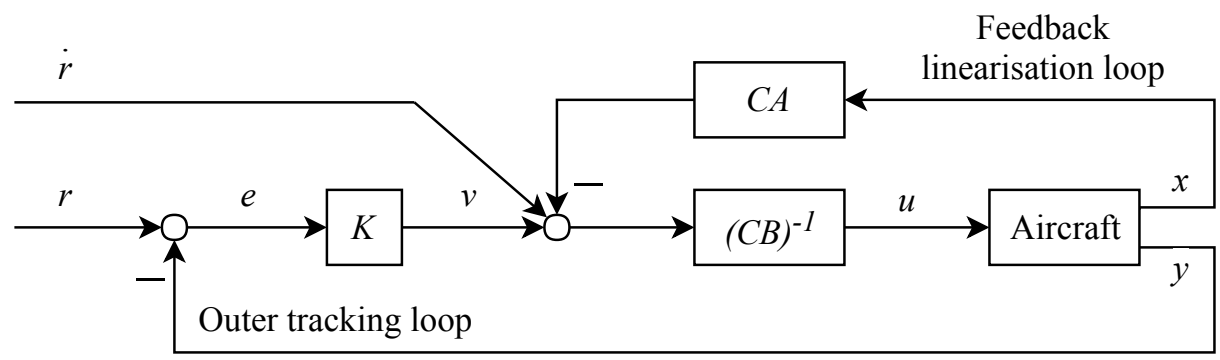

Figure 2.9: Linear dynamic inversion controller block diagram

It should be noted that for implementation of this linear dynamic inversion control, one must know $C A$ and $C B$. This essentially means that a model of the aircraft dynamics is actually built into the controller. Next to this, full state feedback is required for the feedback linearisation loop, which is not always available when flying an aircraft.

This linear dynamic inversion has been implemented by Ko et al. [15]. They used a model of the Korean next generation fighter and tried to make it follow the dynamics of the F-16 fighter aircraft. Ko and Park [16] introduced an updated version of their system, which now also included a switching mechanism between the normal flight computer and the dynamic inversion model. Based on fairly simple switching, they managed to get a stable response when turning on or off the variable stability system. The fader equation is given in Equation 2.54 where $\delta_{I F S}$ and $\delta_{V S S}$ are the actuator commands calculated by the baseline flight control law and the dynamic inversion control law respectively. The fading factor $\alpha_{f}$ is a value between 0 and 1 which changes over time.

$$
\delta_{\text {fader }}(t)=\left(1-\alpha_{f}(t)\right) \delta_{I F S}(t)+\alpha_{f}(t) \delta_{V S S}(t)
$$

Finally Ko and Park [17] introduced the normal load to their initial variable stability control system, that only could follow pitch, roll and yaw. Their system works very well in the simulations shown in the paper, but they fail to mention the downsides of linear dynamic inversion. Their tests are currently only run in the simulator and they do not take into account the actual implications of moving from the simulator to a real aircraft. If they want to fly with the system, they have to ensure that the model is properly linearised for the flight conditions in which they are operating. Also the model from the next generation Korean fighter aircraft has 
to be of a high enough accuracy to ensure that the original dynamics of the aircraft are cancelled properly. If this linear model is not accurate enough, it might introduce instability when the poles are not properly placed at zero.

\section{Non-linear dynamic inversion}

The advantage of dynamic inversion is that the technique can directly be applied to non-linear systems. Since an aircraft is inherently a non-linear system, this should be investigated in more detail. The aircraft can be described by the non-linear state variable form as given in Equations 2.55-2.56, with state $x$, control input $u$ and output $y$. As for the linear dynamic inversion, it is assumed that the system is square and that the entire state is available for feedback purposes.

$$
\begin{gathered}
\dot{x}=f(x)+g(x) u \\
y=h(x)
\end{gathered}
$$

The derivation for the non-linear dynamic inversion is similar to the linear counterpart. First the tracking error is defined as in Equation 2.57. The output $y$ is differentiated to obtain the result as in Equation 2.58.

$$
\begin{gathered}
e(t)=r(t)-y(t) \\
\dot{y}=\frac{\partial h}{\partial x} \dot{x}=\frac{\partial h}{\partial x} f(x)+\frac{\partial h}{\partial x} g(x) u \equiv F(x)+G(x) u
\end{gathered}
$$

Now the control input $u$ can be defined by Equation 2.59 with the auxiliary input $v$. Note that the error dynamics for both the linear as the non-linear dynamic inversion are equivalent, which means the auxiliary input can again be defined by using a simple gain $K$ as given in Equation 2.60.

$$
\begin{gathered}
u=G^{-1}(x)[-F(x)+\dot{r}+v] \\
v=K e
\end{gathered}
$$

Now the overall non-linear dynamic inversion controller is given by Equation 2.61.

$$
u=G^{-1}(x)[-F(x)+\dot{r}+K e]
$$

The graphical representation of the control scheme can be found in Figure 2.10. Again the feedback linearisation loop has the function to make the system from the auxiliary input $v$ to the output $y$ appear like a system with the poles at the origin. It should be noted that the control logic uses a model of the aircraft dynamics, since it requires $F(x)$ and $G(x)$. Therefore it is required to know the equations for the non-linear dynamics of the aircraft. In practice this means that often full lookup tables have to be implemented in the controller, but with the current computational power this is possible. Miller [18] shows such an implementation for the FAST system from NASA, with hardware in the loop simulation experiments. However, recent developments within dynamic inversion show that the full model of the aircraft is not required in the loop anymore. This will be explained in the next section.

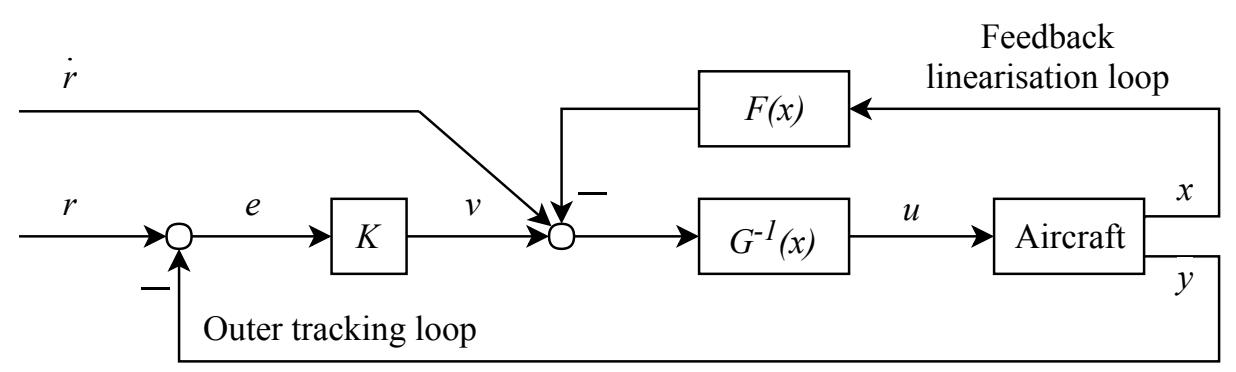

Figure 2.10: Non-linear dynamic inversion controller block diagram 


\section{Incremental non-linear dynamic inversion}

Incremental non-linear dynamic inversion (INDI or sometimes called sensor-based dynamic inversion) is the most recent development in the field of dynamic inversion. It uses the advantages from the dynamic inversion, where the host dynamics are cancelled, but does not require the full non-linear aircraft model anymore.

The development of these models can be traced back to Smith [19]. He acknowledges the issues with the nonlinear dynamic inversion and proposed a new method that would use acceleration sensors as a replacement for the feedback linearisation loop. The accelerations have to be converted to the equivalent pitch, roll or yaw rate using a moment effectiveness parameter (consisting of the moment due to control surface deflection and the aircraft inertias). He shows that the newly developed control method could provide considerable robustness regarding authority limits, sensor noise and significant variations within the control law (mainly inertias and control powers). The general control scheme can be found in Figure 2.11. Note that this scheme only shows the inner loop, whilst the more recent INDI developments also contain an outer tracking loop.

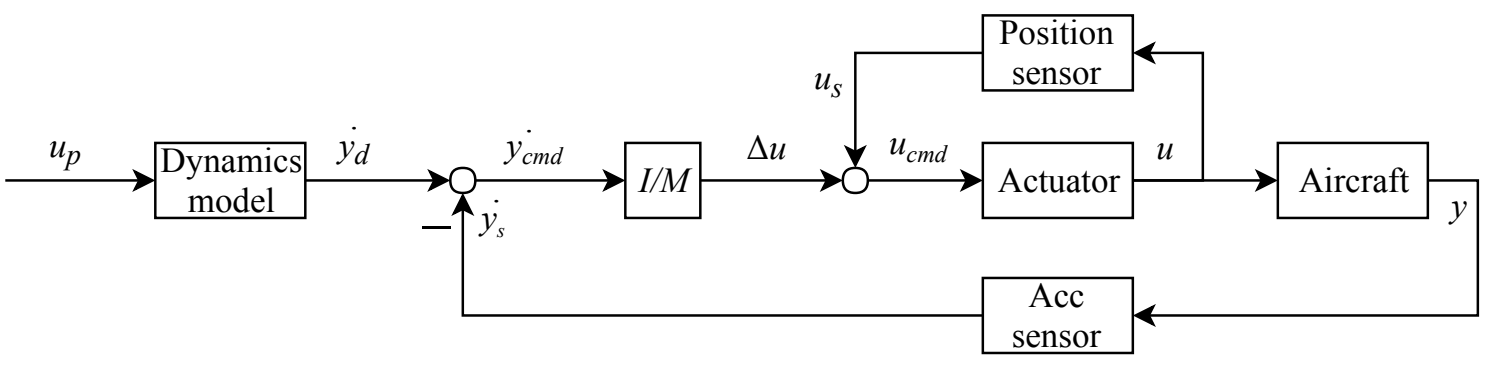

Figure 2.11: Simplified non-linear dynamic inversion controller block diagram

The pilot gives an input up to an a priori known dynamic model and this results in a desired rate output $\dot{y}$, which should comply with the requirements from Standard [14]. The combination of the actuator position sensor and the acceleration sensors make sure that angular accelerations can be matched robustly. The only flight condition related gain that is required, is the moment effectiveness $I / M$. Germann [7] developed a variable stability system for the T-6A Texan II for the American Airforce, based on the above principle. He showed in offline simulations that the Texan II could match the dynamics of the F-16 aircraft. He also noted that more research was required before the theoretical system could be implemented in a flyable solution. He did not mention that he would require some kind of gain scheduling to ensure the moment effectiveness gain would be valid in different flight conditions, since he only tested his simulations at one specific flight condition.

Bacon and Ostroff [20] used the sensor based non-linear dynamic inversion method to design a re-configurable flight control system in case of system failure. They acquired the angular accelerations by combining linear accelerations and differentiating the angular rates. This created noise and was considered to be sufficient but not optimal, since the differentiation also introduced some time delays. Cox and Cotting [21] continued the development of the ideas from Smith, Bacon and Ostroff. They extended the simplification to the ground base simulators at NASA Dryden. The main takeaway from their work is that the method is viable for realtime aircraft control and simulation, not just in an offline simulation. They did, however, note the problem of noise introduction due to the differentiation of the angular rates.

Simultaneously to the above developments, work on the non-linear dynamic inversion started at Delft University of Technology. Sieberling et al. [22] combined information from previous non-linear dynamic inversion research and created the concept of INDI in combination with angular acceleration prediction (combined known as Predictive Incremental Non-linear Dynamic Inversion (PINDI)). The main advantage of PINDI, which uses a predictive Kalman filter to attain the required angular accelerations, is that it is directly applicable to most systems and does not require angular accelerometers. Sieberling shows robustness of the developed PINDI system compared to the regular non-linear dynamic inversion systems. Note that the only 
reason Sieberling introduced the predictive filter is because the angular accelerations are not readily available. However, in the end of 2017 angular accelerometers are installed in the Cessna Citation II, which makes the predictive Kalman filter obsolete.

Simplício et al. [23] adapted the INDI method to be applicable to helicopters and shows that the helicopter flight control systems can be simplified by making use of the INDI system. Smeur et al. [24] [25] developed the system to be applicable to Micro Aerial Vehicle (MAV)'s. They showed that the INDI controller performs better regarding to gust resistance, compared to a normal Proportional Integral Derivative (PID) controller. Even though they show the robustness of the controller, they also acknowledge the risk of actuator saturation regarding the stability of the system. Since this research focuses on using the fly-by-wire system of the Cessna Citation II to achieve a variable stability in-flight simulator, the limits of the actuators have to be researched when using the fly-by-wire system.

Concluding, INDI shows the highest potential to achieve a variable stability in-flight simulator. It is beneficial over other model-following techniques since it requires almost no gain scheduling (except for a proportional gain for the moment effectiveness) and no full non-linear model of the aircraft is required. This makes the INDI system simpler and easier to apply. INDI shows preference over PINDI, since angular accelerometers are recently installed in the Cessna Citation II. However, actuator limits from the fly-by-wire system and the impact of reaching actuator saturation on stability will both have to be investigated when the new system is developed. 


\subsection{Handling Qualities}

A method is required to determine the varying handling qualities of the Cessna Citation II. In previous research by Mirza et al. [3], a trade-off has been made between the different methods. For the completeness of this literature study, the methods are shortly introduced. Firstly, the CAP is introduced as a measure to objectively determine the handling qualities. Secondly, a method is introduced to transform the High Order System (HOS) of the non-linear aircraft and actuator model to a Low Order Equivalent System (LOES). This is required to calculate the CAP. Thirdly, the Cooper-Harper rating scale is introduced as a more subjective measure by pilots to determine handling qualities. Finally the different pilot manoeuvres are discussed which have to be performed to asses the handling qualities.

\subsubsection{Control anticipation parameter}

The CAP is a longitudinal handling quality criterion which focuses on the short period pitch response of an aircraft. It was originally introduced by Bihrle [26], after which it became the standard measure of longitudinal handling qualities in the MIL-STD-1797A [14]. It is defined for un-augmented aircraft as the ratio of initial pitch acceleration (second derivative of $\theta$ ) to the steady state normal acceleration $n_{z}$ and it is expressed by Equation 2.62. The steady state normal acceleration can be rewritten as in Equation 2.63, where $V$ is the velocity, $g$ is the gravitational acceleration and $q$ is the steady state pitch rate.

$$
\begin{aligned}
& C A P=\frac{\ddot{\theta}(0)}{n_{z_{s s}}} \\
& n_{z_{s s}}=\frac{V}{g} q_{s s}
\end{aligned}
$$

The boundaries of the CAP depend on the aircraft class, the different flight phase operations and the acceptability levels. Sandraey [27] gives a brief overview of these dependencies and they are displayed in Table 2.12.3. The Cessna Citation II is a Class II aircraft and most flight operations will be of the B category. With these boundaries, the specific levels of acceptability are given in Figure 2.12. The CAP is often displayed in conjunction with the short period damping ratio.

Bischoff [28] shows that the CAP can be calculated using the aircraft short period characteristics. It should be noted that this method only holds for aircraft with a classical longitudinal second order response as given in Equation 2.64. Using this knowledge, Bischoff rewrites Equation 2.62 to Equation 2.65.

$$
\begin{gathered}
\frac{q}{\delta_{e}}=\frac{K_{\theta}\left(s+\frac{1}{T_{\theta_{2}}}\right)}{s^{2}+2 \zeta_{s p} \omega_{s p} s+\omega_{s p}^{2}} \\
C A P \approx \frac{\omega_{n_{s p}}^{2}}{n / \alpha} \approx \frac{\omega_{n_{s p}}^{2}}{\frac{V}{g} \frac{1}{T_{\theta_{2}}}}
\end{gathered}
$$

Table 2.1: Aircraft classes [27]

\begin{tabular}{ll}
\hline \hline Class & Aircraft Characteristics \\
\hline I & $\begin{array}{l}\text { Small, light aircraft (maximum take-off mass less than } 6000 \mathrm{~kg} \text { ) with low manoeu- } \\
\text { vrability }\end{array}$ \\
II & $\begin{array}{l}\text { Aircraft of medium weight and low-to-medium manoeuvrability (maximum take- } \\
\text { off mass between } 6000 \text { and } 300000 \mathrm{~kg})\end{array}$ \\
III & $\begin{array}{l}\text { Large, heavy and low-to-medium manoeuvrability aircraft (maximum take-off } \\
\text { mass more than } 30000 \mathrm{~kg})\end{array}$ \\
IV & Highly manoeuvrability aircraft, no weight limit (e.g., acrobatic, missile and fighter) \\
\hline
\end{tabular}


Table 2.2: Flight phase categories [27]

\begin{tabular}{|c|c|}
\hline Category & Example of flight operation \\
\hline A & $\begin{array}{l}\text { (1) air-to-air combat; (2) ground attack; (3) weapon delivery/launch; (4) aerial re- } \\
\text { covery; (5) reconnaissance; (6) in-flight-refuelling; (7) terrain following; (8) anti- } \\
\text { submarine search; (9) close formation flying; (10) low-altitude parachute extraction } \\
\text { system delivery }\end{array}$ \\
\hline B & $\begin{array}{l}\text { (1) climb; (2) cruise; (3) loiter; (4) in-flight refuelling in which aircraft acts as a } \\
\text { tanker; (5) decent; (6) emergency-decent; (7) emergency deceleration; (8) aerial de- } \\
\text { livery }\end{array}$ \\
\hline $\mathrm{C}$ & $\begin{array}{l}\text { (1) take-off; (2) catapult take-off; (3) powered approach; (4) wave-off/go-around; } \\
\text { (5) landing }\end{array}$ \\
\hline
\end{tabular}

Table 2.3: Levels of acceptability [27]

\begin{tabular}{ll}
\hline \hline Level & Definition \\
\hline 1 & Flying qualities clearly adequate for the mission flight phase \\
2 & $\begin{array}{l}\text { Flying qualities adequate to accomplish the mission flight phase, but some increase } \\
\text { in pilot workload on degradation in mission effectiveness, or both, exists }\end{array}$ \\
3 & $\begin{array}{l}\text { Flying qualities such that the aircraft can be controlled safely, but pilot workload is } \\
\text { excessive or mission effectiveness is inadequate, or both. Category A flight phases } \\
\text { can be terminated safely, and Category B and C flight phases can be completed }\end{array}$ \\
\hline \hline
\end{tabular}

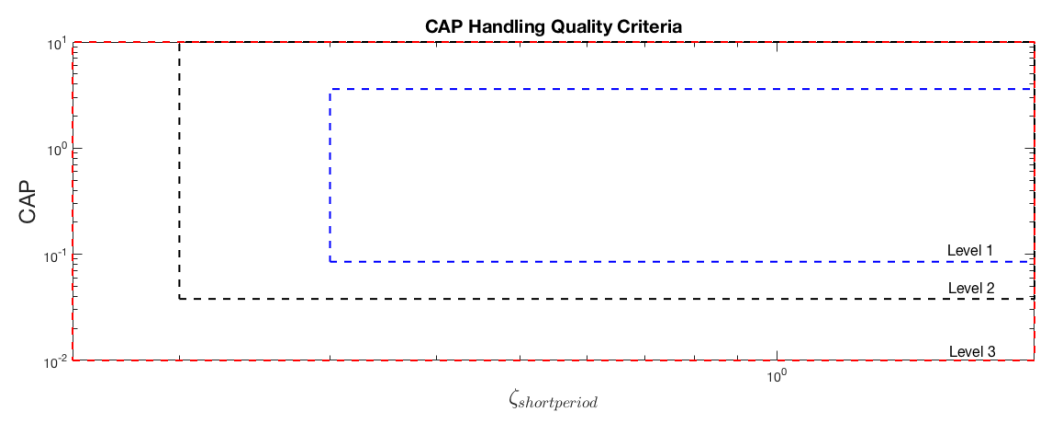

Figure 2.12: CAP short period requirements for Class II, Category B aircraft

Since it is required to model the CAP of the non-linear aircraft and non-linear actuator dynamics, a method is required to model the higher order response to the second order model given in Equation 2.64. This method is explored in the next section.

\subsubsection{High order to low order equivalent system}

Most handling quality criteria were initially developed for un-augmented aircraft for which actuator dynamics were assumed to be negligible. However, both the aircraft as the actuator model are non-linear, higher order models. This implies that the system cannot be linearised to match the form as presented in Equation 2.64. To still be able to use the CAP criterion, another method has to be introduced to find the required short period response parameters.

Di Franco [29] was one of the first to investigate the effects of higher order control system dynamics on the longitudinal handling qualities. He determined that the aircraft's short period response could be represented reasonably well by a time delay and an equivalent second-order term. Di Franco expressed this as in Equation 2.66 . 


$$
\frac{q}{\delta_{e}}=\frac{K_{\theta}\left(s+\frac{1}{T_{\theta_{2}}}\right) e^{-\tau s}}{s^{2}+2 \zeta_{s p} \omega_{s p} s+\omega_{s p}^{2}}
$$

This concept was further developed into the LOES by Hodgkinson et al. [30]. The equivalent system is created by matching the complex freqency response of a HOS with a classical low order system and a time delay term to derive the equivalent short period frequency and damping ratio. These can then in turn be used in Equation 2.65 to calculate the CAP. There are some advantages and disadvantages to the LOES method and they are discussed in more detail by Mirza et al. [3], but they are not considered the scope of this research. To maintain consistency over the research topic at the Delft University of Technology, it is decided to use the same method as followed by Mirza.

There are several techniques available to determine the LOES and their advantages and disadvantages are also discussed by Mirza et al. [3]. The most robust method is the Output Error and Equation Error approach developed by Morelli [31]. His method is briefly explained below.

The LOES method introduced by Morelli uses the equation error and output error parameter estimation in the frequency domain. They are two separate parameter estimation methods. The advantage of the equation error method is that it can estimate the parameters with only the time delay fixed to a specific initial condition, but it is less accurate compared to the output error method. If only the output error method would be used, it would be possible that the solution converges to a local minimum if the wrong initial conditions are chosen. That is why Morelli suggested using the resulting parameters from the equation error estimation as an initial guess for the output error formulation. This way the results with the lowest error can be achieved.

Equation 2.66 is reparametrized to lower the correlation between the parameters $K_{\theta}, \omega_{e}$ and $T_{\theta_{2}}$. Morelli especially noticed the correlation at lower frequencies, which are of high importance for the correct estimation. He introduced Equation 2.67 with Equation 2.68 as the parameter definitions. The reparametrized equation can in its turn be converted into the Fourier transform as shown in Equation 2.69. This formulation is required to perform the correct optimisation.

$$
\begin{gathered}
\frac{q}{\delta_{e}}=\frac{\left(b_{1} s+b_{0}\right) e^{-\tau s}}{s^{2}+a_{1} s+a_{0}} \\
b_{1}=K_{\theta} \quad b_{0}=\frac{K_{\theta}}{T_{\theta_{2}}} \\
a_{1}=2 \zeta_{e} \omega_{e} \quad a_{0}=\omega_{e}^{2} \\
\frac{q}{\delta_{e}}=\frac{\left(b_{1} j \omega+b_{0}\right) e^{-\tau j \omega}}{(j \omega)^{2}+a_{1} j \omega+a_{0}}
\end{gathered}
$$

The cost function for the equation error estimation method is given by Equation 2.70. It considers the error in the frequency domain between the measured pitch rate $\tilde{q_{E_{i}}}$ and the estimated pitch rate $\hat{\tilde{q}}_{i}$ over the $m$ frequencies used in the frequency transform. $J$ is the final sum of the frequency weighted errors in the equation error formulation. The estimated pitch rate is calculated by evaluating Equation 2.69 with the estimated parameters and the measured deflection of the elevator $\delta_{e}$.

$$
J_{E E}=\frac{1}{2} \sum_{i=1}^{m}\left|\omega_{i}^{2} \tilde{q_{E}}-\omega_{i}^{2} \hat{\tilde{q}}_{i}\right|^{2}
$$

The parameter estimation itself can be performed by any non-linear estimation routine like the simplex method, the modified Newton-Raphson or the Levenberg-Marquardt. Morelli suggests using the NewtonRaphson method with an initial fixed time delay $\tau$, since this reduced the problem to a linear parameter estimation problem involving complex numbers. When initial parameters with a fixed time delay are found, the time delay can be optimised to lower the cost function even further. This time delay can again be fixed to find new parameter values for the optimised time delay. This iterative loop is continued until the cost is not lowered anymore by a significant margin. 
The output error method uses the initial parameters from the equation error method in its parameter estimation. The cost function for the output error is given in Equation 2.71. The only difference is that frequency weighting is not applied anymore, which also explains why the parametrization from the output error method can be more accurate. Again, the estimated pitch rate is calculated by evaluating Equation 2.69 with the estimated parameters and the measured deflection of the elevator $\delta_{e}$. It can be solved with the same nonlinear optimising methods as presented for the equation error method, but $\tau$ does not have to be fixed anymore to find the global minimum. [31]

$$
J_{O E}=\frac{1}{2} \sum_{i=1}^{m}\left|\tilde{q_{E_{i}}}-\hat{\tilde{q}}_{i}\right|^{2}
$$

Having defined a method for objective handling quality determination, the subjective counterpart is discussed next.

\subsubsection{Cooper-Harper rating scale}

The Cooper-Harper rating scale is the accepted standard for subjective handling quality measurement. Cooper and Harper [4] developed a decision tree rating scale as a tool to assess aircraft handling quality. The scale takes qualitative pilot comments and translates them into a quantitative scale. The decision tree can be found in Figure 2.13. It can be used in experiments to find the handling qualities as experienced by the pilots.

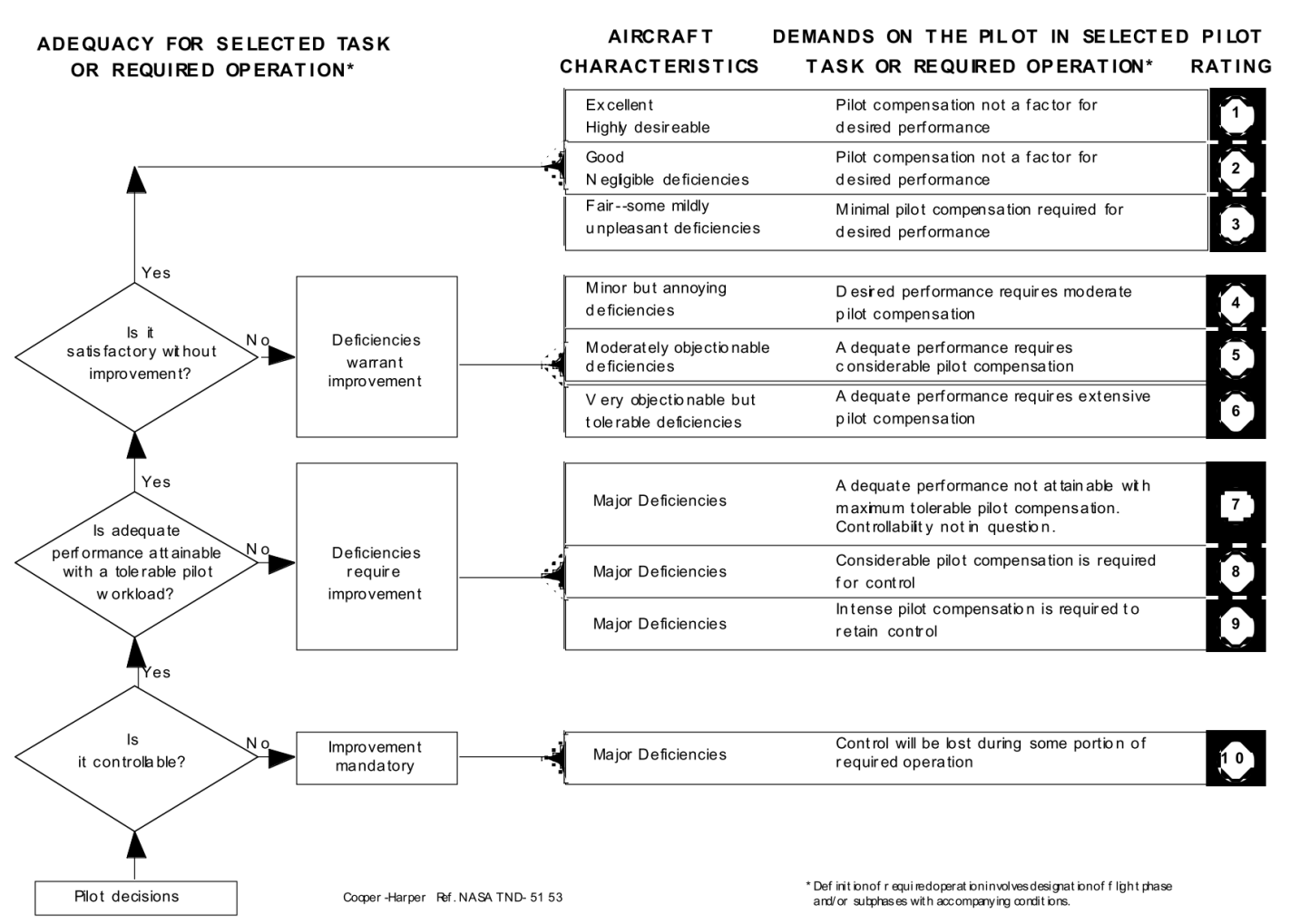

Figure 2.13: Cooper-Harper rating scale decision tree [4]

The rating scale is used by Mirza et al. [3] to check if changes in CAP could be correctly identified by pilots. CAP points that were positioned further from the original aircraft configuration could not be correctly detected, but points close to the original configuration could. One downside of Mirza's experiment was that it was performed without motion, which probably reduces the capacity of the pilots to detect changes. Next to this, pilots are probably not experienced in flying aircraft outside original operating points, so they have less experience in detecting such a configuration which increases the chances of erroneous ratings. Also the control group in Mirza's experiment only consisted of 3 pilots, which is quite small to draw statistically significant conclusions. 


\subsubsection{Pilot manoeuvres}

When designing an experiment to determine the handling qualities of an aircraft, the manoeuvre selection is one of the main experiment design decisions. In the longitudinal handling quality experiment by Mirza it was decided to have the pilots climb 1000 feet with rate of climb between 500 and 1000 feet/min. However, more different manoeuvres are available to determine the handling qualities. This has been researched by Wilson et al. [32] in more detail. A selection guide has been created to select the specific manoeuvre for the aircraft attribute or handling quality that has to be tested. Different manoeuvres and their characteristics are displayed in Figure 2.14.

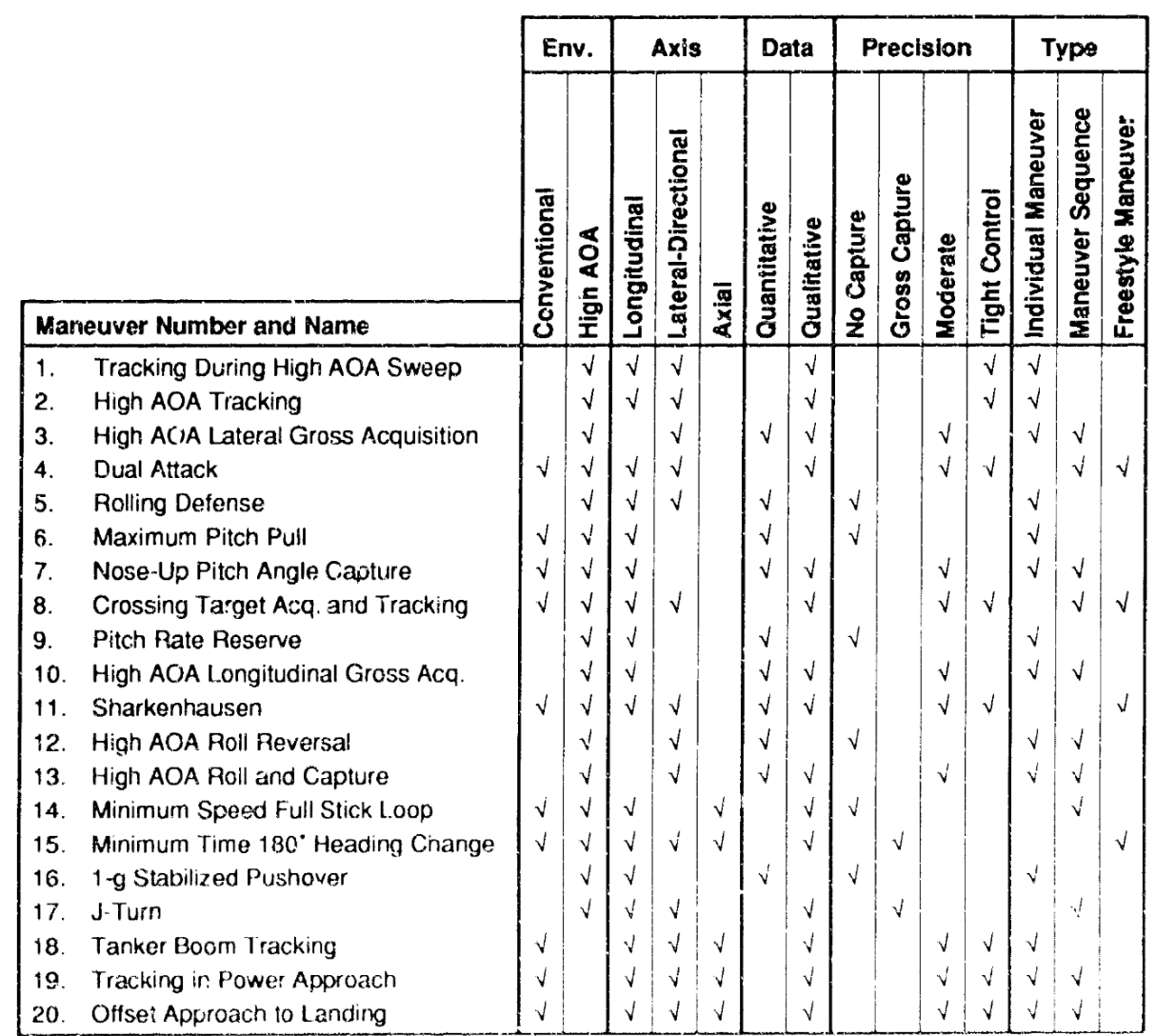

Figure 2.14: General manoeuvre characteristics [32]

These manoeuvres can be used to test different design parameters or handling qualities of the aircraft. This is depicted in Figure 2.15. 


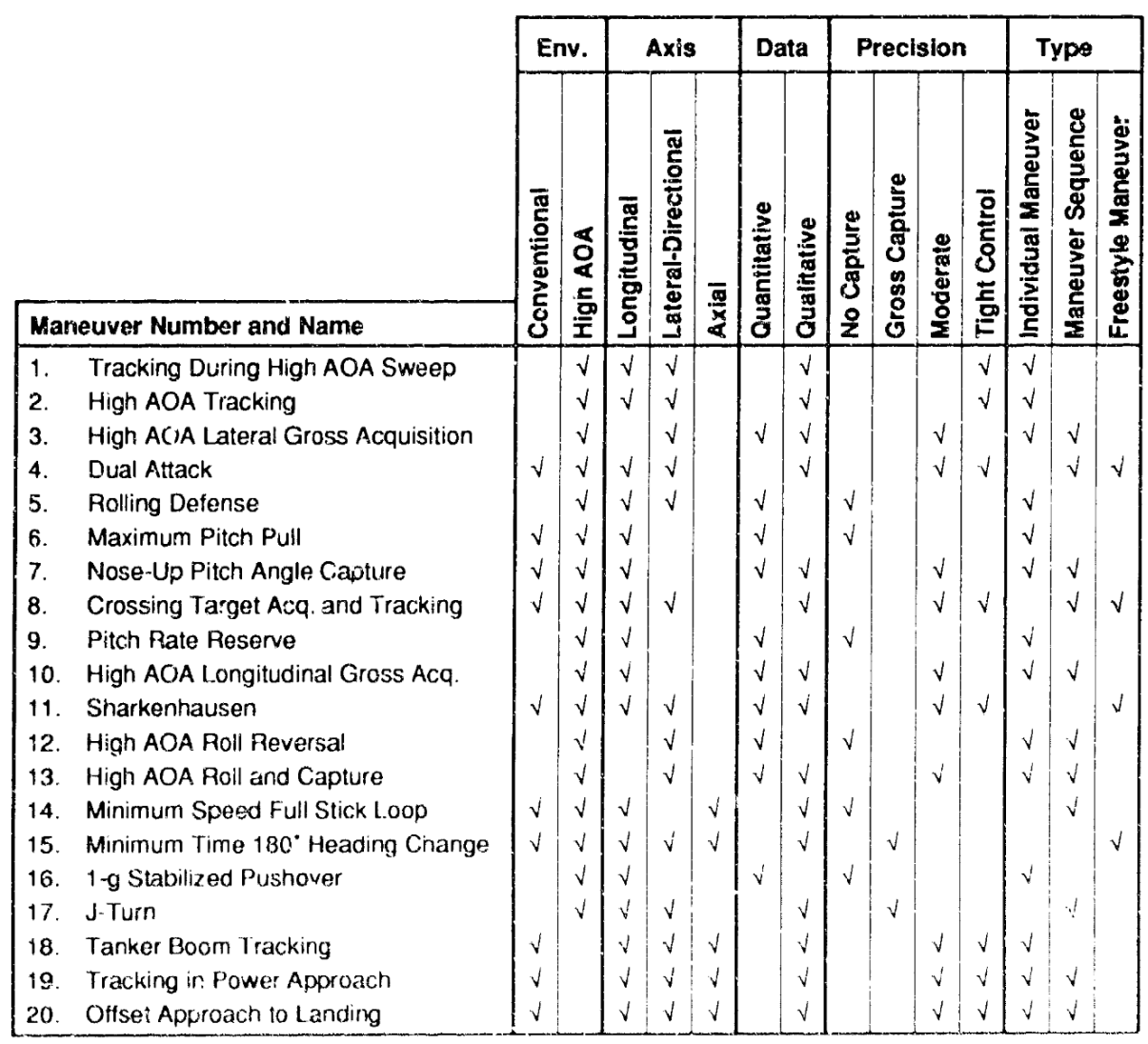

Figure 2.15: Design parameters related to manoeuvres [32]

Wilson et al. [32] show that the most promising manoeuvres to test the longitudinal handling qualities are the ones listed below. A more elaborate explanation of the manoeuvres and why these are selected can be found in the work by Wilson et al. [32].

- Tracking during high angle of attack sweep

- High angle of attack tracking

- Dual attack

- Nose-up pitch angle capture

- Crossing target and track target

- High angle of attack longitudinal gross acquisition

- Sharkenhausen

- Tanker boom tracking

- Offset approach to landing 


\subsection{Simulation models}

To design the variable stability in-flight simulator, some simulation models regarding the Cessna Citation II are required. Firstly, the aircraft simulation model is described. Secondly, the actuator model is introduced.

\subsubsection{Cessna Citation II aircraft simulation model}

A non-linear Cessna Citation model has been developed at Delft University of Technology which is called CitAST, compiled by Borst [33]. It is a stripped version of the Delft University Aircraft Simulation Model and Analysis Tool (DASMAT) and allows to generate offline simulations with the complete six degree of freedom model from the Cessna Citation 500. With CitAST the non-linear model can be trimmed, linearised around a specific operating point, customised to add newly designed controllers and it can also be exported to the Delft University Environment for Communication and Activation (DUECA) environment to use in real-time simulations.

The main disadvantage of the Cessna Citation 500 simulation model is that it does not match the current Cessna Citation II aircraft that is flown in flight tests. Van den Hoek et al. [34] has been working on the development of a new model for the Cessna Citation II, however due to the unavailability of flight test data not all parameters could be estimated. The influence from the trim tabs (small actuator surfaces at the end of the elevator with the purpose of lowering the hinge moment) and the flaps are the main parameters missing in the newer model. The trim tabs are of great influence to the actuator model, which is why application of the newer model is not beneficial. Next to this, the main purpose of this research is to create a variable stability in flight simulator that can match other aircraft's dynamics. For testing concepts it is not necessary to have the actual dynamics of the Cessna Citation II, since these dynamics should be cancelled anyway. Both reasons have lead to the decision of using the CitAST model compiled by Borst for the non-linear aircraft simulator model.

\subsubsection{Actuator model}

A non-linear actuator model for the Cessna Citation II has been developed by Lubbers [35]. Lubbers mainly focused on the pitch and roll motions, developing an accurate model for the hinge moments of the ailerons and the elevator. The unknowns from the models are estimated by a combination of ground and flight tests. His models consist of the following submodels:

- Aileron: Yoke, Cables, Servo and Aileron Sector Assembly, Aileron and Aerodynamic hinge moment.

- Elevator: Column, Cables, Servo, Downspring, Elevator and Aerodynamic hinge moment.

In the tests with pilots by Mirza et al. [3], the actuator model was used to translate the inputs from the pilot to the deflection of the elevator. However it was noticed that some error in the model existed which resulted in a very small elevator deflection. The hinge-moment in the actuator model was modified with a factor of 2.5 , creating a larger deflection. Before testing new variable stability methods in simulation, the model from Lubbers has to be validated with flight test data. This will be presented in Chapter 3. 


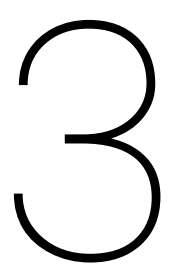

\section{Actuator model validation}

This chapter is dedicated to the actuator model validation. To be able to achieve the variable stability characteristics as required, the actuator model must be validated with flight test data. As explained before, the initial experiment performed by Mirza et al. [3] made use of an adapted version of the actuator model, since the deflections from the original model were not sufficient enough for the pilot to control the aircraft. Firstly, the gathering of the flight data and its pre-processing is described. Secondly, a brief overview of the model is given. Thirdly, the elevator model is checked against the validation data and adapted where necessary. Finally, the same analysis is performed for the aileron model.

\subsection{Flight test data}

Flight test data are acquired from the Cessna Citation II flight tests performed in December 2017 and January 2018. The tests concerned a controller that was also controlling the fly-by-wire system and data is logged with the to be tracked signal and the actual response of the actuator. The following flights were made available as can be seen in Table 3.1.

Table 3.1: Flight test data operating points and remarks

\begin{tabular}{|c|c|c|c|}
\hline Flight data \# & Date & Operating points & Remarks \\
\hline 1 & $14-12-2017$ & $\begin{array}{l}\text { FL170 260knots } \\
\text { FL120 240knots }\end{array}$ & \\
\hline 2 & $18-12-2017$ & FL180 250knots & \\
\hline 3 & $18-12-2017$ & $\begin{array}{l}\text { FL180 260knots } \\
\text { FL180 150knots }\end{array}$ & \\
\hline 4 & $22-12-2017$ & $\begin{array}{l}\text { FL120 240knots } \\
\text { FL120 180knots }\end{array}$ & \\
\hline 5 & $22-12-2017$ & $\begin{array}{l}\text { FL120 240knots } \\
\text { FL140 195knots }\end{array}$ & \\
\hline 6 & $22-12-2017$ & $\begin{array}{l}\text { Varying altitude } \\
\text { Varying speed }\end{array}$ & Climb and descend in relative short datafile \\
\hline 7 & $22-12-2017$ & FL070 280knots & \\
\hline 8 & 06-02-2018 & FL180 210knots & \\
\hline 9 & 07-02-2018 & $\begin{array}{l}\text { FL090 130knots } \\
\text { FL090 175knots }\end{array}$ & Partially stall flight test \\
\hline 10 & 08-02-2018 & FL075 175knots & Partially stall flight test \\
\hline
\end{tabular}

The flight test data are split into two different logging systems. One is the normal FTISlog, which is the log from the Flight Test Instrumentation System of the Cessna Citation II. The second log file is the AppServolog, which logs all the data concerning the fly-by-wire actuator system. The data from the FTIS log can be converted to usable data with the Highly Effective Flight Test Instrumentation GUI (HEFTIG) tool, but support 
for the AppServolog was not yet included.

The first step was to rewrite the HEFTIG tool in such a way that the AppServolog data and the FTISlog data could be combined in one final datafile. This was required, because the model from Lubbers [35] requires inputs from both log files to run the offline simulation for validation. The main problem with combining both log files, was to ensure the timing of both logs matches. Since the logging for both systems does not start at the same time, the datafiles cannot just be laid ontop of each other. Instead, the DUECA time is used as an identifier and the data is resampled using a linear timeseries of the data. Finally the results are combined in one final datafile that can be used as an input for the offline simulation.

The model from Lubbers requires the following inputs as given in Table 3.2. Note that not all the inputs are used to calculate the elevator deflections, some are used for the validation as shown in the remarks. The inputs are generated from the datafile and the response is simulated in Simulink.

Table 3.2: Actuator model inputs Lubbers model

\begin{tabular}{llll}
\hline \hline Input \# & Variable & Unit & Remarks \\
\hline 1 & $\delta_{a_{t}}$ & {$[\mathrm{rad}]$} & Target aileron deflection \\
2 & $\delta_{e_{t}}$ & {$[\mathrm{rad}]$} & Target elevator deflection \\
3 & $\delta_{r_{t}}$ & {$[\mathrm{rad}]$} & Target rudder deflection (not controlled) \\
4 & $h$ & {$[\mathrm{~m}]$} & Altitude \\
5 & $V_{t a s}$ & {$[\mathrm{~m} / \mathrm{s}]$} & True airspeed \\
6 & $\alpha$ & {$[\mathrm{rad}]$} & Angle of attack \\
7 & $\beta$ & {$[\mathrm{rad}]$} & Sideslip angle \\
8 & $a_{z}$ & {$\left[\mathrm{~m} / \mathrm{s}^{2}\right]$} & Normal acceleration \\
9 & $p, q, r$ & {$[\mathrm{rad} / \mathrm{s}]$} & Angular velocities \\
10 & $\delta_{a_{\text {actual }}}$ & {$[\mathrm{rad}]$} & Actual aileron deflection (required for validation) \\
11 & $\delta_{e_{\text {actual }}}$ & {$[\mathrm{rad}]$} & Actual elevator deflection (required for validation) \\
12 & $\delta_{\text {te }}$ & {$[\mathrm{rad}]$} & Actual trim tab deflection elevator (required as input) \\
13 & $I_{e-\text { servo }_{a c t u a l}}$ & {$[\mathrm{~A}]$} & Actual servo current elevator (required for validation) \\
14 & $I_{a-\text { ser }_{\text {actual }}}$ & {$[\mathrm{A}]$} & Actual servo current aileron (required for validation) \\
15 & $V_{e-\text { ser }_{\text {actual }}}$ & {$[\mathrm{V}]$} & Actual servo voltage elevator (required for validation) \\
16 & $V_{a-\text { servo }_{a c t u a l}}[\mathrm{~V}]$ & Actual servo voltage aileron (required for validation) \\
\hline \hline
\end{tabular}

Having pre-processed the flight data, the model can now be described in more detail in the next section. 


\subsection{Model overview}

A brief overview of the implementation of the model from Lubbers is presented in this section. A schematic overview of this model is presented in Figure 3.1. It should be noted that Lubbers only designed the AppServo model. The control system around this model is added at a later stage by Van Paassen at the section Control and Simulation of Delft University of Technology.

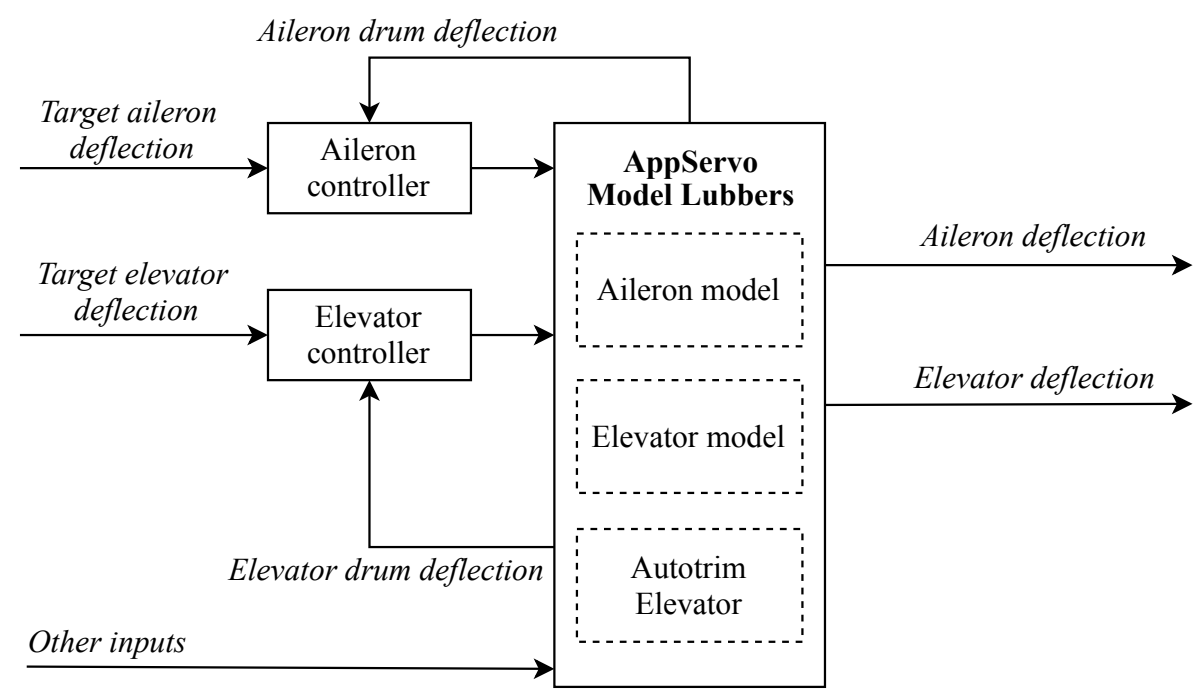

Figure 3.1: Schematic overview of the non-linear actuator model

The model requires the inputs as given in Table 3.2, but to understand the general workings of the model, the target deflections are the most interesting parameters to consider. They are given as input to a controller, with the model having a different controller for the aileron and the elevator. The controllers also have the aileron or elevator drum deflection as feedback input. Using a feedback gain that has been previously designed by Van Paassen, an input is generated for the AppServo model to achieve the desired target deflection. The controller also has the option to apply a feedforward gain to the target deflections, but the initial value of this feedforward gain is equal to one.

The full inner workings of the AppServo model are described in the work from Lubbers and consist mainly of the non-linear aileron model and the non-linear elevator model. The autotrim model for the elevator is added at a later stage by Van Paassen. The main output of the model consist of the aileron deflection and the elevator deflection. These can be compared to the actual flight data to validate the model. It should be noted that the autotrim model is not used in this validation procedure, since the flight data also have the deflection of the trim tabs available. These trim tab data are used as an input for the AppServo elevator model instead of the autotrim model.

The full schematic overview of the non-linear actuators can be found in Lubbers [35], but an example of the elevator is given in Figure 3.2. The output from the elevator controller from Figure 3.1 is given as an input to the autopilot servo, which controls the drum deflection of the elevator. This drum deflection is in its turn used by the elevator controller to ensure the model follows the requested target deflection. All the blocks in the model are based on the actual physical system in the Cessna Citation II. The schematic shows the complexity of the system, since everything depends on each other. Lubbers performed a parameter estimation on the unknown aerodynamic coefficients that create the hinge moment $H_{e}$. Figure 3.2 shows only the schematic of the elevator, but he performed a similar analysis for the aileron fly by wire system. The different components of the hinge moment of the aileron system $H_{a}$ are also estimated using a parameter estimation method. 


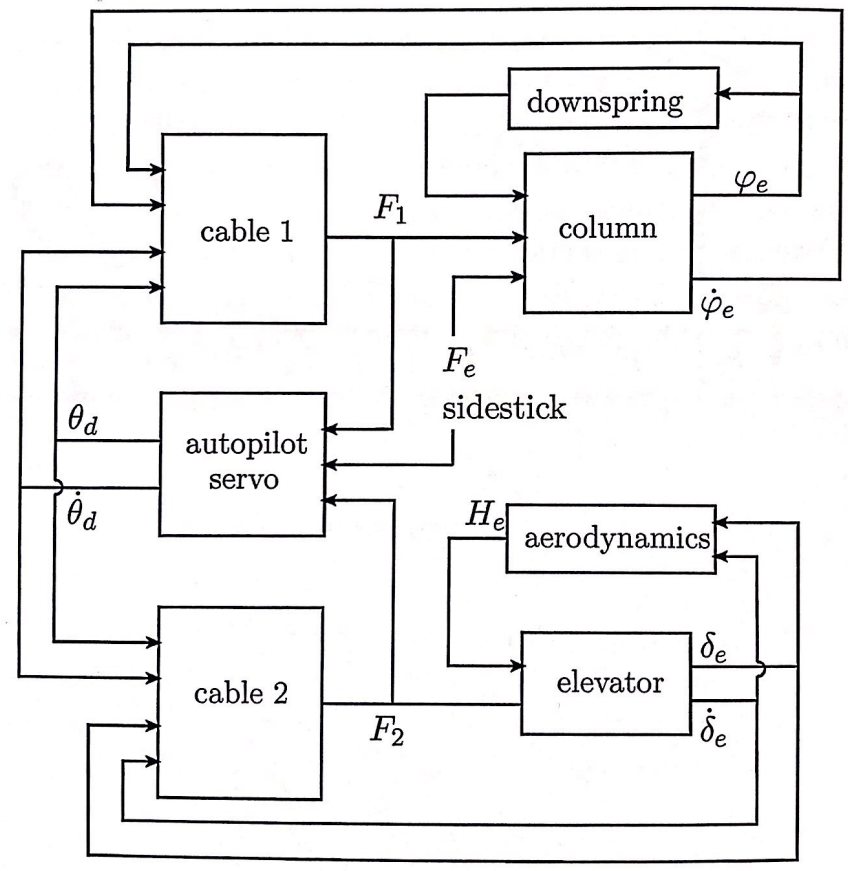

Figure 3.2: Schematic overview of the non-linear elevator model [35] 


\subsection{Elevator}

Using the processed flight data and the model presented in the previous section, offline simulations could be run to validate the elevator model. Flight data number 8 was chosen for the initial checks, since it provides many short period measurements where the fly-by-wire system is used. The initial response from the model can be seen in Figure 3.3. In the top graph the deflection from the actuator model from Lubbers and the actual deflection are depicted. In the lower graph the servo current is compared between the model and the actual flight data. This current is calculated within the autopilot servo block as depicted in Figure 3.2, based on a model of the servo motor.

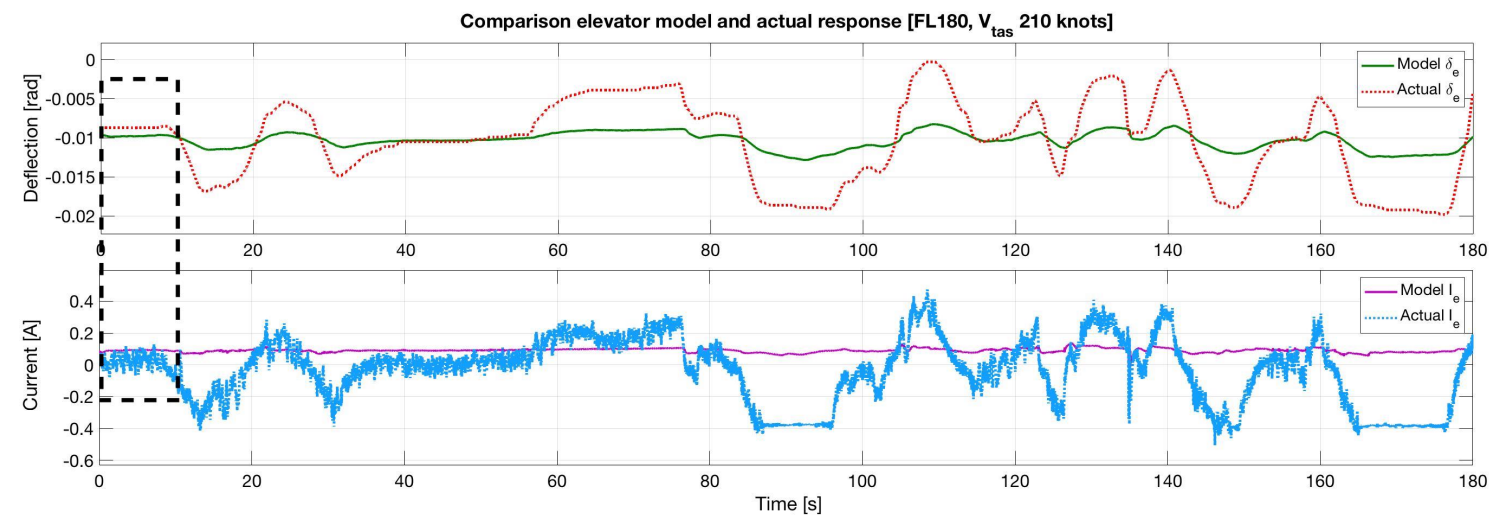

Figure 3.3: Comparison elevator model and actual response from flight test

One can clearly see the issue described earlier by Mirza in his experiment. The deflections from the model are minimal compared to the actual deflections experienced in the flight. Another interesting point to note is the deviation from the initial deflection. The first 10 seconds (within the black dotted box in Figure 3.3), there is no pilot input yet to the model, but it deviates quickly from its initial condition. This is an indicator for an error in the hinge moment calculation.

After researching the specific components that created the hinge moment, it was concluded that the error could not be dedicated to one specific parameter in this model by Lubbers. If one would want to determine this error, a complete new parameter estimation would have to be performed, which is not the scope of this research. That is why it was decided to match the initial response of the model by multiplying the hinge moment coefficient with a gain of 0.6 . This gain was determined by testing different gains in the range of 0.2 to 1.8 on all different operating points as given in the flight data. The gain of 0.6 showed best performance with the lowest error. Note that in Mirza's experiment the hinge moment coefficient was changed with an arbitrary gain of 0.4 , so this was already a good estimation. The result from this gain change can be seen in Figure 3.4. Here, the initial condition is tracked much better as compared to Figure 3.3 as depicted within the black dotted box.

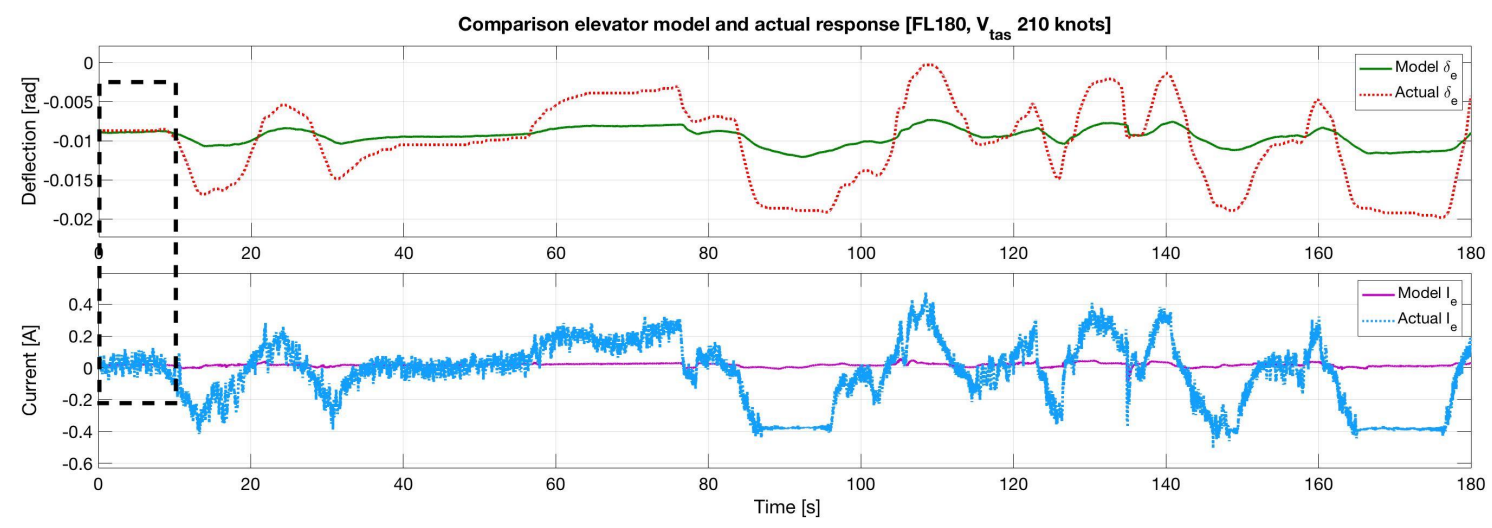

Figure 3.4: Comparison elevator model and actual response from flight test with updated hinge moment 
However, the remainder of the deflection from the elevator model was still not matching the actual deflection from the flight test. It was found that the compiled model used by Mirza was actually controlling the deflections of the drums $\theta_{d}$ inside the fly-by-wire system, instead of the actual deflection of the actuators $\left(\delta_{e}\right.$ and $\delta_{a}$ ). The input from the controllers as shown in Figure 3.1 is the target actuator deflection, but the output is controlling the deflection of the autopilot servo drum. This was decided by Van Paassen, since the non-linear behaviour of the cables and the hinge moment make it difficult to control the deflection properly without introducing oscillations. It is easier to control the drum deflection because most non-linearities occur after the control of the drum, in the cables and the aerodynamics subsystem as given in Figure 3.2.

To correct for the mismatch between the target actuator deflection as an input and the controlled drums by the actuator controllers, the requested target deflection requires a feedforward gain in the elevator controller. After linearisation of the model from Lubbers, it was found that a feedforward gain of 6 would ensure better matching capabilities of the total elevator model. Again this was tested on different operating points. The result can be seen in Figure 3.5.

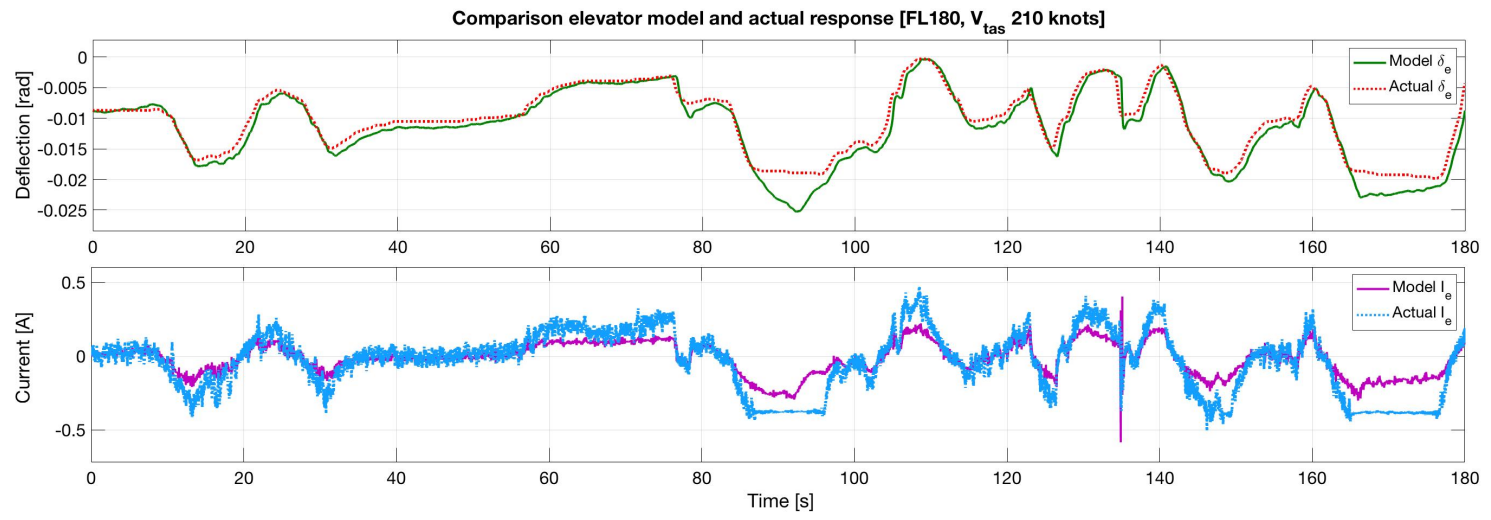

Figure 3.5: Comparison elevator model and actual response from flight test with updated hinge moment and feedforward gain

Now the only remaining issue is the saturation of the elevator servo, or in other words, the limiting current after which the servo drum cannot deflect any further. In Figure 3.5 it can be seen that this saturation occurs around 90 seconds and around 170 seconds. Because of the updated hinge moment and feedforward gain, the modelled current in the servo is not matching the actual current in the servo anymore. Instead of trying to match the current, which was found to be very difficult for all different operating points, it was decided to change the resistance in the autopilot servo motor model to achieve the limiting current at the same timing as during the flight tests. The $R h t_{e}$ and $R l t_{e}$ were changed to $2.01 e 6$ and $4.19 e 5$, respectively, in the FBWData- $\nu 3$ file. These are parameters that influence the current limiting response within the autopilot servo block from Figure 3.2. All other parameter values are kept the same as presented in the work by Lubbers. This gives the result as given in Figure 3.6.

The limit current is detected at the same time, but during the saturation the elevator deflection of the model still has some error compared to the actual elevator deflection. This can be explained by to the parameter estimation of the hinge moment from Lubbers that is not valid anymore when the servo is saturated. However, it has been decided not to further investigate the effects of this servo saturation on the hinge moment parameters, since the performance is considered to be sufficiently accurate for the purposes of this thesis. 

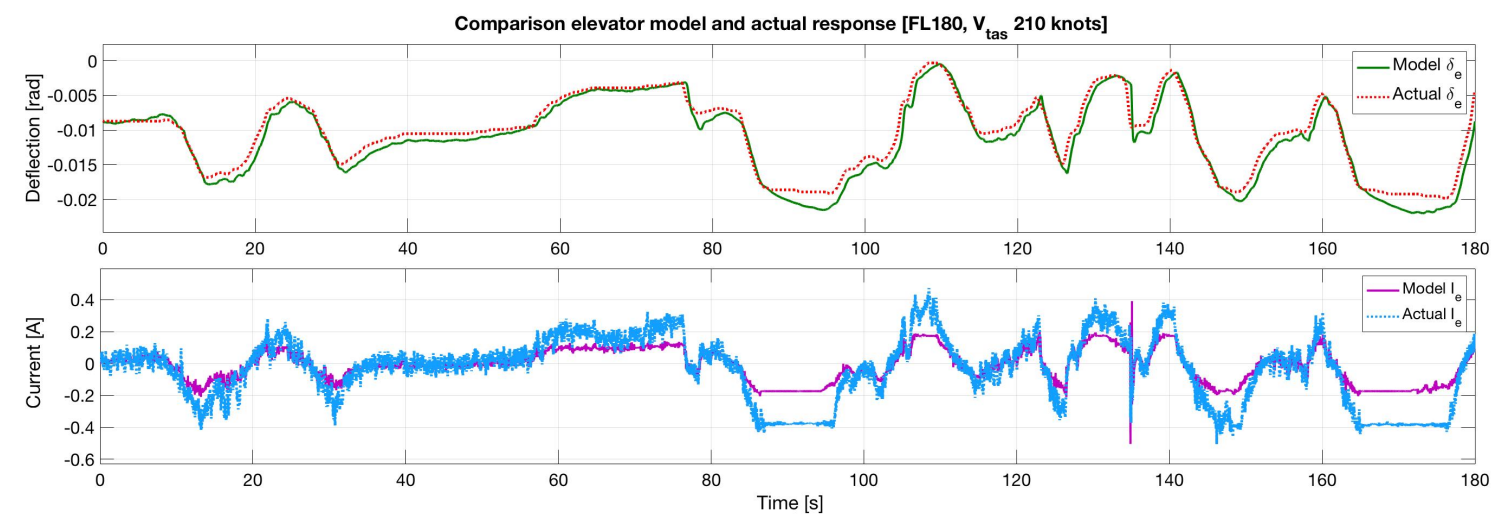

Figure 3.6: Comparison elevator model and actual response from flight test with updated hinge moment, feedforward gain and corrected limit detection

One of the other flight conditions that are tested is shown in Figure 3.7. This flight condition is chosen, since it consists of an induced oscillation on the elevator. Even in these different flight conditions, the newly updated model matches the actual deflection well. Next the aileron model can be validated.

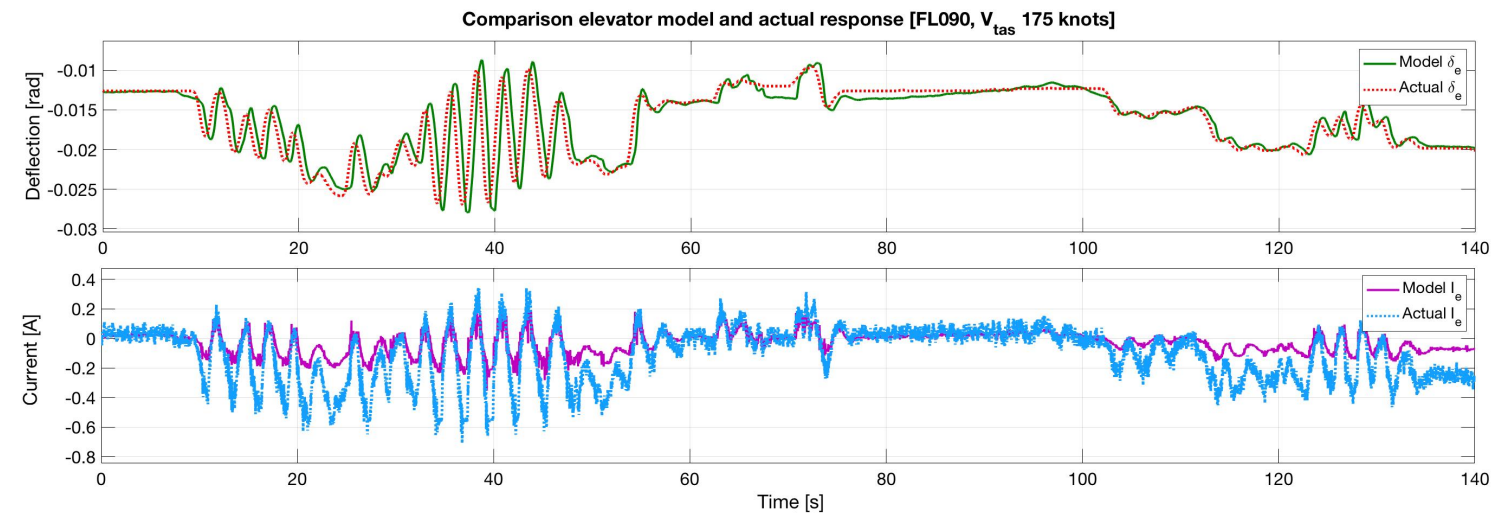

Figure 3.7: Comparison corrected elevator model and actual response from flight test for different flight condition 


\subsection{Aileron}

With an improved elevator model, the aileron model can be investigated in more detail. The same procedure as for the elevator is followed. The initial response from the aileron model can be seen in Figure 3.8. Here, the initial condition of the aileron position seems to be followed correctly, contradicting the initial response of the elevator (shown within the black dotted box). This would imply that the hinge moment calculation of the aileron is without error. This has been tested for different operating conditions and was found to be true.

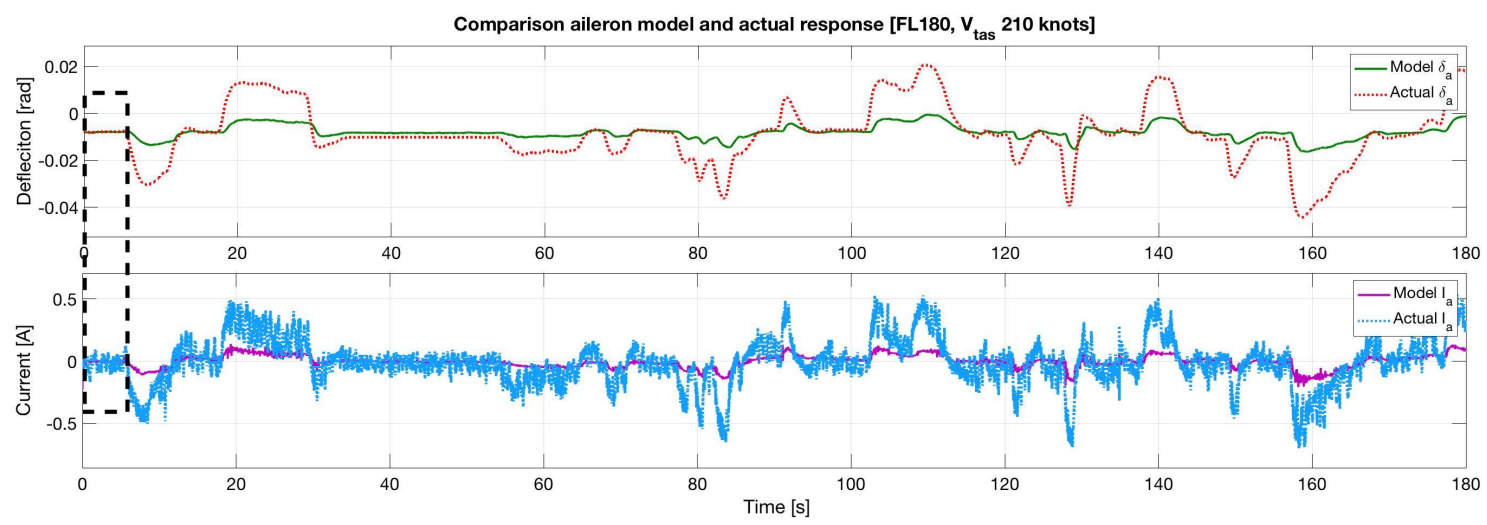

Figure 3.8: Comparison aileron model and actual response from flight test

However, the response from the aileron model is not matching the actual deflection from the flight test. This can be explained by the model controlling the aileron drum servo instead of the actual aileron deflection, which is the same issue as previously presented for the elevator. That is why a feedforward gain has to be applied on the target aileron deflection in the aileron controller. After linearisation of the model from Lubbers it was found that a feedforward gain of 4.2 would allow for the best matching results. This is depicted in Figure 3.9, where the aileron model is now matching the actual deflection. Since no changes are made to the aileron hinge moment model, it is expected that the limit cases for the aileron servo saturation will be correct.

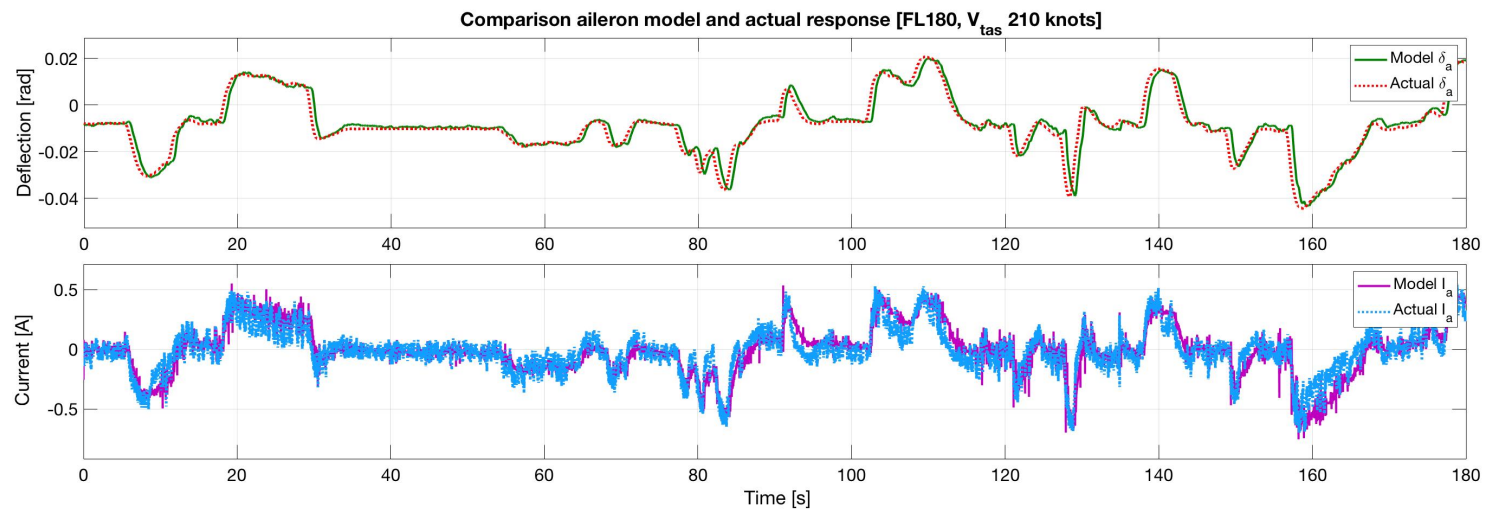

Figure 3.9: Comparison aileron model and actual response from flight test with updated feedforward gain

Not much data was available where the aileron servo is reaching its saturation limit, but one case was found in flight data file number 10. The results are depicted in Figure 3.10. One can clearly note the limited saturation at 70 and 270 seconds. For the aileron model, a small underestimation can be seen when the servo is saturated. This can also be explained by the parameter estimation from Lubbers that is not valid anymore at the limits of the servo current. The performance is considered to be sufficient for the purposes of this thesis. 


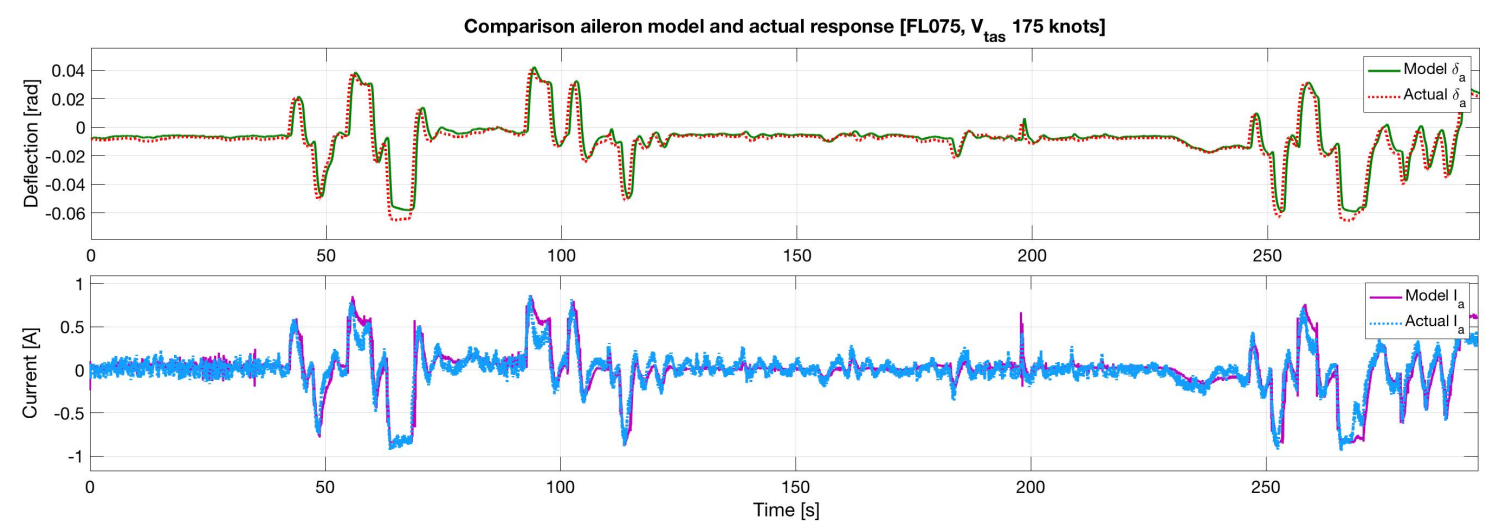

Figure 3.10: Comparison corrected aileron model and actual response from flight test for servo current saturation

Another operation point can be found in Figure 3.11. It also shows good performance of estimating the actual aileron deflection. One point of interest is the higher oscillatory behaviour in the aileron servo data in both Figures 3.10 and 3.11 compared to the model. This is something that will not influence the results of the model estimation significantly, but is worthwhile noting.
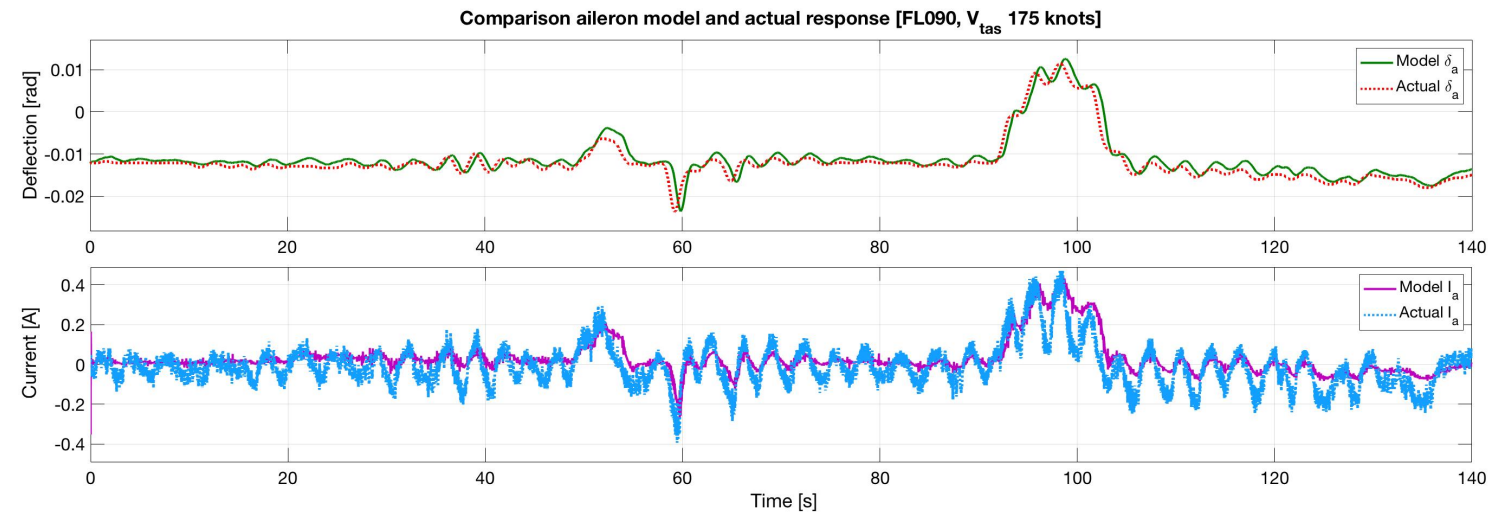

Figure 3.11: Comparison corrected aileron model and actual response from flight test for different flight condition

The actuator model is now validated for the different conditions presented in this chapter. It can be incorporated in the actual model of the aircraft to investigate the variable stability limits. This will be researched in the next chapter. 



\section{4}

\section{Variable stability limits}

This chapter is dedicated to finding the variable stability limits of the (non-linear) dynamic inversion method. Firstly, the methodology is discussed. Secondly, the integration of the actuator and aircraft model is described. Thirdly, the dynamic inversion limits are investigated. Fourthly, the maximum elevator deflection is researched and finally the optimum operating point in the flight envelope is determined.

\subsection{Methodology}

The variable stability limits can be split in two main components, being the fly-by-wire actuator limits and the incremental non-linear dynamic inversion limits. Combined they should give an indication of the operating point in the flight envelope which provides the most opportunities regarding the dynamic inversion.

The variable stability limit is investigated in the longitudinal motion. The longitudinal motion is chosen over the roll motion, because the elevator has different initial deflections in different flight conditions to achieve straight and steady flight. This essentially means that the maximum elevator deflection that can be achieved will vary dependent on the initial position. The initial deflection from the ailerons will always be zero in this case (except when wind or disturbances are introduced), which makes it a less interesting parameter to look at. Next to this, the longitudinal motion is of higher interest when determining the handling qualities.

Following basic flight dynamics, the longitudinal motion can be described by Equations 4.1-4.2, where $M$ is the moment, $M_{\delta_{e}}$ is the moment due to the elevator deflection, $I_{y}$ is the inertia around the y axis, $\delta_{e_{c m d}}$ is the commanded elevator deflection, $u$ is the control input and $\dot{q}_{c m d}$ is the commanded pitch acceleration. The commanded pitch acceleration represents the pitch acceleration that results from the model that should be followed by the system.

$$
\begin{gathered}
\Delta M=M_{\delta_{e}} \cdot \Delta \delta_{e_{c m d}}=I_{y} \cdot \Delta \dot{q}_{c m d} \\
\Delta u=\Delta \delta_{e_{c m d}}=\frac{I_{y}}{M_{\delta_{e}}} \cdot \Delta \dot{q}_{c m d}
\end{gathered}
$$

Rewriting Equation 4.2 gives us Equation 4.3 which can be used for the determination of the variable stability limits.

$$
\Delta \delta_{e_{c m d}} \cdot \frac{M_{\delta_{e}}}{I_{y}}=\Delta \dot{q}_{c m d}
$$

In this equation, $\Delta \dot{q}_{c m d}$ is the variable stability system limitation. This is the maximum pitch acceleration that can be followed by the system. It consists of the longitudinal moment effectiveness parameter $\frac{M_{\delta_{e}}}{I_{y}}$ which is the dynamic inversion limit. This parameter should be maximised to achieve the best performance. The fly-by-wire limitations arise from the maximum $\Delta \delta_{e_{c m d}}$. This can be investigated by finding the maximum deflections of the elevator at different initial conditions. However, firstly the actuator and aircraft model will have to be integrated and trimmed. 


\subsection{Model integration and trimming}

To find the variable stability limits, the validated non-linear actuator model has to be integrated with the non-linear CitAST model. To provide a clearer understanding of this integration, an overview is presented in this section. Figure 4.1 shows the basic CitAST model as compiled by Borst [33].

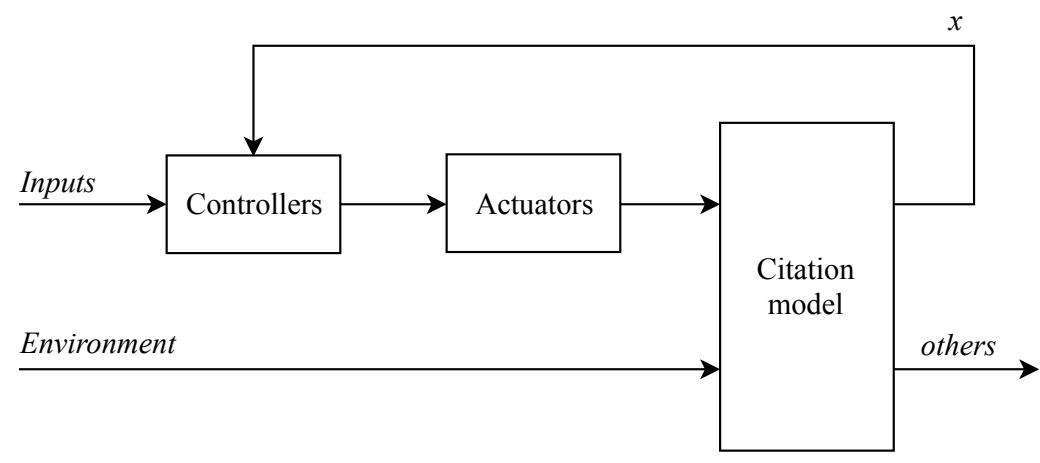

Figure 4.1: Schematic overview of the CitAST model for the Cessna Citation 500

Two main inputs exist in the CitAST model, being the pilot input to the controllers block and environmental input to the Citation model block. The environmental inputs consist of turbulence and terrain elevation. They are not relevant when determining the variable stability limits, which is why these inputs are equal to zero. The pilot input is as defined in Table 4.1 .

Table 4.1: Description of pilot inputs to the CitAST model

\begin{tabular}{llll}
\hline \hline Input \# & Variable & Unit & Remarks \\
\hline \hline 1 & $\delta_{e}$ & {$[\mathrm{rad}]$} & Elevator deflection \\
2 & $\delta_{a}$ & {$[\mathrm{rad}]$} & Aileron deflection \\
3 & $\delta_{r}$ & {$[\mathrm{rad}]$} & Rudder deflection \\
4 & $\delta_{t e}$ & {$[\mathrm{rad}]$} & Elevator trim tab deflection \\
5 & $\delta_{t a}$ & {$[\mathrm{rad}]$} & Aileron trim tab deflection \\
6 & $\delta_{t r}$ & {$[\mathrm{rad}]$} & Rudder trim tab deflection \\
7 & $\delta_{f}$ & {$[\mathrm{rad}]$} & Flaps deflection \\
8 & gear & {$[-]$} & Gear up or down $(0$ or 1$)$ \\
9 & pla1 & {$[-]$} & Power lever 1 (between 0 and 1$)$ \\
10 & pla2 & {$[-]$} & Power lever 2 (between 0 and 1$)$ \\
11 & brakel & {$[-]$} & Left brake (between 0 and 1$)$ \\
12 & braker & {$[-]$} & Right brake (between 0 and 1$)$ \\
13 & nws & {$[\mathrm{rad}]$} & Nose wheel steering \\
14 & $y d$ & {$[-]$} & Yaw damper on or off $(1$ or 0$)$ \\
\hline \hline
\end{tabular}


This complete model consists of three main blocks. They are shortly described below:

- Controllers: The controllers block consists of two controllers, being the yaw damper and the auto throttle. State feedback from the Citation model block is used by these controllers. They modify some of the inputs given by the pilot as described in Table 4.1.

- Actuators: Within the actuator block, some of the inputs are modified with a first order lag system. These lag systems are chosen in such a way that they match the actual response of the actuators as closely as possible. However, since the actual actuators are higher order non-linear systems this approximation is not accurate. Next to these lag systems, some limits are added to match the limits of the actuator deflections of the real aircraft. A schematic overview of the actuators block can be found in Figure 4.2.

- Citation model: This block consists the compiled version of the DASMAT model of the Cessna Citation 500. It requires a trim file with a predetermined initial condition as an input which can be generated by the tools supplied in the compiled package from Borst [33].

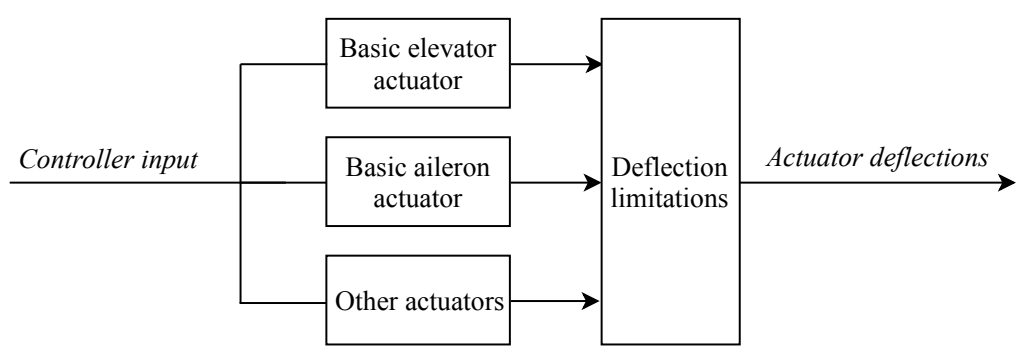

Figure 4.2: Schematic overview of the Actuators block

The modified input from the actuators is given as an input to the Citation model block. From the Citation model the state vector is acquired and fed back into the controllers block. There are several other outputs available as described by Borst [33].

Now the non-linear actuator model described in Section 3.2 should be introduced within the actuators block. The first order lag filters for the elevator and aileron can be replaced by the non-linear model (described as basic elevator/aileron actuator in Figure 4.2). First it is required to modify the model and add the state feedback and normal acceleration as an input to the actuators block, since they are required by the nonlinear model from Lubbers. The modified schematic overview is depicted in Figure 4.3.

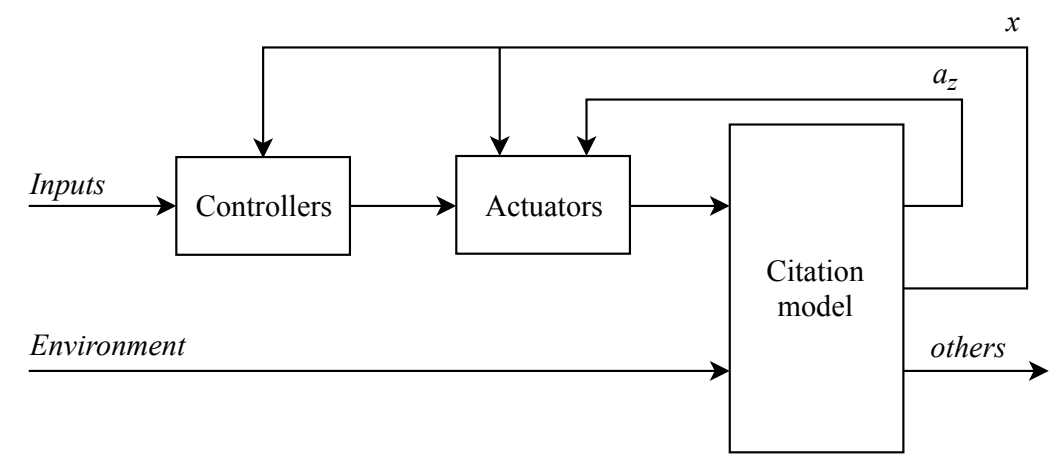

Figure 4.3: Modified schematic overview of the CitAST model for the Cessna Citation 500 
This gives us all required inputs to introduce the non-linear model in the actuators block. The result of this is shown in Figure 4.4. The non-linear actuator model and model switcher blocks are introduced. The nonlinear actuator model block consists of the model presented in Figure 3.1. The model switcher block is simply a switch, where the user of the simulation can decide if the non-linear model or the first order lag model should be used to generate the actuator deflections for the aileron and elevator. These actuator deflections are in its turn introduced to the Citation model block.

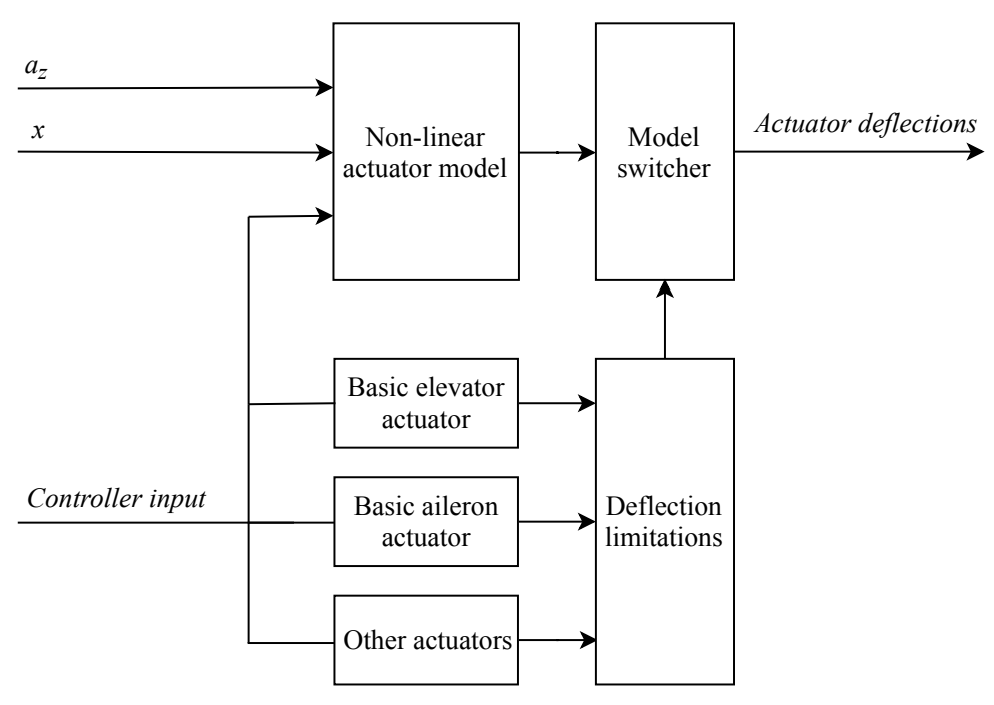

Figure 4.4: Modified schematic overview of the Actuators block

After completing the integration, the system has to be trimmed for the different flight conditions that are of interest. It was decided to use the same flight conditions as Mirza, for consistency within the overall research. The following parameters are combined to find sixteen different initial conditions:

- Height: 2000, 4000, 6000 and $8000[\mathrm{~m}]$

- True airspeed: $90,110,130$ and $150[\mathrm{~m} / \mathrm{s}]$

The combined model of the aircraft and the actuator has to be trimmed for straight and symmetric flight in these conditions. This should be achieved with the minimal hinge moment, so the maximum elevator deflection with the servo's can be reached. The CitAST model does not include the hinge moment, which means it cannot be used to trim the model to the lowest hinge moment possible at a specific initial condition. This is solved by creating an iterative loop between the CitAST trimming tool and the actuator model from Lubbers. This iterative loop is depicted in Figure 4.5.

Firstly, the CitAST model is trimmed for straight symmetric flight at the requested initial condition. This gives a specific elevator deflection as a result. This deflection is used as an input in Lubbers' model to calculate the hinge moment. Now the trim tab deflection can be calculated that minimises the hinge moment. This trim tab deflection is in turn used in the CitAST trimming tool to re-trim the aircraft at the same initial conditions. Because a trim tab angle is introduced, the elevator deflection required is changed from the first iteration. This newly determined elevator deflection is again used in Lubbers' model to determine a new trim tab deflection. This iterative process is continued until no significant changes occur anymore in the deflection of the elevator and the trim tab. 


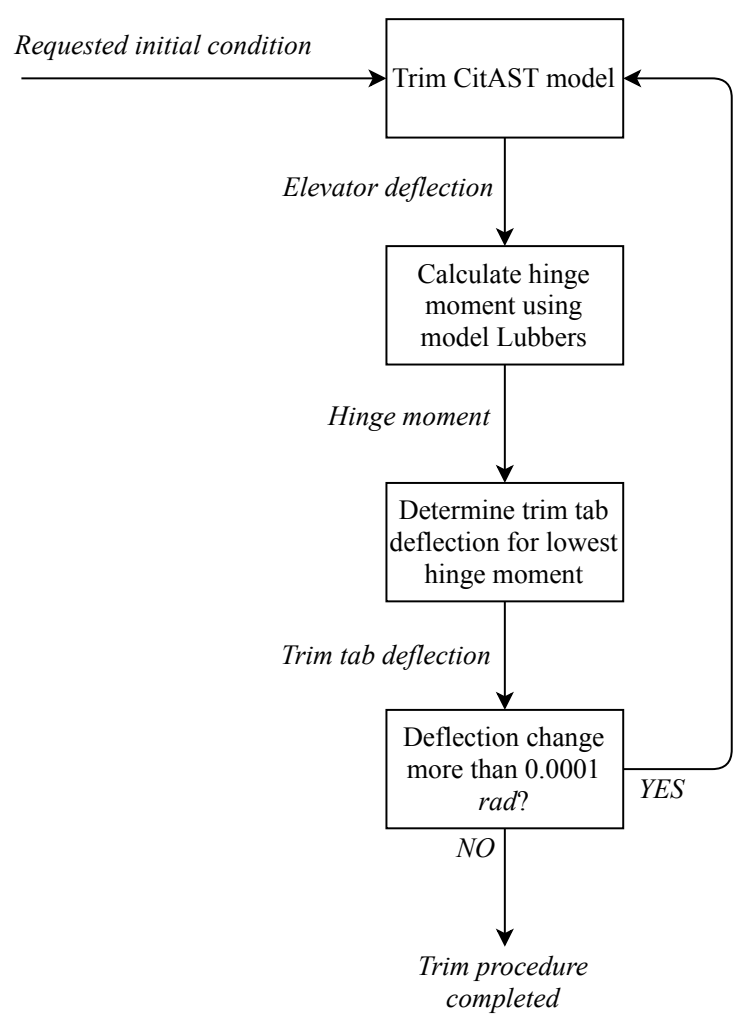

Figure 4.5: Schematic overview of the iterative trim procedure

With an integrated model that is trimmed to the earlier defined initial conditions, the actual variable stability limits can be investigated. We start with the dynamic inversion limits.

\subsection{Dynamic inversion limits}

The dynamic inversion limit is determined by investigating the longitudinal moment effectiveness parameter $\frac{M_{d e}}{I_{y}}$. This parameter can be expanded to Equation 4.4 according to Nelson [5], where $\rho$ is the air density, $V_{t a s}$ is the true airspeed, $S$ is the surface area of the wing, $c$ is the length of the chord and $C_{m_{\delta_{e}}}$ is the moment coefficient due to the deflection of the elevator:

$$
\frac{M_{\delta_{e}}}{I_{y}}=\frac{0.5 \rho V_{T A S} S \bar{c} C_{m_{\delta_{e}}}}{I_{y}^{2}}
$$

As can be seen from Equation 4.4, not all parameters can be influenced to maximise the longitudinal moment effectiveness. Inertia, surface area and the chord are physical parameters of the aircraft itself, which cannot be changed by changing the initial condition. The two main parameters that can be changed are the true airspeed and the density by changing the altitude of flight. The moment coefficient is also investigated by looking at the non-linear CitAST model, to investigate if its parameter composition can be influenced by changing the initial condition. It was found that the moment coefficient depends on the parameters as displayed in Equation 4.5, where $M$ is the Mach number, $V_{i a s}$ is the indicated airspeed, $\alpha$ is the angle of attack and $\delta_{\text {flaps }}$ is the deflection of the flaps:

$$
C_{m_{\delta_{e}}}=\left(f u n(\alpha, M)+f u n\left(\alpha, \delta_{\text {flaps }}\right)\right) \cdot f u n\left(\alpha, \delta_{\text {flaps }}\right) \cdot f u n\left(V_{I A S}\right)
$$

The first parameter in Equation 4.5 (from left to right) is a function of the Mach number and the angle of attack. The value of this parameter can be found in Figure 4.6. It can be seen that the parameter seems dependent on the Mach number, but it only changes with the angle of attack. The angle of attack is something that cannot be influenced when the aircraft is trimmed to straight and steady flight, so the longitudinal moment effectiveness cannot be maximised further using this information. 


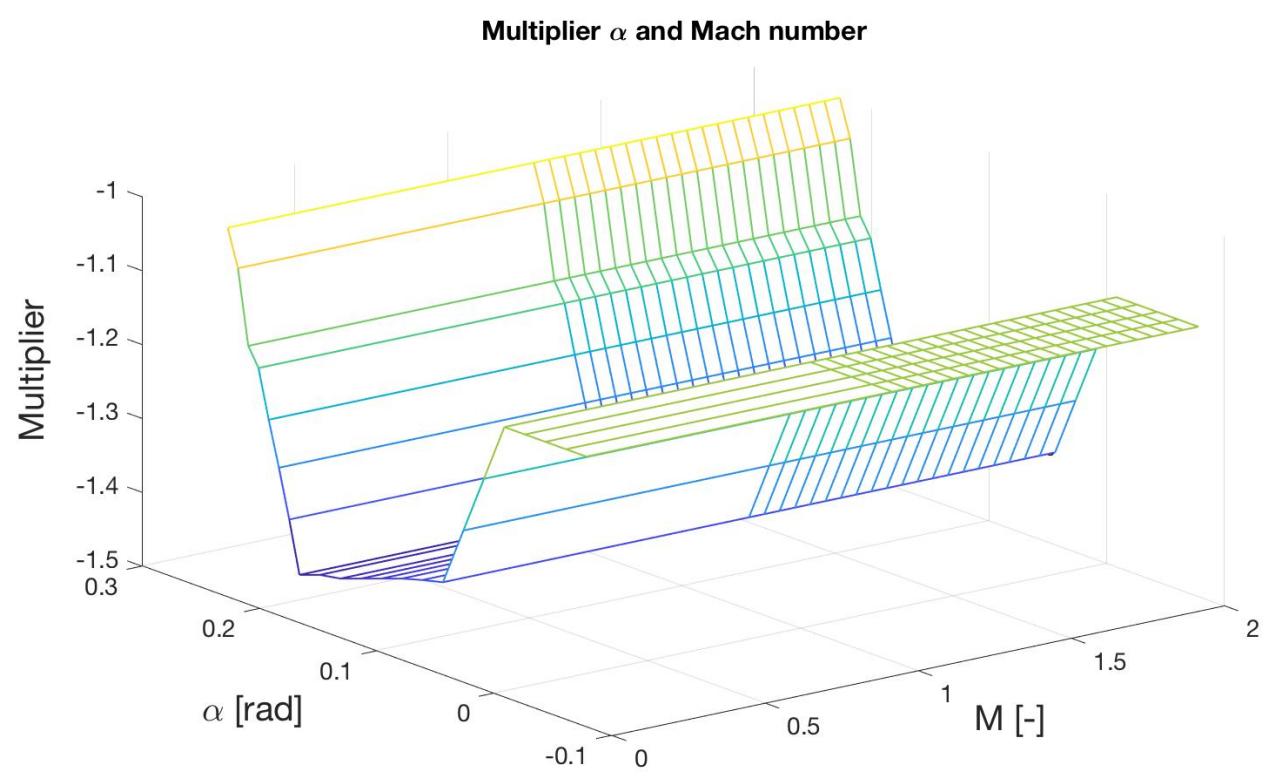

Figure 4.6: Multiplier parameter from CitAST model as a function of angle of attack and Mach number

The second parameter in Equation 4.5 is a function of the angle of attack and the flap deflection. The value of this multiplying parameter can be found in Figure 4.7. It can be seen that the value of this parameter is always zero when the flaps are not extended. During normal operation of the aircraft and especially when flying the variable stability system, flaps would not be used. That is why this parameter will also not influence the moment coefficient.

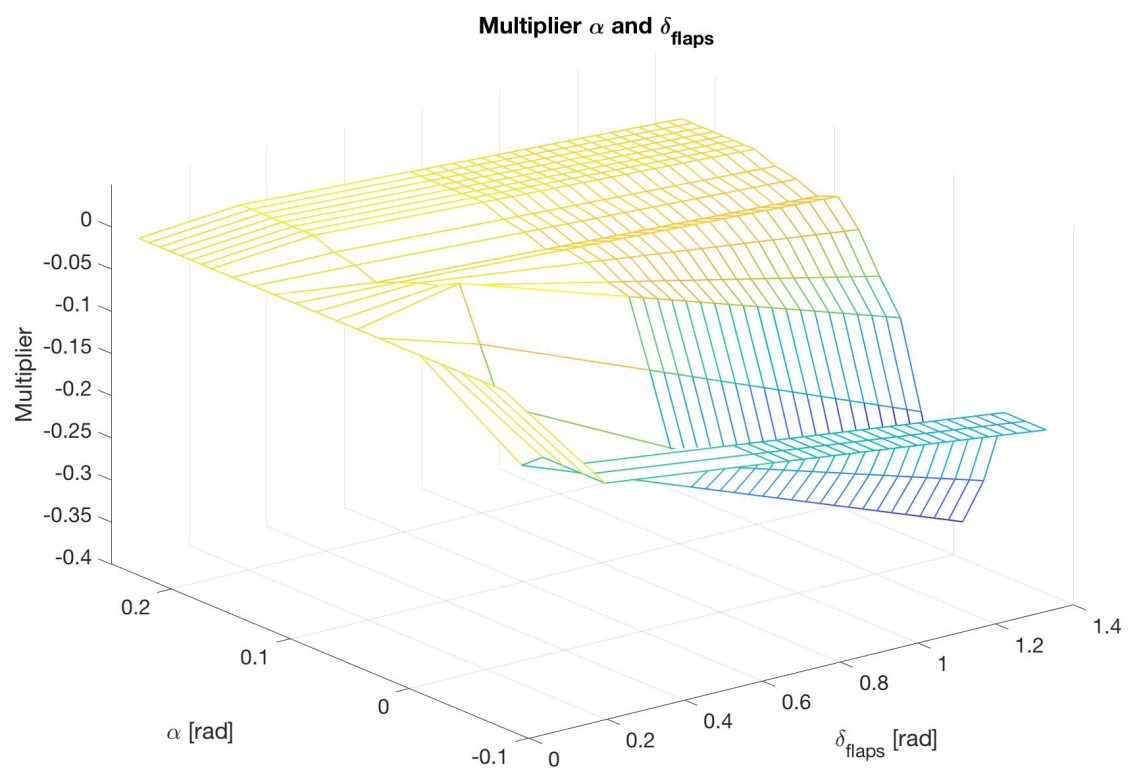

Figure 4.7: Multiplier parameter from CitAST model as a function of angle of attack and flap deflection

The third parameter in Equation 4.5 is also a parameter that is a function of the angle of attack and the flap deflection. The value of this multiplier can be seen in Figure 4.8. This parameter seems to be a correcting parameter for cases with low angle of attack and high flap deflection. In nominal operations this will not be influencing the moment coefficient significantly, since in all the other cases it is equal to one. 


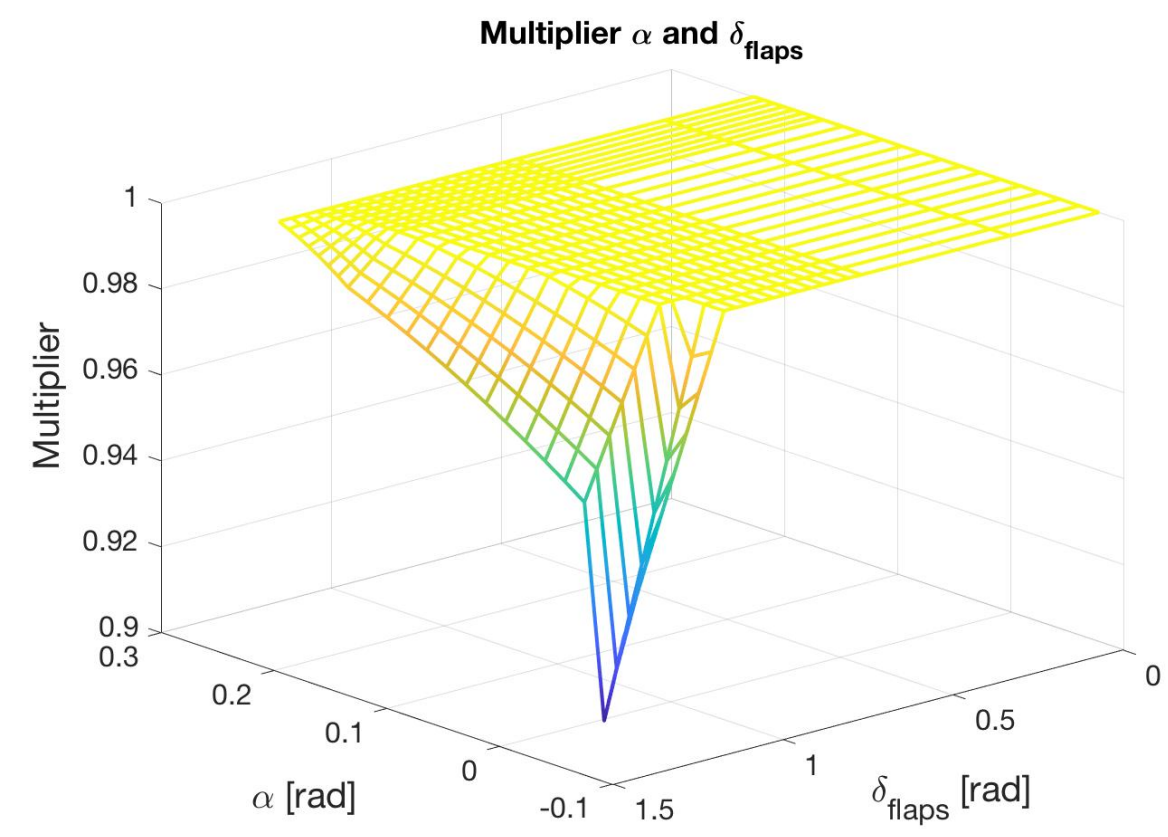

Figure 4.8: Second multiplier parameter from CitAST model as a function of angle of attack and flap deflection

The final parameter in Equation 4.5 is the influence of the indicated airspeed. This is illustrated in Figure 4.9. The multiplier is only limiting the moment coefficient at low indicated airspeed, since the elevator cannot generate a moment when there is no sufficient airflow over the elevator. In nominal flight conditions this will also not influence the moment coefficient.

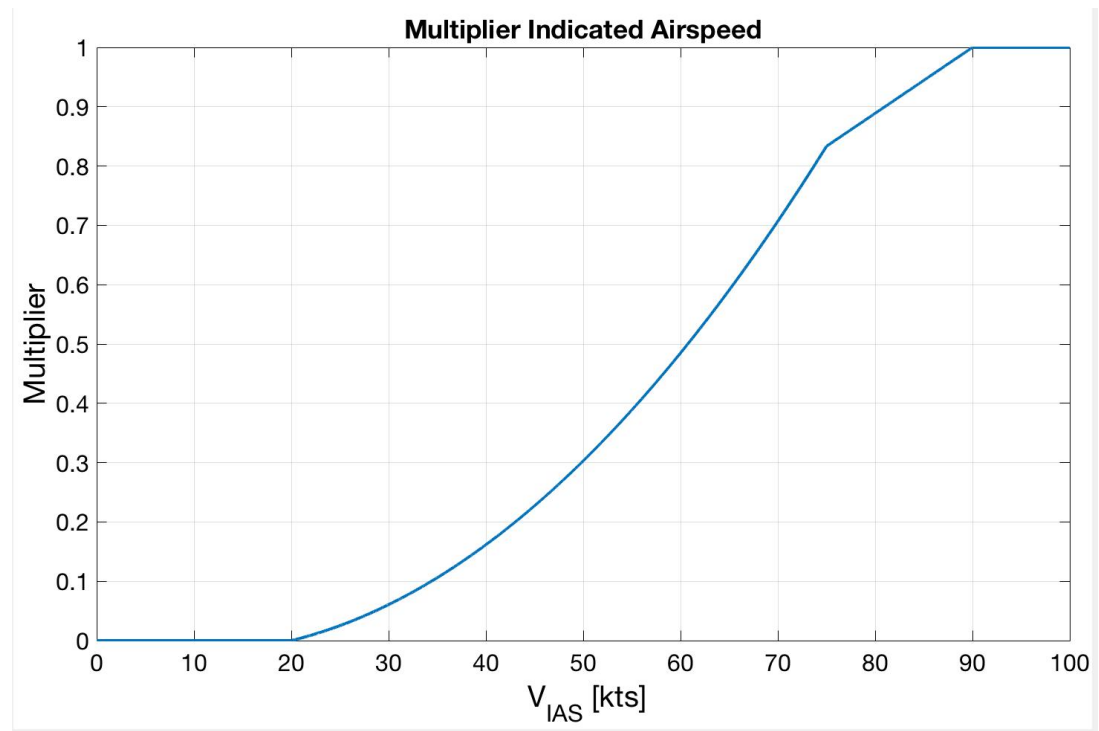

Figure 4.9: Multiplier parameter from CitAST model as a function of indicated airspeed

Now it can be concluded that the only parameters that will mainly be influencing the longitudinal moment effectiveness are the air density and the true airspeed. Running the integrated model at the trimmed initial conditions, the longitudinal moment effectiveness can be calculated. The result of this can be found in Figure 4.10. If the circle is larger compared to others, the relative longitudinal moment effectiveness is also higher at this initial condition. As expected, the highest values are achieved at low altitudes (high density) and high speeds. The actual values of this longitudinal moment effectiveness can be found in Appendix A. 


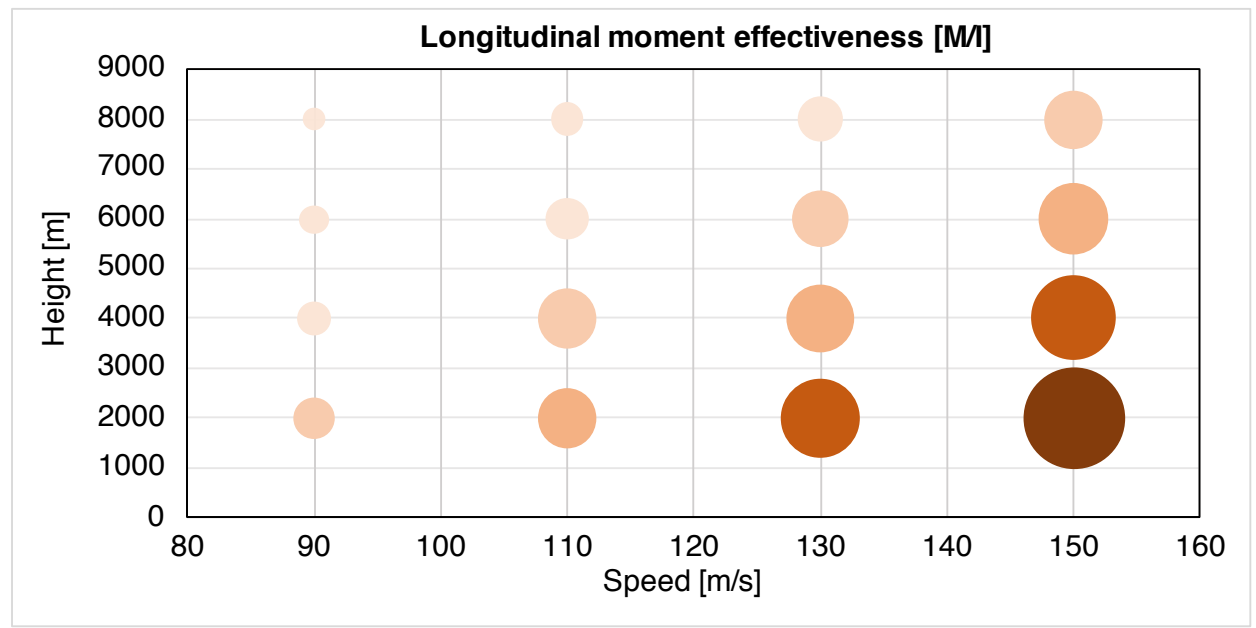

Figure 4.10: Longitudinal moment effectiveness compared for different initial conditions

\subsection{Elevator deflection limits}

Having determined the first part of the variable stability limits, the maximum elevator deflection should be investigated. The model from Lubbers is used combined with the aircraft model to find the saturation of the elevator deflection. One can imagine that this deflection is lower when flying higher speeds. Next to this, when the density is lower, the aerodynamic forces on the elevator will be lower as well. This would increase the possible deflection. These hypotheses are confirmed when we look in Figure 4.11.

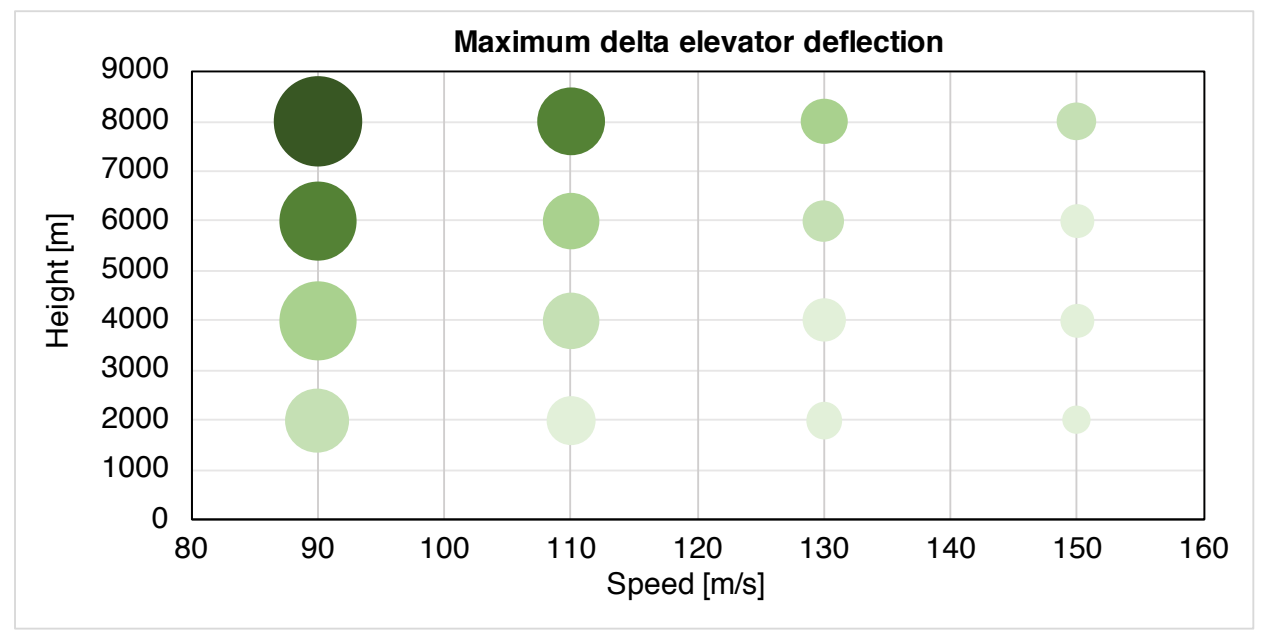

Figure 4.11: Maximum elevator deflection compared for different initial conditions

The maximum elevator deflection at 8000 meters with a true airspeed of 90 meters per seconds gives a deflection of around 1 degrees. This does not seem like a large deflection, but can still be sufficient in performing different types of manoeuvres. This will be confirmed in Chapter 5. 


\subsection{Operating points in flight envelope}

Both contributing factors to the variable stability limits in the flight envelope are determined. Now they can be combined to find the most promising operating point with respect to commanded pitch acceleration. They are combined as presented earlier according to Equation 4.5 and the results can be found in Figure 4.12.

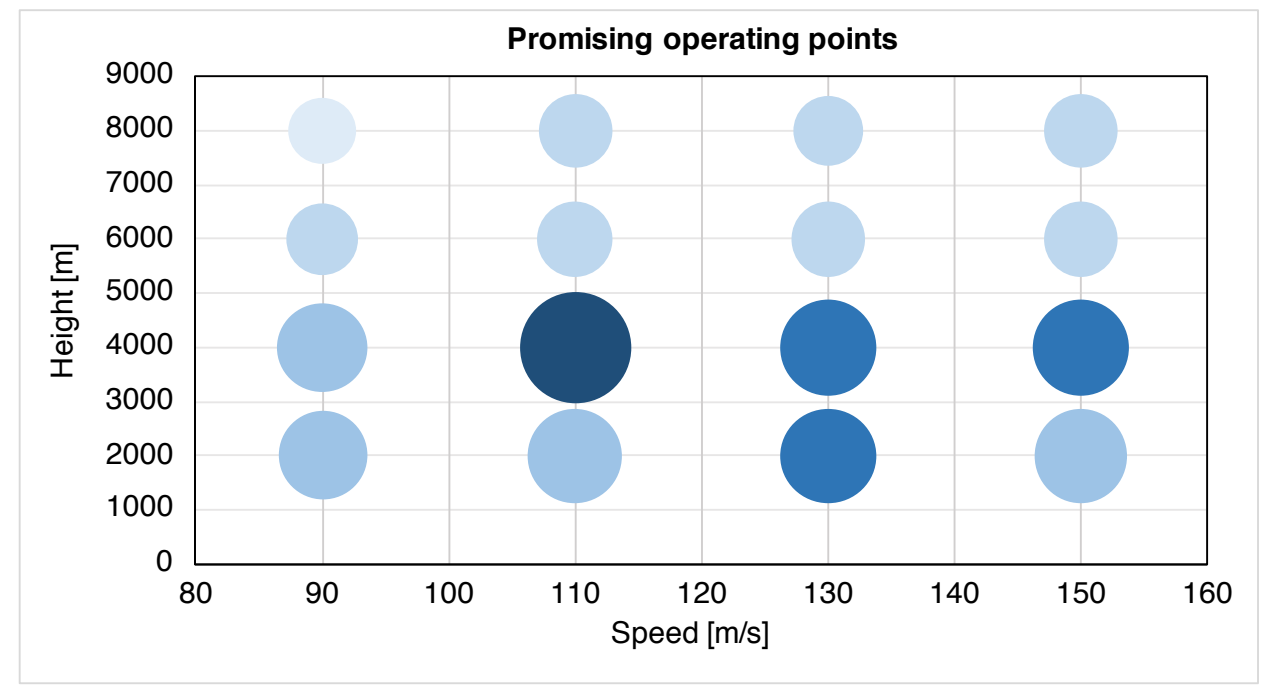

Figure 4.12: Variable stability system limitations in flight envelope

The most promising operating point can be found at $4000 \mathrm{~m}$ altitude and a speed of $110 \mathrm{~m} / \mathrm{s}$. The maximum commanded pitch acceleration at this point is around $8 \mathrm{deg} / \mathrm{s}^{2}$. All exact values can be found in Appendix A. At this operating point, the initial deflection of the elevator to achieve straight and steady flight is close to zero. This is beneficial, because this enables a higher deflection of the elevator since the hinge moment increases at a lower rate with increasing elevator deflection compared to other conditions. It can be concluded that the Cessna Citation will be able to follow the highest commanded pitch acceleration in this operating point, which means that the variable stability system has the highest potential at this flight condition. 



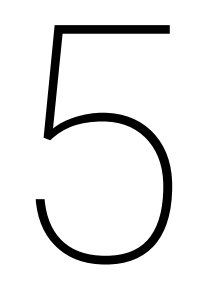

\section{Initial application}

Now that the initial limitations of the dynamic inversion variable stability systems are researched, the combined actuator and aircraft model can be used for manoeuvre simulations. The first thing that is investigated, is the rate of climb limitations due to the fly-by-wire system. Secondly, the software for the CAP determination is verified.

\subsection{Rate of climb check}

One of the initial problems in the experiments of Mirza et al. [3] was that pilots could not control the aircraft in normal manoeuvres. Pilots were asked to climb 1000 feet with a rate of climb between 500 and 1000 feet $/ \mathrm{min}$. However, due to the error described in Chapter 3, the elevator deflections were very small and the pilots could not perform their tasks. To investigate the capabilities of the newly validated model, a very basic rate of climb controller is designed and tested at the optimal operating condition found in the previous chapter (altitude of $4000 \mathrm{~m}$ and $110 \mathrm{~m} / \mathrm{s}$ true airspeed). The result of this can be found in Figure 5.1.
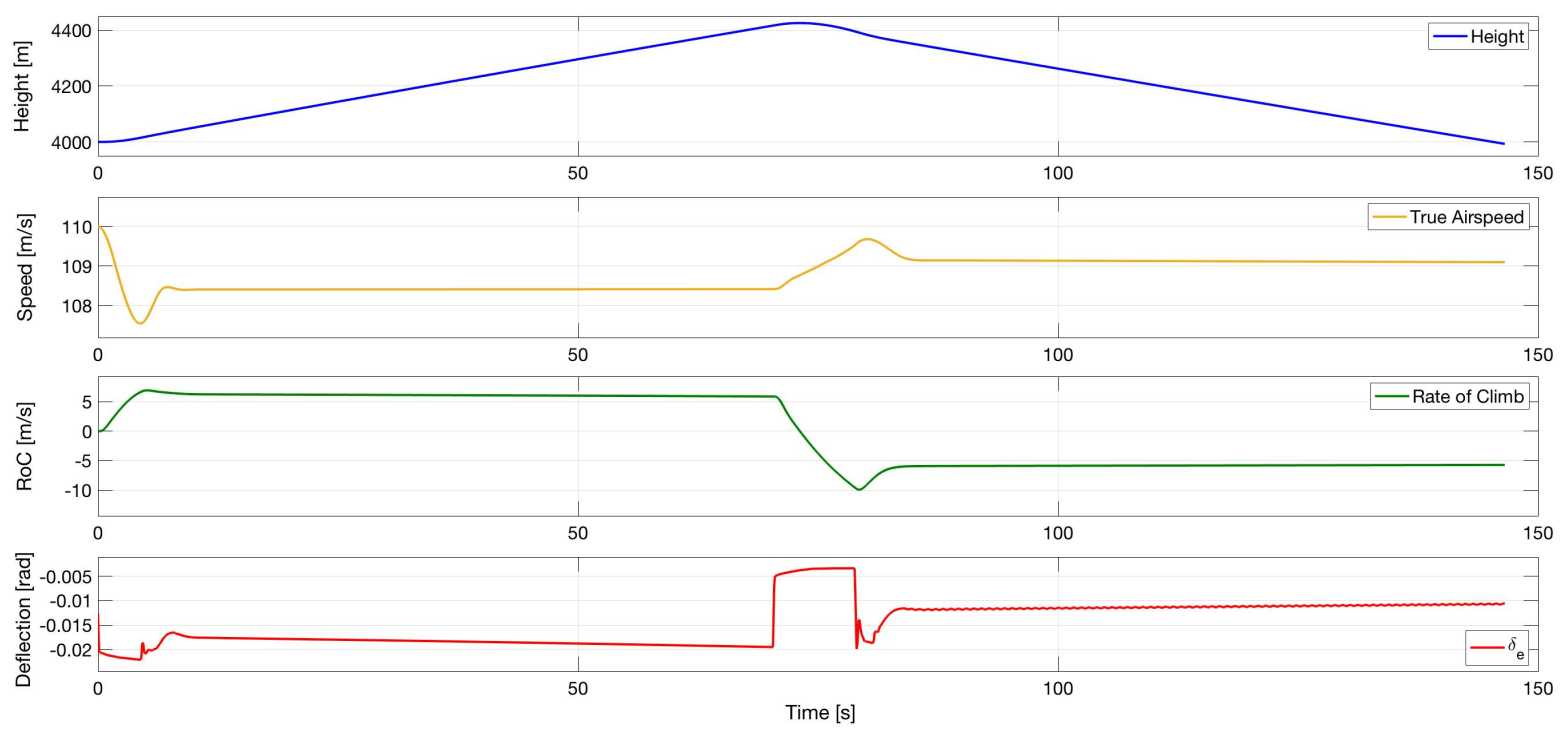

Figure 5.1: Controlled rate of climb $(+/-6 \mathrm{~m} / \mathrm{s})$ with combined actuator and aircraft model

The rate of climb that is commanded is initially $6 \mathrm{~m} / \mathrm{s}$ after which it is changed at $t=70 \mathrm{~s}$ to $-6 \mathrm{~m} / \mathrm{s}$. This is equivalent to a rate of climb of about $+/-1200$ feet/min. As can be seen, the aircraft can follow the commanded rate of climb quite well. The elevator deflection is not saturated in this case, which would have been the case when the uncorrected actuator model would have been used. 
Another situation is depicted in Figure 5.2. In this case it is tried to find the limits in the system with regards to the maximum rate of climb that can be followed. A rate of climb of $12 \mathrm{~m} / \mathrm{s}$ (approximately $2400 \mathrm{feet} / \mathrm{min}$ ) is commanded, which can initially be followed. However, the actuator saturation is reached after about 15 seconds. From this point onward, the rate of climb cannot be followed correctly anymore. The same goes for the rate of climb of $-10 \mathrm{~m} / \mathrm{s}$, which can be followed initially until the saturation of the elevator is reached.
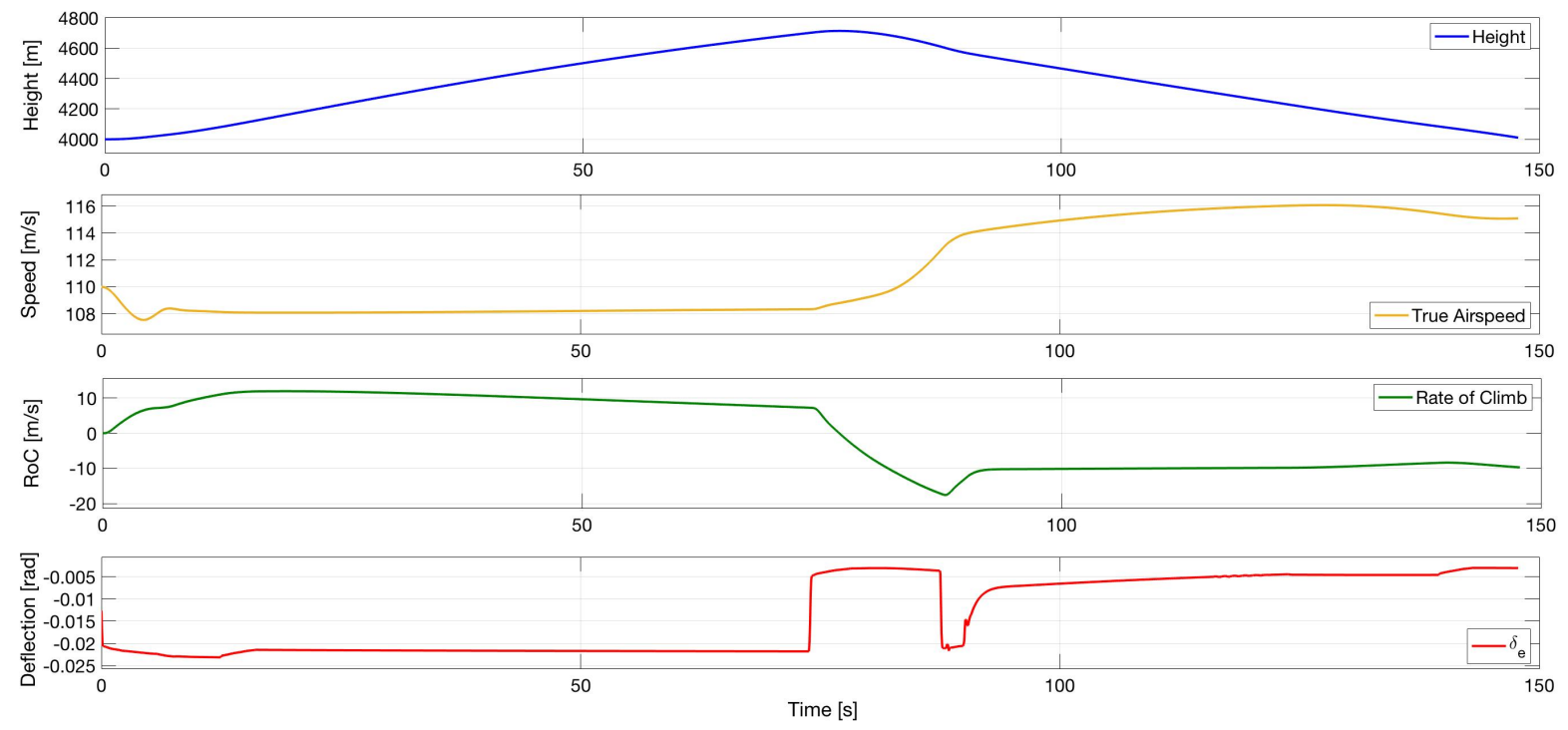

Figure 5.2: Controlled rate of climb $(+12$ and $-10 \mathrm{~m} / \mathrm{s})$ with combined actuator and aircraft model

This shows what kind of longitudinal manoeuvres can be performed when an experiment would be performed regarding the variable stability system. The rate of climb that can be sustained is limited at approximately 1600 feet/min in this operating point. If the validated actuator model would be used in the experiment by Mirza, the pilots would have been able to fly the requested experiment.

The maximum rate of climb for all other initial conditions is presented in Table 5.1. It should be noted that this rate of climb cannot be sustained because of the elevator deflection limitation. If a manoeuvre has to be selected in one of these flight conditions, it is suggested to subtract $35 \%$ of this maximum rate of climb. After this reduction, the rate of climb can be sustained without reaching the actual limitations of the actuator.

Table 5.1: Maximum rate of climb due to elevator deflection limitations

\begin{tabular}{llllll}
\hline \hline $\mathbf{H}[\boldsymbol{m}]$ & $\mathbf{V}[\mathbf{m} / \mathbf{s}]$ & Max RoC $[\boldsymbol{m} / \boldsymbol{s}]$ & Max RoC $[\mathbf{f e e t} / \mathbf{m i n}]$ & Min RoC $[\boldsymbol{m} / \mathbf{s}]$ & Min RoC $[\mathbf{f e e t} / \mathbf{m i n}]$ \\
\hline \hline 2000 & 90 & 12,85 & 2529,5 & $-16,32$ & $-3212,6$ \\
2000 & 110 & 13,26 & 2610,2 & $-20,62$ & $-4059,1$ \\
2000 & 130 & 13,97 & 2750,0 & $-21,92$ & $-4315,0$ \\
2000 & 150 & 12,04 & 2370,1 & $-21,35$ & $-4202,8$ \\
\hline 4000 & 90 & 11,98 & 2358,3 & $-13,98$ & $-2752,0$ \\
4000 & 110 & 11,99 & 2360,2 & $-16,84$ & $-3315,0$ \\
4000 & 130 & 12,27 & 2415,4 & $-18,68$ & $-3677,2$ \\
4000 & 150 & 11,73 & 2309,1 & $-20,19$ & $-3974,4$ \\
\hline 6000 & 90 & 10,45 & 2057,1 & $-15,62$ & $-3074,8$ \\
6000 & 110 & 11,09 & 2183,1 & $-18,35$ & $-3612,2$ \\
6000 & 130 & 12,34 & 2429,1 & $-19,19$ & $-3777,6$ \\
6000 & 150 & 11,14 & 2192,9 & $-20,62$ & $-4059,1$ \\
\hline 8000 & 90 & 9,64 & 1897,6 & $-16,92$ & $-3330,7$ \\
8000 & 110 & 11,05 & 2175,2 & $-19,33$ & $-3805,1$ \\
8000 & 130 & 11,57 & 2277,6 & $-22,63$ & $-4454,7$ \\
8000 & 150 & 12,35 & 2431,1 & $-23,52$ & $-4629,9$ \\
\hline \hline
\end{tabular}




\subsection{CAP determination}

In Section 2.3 the method of determining the CAP is described. This method has been translated to a software tool to be able to determine the CAP of our systems. This will be of importance when the controllers are designed. At this point, it is required to compare the CAP from the model that is given as an input to the variable stability system with the CAP of the total system. If these CAP's are matching, one can conclude that the system is working properly. If they are not matching, the system is probably reaching the variable stability limits. To determine what kind of CAP can be simulated by the system, it is required to find these limits.

To translate the HOS to LOES, the Equation Error and the Output Error method are used. They give the estimation as given in Figure 5.3.

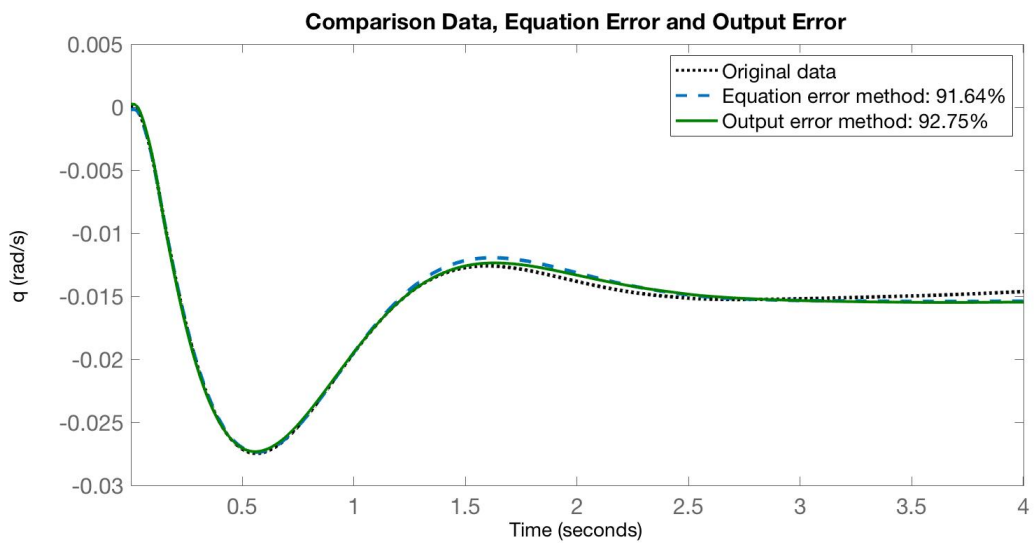

Figure 5.3: Equation Error and Output Error estimation

As expected, the Output Error method gives a better parameter estimation with the initial parameters of the Equation Error used as an input. From the estimated parameters in the LOES, the CAP can be determined. The CAP for the different initial conditions is given in Figure 5.4. It should be noted that these are determined without any of the variable stability control systems, and are thus the CAP's of the regular Cessna Citation 500. The actual values and damping coefficients for the initial conditions can be found in Appendix A.

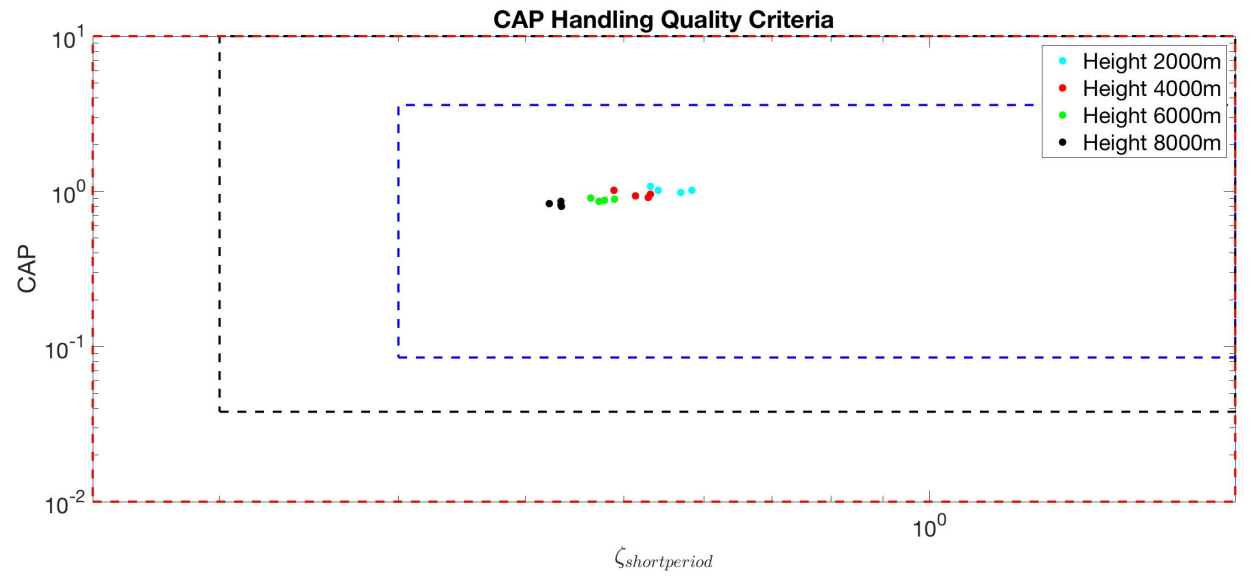

Figure 5.4: CAP estimation of the Cessna Citation 500 at different initial conditions 



\section{6}

\section{Conclusion and discussion}

Variable stability in-flight simulation has proven to be of great use when designing and testing new flight control logic. Next to this, it can be used for training pilots and testing of handling qualities. A variable stability system can reduce the cost of pilot training and could be used as a basis for other research at our faculty. This is why the section Control and Simulation decided to investigate this system in more detail, especially applied to their research aircraft the Cessna Citation II.

Three main different methods were identified to achieve a variable stability system, being feedback response, model-following and dynamic inversion respectively. The feedback response method has shown to be a simple method, which essentially functions as a proof of concept of variable stability. However, it requires gain scheduling for both the model to follow as the operating point at which this model is supposed to be followed. Next to this, literature shows that this technique is not able to follow all the reference states exactly, mainly because the host dynamics of the aircraft itself are not cancelled with this technique.

The second technique discussed is the model-following system. The implicit model-following, or also known as the model in the performance index system, has a similar structure compared to the feedback response method. However, now the gains are chosen in such a way that with this specific gain selection, a model can be followed exactly. This technique has been flown by CAL in the early seventies. A downside is that it again requires gain scheduling for both the model as the operating point. Next to this, it requires the derivative of the performance output, which is not always directly available. If a technique has to be introduced to differentiate within the control loop, this will introduce increased noise and time delays. Finally, this technique is only valid for a linearised system. Considering the aircraft and actuator model are both non-linear, this might introduce errors in the matching of the reference model.The other model-following technique discussed is the explicit model-following. This technique uses a model as a feedforward compensator. It has also been flown by CAL in the eighties and nineties. It requires gain scheduling for both the model to be followed as the operating point at which we are flying. It is also only valid for linearised systems.

The final technique discussed is the dynamic inversion. In all versions of dynamic inversion, the model to be followed is used as a feedforward reference input. All techniques are also able to cancel the host dynamics of the aircraft itself, which makes it more easy for the total system to follow the reference input. Linear dynamic inversion achieves this by using the linearised system of the aircraft inside the feedback loop. Non-linear dynamic inversion uses a full non-linear dynamic model inside the feedback loop. This is a downside, since the dynamic inversion is only as effective as the model that is used to inverse it. Next to this, it requires full state feedback and gain scheduling is still necessary to cancel the host dynamics of the aircraft at different operating points. A solution is given in the form of incremental non-linear dynamic inversion, where angular acceleration sensors are used in the feedback loop instead of a full aircraft model. Gain scheduling is now minimised only to the moment effectiveness parameter, which makes this a much more flexible solution. Also the uncertainties form the full model in the feedback loop are removed since this full model is not required anymore. This makes the system much more robust. Concluding, incremental non-linear dynamic inversion seems to be the most flexible system that cancels the host dynamics of the aircraft itself. 
The variable stability limits are investigated by finding the limits of the fly by wire system and the limits of the dynamic inversion method. Before the limits of the fly by wire system could be found, the actuator model had to be validated. Three issues in the actuator model were identified. The first one being the error in the hinge moment estimation, since the initial conditions could not be followed without any inputs. Secondly, the lack of deflection due to the control of the servo drums instead of the actuator deflection itself. Thirdly, the current limits of the servos could not be detected at the right timing, thus deflection of the actuators was possible after reaching the actual limiting currents. Having resolved these issues, the actuator model is validated and it could be used to determine the deflection limits of the fly by wire system. For the elevator, it was found that the maximum deflection would be at low speeds and high altitudes with a deflection of 1 degree.

The limits of the dynamic inversion are found to be related to the moment effectiveness parameter. This parameter was found to be highest at low altitudes and high speeds. When the maximum deflection and the moment effectiveness were combined, an optimal point in the flight envelope could be found with respect to the maximum pitch acceleration that can be followed by a variable stability system. For the Cessna Citation this was found to be at an altitude of $4000 \mathrm{~m}$ and a true airspeed of $110 \mathrm{~m} / \mathrm{s}$. The maximum pitch acceleration at this point is $8 \mathrm{deg} / \mathrm{s}^{2}$. This will be the maximum pitch acceleration that the Cessna Citation is able to follow without actual modifications to the aircraft itself.

In a previously conducted experiment by Mirza, the pilots were not able to control the aircraft to a specific rate of climb due to the errors in the actuator model. To check what type of manoeuvres the combined system can perform in such experiments, a rate of climb controller was designed to get an initial estimation. It was found that the rate of climb that can be sustained in the optimal operating point (altitude of $4000 \mathrm{~m}$ and 110 $\mathrm{m} / \mathrm{s}$ true airspeed) is limited by the maximum deflection of the elevator. This limitation occurs at around $1600 \mathrm{feet} / \mathrm{min}$, or $8 \mathrm{~m} / \mathrm{s}$. This is considered to be sufficient performance for these type of experiments. Next to this, a tool has been developed to determine the CAP of the combined aircraft and actuator model. This tool uses the Equation Error and Output Error method by Morelli to achieve a sufficient parameter estimation from the HOS to LOES.

\section{Future outlook}

The first next step would be to design some of the controllers that were found in literature and compare their performance. Whilst it was found that some controllers might be more flexible in implementation or with respect to gain scheduling, no specific data was found on performance differences. Theoretically it could be that some controllers achieve the same performance, but they might be more difficult to implement and use in practical situations. Next to this, the behaviour of controllers might differ when the saturation of the actuators is reached. Tho investigate this in more detail, the following controllers have been selected:

- Linear dynamic inversion controller

- Incremental-non linear dynamic inversion controller

These controllers are selected, since it gives the possibility to compare the performance impact of the linearisation. How does this influence the performance and is this influence significant when we stay close to the original operating point at which the system is linearised? Next to the performance impact, these controllers can also provide more insight in the way that saturation of the actuators is handled; does the linear controller provide less robust behaviour or is this still sufficient?

The impact of the actuator saturation to the variable stability and CAP should also be investigated. One could design the controllers in such a way that the actuators will never be saturated with the knowledge acquired in this preliminary research, or it can be accepted that they saturate sometimes and that this might influence the handling qualities. A model of the pilot can be introduced in offline simulation to investigate the impact of the pilot gain on the saturation of the system.

When these controllers have been developed, the CAP of the model that has been given as an input can be compared to the CAP of the total system. If these match, it can be concluded that the system is working properly and the model-following variable stability is achieved. The CAP limitations of the total system can be investigated and one can say which handling qualities can be modelled and which ones would be beyond the 
physical capabilities of the aircraft.

Finally some kind of experiment has to be designed to verify and validate the different controllers. This can be done in two ways. Either analytically, by checking the output of the aircraft with the expected output of the model, or more subjectively by having pilots determine the handling qualities of the aircraft using the Cooper-Harper rating scale, comparable to previous research. Preferably both methods are used to provide the most solid research results.

This preliminary thesis has researched the different variable stability methods, some of the applications and has shown the potential of the technology. The main thesis will focus on controller design, handling quality limitations and verification and validation of the controller. 



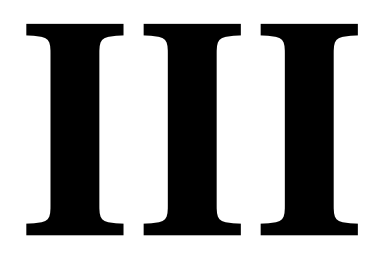

Appendices 



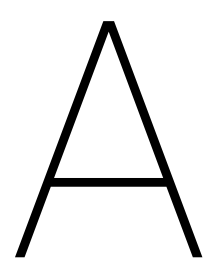

\section{Table of variable stability limitations}

In Table A.1 the different limiting values for the variable stability system can be found. Next to this, an initial CAP estimation is given for the different initial conditions. Note that this CAP is calculated without any control systems included, so it is just the CAP of the Cessna Citation 500 model itself. Note that this appendix is part of the preliminary thesis report, a description of the determination of these values can be found in that report.

Table A.1: Variable stability limitations for different initial flight conditions

\begin{tabular}{lllllllll}
\hline \hline $\mathbf{H}[\mathrm{m}]$ & $\mathbf{V}[\mathrm{m} / \mathrm{s}]$ & $\mathbf{C A P}[-]$ & Damp [-] & $\frac{M_{\delta_{e}}}{I_{y}}$ & $\frac{M_{\delta_{e}}}{I_{y}}[\%]$ & $\Delta \delta_{e}[\mathbf{r a d}]$ & $\dot{\Delta q}[\mathrm{rad} / \mathrm{s}]$ & $\dot{\Delta q}[\%]$ \\
\hline \hline 2000 & 90 & 1,0162 & 0,5833 & $-1,27 \mathrm{E}+01$ & $100,00 \%$ & 0,00881 & $1,12 \mathrm{E}-01$ & $100,00 \%$ \\
2000 & 110 & 0,9805 & 0,5692 & $-1,79 \mathrm{E}+01$ & $141,20 \%$ & 0,00667 & $1,19 \mathrm{E}-01$ & $106,90 \%$ \\
2000 & 130 & 1,0164 & 0,5403 & $-2,36 \mathrm{E}+01$ & $186,48 \%$ & 0,00507 & $1,20 \mathrm{E}-01$ & $107,31 \%$ \\
2000 & 150 & 1,0782 & 0,5315 & $-3,02 \mathrm{E}+01$ & $238,75 \%$ & 0,00390 & $1,18 \mathrm{E}-01$ & $105,69 \%$ \\
\hline 4000 & 90 & 0,9552 & 0,5312 & $-1,04 \mathrm{E}+01$ & $81,88 \%$ & 0,01080 & $1,12 \mathrm{E}-01$ & $100,38 \%$ \\
4000 & 110 & 0,9134 & 0,5284 & $-1,79 \mathrm{E}+01$ & $141,04 \%$ & 0,00786 & $1,40 \mathrm{E}-01$ & $125,81 \%$ \\
4000 & 130 & 0,9336 & 0,5134 & $-1,98 \mathrm{E}+01$ & $156,52 \%$ & 0,00603 & $1,20 \mathrm{E}-01$ & $107,16 \%$ \\
4000 & 150 & 1,0164 & 0,4888 & $-2,52 \mathrm{E}+01$ & $199,15 \%$ & 0,00474 & $1,20 \mathrm{E}-01$ & $107,15 \%$ \\
\hline 6000 & 90 & 0,8914 & 0,4898 & $-8,43 \mathrm{E}+00$ & $66,59 \%$ & 0,01070 & $9,02 \mathrm{E}-02$ & $80,88 \%$ \\
6000 & 110 & 0,8763 & 0,4787 & $-1,24 \mathrm{E}+01$ & $97,98 \%$ & 0,00763 & $9,46 \mathrm{E}-02$ & $84,85 \%$ \\
6000 & 130 & 0,8644 & 0,4729 & $-1,66 \mathrm{E}+01$ & $131,04 \%$ & 0,00568 & $9,42 \mathrm{E}-02$ & $84,46 \%$ \\
6000 & 150 & 0,9017 & 0,4637 & $-2,09 \mathrm{E}+01$ & $165,44 \%$ & 0,00452 & $9,46 \mathrm{E}-02$ & $84,80 \%$ \\
\hline 8000 & 90 & 0,8151 & 0,4334 & $-6,80 \mathrm{E}+00$ & $53,73 \%$ & 0,01242 & $8,45 \mathrm{E}-02$ & $75,75 \%$ \\
8000 & 110 & 0,8649 & 0,4335 & $-9,95 \mathrm{E}+00$ & $78,56 \%$ & 0,00938 & $9,33 \mathrm{E}-02$ & $83,67 \%$ \\
8000 & 130 & 0,8020 & 0,4342 & $-1,38 \mathrm{E}+01$ & $108,70 \%$ & 0,00638 & $8,79 \mathrm{E}-02$ & $78,77 \%$ \\
8000 & 150 & 0,8352 & 0,4225 & $-1,73 \mathrm{E}+01$ & $136,97 \%$ & 0,00544 & $9,43 \mathrm{E}-02$ & $84,53 \%$ \\
\hline \hline
\end{tabular}





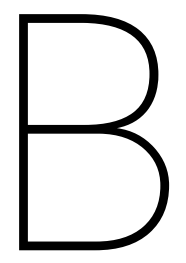

\section{Controller design}

This appendix describes the design of the controller that is used to achieve the variable stability in-flight simulator. The schematic block diagram of the controller structure is shown in Figure B.1. Note that this block diagram only shows the general outlines of the controller. The specifics regarding the implementation in MATLAB, Simulink and DUECA is discussed in Appendix C.

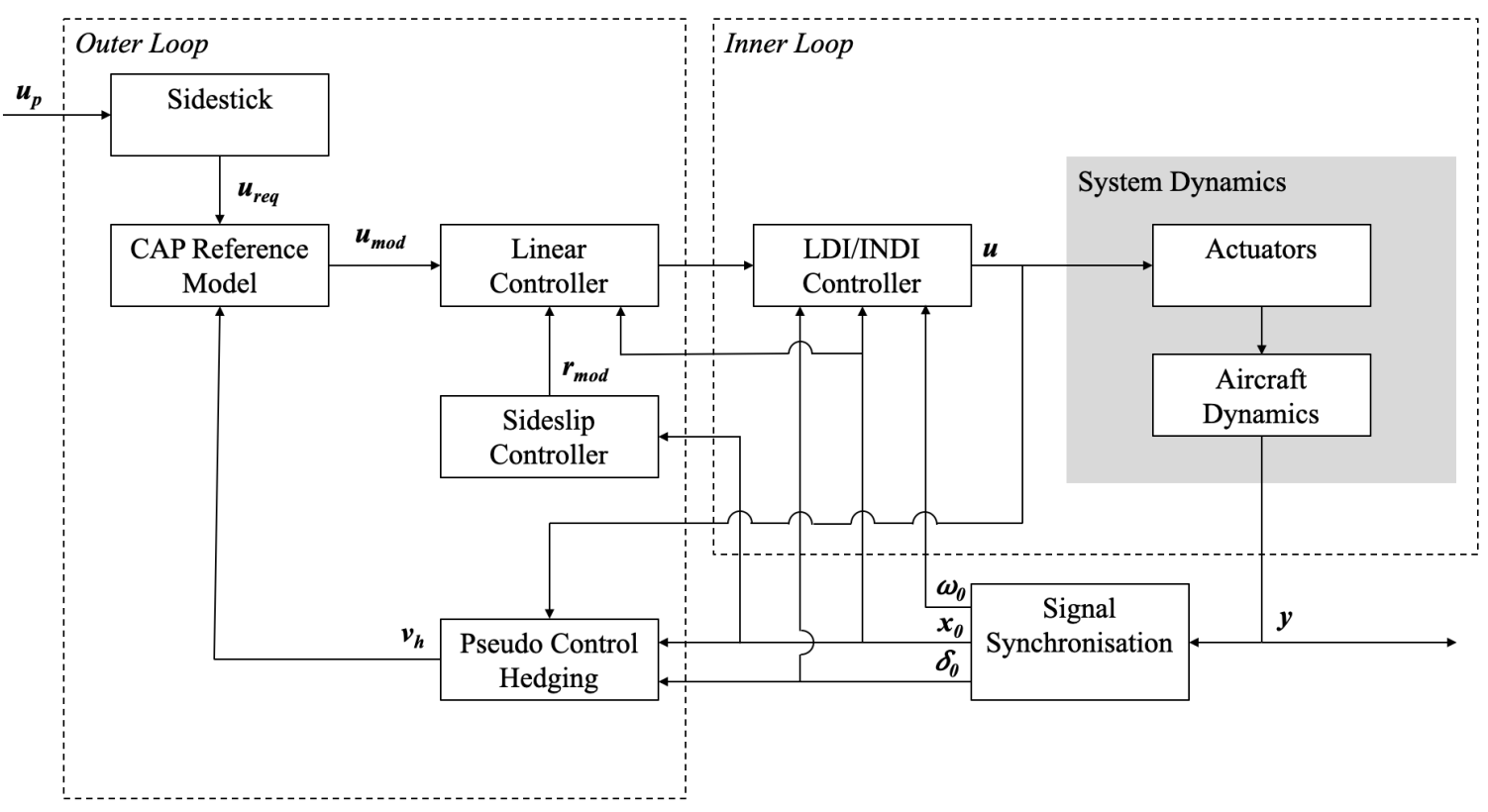

Figure B.1: Schematic block diagram of the controller structure

This appendix is structured as follows. Section B.1 describes the Linear Dynamic Inversion (LDI) and INDI logic. Next to this, a short description of the system dynamics is given. Secondly, section B.2 explains the CAP reference logic, the sideslip controller and the Pseudo-Control Hedging ( $\mathrm{PCH})$. Also the linear controller that minimises the error between the reference model and the aircraft states is introduced. 


\section{B.1. Inner loop design}

The inner loop consists of the system dynamics and the LDI and INDI control logic. The angular rates are controlled in both the LDI and INDI version of the controller. In contrast to previous work by Grondman et al. [36], the angular accelerations are now obtained from sensors inside a new Inertial Measurement Unit (IMU), instead of using a complementary filtering technique.

\section{B.1.1. LDI logic}

The LDI concept is derived in the preliminary work in subsection 2.2.3. The derivation amounts to the relation given in Equation B.1.

$$
u=(C B)^{-1}(\dot{r}+K e-C A x)
$$

In this equation, the error of the system to be controlled is given by $K e$. This control error is given as an input to the LDI logic, coming from the linear controller in the outer loop. The aircraft dynamics itself are linearised by using the moment effectiveness inside the feedback linearisation loop. Note that the moment effectiveness is only valid at a specific initial condition, which implies that the controller has to be gain scheduled for the different conditions that are flown. This leads to the implementation as given in Equation B.2, where $\boldsymbol{v}_{\boldsymbol{e}}=\left[\begin{array}{lll}e_{\phi} & e_{\theta} & e_{\psi}\end{array}\right]^{T}, \boldsymbol{\omega}_{\mathbf{0}}=\left[\begin{array}{lll}p & q & r\end{array}\right]^{T}, \boldsymbol{\delta}_{\mathbf{0}}=\left[\begin{array}{lll}\delta_{0_{a}} & \delta_{0_{e}} & \delta_{0_{r}}\end{array}\right]^{T}$ and $\boldsymbol{u}=\left[\begin{array}{lll}\delta_{a} & \delta_{e} & \delta_{r}\end{array}\right]^{T}$. The result of the LDI logic is depicted in Figure B.2.

$$
\boldsymbol{u}=\left[\begin{array}{ccc}
\frac{I_{x}}{M_{\delta_{a}}} & 0 & 0 \\
0 & \frac{I_{y}}{M_{\delta_{e}}} & 0 \\
0 & 0 & \frac{I_{z}}{M_{\delta_{r}}}
\end{array}\right]\left(\boldsymbol{v}_{\boldsymbol{e}}-\boldsymbol{\omega}_{\mathbf{0}}\right)+\boldsymbol{\delta}_{\mathbf{0}}
$$

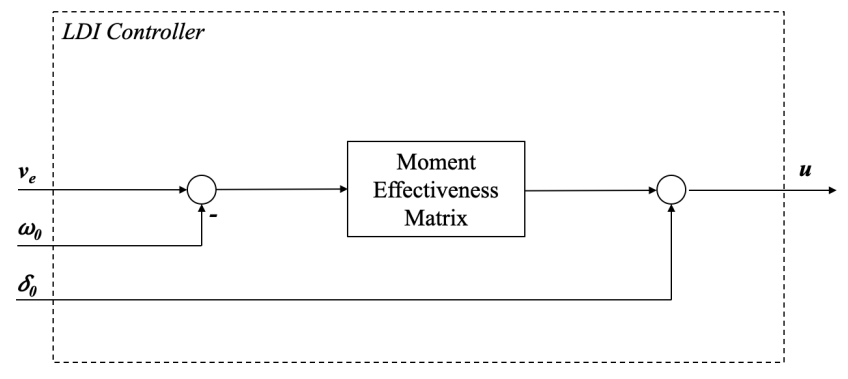

Figure B.2: Schematic block diagram of the LDI controller

\section{B.1.2. INDI logic}

The INDI controller is also introduced in the preliminary work in subsection 2.2.3, however, the mathematical derivation is not yet provided. The main difference between the LDI and INDI controller is that the INDI controller uses information from the aircraft model to adapt the moment effectiveness dependent on the flight condition. The aerodynamic moment $M_{A}$ can be split into three main components, being the moment induced by $M_{T}$, the moment induced by the control effectors $M_{c}$ and the airframe dependent moment $M_{a}$.

If the moment induced by the thrust is neglected, the airframe dependent moment and the moment induced by the control effectors can be described by Equation B.3 and Equation B.4 respectively. In these equations $V_{\text {tas }}$ is the true airspeed, $\rho$ is the density, $b$ is the span, $c$ is the mean aerodynamic chord, $S$ is the wing surface area and $C_{*}$ are the aircraft's dynamic coefficients. These dynamic coefficients are defined by the system dynamics and retrieved from the DASMAT model.

$$
\boldsymbol{M}_{\boldsymbol{a}}=\frac{1}{2} \rho V_{\text {tas }}^{2} S\left[\begin{array}{c}
b C_{l_{a}} \\
\bar{c} C_{m_{a}} \\
b C_{n_{a}}
\end{array}\right]
$$




$$
\boldsymbol{M}_{\boldsymbol{c}}=\frac{1}{2} \rho V_{t a s}^{2} S\left[\begin{array}{ccc}
b C_{l_{\delta_{a}}} & 0 & b C_{l_{\delta_{r}}} \\
0 & \bar{c} C_{m_{\delta_{e}}} & 0 \\
b C_{n_{\delta_{a}}} & 0 & b C_{n_{\delta r}}
\end{array}\right]
$$

Equation B.3-B.4 can be incorporated in the rotational dynamics of rigid-body aircraft over a flat non-rotating earth as given in the Newton-Euler equation, Equation B.5, where $J$ is the inertia tensor as given in Equation B.6. This leads to Equation B.7.

$$
\begin{gathered}
\dot{\boldsymbol{\omega}}=\boldsymbol{J}\left(\boldsymbol{M}_{\boldsymbol{A}}+\boldsymbol{M}_{\boldsymbol{T}}-\boldsymbol{\omega} \times \boldsymbol{J} \boldsymbol{\omega}\right) \\
\boldsymbol{J}=\left[\begin{array}{ccc}
I_{x} & 0 & -I_{x z} \\
0 & I_{y} & 0 \\
-I_{x z} & 0 & I_{z}
\end{array}\right] \\
\dot{\boldsymbol{\omega}}=\boldsymbol{J}\left(\boldsymbol{M}_{\boldsymbol{a}}+\boldsymbol{M}_{\boldsymbol{c}} \boldsymbol{u}-\boldsymbol{\omega} \times \boldsymbol{J} \boldsymbol{\omega}\right)
\end{gathered}
$$

The control law for INDI can be obtained by taking a first-order Taylor expansion around the current point in time, which is denoted by the subscript 0. This Taylor expansion can be found in Equation B.8.

$$
\begin{aligned}
\dot{\omega} \approx \dot{\omega}_{0}+\left.\frac{\partial}{\partial \omega}\left(J^{-1}\left(M_{a}+M_{c} u-\omega \times J \omega\right)\right)\right|_{\omega=\omega_{0} u=u_{0}}\left(\omega-\omega_{0}\right) & \\
& +\left.\frac{\partial}{\partial u}\left(J^{-1}\left(M_{a}+M_{c} u-\omega \times J \omega\right)\right)\right|_{\omega=\omega_{0} u=u_{0}}\left(u-u_{0}\right)
\end{aligned}
$$

Since the controller will be running at $1000 \mathrm{~Hz}$ to ensure stability in the actuator model from Lubbers [35], the time increments are very small. This fact, combined with instantaneous control effectors $\left(\boldsymbol{\omega}-\boldsymbol{\omega}_{\mathbf{0}} \ll \boldsymbol{u}-\boldsymbol{u}_{\mathbf{0}}\right)$ and assuming that $\boldsymbol{\omega}-\boldsymbol{\omega}_{\mathbf{0}}=0$, leads to a reduction of Equation B.8 to Equation B.9, where $\Delta \boldsymbol{u}=\boldsymbol{u}-\boldsymbol{u}_{0}$.

$$
\dot{\omega} \simeq \dot{\omega}_{0}+J^{-1} M_{c} \Delta u
$$

Inversion of Equation B.9 leads to the INDI control law as implemented in the controller. The result can be seen in Equation B.10, where $\dot{\boldsymbol{\omega}}$ has been replaced by the input from the linear controller $\boldsymbol{v}_{\boldsymbol{e}}$ and the current control surface deflections have been added. A schematic block diagram of this control law is depicted in Figure B.3.

$$
u=M_{c}^{-1} J\left(v_{e}-\dot{\omega_{0}}\right)+\delta_{0}
$$

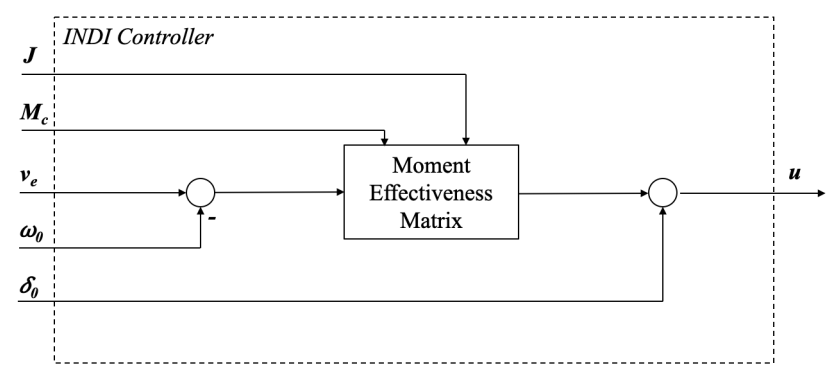

Figure B.3: Schematic block diagram of the INDI controller

\section{B.1.3. System dynamics}

The integration of the system dynamics is already described in the preliminary work of this thesis (see section 4.2), but will be recapped here shortly for completeness. The system dynamics are based on the DASMAT model of the Cessna Citation 500 described by Borst [33]. It has been modified to implement the non-linear actuator model from Lubbers [35]. The result of this implementation is shown in Figure B.4. 


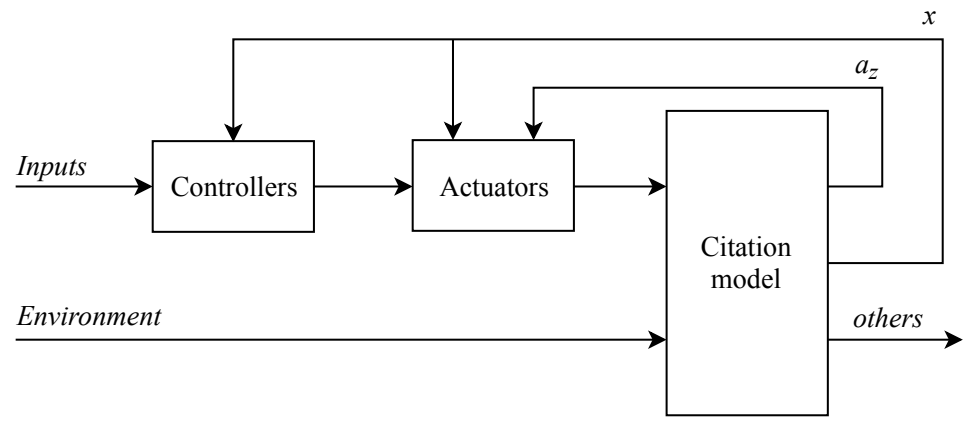

Figure B.4: Modified schematic overview of the CitAST model for the Cessna Citation 500

To be able to use both the non-linear actuator model and a more basic approximation of the actuators, a switch as been build inside the controller. The basic actuator model is based on a first-order approximation performed by Grondman et al. [36]. It has been updated to make use of the saturation flags from the full actuator model by Lubbers [35], to ensure the appropriate saturation deflections. The resulting logic is shown in Figure B.5, where gain 2 is the gain concerning the cable stretch and gain 1 represents the angular speed of the actuator. The schematic overview of the total actuator block can be found in Figure B.6.

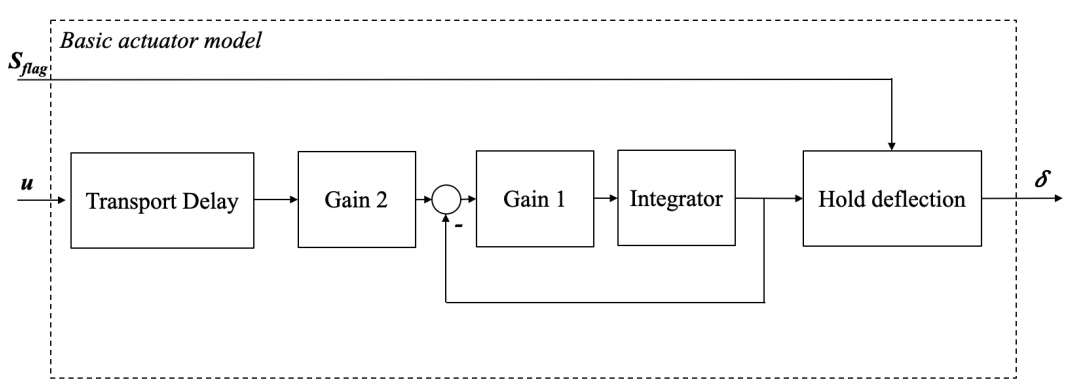

Figure B.5: Schematic overview of the modified basic actuator block

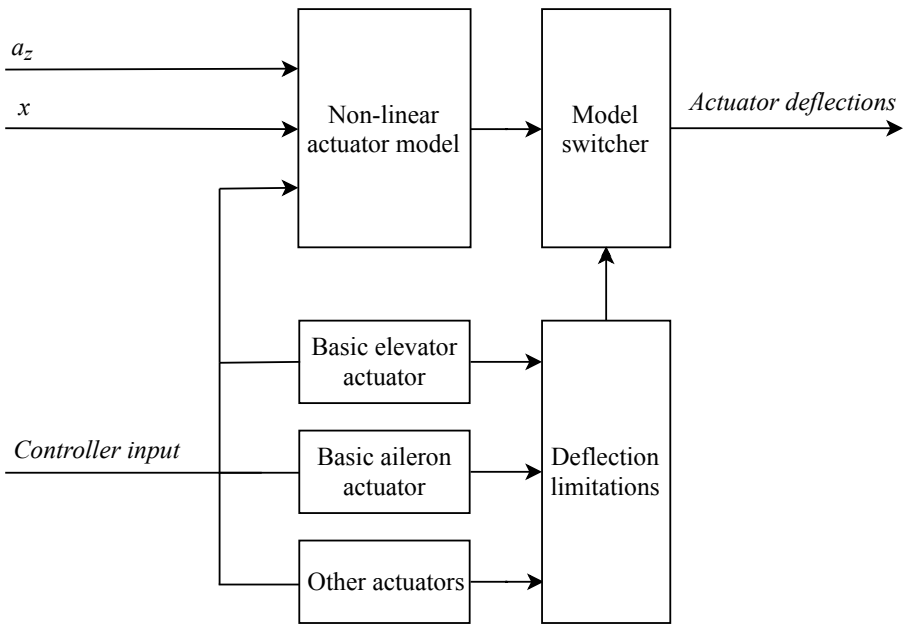

Figure B.6: Modified schematic overview of the Actuators block 


\section{B.2. Outer loop design}

The outer loop has been designed so it can be switched with other reference logic if required. Firstly the sideslip controller is discussed. Secondly the implementation of the PCH is investigated. Thirdly the CAP reference logic is explained and the side-stick gains are determined. Finally the linear controller is designed to ensure proper following of the CAP reference logic.

\section{B.2.1. Sideslip Controller}

The Cessna Citation II fly-by-wire hardware does not include a yaw input device, which means that coordinated flight can only be achieved when the sideslip is controlled. This has been recognised by Grondman et al. [36], who developed a PI controller to control the sideslip to zero. This is described in Equation B.11, where $K_{\beta}=1.93$ and $K_{\beta_{I}}=0.977$.

$$
r_{\text {mod }}=\frac{1}{V_{\text {tas }}}\left(V_{\text {tas }} \alpha p-f_{y}\right)\left(\frac{1}{s} K_{\beta_{I}}\left(\beta_{c m d}-\beta\right)-K_{\beta} \beta\right)
$$

\section{B.2.2. Pseudo-Control Hedging}

Johnson and Calise [37] developed a technique to compensate for actuator limitations by modifying the reference model dynamics. This technique is called PCH. Since actuator saturation will be an issue because of the limited fly-by-wire system performance, this technique has been implemented in the controller. $\mathrm{PCH}$ uses the inverse of the moment effectiveness matrix to correct for the differences between the commanded deflection by the INDI controller and the actual deflection of the actuators. This provides the hedging signal as given in Equation B.12.

$$
v_{h}=M_{c} J^{-1}\left(u-\delta_{0}\right)
$$

Looye et al. [38] activated the PCH only when saturation was occurring, whilst Grondman et al. [36] always kept the PCH active. In the latter case, the reference logic is also corrected for limited actuator speed instead of only the actuator position, which is why this method is preferred.

\section{B.2.3. CAP Reference Logic}

The CAP reference logic in pitching motion is designed to ensure a requested CAP and damping using the second order approximation as determined by Bischoff [28]. The linear controller uses $\boldsymbol{u}_{\boldsymbol{m} \boldsymbol{m o d}}$ as an input, which is essentially a reference pitch and roll rate. Yaw is not controlled to zero by the controller, thus it is not present in the CAP reference logic. To convert the requested input $u_{r e q}$ to these reference rates, an estimation of both the pitch gain $K_{\theta}$ and the longitudinal coefficient $T_{\theta_{2}}$ are required as given in Equation 2.64. To achieve this estimation, the method from Morelli [31] is used. Since the CAP and damping are design parameters, Equation 2.64 can be rewritten to provide the final CAP reference model Equation B.13. The corresponding coefficients are given in Equation B.14.

$$
\begin{gathered}
\frac{u_{m o d_{q}}}{u_{r e q_{q}}}=\frac{a_{1} s+a_{2}}{s^{2}+b_{1} s+b_{2}} \\
a_{1}=K_{\theta} \\
b_{1}=2 \zeta_{q} \sqrt{\mathrm{CAP} \cdot \frac{V}{g} \frac{1}{T_{\theta_{2}}}} \quad b_{2}=\frac{K_{\theta}}{T_{\theta_{2}}}
\end{gathered}
$$

In rolling motion different reference dynamics are used as given in Equation B.15, with the coefficients defined as in Equation B.16. Grondman et al. [36] performed a design optimisation for these coefficients, resulting in $\zeta_{p}=1$ and $\omega_{p}=1.35$.

$$
\begin{gathered}
\frac{u_{\bmod _{p}}}{u_{r e q_{p}}}=\frac{c_{1}}{s^{2}+d_{1} s+d_{2}} \\
c_{1}=\omega_{p}^{2} \quad d_{1}=2 \zeta_{p} \omega_{p} \quad d_{2}=\omega_{p}^{2}
\end{gathered}
$$




\section{B.2.4. Sidestick}

The inputs from the pilots have to be scaled before they can enter the controller. Since both roll and pitch have different reference logic, both axis need different gains. The initial values were determined using a pilot model, where the gain was changed until the crossover frequency $\omega_{c}$ was equal to $2 \mathrm{rad} / \mathrm{s}$. The gains could be manually adapted in the software during the different experiments. This lead to the following standard stick gains: Pitch $K_{q}=0.0125$ and roll $K_{p}=0.65$.

\section{B.2.5. Linear Controller}

The linear controller was designed to follow the reference model as closely as possible. For the pitch and roll channel, the error for the state and the first derivative are controlled. Next to this, a feedforward gain was added to the second derivative. The result can be seen in Equation B.17-B.18.

$$
\begin{gathered}
v_{\dot{\phi}}=\left(K_{\phi}+\frac{K_{\phi_{I}}}{s}\right)\left(u_{\text {mod }_{p}}-\phi\right)+K_{\dot{\phi}}\left(\dot{u}_{\text {mod }_{p}}-\dot{\phi}\right)+K_{\ddot{\phi}} \ddot{u}_{\text {mod }_{p}} \\
v_{\dot{\theta}}=\left(K_{\theta}+\frac{K_{\theta_{I}}}{s}\right)\left(u_{\text {mod }_{q}}-\theta\right)+K_{\dot{\theta}}\left(\dot{u}_{\text {mod }_{q}}-\dot{\theta}\right)+K_{\ddot{\theta}} \ddot{u}_{\text {mod }_{q}}
\end{gathered}
$$

The sideslip was controlled in the yaw channel, without any pilot input. This controller can be seen in Equation B.19.

$$
\nu_{r}=K_{r}\left(r_{\text {mod }}-r\right)
$$

Grondman et al. [36] performed a robust parameter synthesis to optimise these gains. However, their design requirements are different in longitudinal motion, since they did not require exact model-following. A linear parameter grid search was performed to find the combination of gains that enabled better model-following performance. This resulted in two changes, where $K_{\dot{\theta}}$ was increased from 3.02 to 4.80 and $K_{\ddot{\theta}}$ increased from 0.524 to 0.70 . The final gains can be found in Table B.1.

Table B.1: Linear controller gains

\begin{tabular}{llll}
\hline \hline Roll & & Pitch & \\
$K_{\phi}$ & 5.51 & $K_{\theta}$ & 7.76 \\
$K_{\phi_{I}}$ & 1.34 & $K_{\theta_{I}}$ & 0.50 \\
$K_{\dot{\phi}}$ & 4.80 & $K_{\dot{\theta}}$ & 4.80 \\
$K_{\ddot{\phi}}$ & 1.05 & $K_{\ddot{\theta}}$ & 0.70 \\
\hline Yaw & & & \\
$K_{r}$ & 1.62 & & \\
\hline \hline
\end{tabular}




\section{C}

\section{Software}

The controllers have been developed and tested in offline simulations using MATLAB and Simulink. Section C.1 describes how these simulations can be run. Since multiple experiments had to be performed on different platforms, DUECA by van Paassen et al. [39] was required as a basic software platform. DUECA has been developed to act as a middle layer between hardware (simulators/research aircraft) and the actual control software itself. It is able to synchronise data from different sources and it can combine processes that are running at different frequencies. Different modules can be selected within the software, which makes the implementation on different platforms easier. The implementation in DUECA is described in section C.2.

\section{C.1. MATLAB/Simulink}

It was decided to create the controller in Simulink, since MATLAB is able to convert these models to C++. The code generated from MATLAB could be implemented within the DUECA software to enable rapid development and testing of the different controllers. The following steps have to be followed before a simulation can be run in the Simulink environment.

- Open and run initcit.m. This loads a specific trim file generated previously by the modified DASMAT trim routine as described in the preliminary thesis. All the initial conditions are loaded correctly within the MATLAB workspace to ensure Simulink can use these inital conditions correctly. The trimdatafile can be changed within the initcit.m script. If another trimfile is selected, this also has to be modified in the Simulink controller as described in the DASMAT documentation. It is however recommended to use the trimfile as selected, since this enables the best performance as described in the preliminary thesis.

- Open and run CAPplaceModel.m. This script calculates the gains required within the CAP reference model. These gains are saved in the MATLAB workspace so the controller can access them in Simulink. The CAP and damping can be changed within this script. If the trimfile has been changed from the original in the previous step, the computeCAP.m script has to be run again. This script calculates $T_{\theta_{2}}$ and the pitch gain $K_{\theta}$ for a specific initial condition.

- Now CSCitation.slx can be opened. This model includes all the different controllers and logic described in this thesis. Within the Simulink model you can select which controller to use and if you want to use $\mathrm{PCH}$. A pilot model is present that can be used for specific simulations. Many scopes are present to investigate the results from the offline simulation. Everything in the outer loop can be changed to generate different reference dynamics if required. 


\section{C.2. DUECA software}

\section{C.2.1. Platforms}

Three different platforms exist of the control software in the DUECA environment. Every platform is a different combination of modules, startups scripts and hardware. The first platform, called solo, was created to test the controller in an offline environment. This version has two different configurations, one where the inputs are given via an external stick and one where the inputs are given using a Graphical User Interface (GUI). These configurations can be selected in the dueca_mod.py files in the DUECA project.

The second version that was created is the one for the fixed-base simulation. This is the version runs the same software as in the solo environment, but the tasks are now distributed over multiple computers in the Human Machine Interaction (HMI) lab. For this version the inputs from the electrohydraulic stick are used. The outside visuals are generated using FlightGear, which were not present in the solo version.

The final version of the controller is the one that was flown in the aircraft. Multiple changes have been made to the controller with respect to the simulator version. These changes are listed below.

- The complete system dynamics were removed and changed to ensure that they would only output the moment effectiveness matrix. All other outputs were received from sensors based on the aircraft itself. The requested deflection that comes from the INDI controller was linked to the autopilot servos from the aircraft's fly-by-wire system.

- New angular accelerometers were installed on the Cessna Citation II. These were an addition to the previously available angular accelerations which were established by differentiating the angular rates. Second order filters were introduced to these angular accelerations to remove noise whilst still maintaining the original signal without too much time delay. Especially the differentiated angular rate signals consisted of a relatively high noise.

- The controller required a conversion of the stick gains, since the stick on the aircraft was not identical to the electrohydraulic stick in the fixed-base simulator. The stick on the aircraft converted the input from the pilots to a different scale, thus the gains were adapted to maintain the same scaling as in the simulator.

- The initial conditions that were used in the simulator experiment were switched with the actual conditions of the aircraft when the experimental control mode was selected. These initial conditions were continuously tuned when the experimental control mode was turned off. The updated initial conditions were also used in the determination of the moment effectiveness matrix.

- In the simulator version the DASMAT model was used, which consisted of an inertia tensor based on the Cessna Citation I. Since the experiment would be conducted in the Cessna Citation II, it was required to update this inertia tensor based on the newly developed model by Van den Hoek et al. [34]. The model mismatch between the DASMAT model and the new model was highest for the inertia around the y-axis $I_{y y}$, with a $35 \%$ mismatch. The $I_{x x}, I_{z z}$ and $I_{x z}$ showed a mismatch of $8 \%, 24 \%$ and $27 \%$ respectively.

\section{C.2.2. Schematic overview of the DUECA project}

A schematic overview of the DUECA project that was flown in the aircraft can be found on the next page. This is an overview generated by DUECA itself and it shows the different connections between software modules and the hardware. 


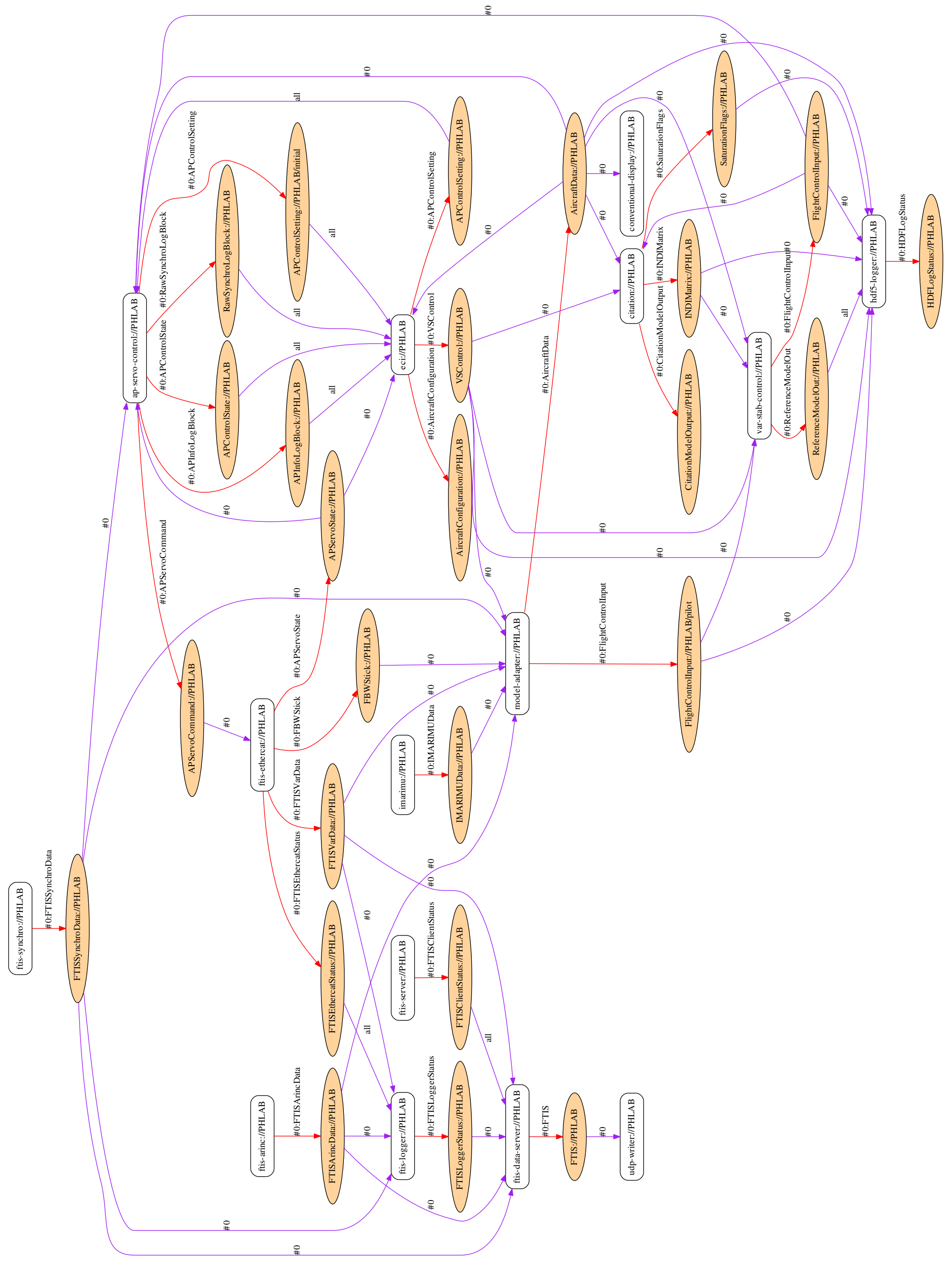




\section{C.2.3. How to control the software?}

Figure C.1 shows the variable stability software control screen. Different CAP and damping settings can be selected and loaded in the system. The experimenter has the choice to select either the IMAR IMU or the angular acceleration sensors. The control mode and actuator mode can be selected. In addition, the PCH can be activated or deactivated. Stick offsets and gains can be selected in both the $\mathrm{x}$ and $\mathrm{y}$ axis. A time delay can be introduced to the input of the pilot. The software starts logging when work is selected in the DUECA control panel, creating a log file name based on the time the log starts. Every time a setting changes in the variable stability control screen, this is logged as well.

The fly-by-wire system is controlled from the fly-by-wire system control screen as depicted in Figure C.2. The control tuning selects the gains used in the autopilot servo controller. Their standard value is $K_{p}=150$ with all other gains set to zero. In the channel selection and status screen, the experimenter can enable the different experimental control channels. If they are matched by the pilots in the cockpit, the experimental control mode can be initiated by the co-pilot.

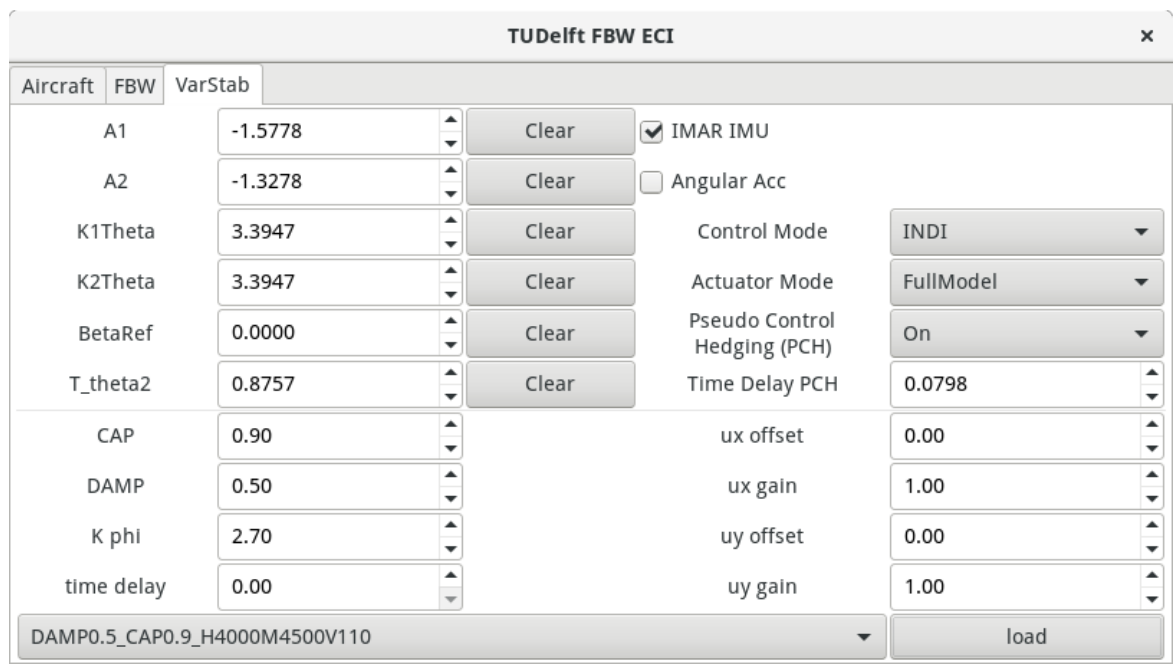

Figure C.1: Variable Stability Control Screen

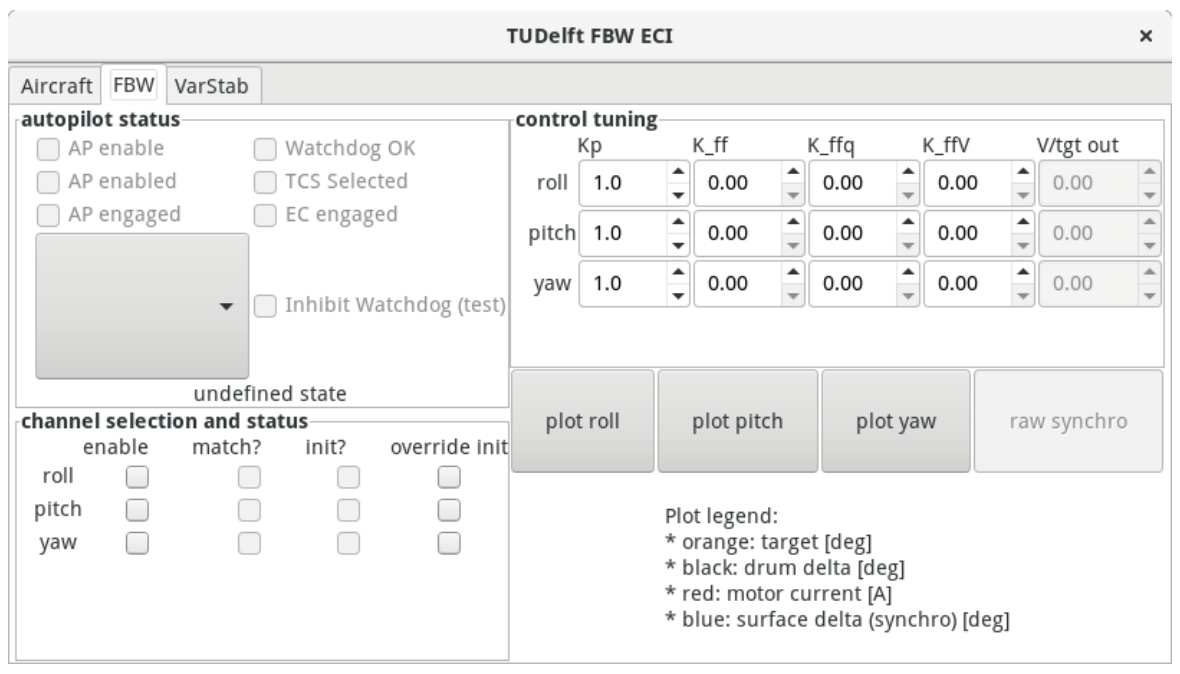

Figure C.2: Fly-by-wire system control screen 


\section{Offline simulations}

Before the final controller is chosen that is used during the experiments, some offline simulations in the Simulink model are performed to compare different aspects of the controller. A comparison is made between the LDI and INDI controllers. Two main factors are taken into account, being the performance of both controllers and the robustness to external influences. Next to this, the effect of the PCH is investigated. Finally, the response of the controllers to different CAP and damping inputs is simulated.

\section{D.1. Performance}

The performance differences between the controllers is investigated by comparing both controllers during pitch and roll manoeuvres. Both manoeuvres are performed at the initial condition of $4000 \mathrm{~m}$ altitude and a true airspeed of $110 \mathrm{~m} / \mathrm{s}$. This condition was found to be optimum with regards to moment effectiveness during the preliminary thesis work, taking the limitations of the fly-by-wire system into account. PCH is turned on for all tests.

\section{D.1.1. Pitch}

Figure D.1 shows the difference in response between both controllers when a step command is given on the pitch rate. The response variation that can be found in the figure shows the result of the same simulation, but with a maximum mismatch of $+/-50 \%$ in moment effectiveness between the model and the actual aircraft that is simulated. This mismatch is introduced to give an extra dimension to the comparison between the two controllers, since the simulated model will also have a mismatch compared to the actual aircraft. As can be seen in the figures, there is not a clear distinction in performance between the two controllers.

\section{D.1.2. Roll}

When looking at the response of the aircraft from a step input to the roll rate in Figure D.2, one can see a similar situation as for the pitch performance. No significant difference can be found between the two controllers. One point of interest that can be noticed is the response variation for the aileron deflection $\delta_{a}$ and the side slip $\beta$. This can be explained by the side slip controller that has to compensate in a different way when the model is not matching correctly to achieve the same performance. 

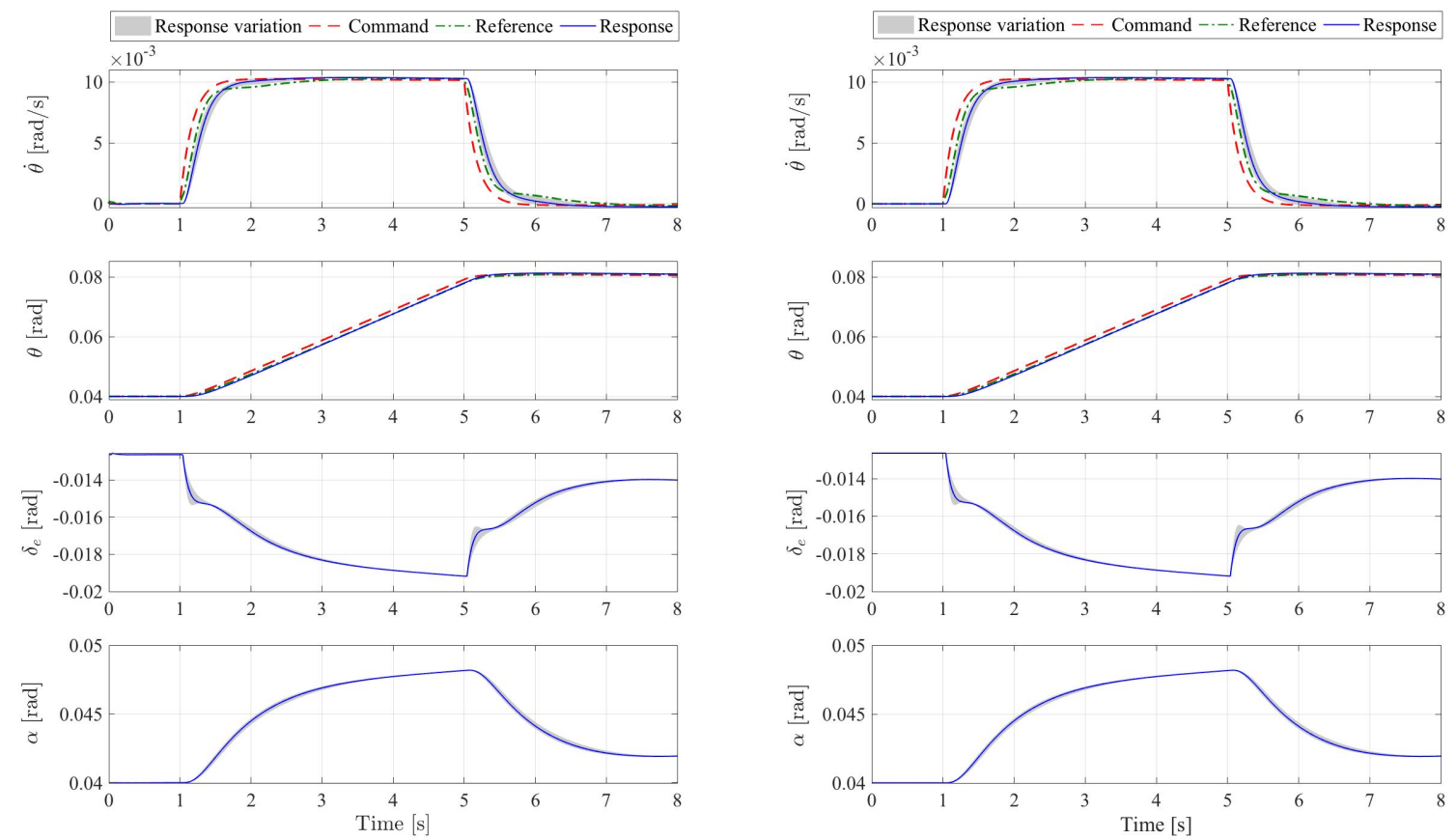

(a) LDI controller

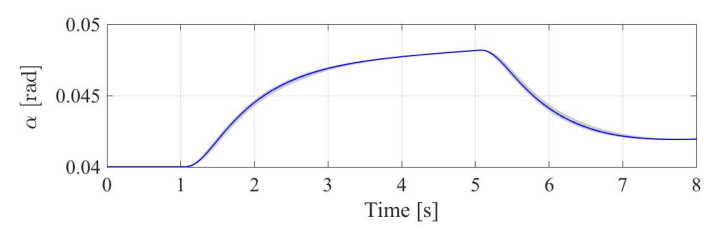

(b) INDI controller

Figure D.1: Aircraft pitch performance to step command on pitch rate
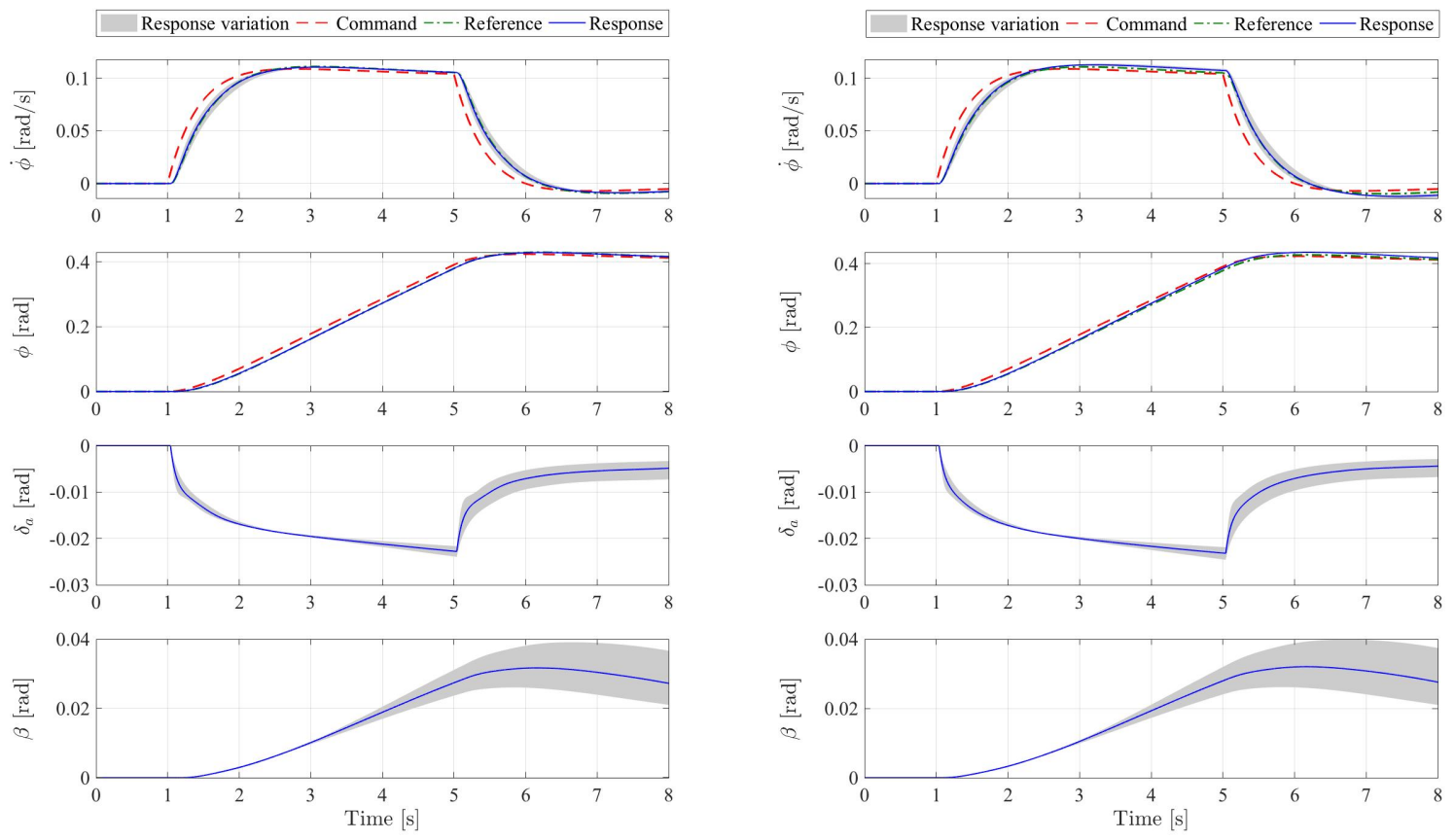

(a) LDI controller

(b) INDI controller

Figure D.2: Aircraft roll performance to step command on roll rate 


\section{D.2. Robustness}

The robustness differences between the controllers is also investigated by comparing both controllers during pitch and roll manoeuvres. Both manoeuvres are again performed at the initial condition of $4000 \mathrm{~m}$ altitude and a true airspeed of $110 \mathrm{~m} / \mathrm{s}$. PCH is turned on for all tests. In contrast to the performance simulations, the robustness simulations are based on the response of the controllers upon a $16 k t s$ wind disturbance. Different wind angles have been tested, but the scenario which caused the most significant difference between the controllers was found to be $+/$ - 45 degrees.

\section{D.2.1. Pitch}

Figure D.3 shows the difference in response in pitch when a $16 \mathrm{kts}$ wind disturbance at 45 degrees is applied after 1 second of simulation time. The differences between both controllers are minimal as can be seen from the Figure, but one should note the small difference in maximum pitch angle. The INDI controller handles this disturbance a little better compared to the LDI controller.

\section{D.2.2. Roll}

When looking at the roll motion, the difference between the controllers is more obvious. This can be seen in Figure D.4. Although the LDI controller reverts back to the commanded state quicker, it is a lot less robust when there is a model mismatch in the moment effectiveness. The INDI controller takes into account the coupling between sideslip and roll, which makes the correction of the controller more robust.

Based on the similar performance between the controllers and the higher robustness of the INDI controller, it was decided to continue to the experiments using the INDI controller only. 

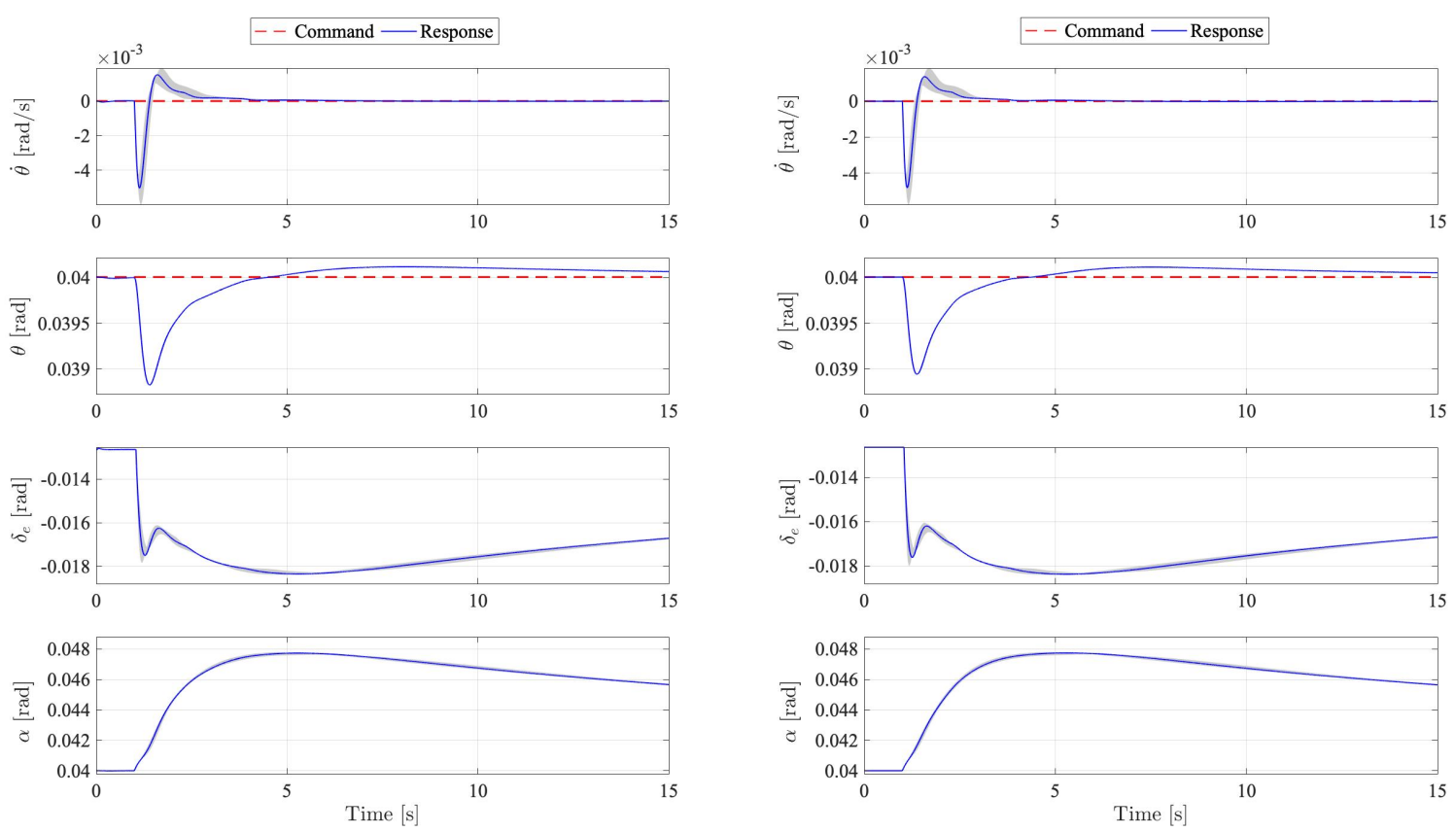

(a) LDI controller

(b) INDI controller

Figure D.3: Aircraft pitch response to 45 degrees 16 kts wind disturbance
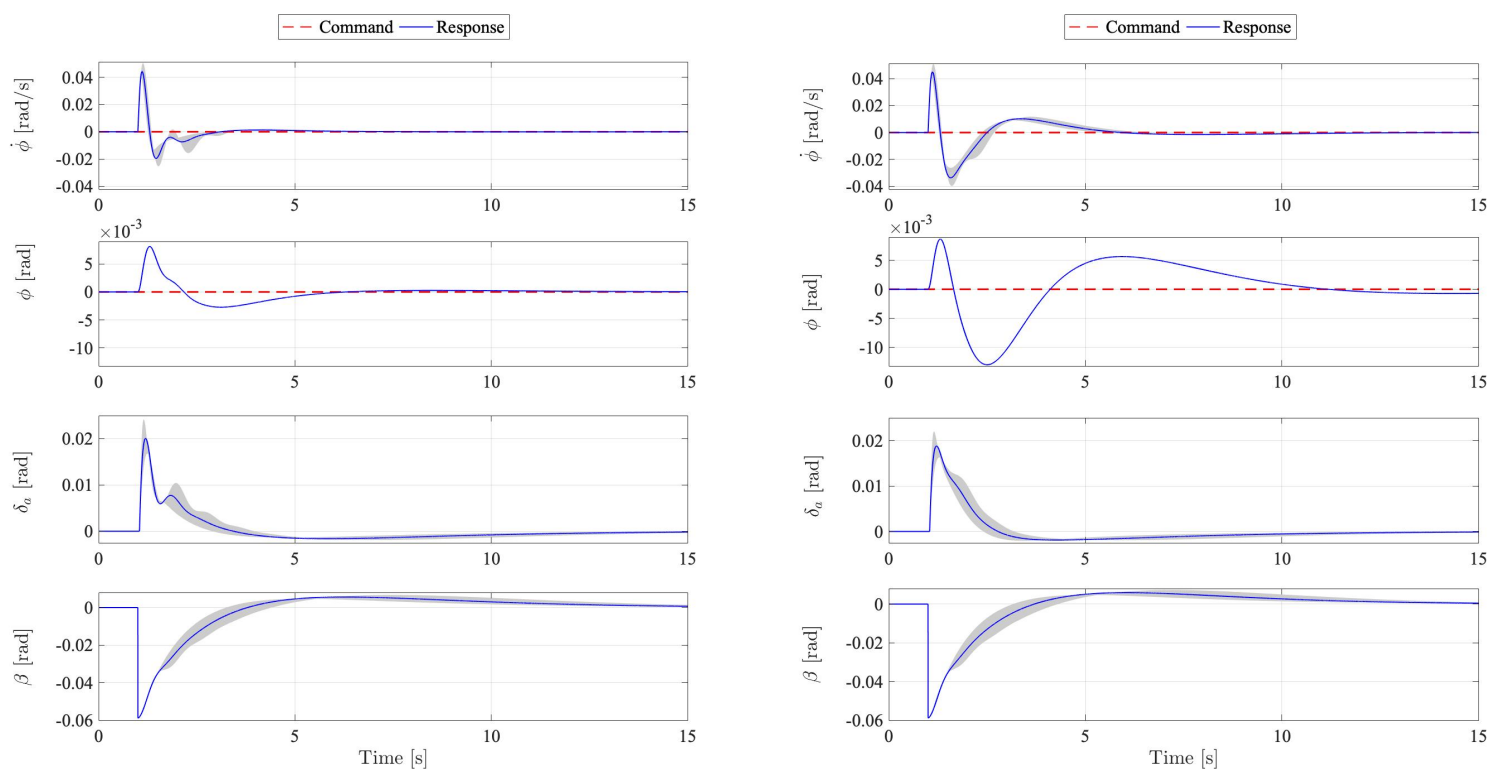

(a) LDI controller

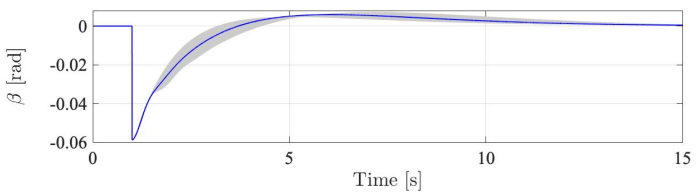

(b) INDI controller

Figure D.4: Aircraft roll response to 45 degrees 16 kts wind disturbance 


\section{D.3. CAP and Damping simulation}

This section shows the workings of the controller regarding the placement of the CAP and damping. Many different scenarios have been simulated, but two are chosen to show the working principle. In Figure D.5 the situations are depicted, one with a reference CAP of 0.6 and a reference damping of 1 , where the other simulation has a reference CAP of 1.1 and a reference damping of 0.3 . Both simulations are performed in the optimum point in the flight envelope as described before, with $\mathrm{PCH}$ turned on. Since the motion in pitch is the only motion of interest regarding the CAP, roll motion is neglected in this section.
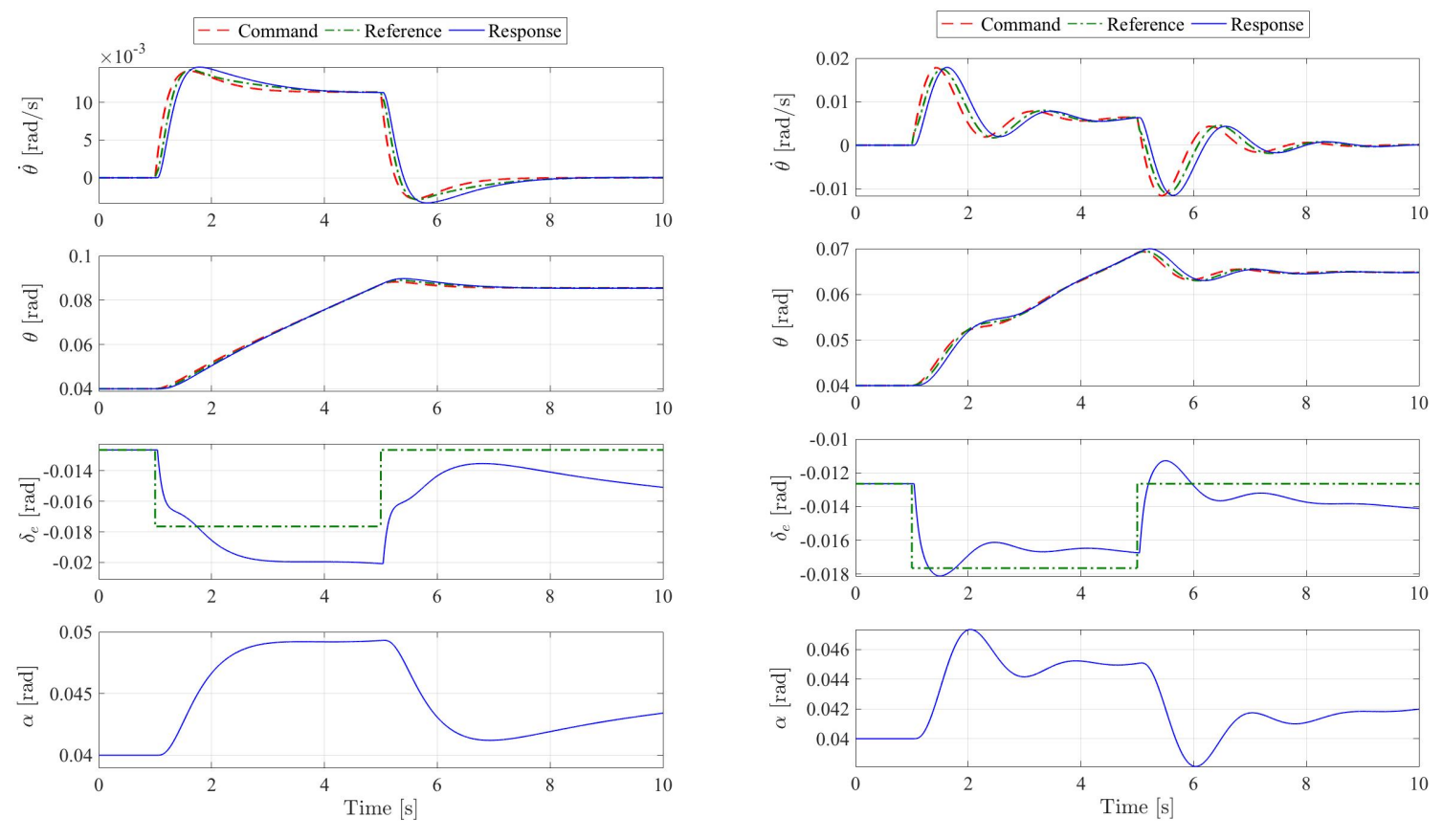

(a) $\mathrm{CAP}=0.6 ;$ Damping $=1.0$

(b) $\mathrm{CAP}=1.1 ;$ Damping $=0.3$

Figure D.5: Aircraft pitch response for different CAP and damping reference models, INDI controller with PCH on

The workings of the controller can be explained by looking at the deflection of the elevator. The reference deflection that enters the CAP and damping reference model is depicted by the green dotted line. One can see that this is for both situations the same input. However, to comply to the requested dynamics by the controller and the reference model, the actual elevator deflection is modified. This is depicted in the same Figure by the blue line. One can see that the elevator deflection is significantly different for both different reference CAP and damping, which causes the aircraft to respond according to the reference model.

A small delay occurs between the reference pitch rate and pitch angle, since a delay exists between the commanded elevator deflection and the actual elevator deflection. This delay is created due to physical limitations of the fly-by-wire system of the aircraft. Even though this delay exists, one can see that the requested response is still matched with high accuracy. 


\section{D.4. Pseudo Control Hedging}

$\mathrm{PCH}$ is used to improve the performance of the controller when the limits of the actuators are reached, both in actuator saturation and speed at which the actuator moves. The result of the PCH is displayed in Figure D.6.
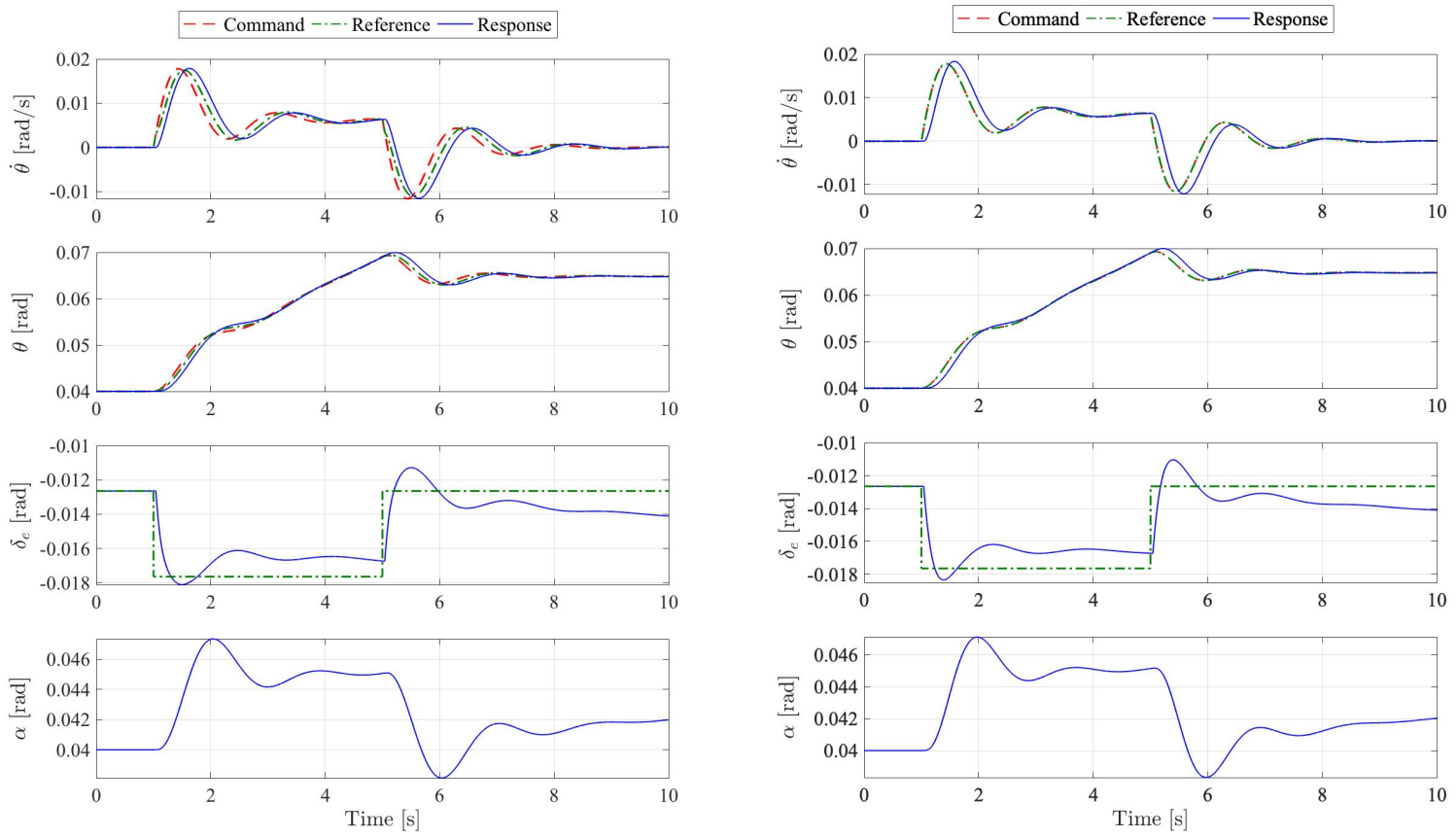

(a) $\mathrm{PCH}$ on

(b) PCH off

Figure D.6: Aircraft pitch response for different CAP 1.1 and damping 0.3 with the approximate actuator model

It can be seen that the amplitude of the pitch rate is matched a little better with the PCH turned on, compared to the controller without PCH. However, if the model of Lubbers [35] is used, some problems occur as can be seen in Figure D.7.

The model from Lubbers [35] appears to have a bigger delay compared to the approximate model designed from flight test data by Grondman et al. [36]. If the time delay term is not matched properly in the PCH controller, a mismatch occurs as can be seen in Figure D.7a. In this case the PCH controller acts as a damper compared to the controller where the $\mathrm{PCH}$ is turned off. This means that in this case the latter controller provides better CAP and damping matching capabilities. This is only a problem at lower reference damping rates, since the lower damping introduces more movement in the actuator to match this damping performance. However, since the approximate controller is designed from flight test data, it is considered to be a more reasonable choice when the concept has to be proven in the simulator. For this reason, the approximate model is used in the experiment. 

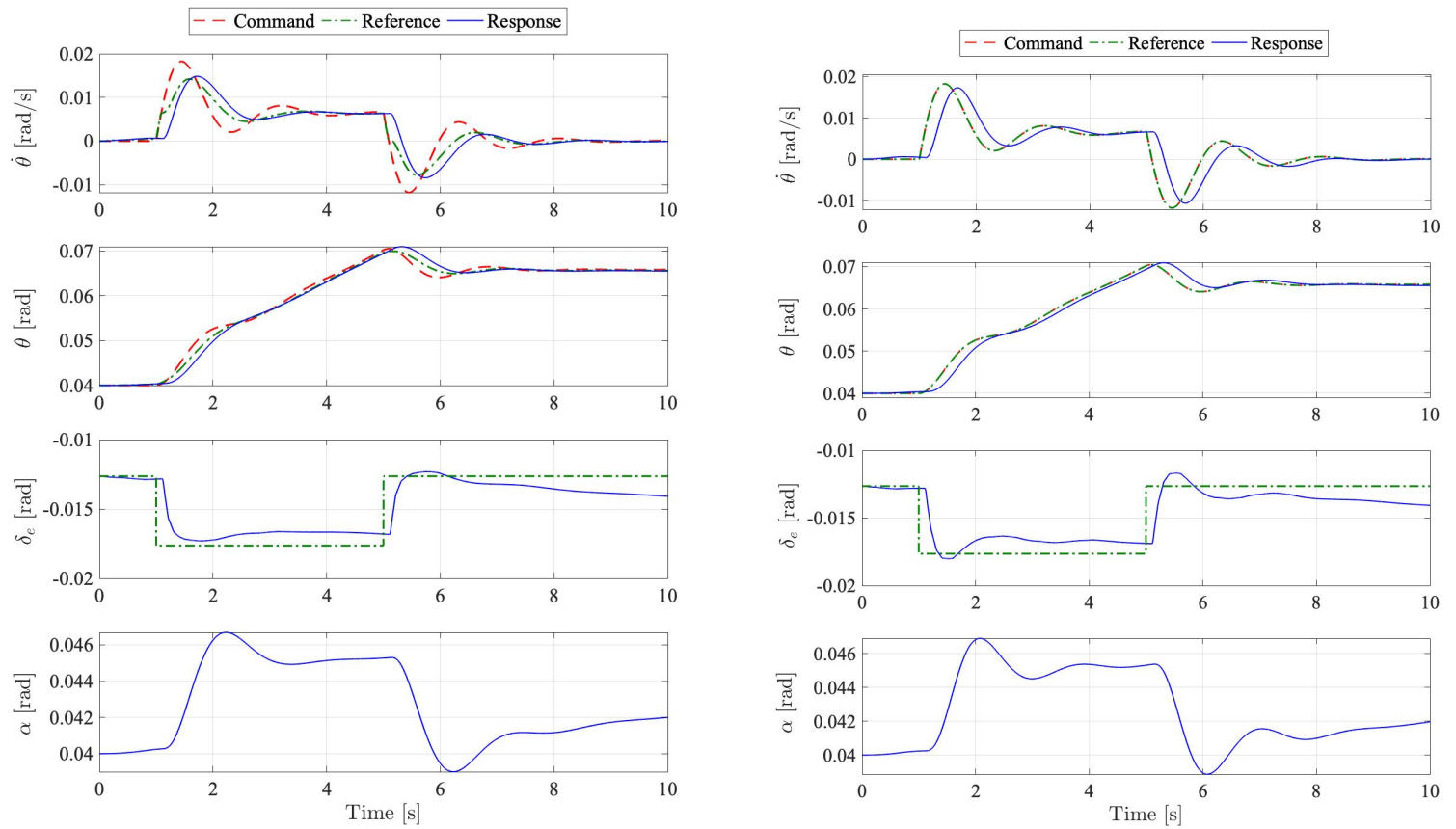

(a) PCH on

(b) PCH off

Figure D.7: Aircraft pitch response for different CAP 1.1 and damping 0.3 with the actuator model from Lubbers [35]

\section{D.5. CAP limits estimation}

The CAP limits could be estimated using the offline model. A pilot model was added to the controller which was setup to model different crossover frequencies. It was assumed that a pilot would feel an actuator saturation of 0.2 seconds, but this assumption can be updated with real data if the experiment in the fixed-base simulator is performed. Using this assumption, it can be found for which CAP and Damping settings saturation occurs for a longer sustained time than 0.2 seconds. The task that was given to the pilot model was to control the rate of climb to $\pm 1000 \mathrm{feet} / \mathrm{min}$. This task was chosen since it would be the same task that the pilots would have to perform in the fixed-base simulator experiment. The result of the expected lower limits can be seen in Figure D.8 for two different crossover frequencies.

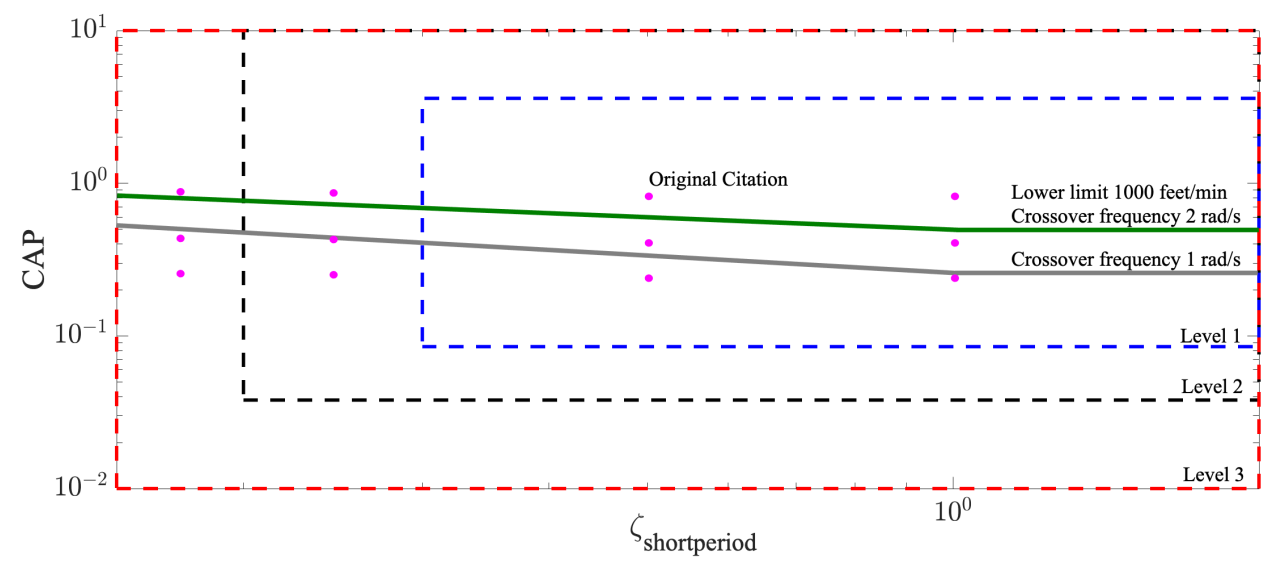

Figure D.8: Expected lower CAP limits due to actuator saturation 



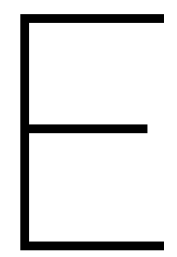

\section{Experiment I: Fixed-base simulator}

\section{E.1. Research Problem and Research Question}

A variable stability in-flight simulator is an aircraft which has the capabilities to change the stability coefficients in flight, often without changing the physical properties of the aircraft. It allows variations of response characteristics and handling qualities of an aircraft, which can be used for many different purposes like pilot training, control logic development and handling quality research. [1]

Control logic has been developed to make the Cessna Citation II aircraft from the faculty of Aerospace Engineering follow different types of responses regarding the Control Anticipation Parameter (CAP) and the Damping Ratio. The system uses the pilot input and converts it to a reference model for a specific CAP and damping ratio. The control logic tries to exactly follow this reference model to match its response. The limits of this logic are dependent on two different parameters, being the control task and the pilot gain (how the pilot responds to the aircraft). With this experiment it is investigated what the system limits are due to the actuator saturation for a specific control task. Next to this, it is researched if the pilots can feel the difference between the reference model and the complete dynamics of the reference model, controller and aircraft. This leads to the following research questions:

1. Can a pilot notice the differences between a CAP reference model and the full Incremental Nonlinear Dynamic Inversion model including aircraft dynamics?

2. What are the lower CAP limits of the system due to actuator saturation with a pilot in the loop experiment? 


\section{E.2. Experiment Plan}

In this chapter the experiment design is given. Firstly, the control variables are given. Secondly, the independent and dependent variables are described. From these variables the experiment matrix and amount of participants can be determined and finally a brief overview of the experimental procedures is presented.

\section{E.2.1. Control variables}

This section describes the control task, the controlled element and the displays/feedback.

\section{Control task}

The control task of the pilot will be the same during every test. The aircraft has to be controlled to a Rate of Climb of $1000 \mathrm{feet} / \mathrm{min}$ and $-1000 \mathrm{feet} / \mathrm{min}$. These conditions will be changed every ten seconds for a total of 10 changes for each run. This control task is chosen, since the many changes in Rate of Climb make it relatively easy for the pilot to notice the differences in CAP and Handling Qualities. The vertical speed or Rate of Climb is displayed on the Vertical Speed Indicator (VSI) as depicted in Figure E.1.

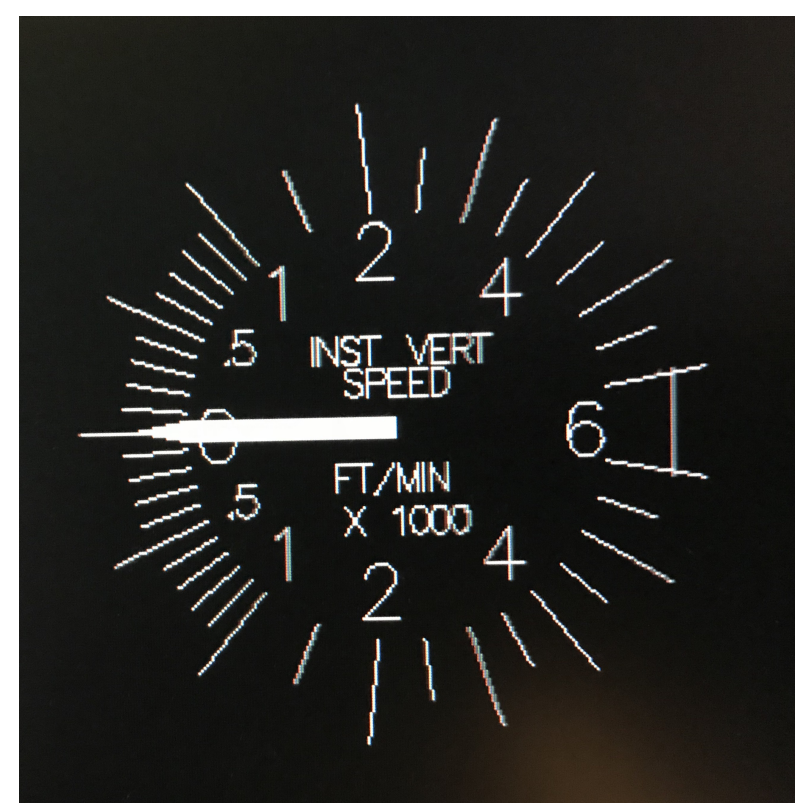

Figure E.1: Vertical Speed Indicator flight display

The initial condition that is chosen is at 4000 meters altitude and a true airspeed of $110 \mathrm{~m} / \mathrm{s}$. This initial condition is chosen since the preliminary research of this thesis has shown that within this condition, the best performance can be achieved when looking at both the moment effectiveness and the hinge moment in the elevator. It essentially means that the control task that can be performed in this initial condition can be more extreme (higher Rate of Climb) compared to other initial conditions.

\section{Controlled element}

For generating control inputs, a side stick is used. This side stick only controls the roll and pitch axes. The stick is a force-stick, which means that it physically does not deviate from its position, but it passes through the pilots input based on the amount of force that is exerted in a specific direction by the pilot. This is comparable to the force-stick that is present within the Cessna Citation II aircraft. 


\section{Displays and feedback}

The experiment will be held within the HMI lab at the faculty of Aerospace Engineering as can be seen in Figure E.2. Preferably, this experiment would have been executed in the SIMONA full motion research simulator, but sadly the SIMONA is in maintenance during the time of this writing.

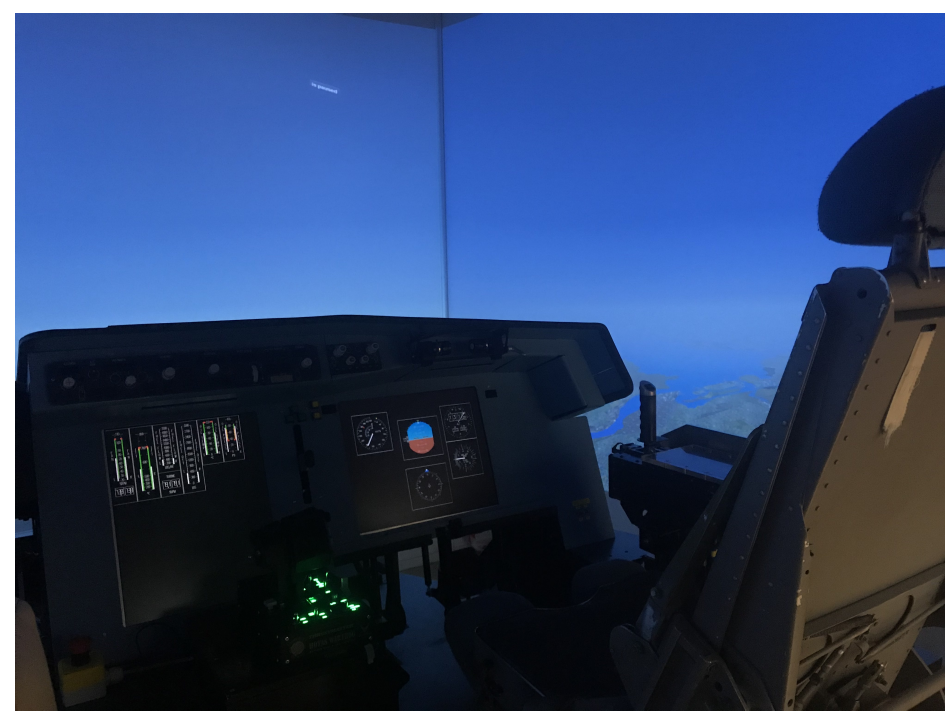

Figure E.2: HMI lab

To still ensure the pilot will achieve appropriate feedback of the different handling qualities, peripheral vision will be used in the experiment. The initial condition will be loaded above Schiphol Airport (AMS) in the Netherlands, with cloudy weather generated by the system. These clouds enhance the experience of the pilot in recognising the changes in handling qualities. Previous research has shown that pilots can notice the difference in CAP within the HMI lab in a similar test setup. Initial conditions have been chosen to ensure the pilot can notice the differences between them based on the results of this previous experiment.

The pilot will receive the information of the state of the aircraft using the primary flight display as given in Figure E.3. The pilot will mainly be focusing on the Vertical Speed Indicator as presented in the previous section, since the control task is based on this display. The information regarding the state of the engines and the fuel flow is depicted as shown in Figure E.4. These displays are chosen to mimic the real scenario in the Cessna Citation II as closely as possible.

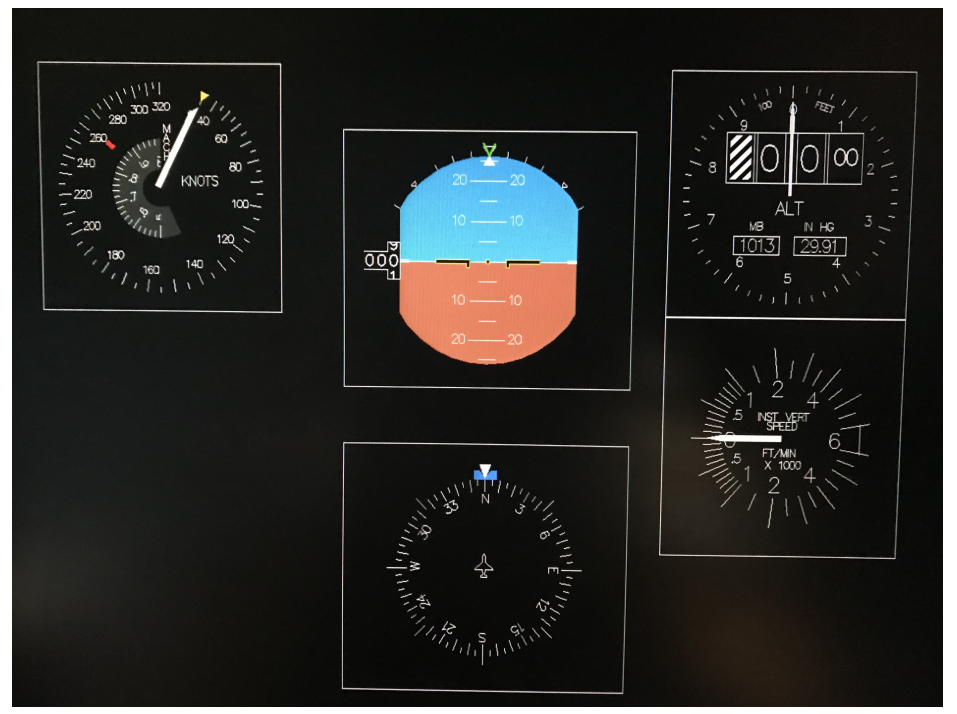

Figure E.3: Primary flight display 


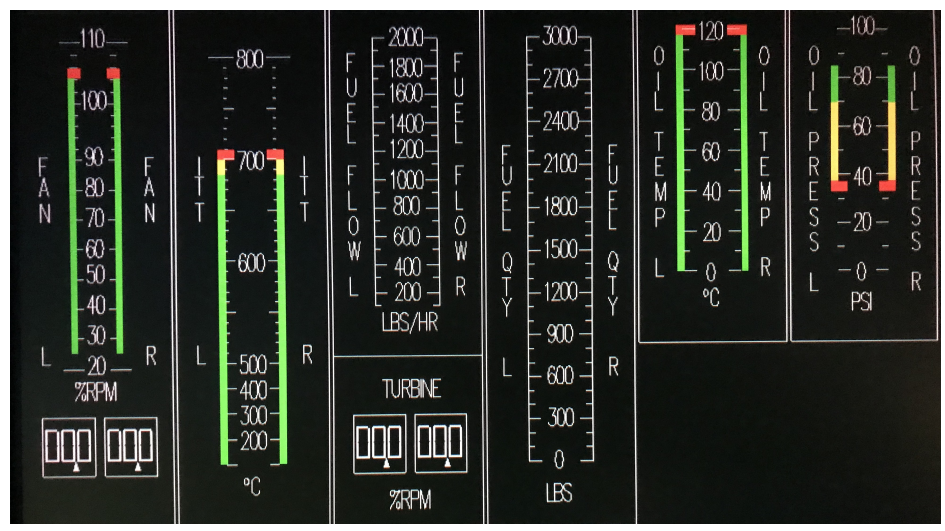

Figure E.4: Engine display

\section{E.2.2. Independent variables}

This experiment contains of one independent variable, being the model that the pilot is using to fly the aircraft. This independent variable has two conditions, with both options represented in the block diagram in Figure E.5 and displayed in Table E.1. The experiment controller can chose to either send the response from the CAP reference model directly to the HMI lab simulation or to send the response from the CAP reference model including controller and aircraft dynamics. Ideally, the pilot does not feel any difference between the two systems. In this case, the functionality of the controller is confirmed, in which case it can be implemented in the aircraft.

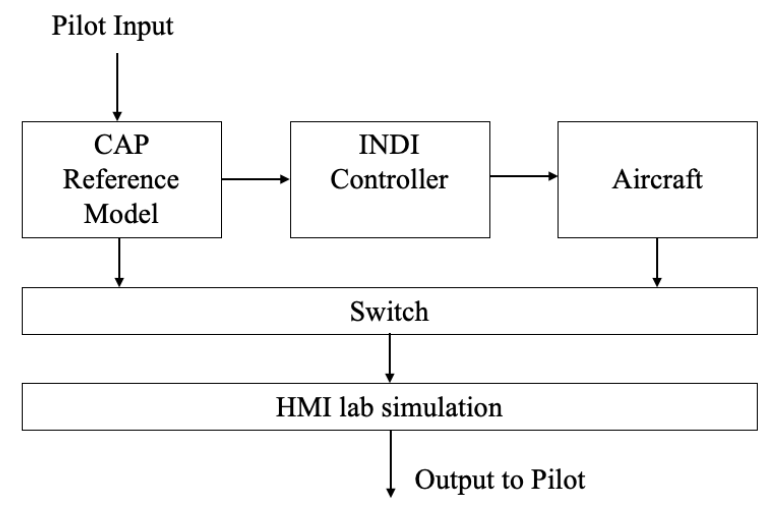

Figure E.5: Flow diagram concerning the independent variable with two conditions

Table E.1: Model independent experimental condition

\begin{tabular}{|c|c||c|c|}
\hline \multicolumn{2}{|c|}{ Condition } & Model \\
\hline \hline$\#$ & Name & Full model & CAP reference model \\
\hline 1 & FULL & $\mathrm{X}$ & - \\
\hline 2 & CAPR & - & $\mathrm{X}$ \\
\hline
\end{tabular}




\section{E.2.3. Dependent variables}

The dependent variables are the variables that are measured to determine the differences between the independent variables. Next to this, they are also used to answer the second research question, where it is required to find the limits of the full model based on the actuator saturation. The dependent variables are defined as follows:

- Cooper-Harper Rating Scale: The subjects had to determine the Cooper-Harper rating after every run. The difference between the CAP reference model and the full model for the same initial condition is used to compare the results.

- Performance: The total time within specified $10 \%$ bandwidth around the required rate of climb is used as performance indicator. This is expressed by the percentage of the total run time referred to as the performance ratio.

- Control activity: An increased workload indicates the pilot has a harder time flying the aircraft. The control activity during the simulation can therefore be used as workload measure. The control activity is measured by the the variance of the input of the pilot. Since the main goal is to determine if the pilots notice the change in longitudinal handling qualities, only the control activity in pitch is of interest.

- Actuator saturation: Actuator saturation is measured since a correlation is expected between the actuator saturation and the differences in Cooper-Harper rating between the different models. Two different flags are used. The first one is logged when actuator saturation occurs and will be referenced as saturation flag in the remainder of this thesis. The second one is logged when actuator saturation occurs and the requested input of the pilot is in the opposite direction of the current aircraft movement. In other words, a pilot might not feel saturation when the aircraft is moving in the direction as requested by the pilot. This flag is referenced to as the combined flag.

- Control question: After every run, it is asked how the handling qualities compare to the previous run. Is the control task easier, harder or about the same?

\section{E.2.4. Initial conditions}

The initial conditions are selected as represented in Figure E.6. These initial conditions are based on pretesting, where it was found that actuator saturation occurs within this region of test points for the given control task. All initial conditions will be tested for both the CAP reference model and the Full model.

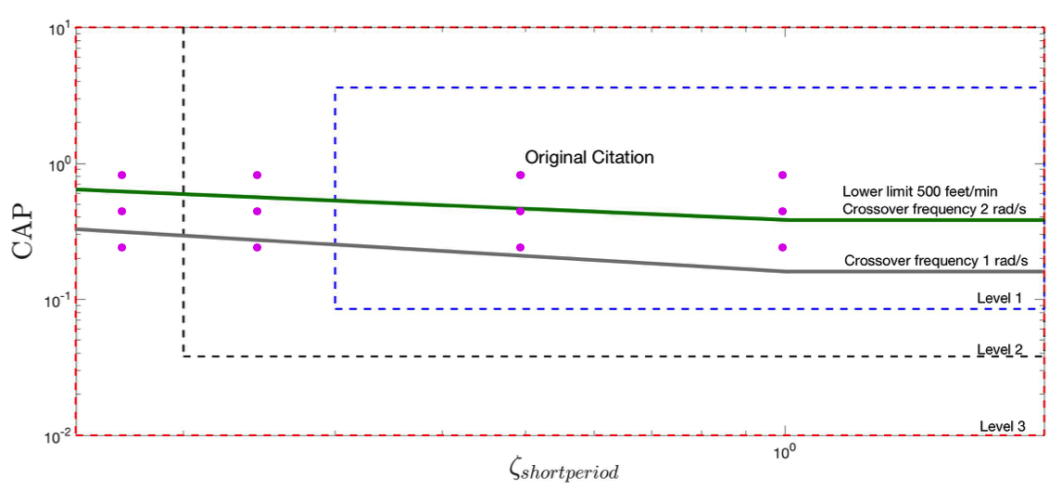

Figure E.6: Initial CAP and Damping conditions set in the CAP reference model 


\section{E.2.5. Experiment matrix and Participants}

As presented in the previous section, there are 12 initial conditions that should be tested for both the CAP reference model and the full model. The initial conditions will be tested in sets of 3 , keeping the damping constant. This procedure has been chosen to minimise the amount of familiarisation runs that have to be performed, since a change in damping has a bigger influence on the handling qualities than the changes in CAP. First the highest CAP condition will be tested, after-which the middle condition and finally the lowest CAP condition are tested. This order is chosen, since it is expected that actuator saturation is reached earlier at lower CAP values. This means the chance of actuator saturation increases over the different test conditions per damping value.

To ensure a proper distribution over the different conditions during the experiment, it is required to test 8 subjects. It would be beneficial to test even more subjects to ensure normality and sphericity during the statistical analysis of the performance and the control activity, but due to limitations in the amount of available pilots, a group of 8 has been selected. This amounts to the experiment matrix as depicted in Figure E.7. The participants are all pilots and given in Table E.2.

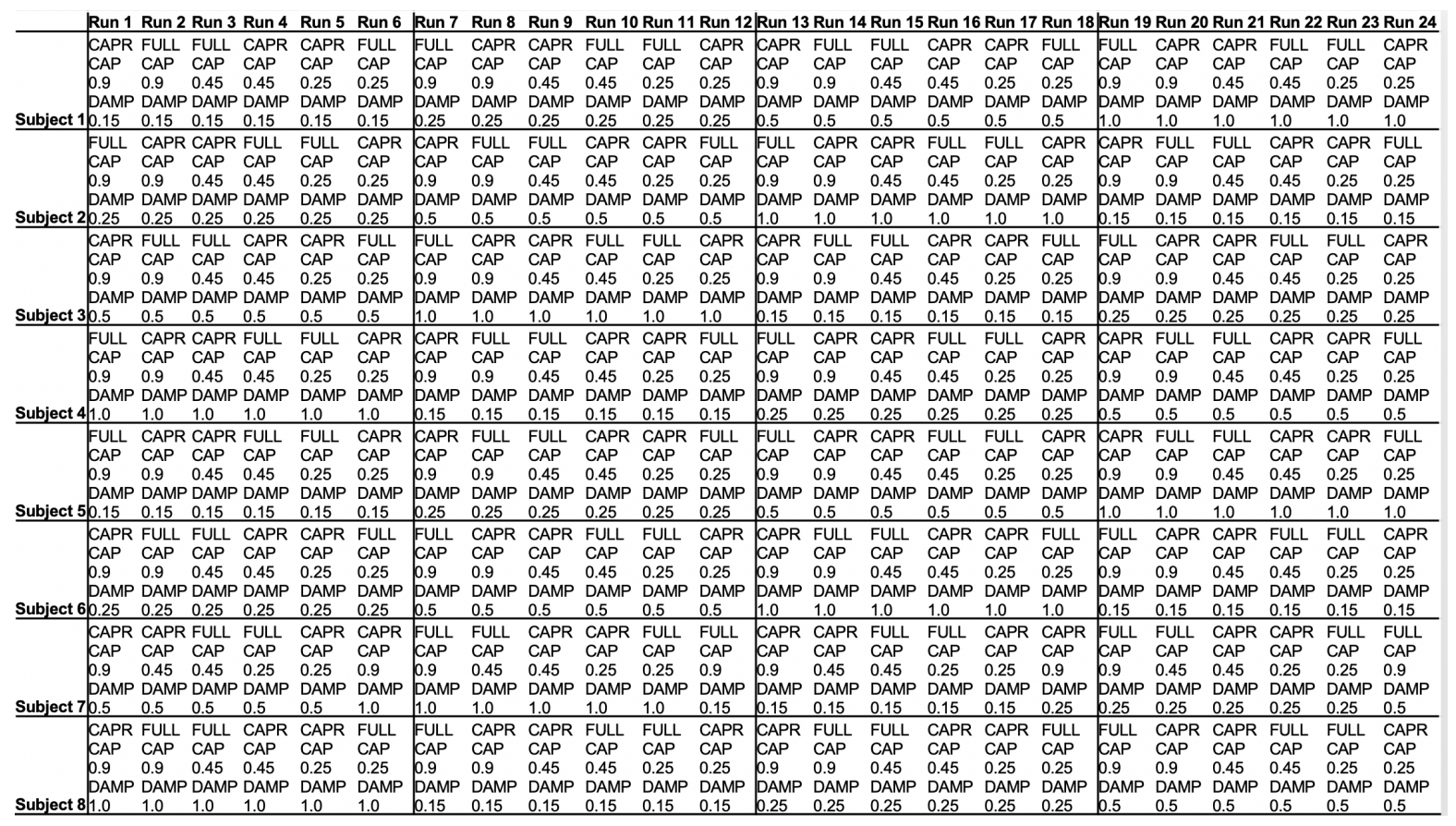

Figure E.7: Experiment matrix showing different test conditions per run per subject

\section{E.2.6. Experimental procedures}

A general overview of the complete experimental procedures are given below:

- Welcoming the test subject (5 minutes)

- Explaining the experiment using the instruction manual send earlier to the test subject (10 minutes)

- Familiarisation run using the settings from the first run from the first set. This is done until the participant feels familiar with the handling qualities of the aircraft (10 minutes)

- First set of runs is performed (15 minutes)

- Small break as long as required (2 minutes)

- Second familiarisation run using the settings from the first run from the second set (10 minutes)

- Second set of runs is performed (15 minutes)

- Small break as long as required (2 minutes)

- Third familiarisation run using the settings from the first run from the third set (10 minutes)

- Third set of runs is performed (15 minutes) 
- Small break as long as required (2 minutes)

- Fourth familiarisation run using the settings from the first run from the fourth set (10 minutes)

- Fourth set of runs is performed (15 minutes)

- Experiment wrap up (5 minutes)

This gives a total experiment time of around 126 minutes. This gives 24 minutes of possible delay time if 2,5 hours of experiment time is communicated to the participant.

Table E.2: Characteristics of the pilot subjects

\begin{tabular}{|c|c|c|c|c|}
\hline Pilot & Age & Sex & Hours & Types of aircraft \\
\hline A & 52 & $\mathrm{M}$ & 12,000 & $\begin{array}{l}\text { Single engine; B777; B787; } \\
\text { Cessna Citation II }(1,000 \mathrm{~h})\end{array}$ \\
\hline B & 63 & M & 20,000 & $\begin{array}{l}\text { Single engine; B777; B787; } \\
\text { Cessna Citation I (500h) }\end{array}$ \\
\hline $\mathrm{C}$ & 59 & M & 3,700 & Single engine; F5; F16 \\
\hline $\mathrm{D}$ & 65 & M & 22,000 & $\begin{array}{l}\text { Single engine; B737; B747; } \\
\text { Cessna Citation I (50h) }\end{array}$ \\
\hline $\mathrm{E}$ & 42 & M & 3,700 & $\begin{array}{l}\text { Single engine; Cessna Cita- } \\
\text { tion II }(1,650 \mathrm{~h})\end{array}$ \\
\hline $\mathrm{F}$ & 46 & M & 2,200 & $\begin{array}{l}\text { Single engine; SA266/277; } \\
\text { Cessna Citation II }(1,300 \mathrm{~h})\end{array}$ \\
\hline G & 58 & M & 14,300 & $\begin{array}{l}\text { Single engine; Jet engine; } \\
\text { Cessna Citation II }(3,000 \mathrm{~h})\end{array}$ \\
\hline $\mathrm{H}$ & 52 & $\mathrm{M}$ & 1,300 & $\begin{array}{l}\text { Single engine; Jet engine; } \\
\text { Cessna Citation II ( } 6 \mathrm{~h})\end{array}$ \\
\hline
\end{tabular}

\section{E.2.7. Hypotheses}

The hypotheses are defined as follows:

- H.1: The performance of the pilots is similar for both models if no actuator saturation is occurring. Performance is expected to be lower if pilots reach the saturation limits of the actuators. Since actuator saturation is expected during the experiment, the full model is likely to have lower performance compared to the CAP reference model.

- H.2: The control activity is higher for the full model, since the full model includes actuator saturation. This means that pilots will sometimes not experience the expected response from the aircraft.

- H.3: Pilots will give more similar Cooper-Harper ratings for a higher CAP, since the offline simulation showed that actuator saturation time is lower for these conditions. However, this depends on the crossover frequency of the pilot and the assumption that pilots only experience differences when a saturation time of more than 0.2 seconds occurs.

\section{E.3. Experiment instruction manual}

The experiment instruction manual that has been handed out to the experiment participants is given in this section on the next few pages. 


\title{
Instruction manual: \\ Variable Stability In-Flight Simulation Experiment
}

\author{
P.A. (Pepijn) Scholten, 4214099 \\ MSc Control \& Simulation
}

December 15, 2018

\section{Dear participant,}

First of all I would like to thank you for participating in this experiment regarding Variable Stability In-Flight Simulation. The goal of this experiment is to asses how you as a pilot can identify the different handling qualities when flying the simulator. The experiment will be performed in the Human Machine Interface (HMI) lab as can be seen in Figure 1.

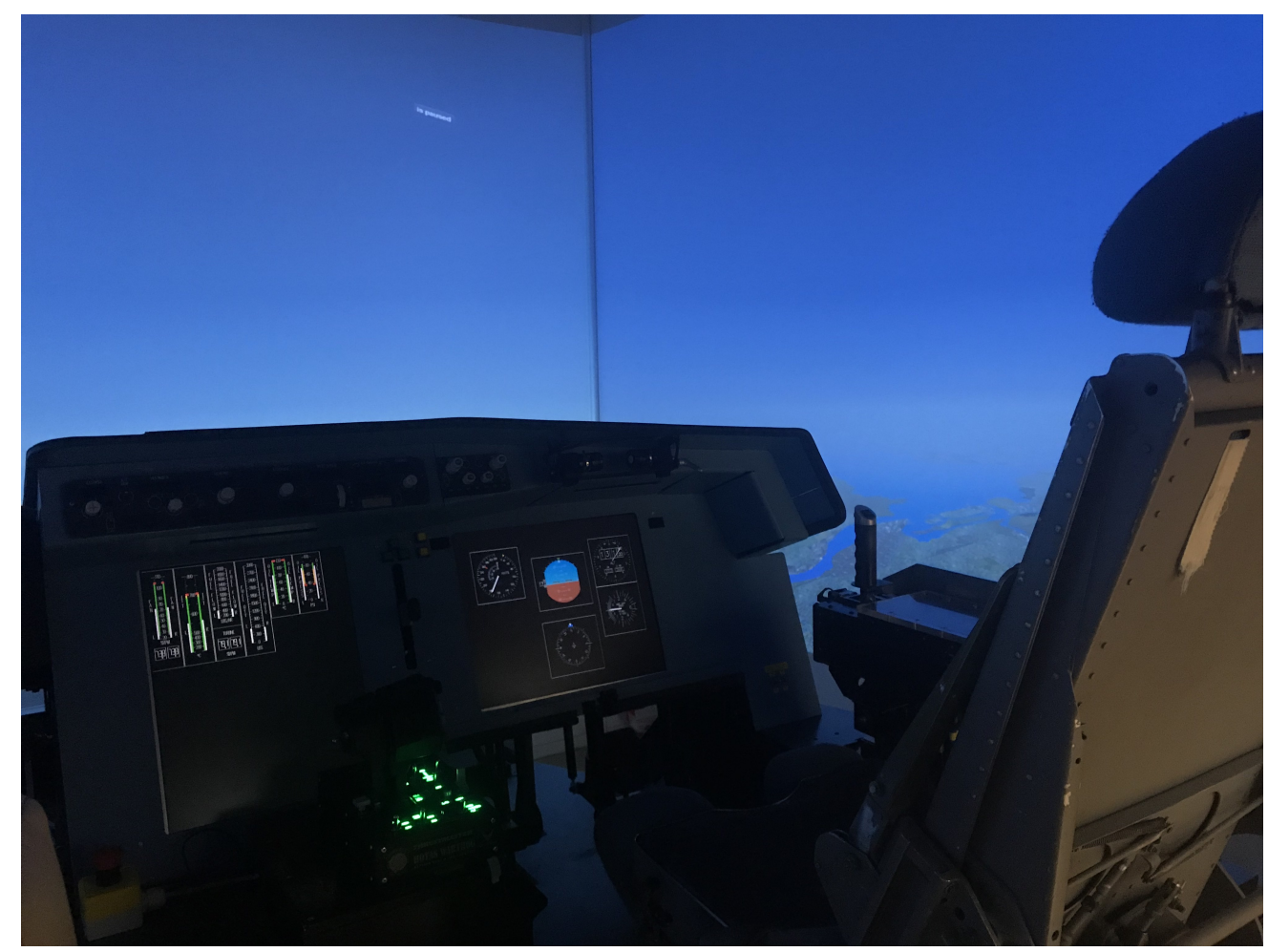

Figure 1: HMI lab

The experiment consists of 24 runs in total, which are divided into 4 sets of 6 runs. Each set will start with a familiarisation phase, where you can get familiar with the experiment set up and the handling qualities of the aircraft. Each run will last about 2 minutes. After the run you will be handed a short questionnaire regarding that specific run and you will have to give a Cooper-Harper rating for the handling qualities of that specific run. Between each set of runs there is room for a small break to rest upon your request. 


\section{Control task}

Your control task will be the same during every test. The aircraft has to be controlled to a Rate of Climb of $1000 \mathrm{feet} / \mathrm{min}$ and $-1000 \mathrm{feet} / \mathrm{min}$. These conditions will be changed every ten seconds for a total of 10 changes for each run upon the command of the experimenter. Your task will be to adapt to this change as quickly as possible. This control task is chosen, since the many changes in Rate of Climb make it relatively easy for you to notice the differences in Handling Qualities. The vertical speed or Rate of Climb is displayed on the Vertical Speed Indicator (VSI) as depicted in Figure 2.

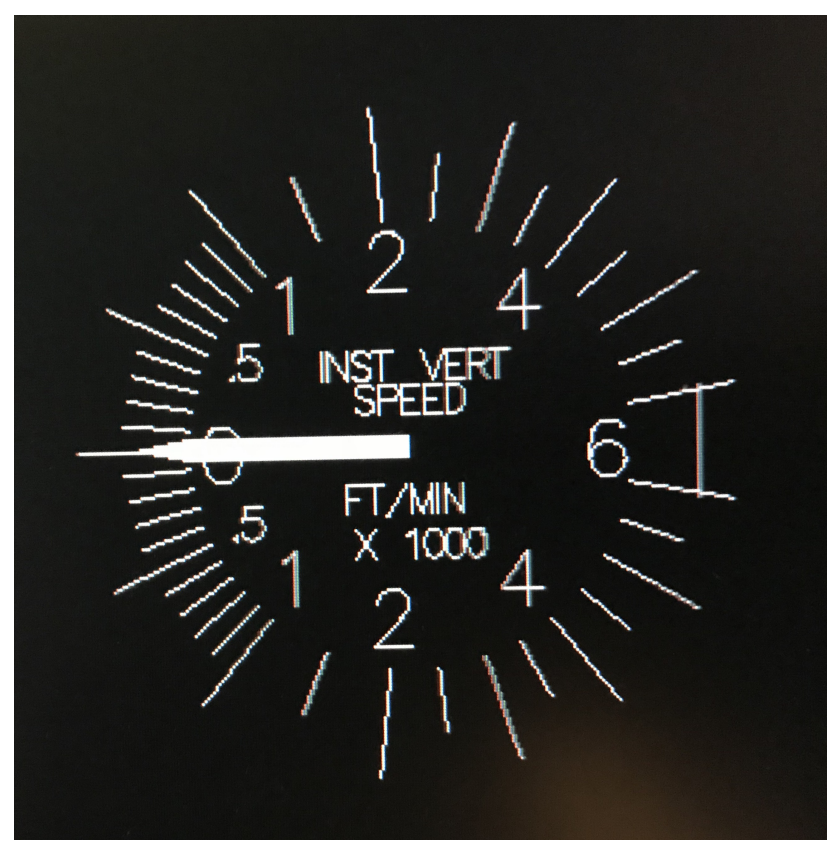

Figure 2: Vertical Speed Indicator flight display

The initial condition that you start at is chosen is at 4000 meters altitude and a true airspeed of $110 \mathrm{~m} / \mathrm{s}$. This initial condition is chosen since the preliminary research of this thesis has shown that within this condition, the best performance can be achieved regarding variable stability. You will start at a Rate of Climb of zero, after-which the switching will start on the command of the experimenter. Note that the first Rate of Climb that is commanded will be negative $(-1000 \mathrm{feet} / \mathrm{min})$.

\section{Controlled element}

For generating control inputs, a side stick is used. This side stick only controls the roll and pitch axes. The stick is a force-stick, which means that it physically does not deviate from its position, but it passes through your input based on the amount of force that is exerted in a specific direction. This is comparable to the force-stick that is present within the Cessna Citation II aircraft. Side-slip is automatically controlled to zero so you don't have to worry about this.

Because of the nature of the control task, it is not required for the pilot to control the speed. The speed will remain close to the initial condition, due to the relatively quick variations in Rate of Climb, both positive and negative. This makes it easier for you to focus on the Rate of Climb task only. 


\section{Displays and feedback}

The experiment will be held within the HMI lab at the faculty of Aerospace Engineering. Preferably, this experiment would have been executed in the SIMONA full motion research simulator to also provide motion feedback, but sadly the SIMONA is in maintenance during the time of this writing.

You will receive the information of the state of the aircraft using the primary flight display as given in Figure 3. You will mainly be focusing on the Vertical Speed Indicator as presented in the previous section, since the control task is based on this display. The information regarding the state of the engines and the fuel flow is depicted as shown in Figure 4. These displays are chosen to mimic the real scenario in the Cessna Citation II as closely as possible.

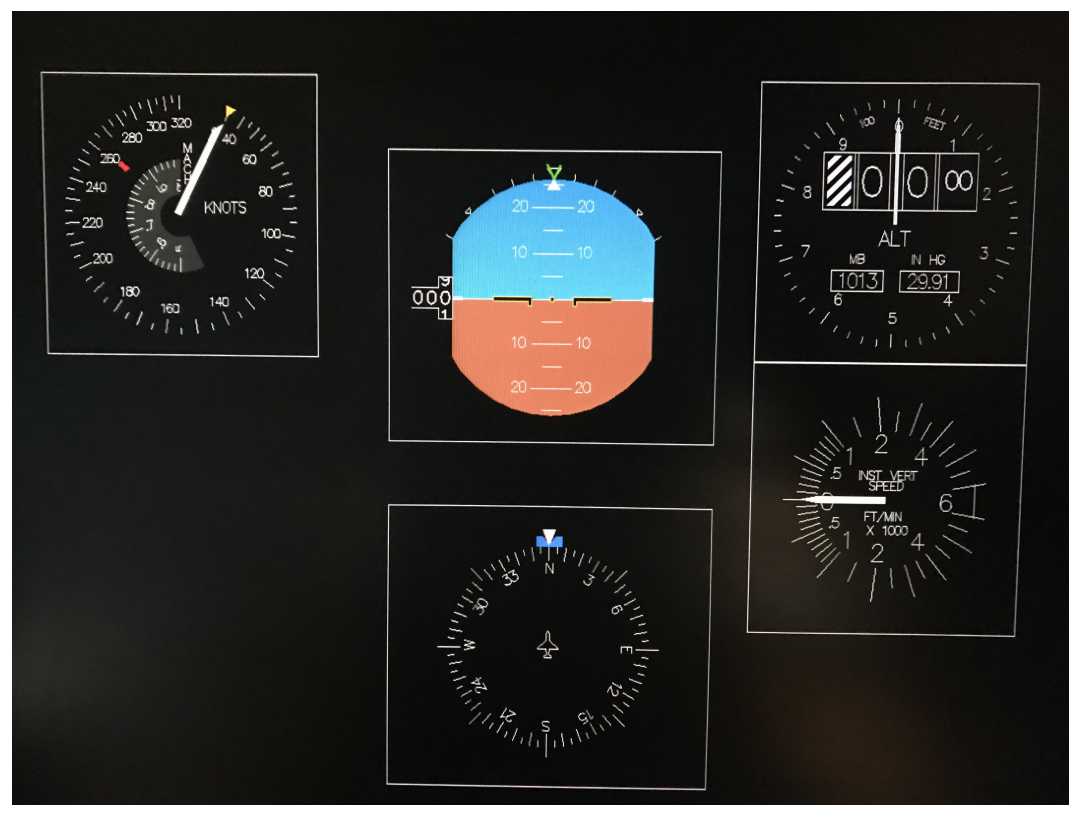

Figure 3: Primary flight display

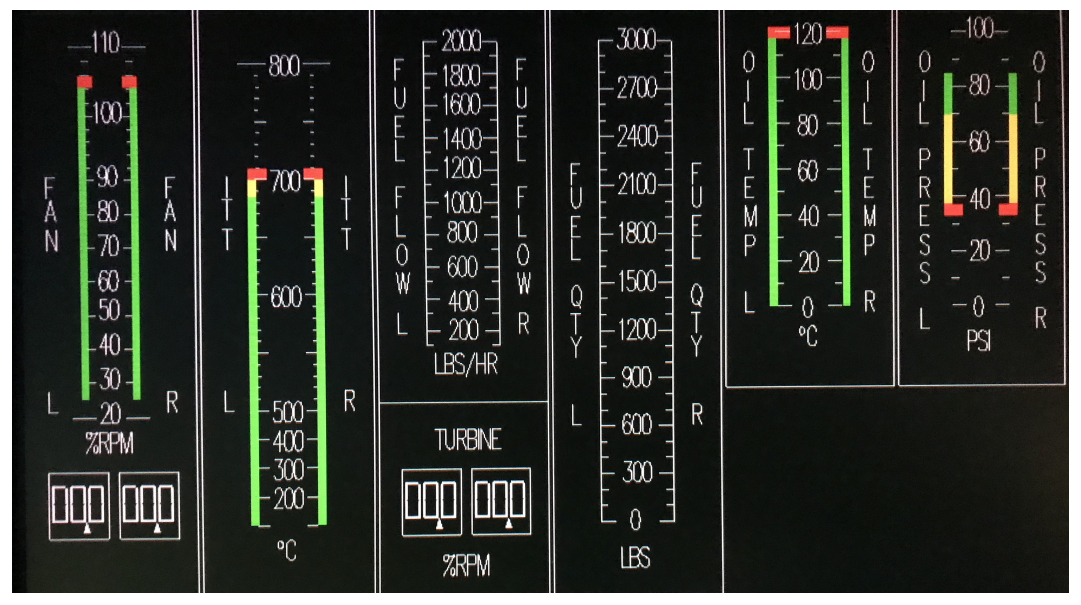

Figure 4: Engine display 


\section{Cooper-Harper rating scale}

The Cooper-Harper rating scale is the accepted standard for subjective handling quality measurement. Cooper and Harper [1] developed a decision tree which uses a rating scale to assess the aircraft handling quality. The scale takes uses comments from pilots and translates them into a quantitative measure. The decision tree can be found in Figure 5. You will be asked to determine the Cooper-Harper rating after every run.

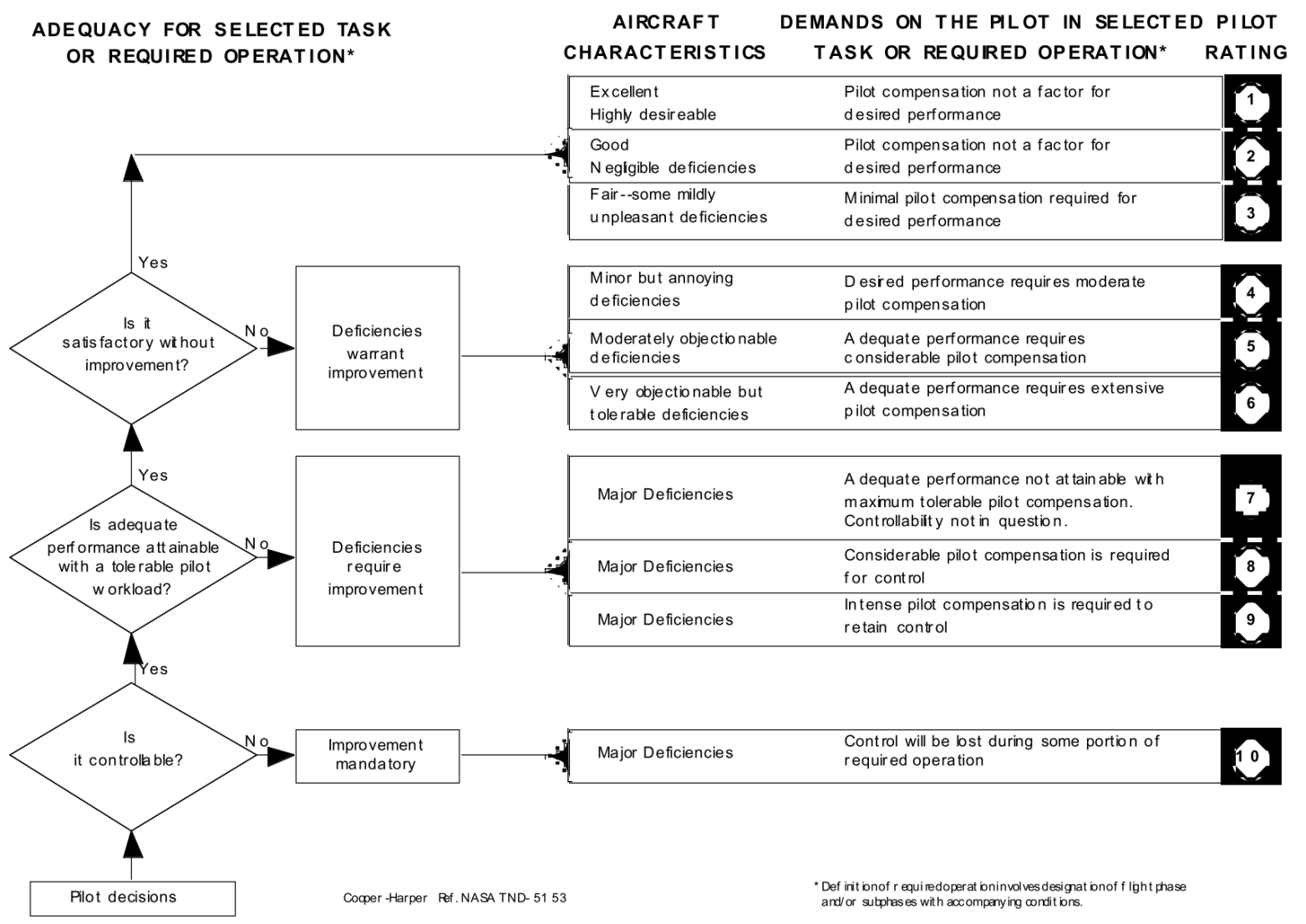

Figure 5: Cooper-Harper rating scale decision tree [1] 


\section{Questionnaire}

After each run you will receive the following questionnaire. You will be asked to determine the relative difference in handling qualities compared to the previous run. Is the aircraft easier to control, worse to control or about the same? The scale is from 1-7, so you should give a 4 for about the same, a 7 for a lot easier and a 1 for a lot worse. This scale is depicted in Figure 6.

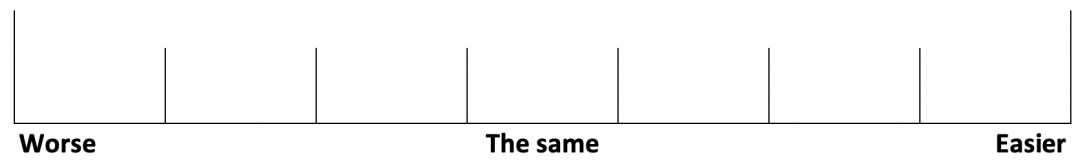

Figure 6: Questionnaire rating scale relative controllability compared to previous run

\section{Experiment wrap-up}

During the experiment wrap-up you will be asked a couple of questions regarding the experiment itself. This is of importance to future students who might want to improve upon the experiment or the HMI lab itself. 


\section{References}

[1] G. Cooper and R. Harper. Handling qualities and pilot evaluation. Journal of Guidance, Control, and Dynamics, 9(5):515-529, 1986. 


\section{Post-Experiment Questionnaire}

Please fill in the following post-experiment questionnaire.

Subject ID:

Date:

1. How much experience do you have as a pilot? And how much flying the Cessna Citation II? Preferably an estimate of your flight hours

2. Did you have any previous experience with Handling Qualities experiments and the Cooper-Harper rating scale?

3. What did you think of the realism of the simulator on a scale of 1-5? Please mark only one oval: $1 \mathrm{O} \quad 2 \mathrm{O} \quad 3 \mathrm{O} \quad 4 \mathrm{O} \quad 5 \mathrm{O}$

4. Do you have any suggestions for improvement of the simulator?

5. Do you have any other comments regarding the experiment? 


\section{E.4. Experiment script \\ Dear participant,}

First of all I would like to thank you for participating in this experiment regarding Variable Stability In-Flight Simulation. The goal of this experiment is to asses how you as a pilot can identify the different handling qualities when flying the simulator. The experiment will be performed in the HMI lab as can be seen in front of you.

- Did you manage to read the instruction manual? In both cases still quickly discuss the instruction manual.

- Do you understand what we are going to do?

- Take a seat: We will start with the first familiarisation run when you are ready.

- Familiarisation runs X: Repeat until test subject feels comfortable. Minimum of 3-4 times Are you comfortable with the aircraft dynamics and the given control task? If YES continue.

- Check data logging

- First set of runs X: Are you ready? I will count down from 3 and then I say 'start'. When I say start, the run will begin. After every 10 seconds I will say 'yes'. At this point you have to change the control task. Continue until end of runs

- Check data logging

- Finish above loop of Familiarisation and Normal Runs until experiment is complete

- Fill in post-experiment questionnaire. Do you have any other comments about the experiment?

Thank you for your participation. I will send the results of the experiment to you after I finished data processing. Do you have any other questions? 


\section{E.5. Additional results experiment}

The results that did not make the paper are given in this section. Firstly, the post-experiment questionnaire is discussed. Secondly, additional plots are displayed. It should be noted that the control question was removed from the results, since the Cooper-Harper ratings themselves already provided enough information regarding the differences in handling qualities experienced by the pilots.

\section{E.5.1. Post-experiment questionnaire}

This subsection displays the responses to the post-experiment questionnaire. They are sorted per test-subject. Firstly, the questions are repeated, after which the results are given.

\section{Questions}

1. How much experience do you have as a pilot? And how much flying the Cessna Citation II? Preferably an estimate of your flight hours

2. Did you have any previous experience with Handling Qualities experiments and the Cooper-Harper rating scale?

3. What did you think of the realism of the simulator on a scale of 1-5? Please mark only one oval: $1 \mathrm{O} 2 \mathrm{O} \quad 3 \mathrm{O} \quad 4 \mathrm{O} \quad 5 \mathrm{O}$

4. Do you have any suggestions for improvement of the simulator?

5. Do you have any other comments regarding the experiment?

\section{Subject 1}

1. 12,000 flight hours, of which 1,000 hours on the Citation C550 (II).

2. Yes

3. 2

4. Well suited for this task

5. Learning seems important

\section{Subject 2}

1. 20,000 flight hours, of which 100 hours on the Citation C500 (I).

2. No

3. 4

4. No

5. No

\section{Subject 3}

1. 3,700 flight hours, of which 400 on the F-5, 2,800 on the F-16, with the remainder on other aircraft. Zero hours on the Citation.

2. Yes, at Test Pilot school, F-16 Handling Qualities testing and various other aircraft.

3. 4

4. Full motion would have been better, especially in the low damped test conditions, but this was workable.

5. It was fun! 


\section{Subject 4}

1. 22,000 flight hours, of which 50 in the Citation 500 (I). 21,200 in multi-engine jet, 800 in single engine prop.

2. No.

3. 5

4. -

5. Nice and interesting!

\section{Subject 5}

1. 3,700 flight hours, of which 1,650 in the Cessna Citation II.

2. Yes, limited experience during experiments

3. 4

4. PFD/MFD higher with respect to the side stick placement

5. No.

\section{Subject 6}

1. 2,200 flight hours, of which 1,300 in the Cessna Citation II.

2. Yes.

3. 4

4. No.

5. Asymmetric control from + to - Rate of Climb, I recognised that the controller is effectively an attitude hold mode controller.

\section{Subject 7}

1. 14,300 flight hours, of which 3,000 in the Cessna Citation II.

2. No.

3. 5

4. No

5. Nice to participate!

\section{Subject 8}

1. 1,300 flight hours, of which 6 hours on the Cessna Citation II.

2. No.

3. 4

4. Remove cross-coupling of sidestick.

5. - 


\section{E.5.2. Additional plots}

Some additional plots were generated but not used in the paper. Figure E.8 sorted the combined flag by CAP and damping ratio. However, only 8 data points per damping ratio for that specific CAP was not sufficient to draw any significant conclusions. Figure E.9 and Figure E.10 were not included since they were considered to be too detailed. Figure E.11 was not included in the paper, since the maximum flag time provided a clearer distinction by Cooper-Harper rating compared to the counted version.
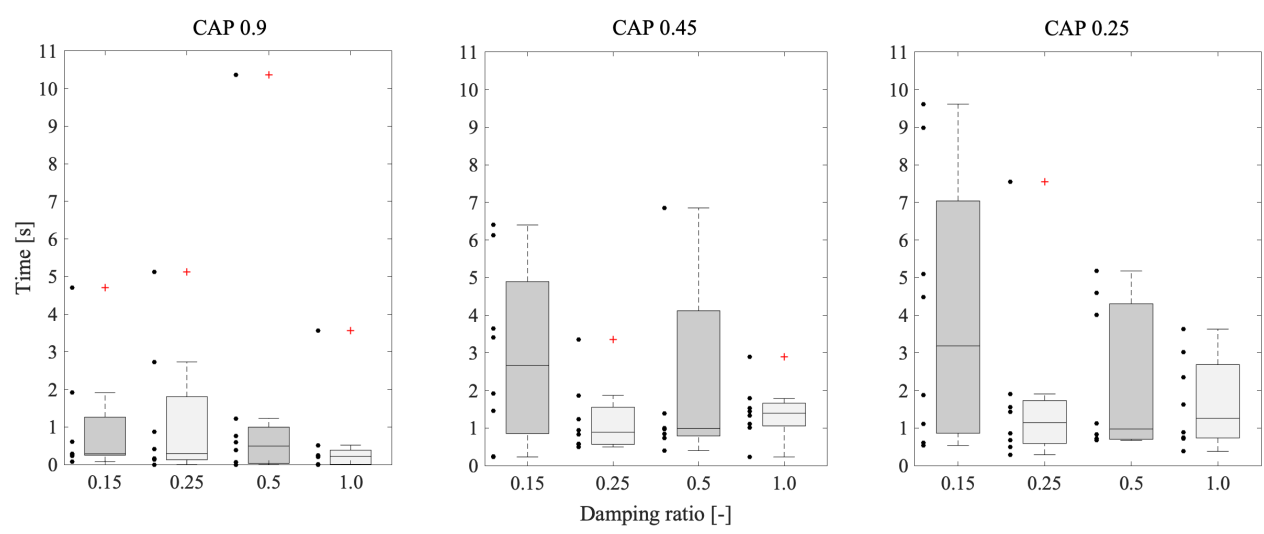

Figure E.8: Means and the 95\% confidence limits of the combined flag sorted by CAP and Damping ratio

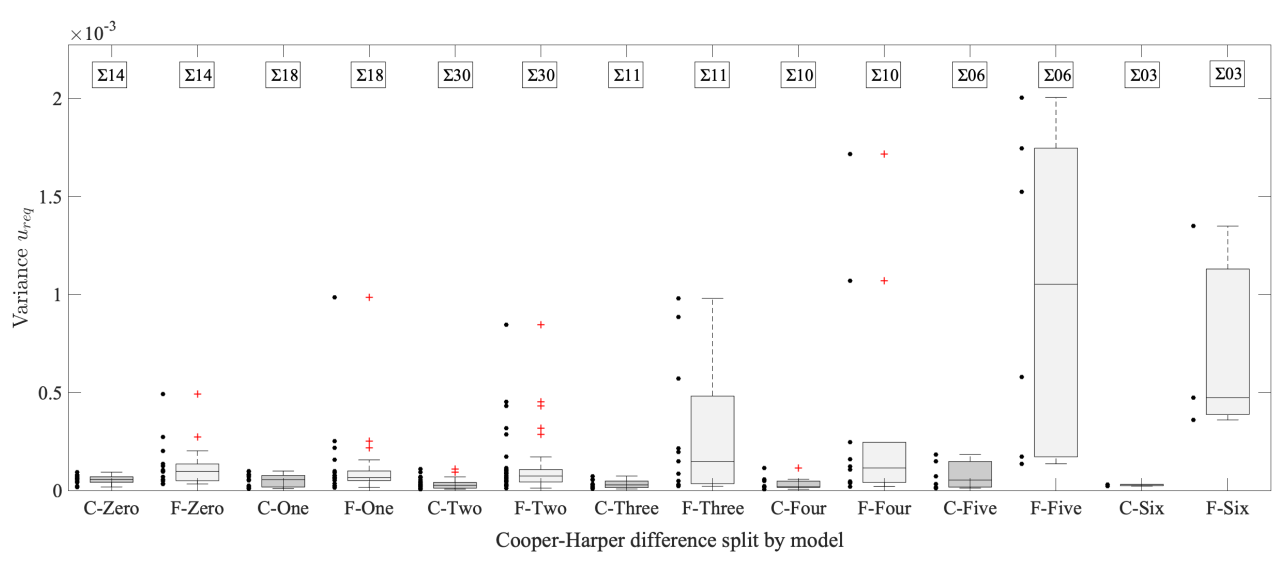

Figure E.9: Means and the 95\% confidence limits of the control activity sorted by model and Cooper-Harper difference $(\mathrm{C}=\mathrm{CAP}$ reference model; F = Full model) 


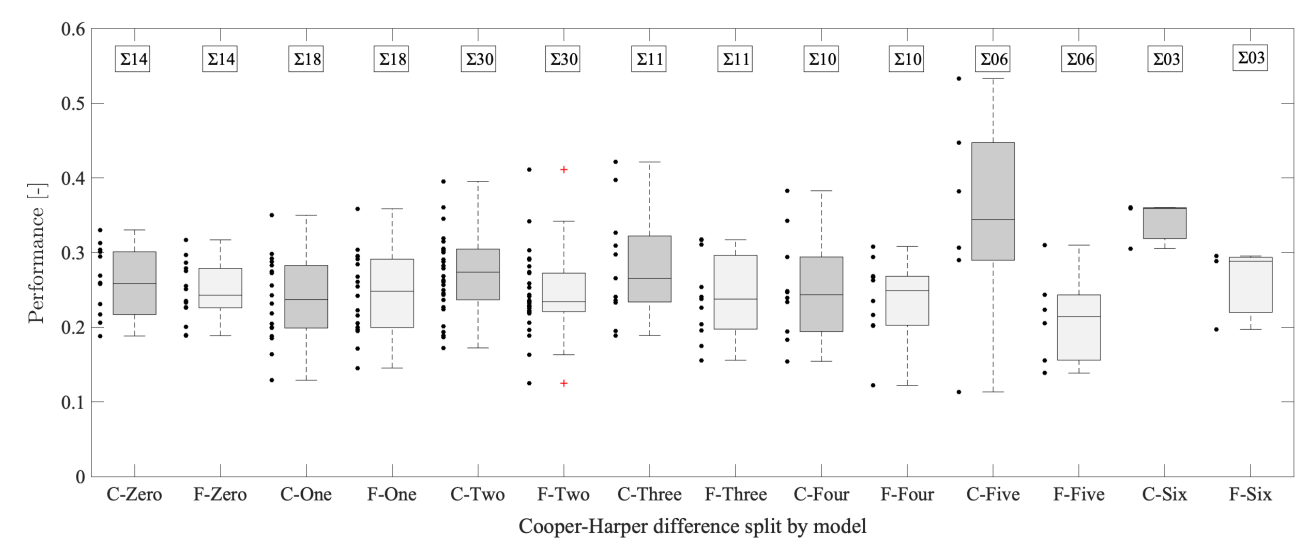

Figure E.10: Means and the 95\% confidence limits of the task performance sorted by model and Cooper-Harper difference $(C=C A P$ reference model; F = Full model)

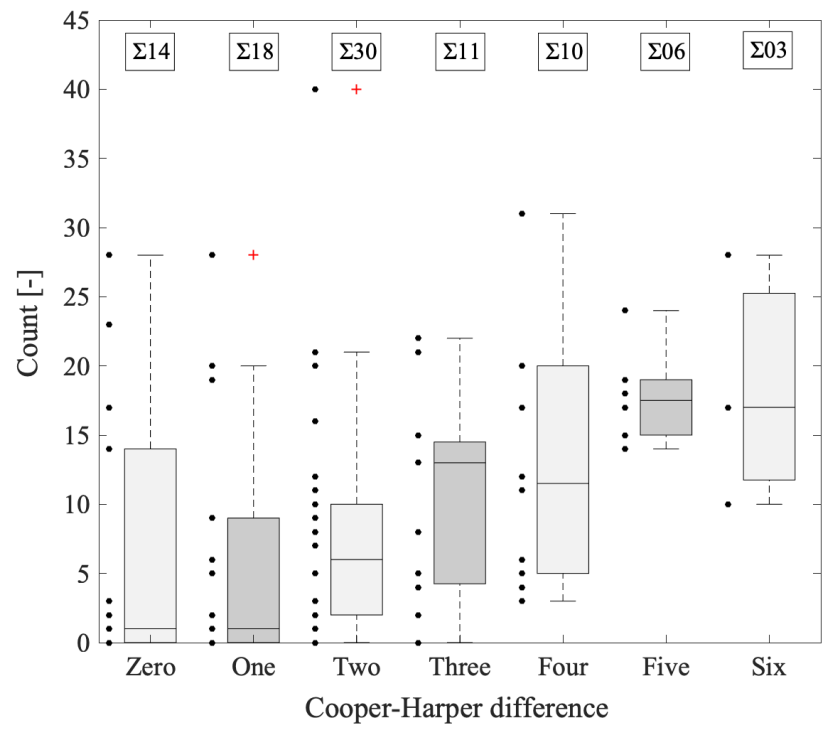

Figure E.11: Means and the 95\% confidence limits of the combined flag with counted flag duration larger than 0.2 seconds 



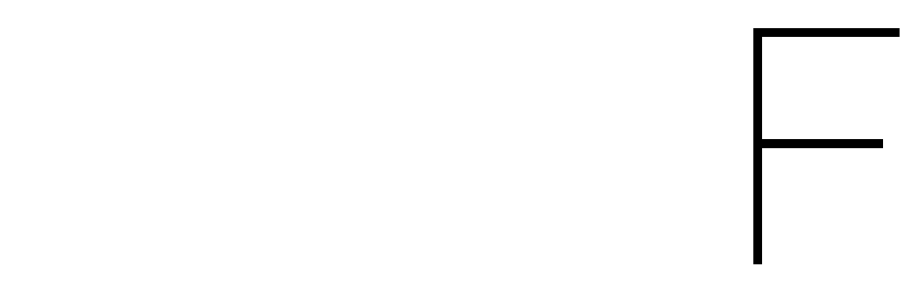

\section{Experiment II: Flight test}

To validate the controller design and implementation, four flight tests were performed over the course of eight days. The flights all departed and terminated at Amsterdam Schiphol Airport (AMS) and were flown in the TMA Delta and above the North Sea, within the Dutch Airspace. The system is validated using the three initial flight tests, whereas the final flight was a demonstration flight.

\section{F.1. Changes to the controller}

Multiple changes have been made to the controller with respect to the simulator version. The complete system dynamics are removed and changed to ensure that they only output the moment effectiveness matrix. All other outputs were received from sensors based on the aircraft itself. The requested deflection that comes from the INDI controller was linked to the autopilot servos from the aircraft's fly-by-wire system.

New angular accelerometers were installed on the Cessna Citation II laboratory aircraft. These were an addition to the previously available angular accelerations which were established by differentiating the angular rates. Second order filters were introduced to these angular accelerations to remove noise whilst still maintaining the original signal without too much time delay. Especially the differentiated angular rate signals consisted of a relatively high noise.

The controller required a conversion of the stick gains since the stick on the aircraft was not identical to the electrohydraulic stick in the fixed-base simulator. The stick on the aircraft converted the input from the pilots to a different scale, thus the gains were adapted to maintain the same scaling as in the simulator.

In comparison to the fixed initial conditions in the fixed-based simulator, the initial conditions depend on the actual flying conditions upon experiment initiation. These initial conditions were continuously tuned when the experimental control mode was not enabled. The updated initial conditions were also used in the determination of the moment effectiveness matrix.

The fixed-based simulator experiments are based on the DASMAT model, which consisted of an inertia tensor based on the Cessna Citation I. Since the experiment is conducted in the Cessna Citation II, it was required to update this inertia tensor based on the newly developed model by Van den Hoek et al. [34]. The model mismatch between the DASMAT model and the new model was highest for the inertia around the y-axis $I_{y y}$, with a 30\% mismatch respectively. This update improved the final performance of the controller. 


\section{F.2. Validation plan}

\section{F.2.1. Apparatus}

The Cessna Citation II business jet, co-owned by the Delft Aerospace Engineering faculty, was used in the flight tests (Figure F.1). The flight tests are performed in a clean configuration with spoilers, gear and flaps retracted. The angle of attack and sideslip are measured in clean air using a boom mounted in front of the aircraft. The automatic trim influences the handling qualities of the aircraft, and is therefore deactivated during the experiment. The aircraft was controlled by the co-pilot using the sidestick. The other pilot was the safety pilot during the experiment. The experimenter controlled the software and its variable stability settings via a wireless connection using a laptop in the cabin.

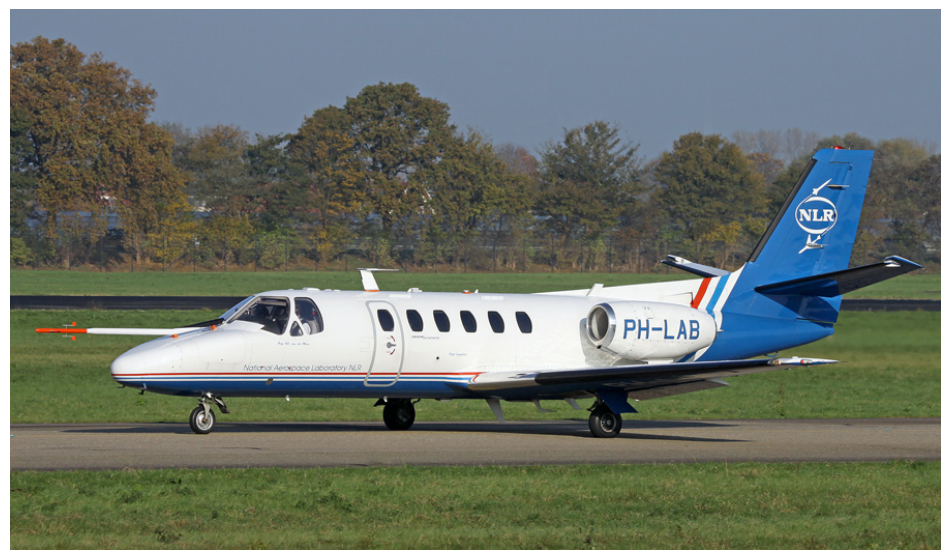

Figure F.1: Cessna Citation II laboratory aircraft with boom configuration

\section{F.2.2. Procedure}

The experiments in pitch focused mainly on the longitudinal handling qualities, similar to the experiment in the simulator. In addition, a number of roll manoeuvres were performed to test the differences in roll time constants. In addition, to demonstrate the system potential to be used in pilot training, the effect of roll time constants stick time delays and stick gains are investigated.

Two initial conditions were used during the different flight tests. Both conditions were at an altitude of FL130, but with a varying airspeed of 175 or 190 knots. To ensure safe execution of the experiment, a strict procedure was implemented during the experiments. First, the safety pilot flies to the requested altitude. Then he trims the aircraft for the desired airspeed in horizontal flight and disables the auto trim. At the same time, the experimenter selects the desired parameter configuration in the DUECA software. Activation of the autopilot based on the controller software requires activation of both the roll and pitch axes. This is achieved by a trigger of both the safety pilot and the experimenter for each axis. Finally, the experimental control mode is initiated by the co-pilot, with 'hands-of-stick' to prevent undesirable initial control inputs.

\section{F.2.3. Subjects and Instruction to Subjects}

To validate the controller by noticing the differences in handling qualities, pilots require experience on the Cessna Citation II laboratory aircraft. Three pilots have flown the aircraft in the experiments, being pilot E, F and G (Table E.2). In contrast to the fixed-based simulator experiments, the pilots do not assign CooperHarper ratings, due to the limited number of experimental runs.

The pilots received instructions for the different phases in flight. In accordance with the simulator task, the pilots are instructed to fly at $\pm 1000 \mathrm{feet} / \mathrm{min}$ rate of climb during the test. However, the pilot is free to decide between ascent and descent given he remains below the FL135. This restriction is required, as a significant reduction of torque in the fly-by-wire system is expected above FL135. During the lateral handling tests, the pilots were asked to assess differences between roll time constants. They decided on capturing a specific roll angle to investigate the performance of the system. 


\section{F.2.4. Testing report}

Some software errors existed in the IMAR IMU which would send not a number values to the controller. This would cause the controller to crash. This issue was resolved before the first test flight. Next to this, a new synchro was installed in the fly-by-wire system which had to be calibrated. The stick was calibrated, since the output from the stick's initial condition was not equal to zero. The PCH was disabled during the flight tests, because this provides the clearest indication of the bandwidth within specific manoeuvres are possible.

During the first test flight, the pilots observed a difference in pitch performance compared to the fixed-based simulator experiment. Similarly, the controller modified the aircraft's damping behaviour, but again it was not as evident as in the simulator. In addition, a high-frequency oscillation occurred when the roll axis was enabled. It is expected that this oscillation occurred because of the sideslip controller, which actively adjusts the roll axis. The combination of the sideslip controller and the yaw-damper of the aircraft's autopilot was expected to conflict. The yaw-damper could not be disabled during flight, whilst it was disabled during the simulator experiment.

For the second test flight, the sideslip controller was modified so it could be disabled during the flight. Even with the sideslip controller disabled, another oscillation existed in roll. However, it appeared to be at a lower frequency compared to the first flight. The time delay and stick gains were also tested during this flight, and the pilots could identify the time delay and changes in stick gains appropriately. A time delay larger than 0.5 seconds caused the pilot to excite pilot induced oscillations (PIO).

When analysing the data from the second flight, it was determined that the angular accelerometer calibration was not performed correctly. In addition, the angular rates that were fed from the aircraft to the linear controller appeared to be incorrect, causing the oscillations in the roll axis. Both issues were fixed before the third flight.

During the third test flight, the pilot commented that the longitudinal response from the aircraft was more similar to the response in the simulator. Different CAP and damping conditions could be differentiated more easily from each other, compared to the previous flights. The oscillation in the roll axis also disappeared, which enabled the testing of the roll time constant for the first time. A smaller time constant introduced undesired overshoot, but pilots could still identify the changes in roll time constant. Lower gain control by the pilots resulted in better performance of the controller.

The final flight was a demonstration flight. The pilots tried to find the limitations of the system. They noted that a change in indicated airspeed of more than $\pm 5[\mathrm{~m} / \mathrm{s}]$ would result in actuator saturation. Furthermore, they noticed that it was difficult to capture a roll angle with full stick input left or right, but easier with lower gain inputs.

\section{F.3. PH-LAB sensor systems}

The sensor characteristics of the Cessna Citation II laboratory aircraft are given in Table F.1. The location of the sensors is given in Table F.2.

Table F.1: Sensor characteristics of the Cessna Citation II Laboratory Aircraft [40] [41] [36]

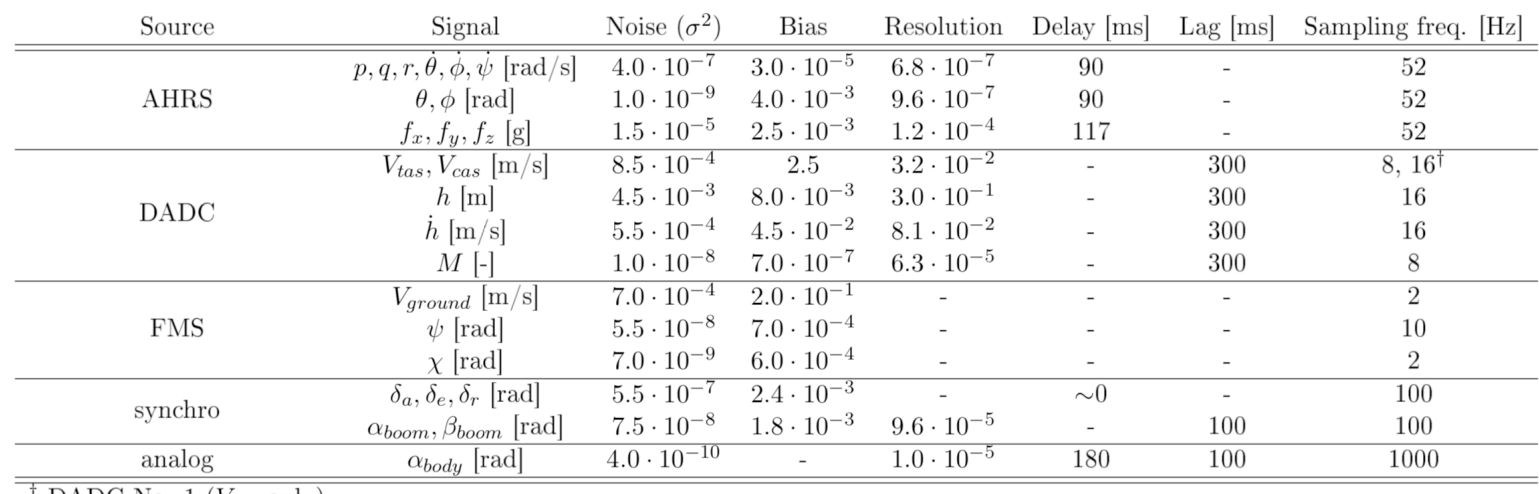

† DADC No. 1 ( $V_{\text {tas }}$ only) 
Table F.2: Location of the sensors on the aircraft [42]

\begin{tabular}{c|ccccc} 
& $f_{x}, f_{y}, f_{z}$ & $\alpha_{\text {boom }}$ & $\beta_{\text {boom }}$ & $\alpha_{\text {body }}$ & $p_{t}$ \\
\hline \hline$x[\mathrm{~m}]$ & 1.6256 & -0.6985 & -0.4140 & 4.4069 & - \\
$y[\mathrm{~m}]$ & \pm 0.3810 & - & - & - & \pm 0.2972 \\
$z[\mathrm{~m}]$ & 2.6670 & - & 3.2639 & - & 2.3114 \\
\hline \hline
\end{tabular}

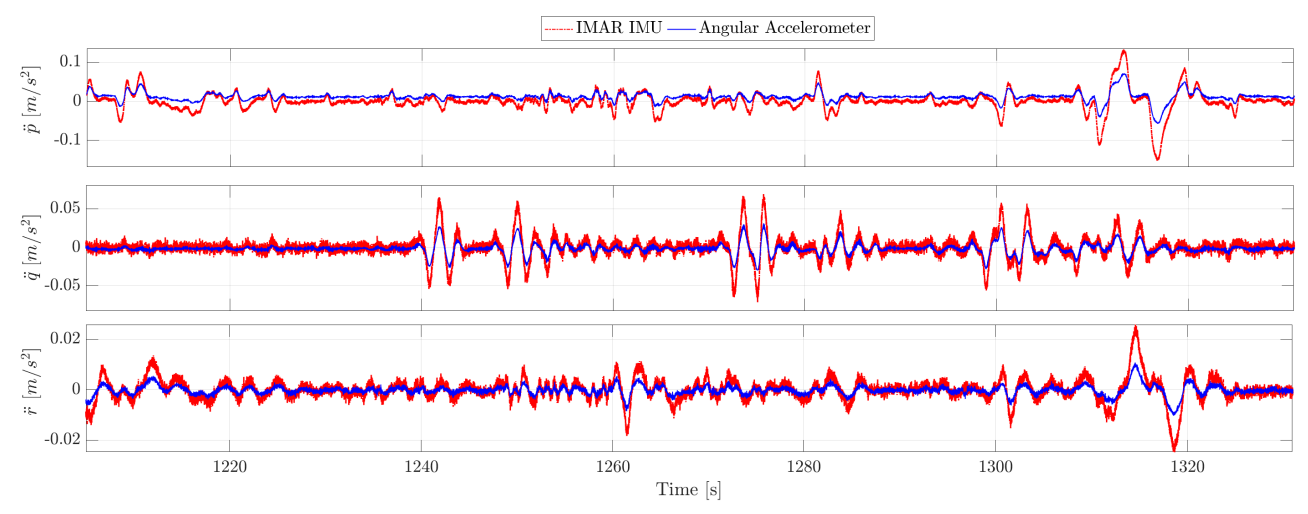

Figure F.3: Comparison of IMAR IMU accelerations and angular accelerometer accelerations

\section{F.4. Additional results}

The unwanted oscillatory behaviour in roll experienced during the third flight is displayed in Figure F.2. It can be seen that the oscillation in roll is sustained even when the pilot is not giving an input anymore. When analysing the data from this flight, it was determined that the angular accelerometer calibration was not performed correctly. In addition, the angular rates that were fed from the aircraft to the linear controller appeared to be incorrect, causing the oscillations in the roll axis. Both issues were fixed before the third flight.
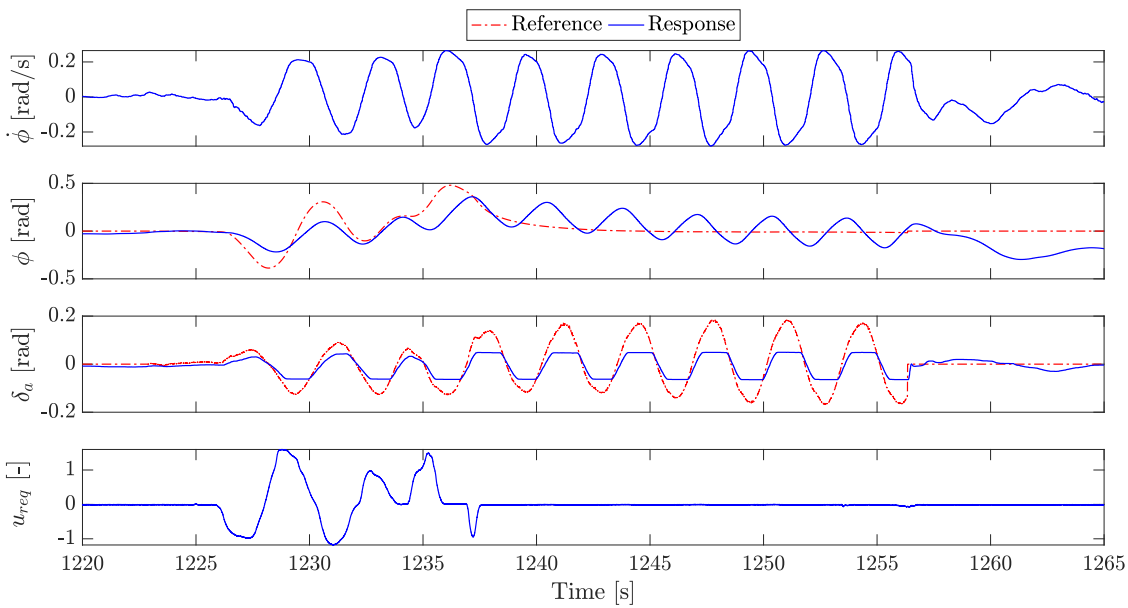

Figure F.2: Oscillatory response of aircraft without additional pilot input

New angular accelerometers are installed in the Cessna Citation II laboratory aircraft before the experiment. Their signal is compared to the original differentiation from the angular rates within the IMAR IMU in Figure F.3. As expected, the noise in the differentiated signal from the IMAR IMU is higher compared to the noise from the angular accelerometers. However, it appears that a gain difference exists between the IMAR IMU and the angular accelerometers. Almost all manoeuvres were flown with the IMAR IMU accelerations, which were also used in other research, so this does not impact the results. However, additional research is required when the angular acceleromters will be used as the new standard. 


\section{Bibliography}

[1] N. C. Weingarten. History of In-Flight Simulation at General Dynamics. Journal of Aircraft, 42(2):290298, 2005. ISSN 00218669. doi: 10.2514/1.4663.

[2] P. Hamel. In-Flight Simulators and Fly-by-Wire / Light Demonstrators. Springer Nature, 2017. ISBN 978-3-319-53996-6.

[3] A. Mirza, M. M. Van Paassen, and M. Mulder. Simulator Evaluation of a Medium-Cost Variable Stability System for a Business Jet. Proceedings of the AIAA Guidance, Navigation, and Control Conference, 2019.

[4] G. Cooper and R. Harper. Handling Qualities and Pilot Evaluation. Journal of Guidance, Control, and Dynamics, 9(5):515-529, 1986. ISSN 0731-5090. doi: 10.2514/3.20142.

[5] R. C. Nelson. Flight Stability and Automatic Control. McGraw-Hill Education, 1998. ISBN 0-07-046218-6.

[6] A. E. Bryson and Y. C. Ho. Applied Optimal Control. Hemisphere, Washington D.C., 1975.

[7] K. P. Germann. T-6A Texan II In-Flight Simulation and Variable Stability System Design. Mississippi State University, 2006.

[8] E. S. Armstrong. A Design System for Linear Multivariable Control. New York: Marcel Dekker, 1980.

[9] E. Kreindler and D. Rothschild. Model-Following in Linear-Quadratic Optimization. American Institute of Aeronautics and Astronautics Journal, 14, no. 7:835-842, 1976.

[10] M. J. O’Brien and J. R. Broussard. "Feedforward Control to Track the Output of a Forced Model." Decision and Control including the 17th Symposium on Adaptive Processes. IEEE Conference on. IEEE, pages 835842, 1979.

[11] Brian L. Stevens, Frank L. Lewis, and Eric N. Johnson. Aircraft Control and Simulation: Dynamics, Controls Design, and Autonomous Systems. John Wiley \& Sons, 3 edition, 2015. ISBN 9781119174882 . doi: 10.1002/9781119174882. URL http://doi.wiley.com/10.1002/9781119174882.

[12] G. F. Franklin, J. D. Powell, and A. Emami-Naeini. Feedback Control of Dynamic Systems. Prentice Hall, 4th edition, 2002. ISBN 0130323934.

[13] J. J. E. Slotine and W. Li. Applied Nonlinear Control. Englewood Cliffs, N.J.: Prentice Hall, 1991.

[14] Standard. MIL-STD-1797A Requirements for Flying Qualities of Piloted Aircraft. Department of Defense, 1990.

[15] J. Ko, H. Lee, and J. Lee. In Flight Simulation for Flight Control Law Evaluation of Fly-by-Wire Aircraft. Agency for Defense Development, Daejeon, Republic of Korea, 2004.

[16] J. Ko and S. Park. Design of a Variable Stability Flight Control System. KSAS International Journal, 9(1), 2008.

[17] J. Ko and S. Park. Variable stability system control law development for in-flight simulation of pitch/roll/yaw rate and normal load. International Journal of Aeronautical and Space Sciences, 15(4): 412-418, 2014. ISSN 20932480. doi: 10.5139/IJASS.2014.15.4.412.

[18] C. Miller. Nonlinear Dynamic Inversion Baseline Control Law: Architecture and Performance Predictions. In AIAA Guidance, Navigation, and Control Conference, Guidance, Navigation, and Control and Co-located Conferences. American Institute of Aeronautics and Astronautics, aug 2011. doi: 10.2514/6.2011-6467. URL https://doi.org/10.2514/6.2011-6467. 
[19] P. Smith. A Simplified Approach to Non-linear Dynamic Inversion Based Flight Control. 23rd Atmospheric Flight Mechanics Conference, 1998. doi: 10.2514/6.1998-4461. URL http://arc.aiaa.org/ doi/10.2514/6.1998-4461.

[20] B. Bacon and A. Ostroff. Reconfigurable Flight Control using Non-linear Dynamic Inversion with a special Accelerometer Implementation. AIAA Guidance, Navigation, and Control Conference and Exhibit, 2000. doi: 10.2514/6.2000-4565. URL http://arc . aiaa.org/doi/10.2514/6.2000-4565.

[21] T. Cox and M Cotting. A Generic Inner-Loop Control Law Structure for Six-Degree-of-Freedom Conceptual Aircraft Design. In 43rd AIAA Aerospace Sciences Meeting and Exhibit, Aerospace Sciences Meetings. American Institute of Aeronautics and Astronautics, jan 2005. doi: 10.2514/6.2005-31. URL https://doi.org/10.2514/6.2005-31.

[22] S. Sieberling, Q. P. Chu, and J. A. Mulder. Robust Flight Control Using Incremental Nonlinear Dynamic Inversion and Angular Acceleration Prediction. Journal of Guidance, Control, and Dynamics, 33(6):17321742, 2010. ISSN 0731-5090. doi: 10.2514/1.49978. URL http://arc.aiaa.org/doi/10.2514/1. 49978.

[23] P. Simplício, M. D. Pavel, E. van Kampen, and Q. P. Chu. An Acceleration Measurements-Based Approach for Helicopter Non-linear Flight Control using Incremental Non-linear Dynamic Inversion. Control Engineering Practice, 21(8):1065-1077, 2013. ISSN 09670661.

[24] E. J. J. Smeur, Q. P. Chu, and G. C. H. E. de Croon. Adaptive Incremental Nonlinear Dynamic Inversion for Attitude Control of Micro Air Vehicles. Journal of Guidance, Control, and Dynamics, 39(3):450-461, 2016. ISSN 0731-5090. doi: 10.2514/1.G001490.

[25] E. J. J. Smeur, G. C. H. E. de Croon, and Q. P. Chu. Cascaded Incremental Nonlinear Dynamic Inversion Control for MAV Disturbance Rejection. Control Engineering Practice, pages 1-12, 2017.

[26] W. Bihrle. A Handling Qualities Theory for Precise Flight Path Control. Technical report, Air Force Flight Dynamics Laboratory, New York, 1966.

[27] M. H. Sandraey. Aircraft Design, A Systems Engineering Approach. John Wiley \& Sons, 2013.

[28] D. E. Bischoff. The Control Anticipation Parameter for Augmented Aircraft. Technical report, Naval Air Development Center, Warminster, 1981.

[29] D. A. Di Franco. In-flight Investigation of the Effects of Higher-Order Control System Dynamics on Longitudinal Handling Qualities. Technical report, Cornel Aeronautic Lab, Buffalo NY, 1968.

[30] J. Hodgkinson, W. J. LaManna, and J. L. Heyde. Handling qualities of aircraft with stability and control augmentation systems - a fundamental approach. 80:75-81, 021976.

[31] E. A. Morelli. Identification of Low Order System Models From Flight Equivalent Test Data. NASA Langley Research Center, 2000.

[32] D. J. Wilson, D. R. Riley, and K. D. Citurs. Aircraft Maneuvers for the Evaluation of Flying Qualitties and Agility, Volume2: Maneuver Descriptions and Selection Guide. Technical report, McDonnell Douglas Aerospace, St. Louis, 1993.

[33] C. Borst. CITAST: Citation Analysis and Simulation Toolkit. Delft University of Technology, Delft, 2004.

[34] M. A. Van den Hoek, C. C. de Visser, and D. M. Pool. Identification of a Cessna Citation II Model Based on Flight Test Data. In Advances in Aerospace Guidance, Navigation and Control, pages 259-277, 2018. ISBN 978-3-319-65283-2.

[35] B. Lubbers. A Model of the Experimental Fly-By-Wire Flight Control System for the PH-LAB. Delft University of Technology (unpublished master thesis), 2009.

[36] F. Grondman, G. Looye, R. O. Kuchar, Q. P. Chu, and E. Van Kampen. Design and Flight Testing of Incremental Nonlinear Dynamic Inversion-based Control Laws for a Passenger Aircraft. In 2018 AIAA Guidance, Navigation, and Control Conference, AIAA SciTech Forum. American Institute of Aeronautics and Astronautics, jan 2018. doi: 10.2514/6.2018-0385. URL https : //doi .org/10. 2514/6. 2018-0385. 
[37] E. N. Johnson and A. J. Calise. Pseudo-Control Hedging : a New Method for Adaptive Control, 2000.

[38] G. Looye, M. Thümmel, M. Kurze, M. Otter, and J. Bals. Non-linear Inverse Models for Control. Proceedings of the 4th Modelica Conference, pages 267-279, 2005.

[39] M. M. van Paassen, O. Stroosma, and J Delatour. DUECA - Data-driven Activation in Distributed RealTime Computation. In Modeling and Simulation Technologies Conference, Guidance, Navigation, and Control and Co-located Conferences. American Institute of Aeronautics and Astronautics, aug 2000. doi: 10.2514/6.2000-4503. URL https://doi.org/10.2514/6.2000-4503.

[40] R. C. van 't Veld. Incremental Non-linear Dynamic Inversion Flight Control: Implementation on a CS-25 Certified Fixed-wing Aircraft. Delft University of Technology (unpublished master thesis), 2016.

[41] AHS-3000. Attitude Heading Reference System: Installation Manual. Rockwell Collins, 2005.

[42] Cessna Aircraft Company. Cessna Citation II Maintenance Manual. Cessna Aircraft Company, 1987. 